X Whas a

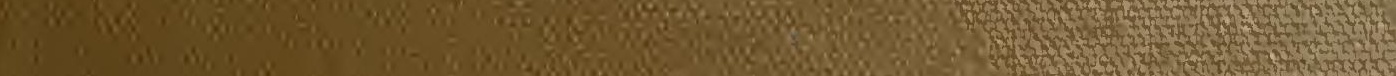

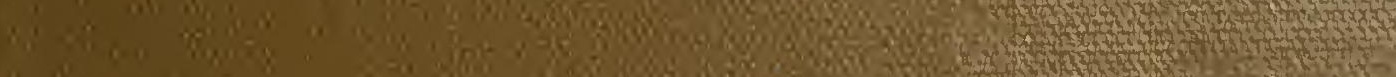

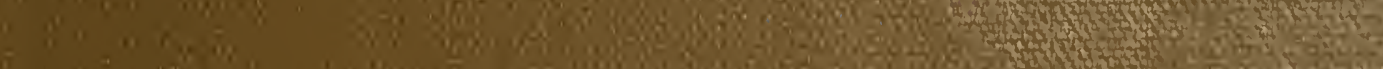
40"

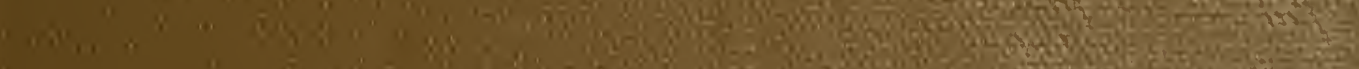

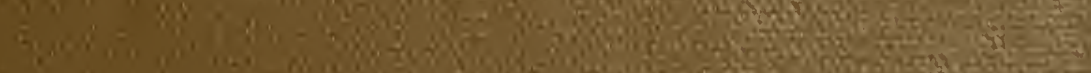

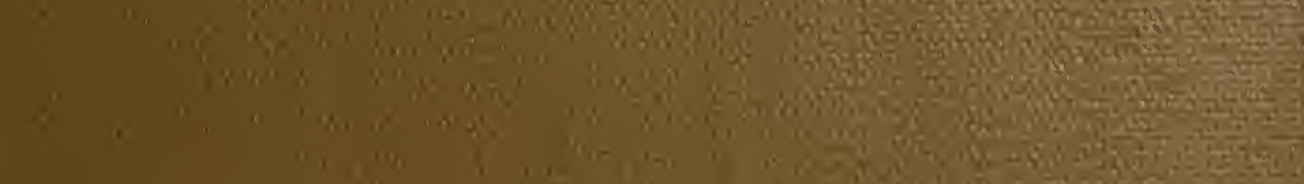

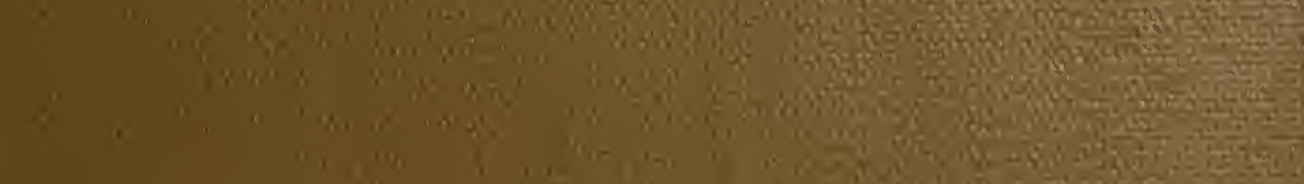

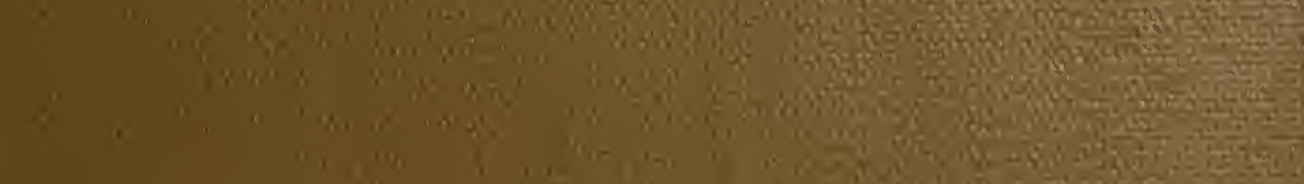

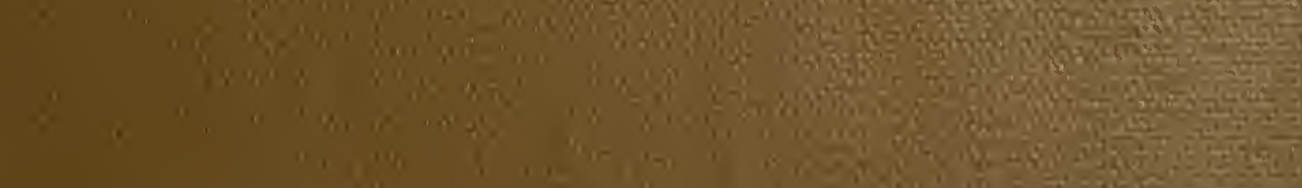

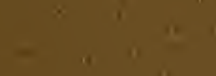

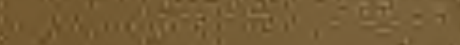

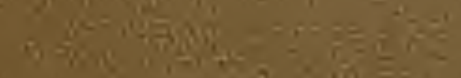

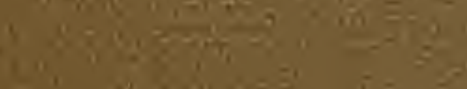

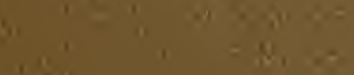
$\therefore \pi=3$

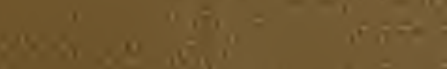
$+1$ in

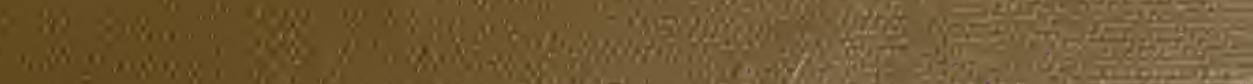

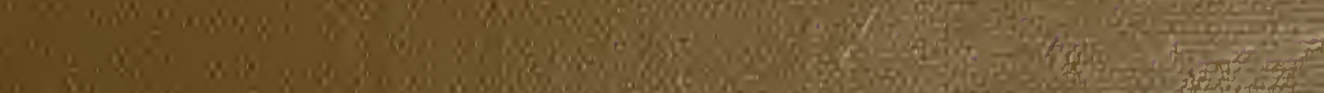

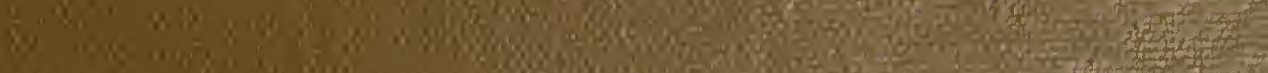

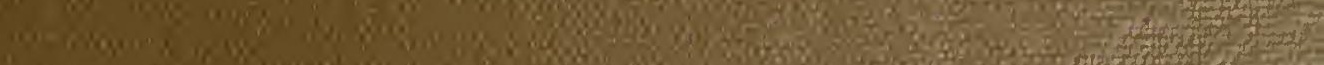

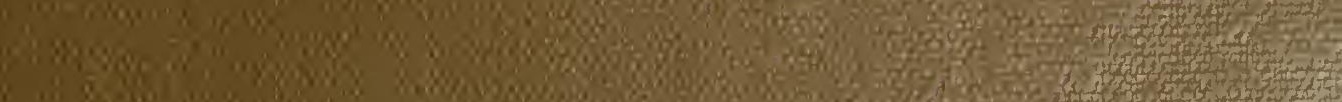

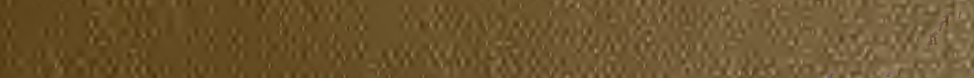

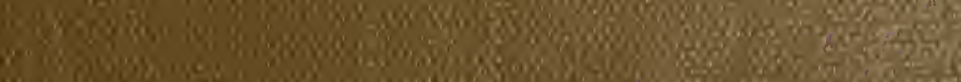




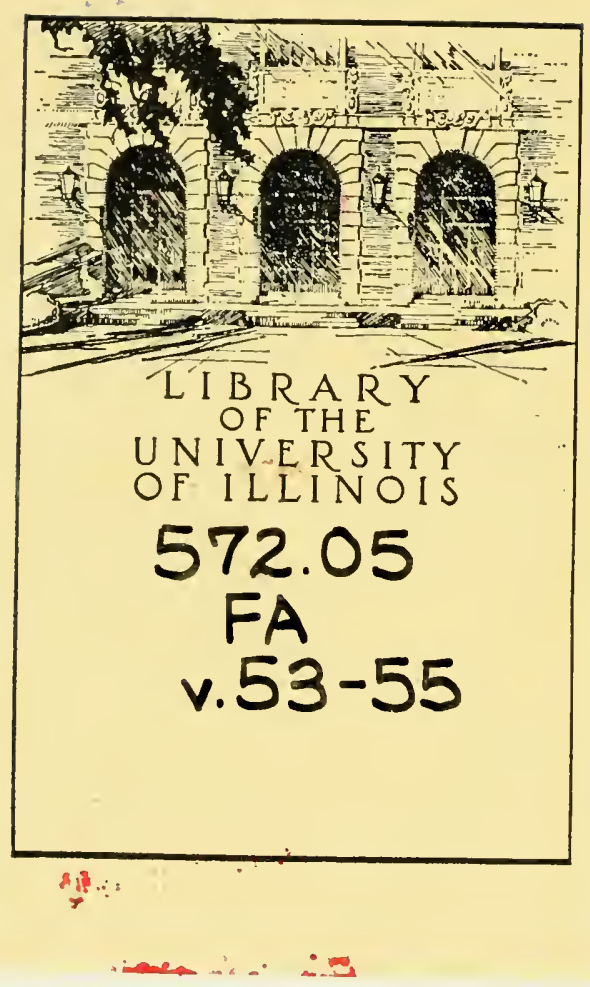




\section{UNIVERSITY LIBRARY UNIVERSITY OF ILLINOIS AT URBANA-CHAMPAIGN}

The person charging this material is responsible for its renewal or return to the library on or before the due date. The minimum fee for a lost item is $\$ 125.00, \$ 300.00$ for bound journals.

Theft, mutilation, and underlining of books are reasons for disciplinary action and may result in dismissal from the University. Please note: self-stick notes may result in torn pages and lift some inks.

Renew via the Telephone Center at 217-333-8400, 846-262-1510 (toll-free) or circlib@uiuc.edu.

Renew online by choosing the My Account option at: http://www.library.uiuc.edu/catalog/

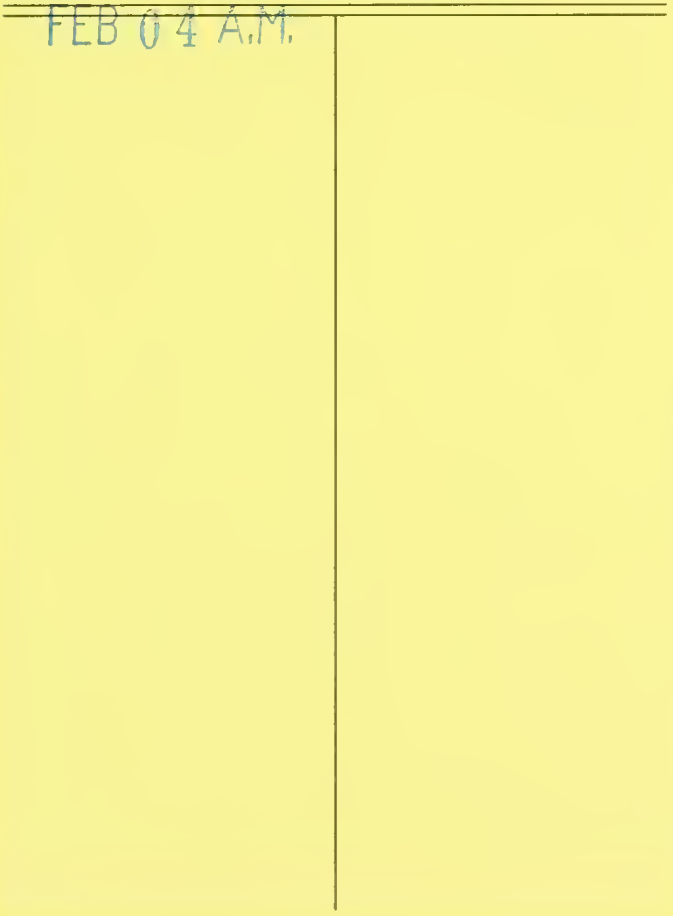







\title{
CHAPTERS IN THE PREHISTORY OF EASTERN ARIZONA, I
}

\author{
PAUL S. MARTIN \\ JOHN B. RINALDO \\ WILLIAM A. LONGACRE \\ CONSTANCE GRONIN \\ LESLIE G. FREEMAN, JR. \\ JAMES SCHOENWETTER
}

\author{
FIELDIANA : ANTHROPOLOGY \\ VOLUME 53 \\ Published by \\ CHICAGO NATURAL HISTORY MIUSEUM \\ SEPTEMBER 19, 1962
}







\title{
FIELDIANA: ANTHROPOLOGY
}

\author{
A Continuation of the
}

ANTHROPOLOGICAL SERIES

of

FIELD MUSEUM OF NATURAL HISTORY

VOLUME 53

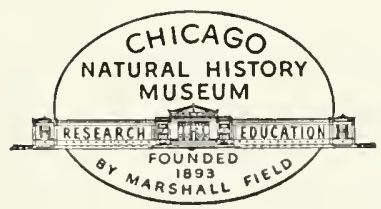

CHICAGO NATURAL HISTORY MUSEUM

CHICAGO, U.S.A.

1962 

CHAPTERS IN THE PREHISTORY OF EASTERN ARIZONA, I 


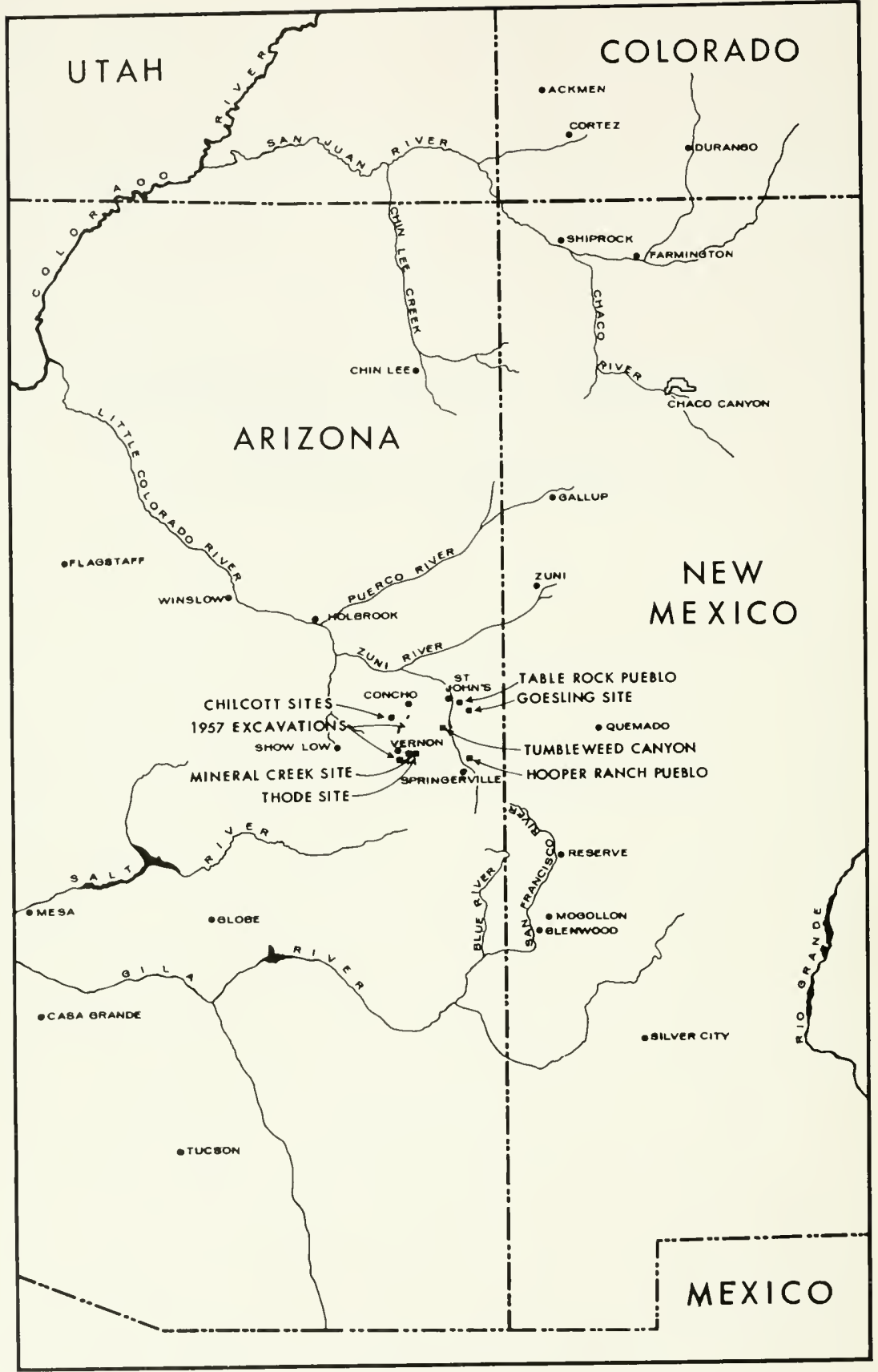

MAP SHOWING EASTERN ARIZONA AND WESTERN NEW MEXICO 


\title{
CHAPTERS IN THE PREHISTORY OF EASTERN ARIZONA, I
}

\author{
PALL S. MARTIN \\ JOHN B. RINALDO \\ WILLIAM A. LONGACRE \\ CONSTANCE CRONIN \\ LESLIE G. FREEMAN. JR. \\ JAMES SCHOENINETTER
}

\author{
FIELDIANA: ANTHROPOLOGY \\ VOLUME 53 \\ Published by \\ CHICAGO NATURAL HISTORY MUSEUM \\ SEPTEMBER 19, 1962
}


Edited by. Lillian A. Ross

Library of Congress Catalog Card Number: 62-21153

PRINTED IN THE UNITED STATES OF AMERICA BY CHICAGO NATURAL HISTORY MUSEUM PRESS 


\section{Preface}

\section{Field Season of 1960}

The field research of the 1960 season was remarkably interesting. Several projects were undertaken, the results of which are described in this report.

The National Science Foundation extended financial aid (Grant No. G-13039) to the expedition and this permitted us to carry on an archaeological reconnaissance; to cmbark on a palaeo-ecological inquiry by means of pollen analysis; and to dig a pre-ceramic site (Tumblcweed Canyon Site) of pithouses. These ventures could not have been undertaken without this aid.

In addition, with Museum funds, five other sites werc cxcavated, making, with the pre-pottery village, a total of six. These are (in alphabetical order):

1. Chilcott Sites (3), near Mesa Redondo and about seven miles southwest of Concho, Arizona (Sec. 5, Twp. 11 N., R. 25 E., G. and S.R.M.).

2. Goesling Site, located about two miles east of St. Johns, Arizona, and overlooking the valley of the Little Colorado River (NE 1/4, Sec. 2, Twp. 12 N., R. 28 E., G. and S.R.M.).

3. The Great Kiva, Hooper Ranch Pueblo (see Martin, Rinaldo and Longacre, 1961), near Springerville, Arizona, on the banks of the present channel of the Little Colorado River (NE 1/4, SW 1/4, Sec. 8, Twp. 9 N., R. 29 E., G. and S.R.M.).

4. Rim Valley Pueblo, on the Hooper Ranch, situated high up on the edge of a mesa overlooking the valley of the Little Colorado River and the Hooper Ranch Pueblo (SE 1/4, NE 1/4, Scc. 18, Twp. 9 N., R. 29 E., G. and S.R.M.).

5. Thode Site, on the west bank of the east fork of Mineral Creek, near Highway 60 (SW 1/4, NE 1/4, Scc. 13, Twp. 10 N., R. 25 E., G. and S.R.M.).

6. Tumbleweed Canyon Site, on the west bank of the Little Colorado River, about halfway between St. Johns and Springerville, Arizona, far from a highway; high up on an "island" mesa of lava, overlooking 
Lyman Dam Reservoir (NE 1/4, SE 1/4, Sec. 17, Twp. 11 N., R. 28 E., G. and S.R.M.). Financial aid supplied by funds from the National Science Foundation.

The sites are on ranches owned by:

1. Mr. D. Chilcott, managed by Mr. Frank Stradling, Concho, Arizona.

2. Mr. Al. H. Goesling, St. Johns, Arizona.

3 and 4. Mr. Rob Hooper, Springerville, Arizona.

5. Mr. Earl Thode, Vernon, Arizona.

6. Mr. Pacer Wiltbank, Eagar, Arizona.

I have listed these public-spirited gentlemen separately so that their names will stand forth prominently and everyone will recognize their contribution to archaeological research. I hope more will follow their example. It is a pleasure to record here the thanks of the Museum and of the members of the expedition and to state that our goals have been greatly advanced by their unselfish help. We were permitted to dig without hindrance and to bring back to the Museum, for research and exhibition purposes, all of the specimens recovered. Many thousands of people will benefit directly and indirectly from this arrangement and will derive educational and cultural stimulation as well as satisfaction of a common curiosity about man's past.

In addition. I want especially to thank the members of the expedition for the aid that they rendered in digging, in processing and cataloging artifacts, in mending and restoring pottery, in housekeeping and in maintaining an enviable record of spontaneity, of good will and cooperation, of cheerfulness, of harmony, and of zest for all phases of the work, whether glamorous or dull: Mr. William Alschuler, Miss Ellen Chase, Mr. David Herod, Mr. Gardner Lane, Mr. William A. Longacre, Mrs. Martha Perry, Mr. Pat Romane, Mrs. John B. Rinaldo, Mr. John Saul, Mr. Roland Strassburger, and Mr. John Wells. Assisting also were several neighbors and helpers of other seasons: Mr. Wilfred Barreras, Mr. Joe Goodman, Mr. Genaro Nuarez, Mr. Gilbert Padilla and Mr. Kenneth Penrod.

The palynological project was a new venture for us and was financed entirely by the grant from the National Science Foundation. Mr. James Schoenwetter was in charge of this project and has written an excellent report that appears in this volume. I find it suggestive and informative. Without the advice of and the frequent consultations with Terah L. Smiley and Paul S. Martin of the Geochronology Laboratories of the University of Arizona, Schoenwetter's objectives could not have been attained. 
The archaeological reconnaissance, made possible by a grant from the National Science Foundation, was undertaken by Mr. Longacre. It was successfully accomplished because of Mr. Longacre's skill in establishing cordial rapport with neighboring ranchers, some of whom were hostile due to past actions of prospectors. In the preliminary work, Mr. Longacre was given admirable assistance by Mr. Leigh Richey of St. Johns, Arizona.

I also take pleasure in thanking for their assistance: Mrs. Elizabeth Brawley, St. Johns; Mr. and Mrs. Wayne Brinkerhoff, Snowflake; Mr. and Mrs. J. R. Carter, Snowflake: Mr. Vernon Frazier, Snowflake; Mr. and Mrs. Jake Kittle, Show Low: Mr. and Mrs. John D. Leverton, Concho: Mrs. Leola Mineer, St. Johns; Mr. Verl Rhoton, Lakeside; Mrs. Merle Thomas. Concho; Mr. and Mrs. Harvey Wilhelm, Snowflake; Mr. and Mrs. Ira Willis and Mr. Kelley Willis, Snowflake; Mr. Ozie Wilson, Pinetop; and Mr. and Mrs. Richard Wilson, Pinetop.

Our work is slowly becoming known in the Vernon area and more and more people are beginning to respect archaeology as opposed to pot-hunting. Last summer we held an open house one Sunday afternoon to show our friends how we wash and classify pottery; how we restore smashed and incomplete pottery (and demonstrate that we recover mostly sherds and broken pots-rarely a whole one); what we recover in the way of artifacts and how we catalogue them: how we record architectural details; and what we "do" with these data. More than a hundred people came, in spite of one of the heaviest rainfalls of the summer.

Analyzing and preparing our data for publication are large tasks that have to be wedged into a crowded Museum schedule. Realizing that I alone could not do full justice to the analysis of the pottery that we recovered, I enlisted the help of Mr. Walter Boyer, sometime artist in the Department of Anthropology, and of two advanced studentassistants from the Department of Anthropology of the University of Chicago, Miss Constance Cronin and Mr. Leslic Freeman.

The possible origin of a pottery type called Snowflake Black-onWhite-as yet really not too well known and not described has been examined by Miss Cronin and Mr. Boyer. The examination pursued two trails: one admittedly subjective: and the other (hopefully) "oljective." I placed approximately 2500 black-on-white painted (decorated) sherds from five sites at the disposal of Miss Cronin. These sherds represented several pottery types ranging in time from about A.D. 750 to about 1200 . Miss Cronin (assisted in the preliminary stages of the study by $\mathrm{Mr}$. Boyer) grouped the sherds into lots bearing identical or similar elements 
of designs, but without regard to type, site, or chronology; for example, all sherds bearing squiggly hatch, or ticked lines or pendent triangles were put into separate piles and then counted. Then she re-sorted the same sherds by site, by type, and by chronology and then separated these groups into lots bearing similar or identical elements of design. These were counted, percentages derived, and graphs drawn. Thus the study was "quantified." Miss Cronin's report is included in this volume.

The following civic-minded and generous persons have thought well enough of our work to contribute financially to the expedition: Mr. C. E. Gurley, Gallup, New Mexico; Mr. and Mrs. Maxwell Hahn, Scarsdale, New York; Dr. Charles W. Keney, Gallup, New Mexico; and Mr. Judd Sackhein, Chicago. The gifts of these thoughtful people reach far beyond the materialistic side of things; they reach into our hearts and cheer us. I hope the results of the expedition, embodied in this report, will bring them pleasure.

Our immediate neighbors in Vernon continued to help us in manifold ways and to be enthusiastic about our work. I am happy to thank Mr. and Mrs. Tom Cox, Mr. and Mrs. Donald Goodman, Mr. and Mrs. Charles Gillespie, Mr. and Mrs. Leon Gillespie, Mr. and Mrs. Milton Gillespie, Mr. and Mrs. Cecil Naegle, Mr. and Mrs. Leonard Penrod, Kenneth Penrod, Mr. and Mrs. Floyd Penrod, Mr. and Mrs. Claude Phipps, and Mr. and Mrs. Eben Whiting for all favors, large and small.

Miss Lillian Ross, Associate Editor of Scientific Publications, has earned our gratitude for help in seeing this report through the press and for catching errors of omission and commission.

Mr. Bertram J. Woodland, Associate Curator, Petrology, identified the materials from which the stone artifacts were made; Dr. Albert Forslev, sometime Associate Curator, Mineralogy, and now on the staff of the College of William and Mary in Norfolk, Virginia, made mineralogical analyses of two samples of clay; and Dr. Fritz Haas, Curator Emeritus, Lower Invertebrates, checked the shell specimens. We are grateful to these gentlemen for their help.

Dr. Frederick J. Dockstader, Dr. Fred Eggan, Mr. Byron Harvey III, and Mrs. Marjorie F. Lambert were kind enough to examine photographs and data of our sacred stone image and to aid us in our attempts to identify it. We appreciate their assistance.

Mrs. Agnes McNary Fennell, my secretary, and Miss Lillian Novak typed the manuscript and tables and deserve great thanks for their work. Mrs. Fennell also made the index.

Again it is my pleasure and privilege to thank the administration for its support of the Vernon project. President Stanley Field, Dr. Clifford C. 
Gregg, Director, and our Board of Trustees once more provided us with funds for the expedition. I hope they will derive as much pleasure and satisfaction from the results of our work as I do in expressing my appreciation and indebtedness to them for their sustained interest and assistance.

January 1, 1961

PaUl S. Martin 



\section{Contents}

List of Illustrations ............. 15

I. Architectural Detalls . . . . . . . . . . . 19

Tumbleweed Canyon Site . . . . . . . . . . . . . . 19

House A . . . . . . . . . . . . . 19

Shape . . . . . . . . . . . . . 19

Walls ................... . . 19

Floor . . . . . . . . . . . . 19

Firepit . . . . . . . . . . . . . 19

Pit . . . . . . . . . . . 19

Postholes . . . . . . . . . . . . . . 20

Roof . . . . . . . . . . . . . 20

Milling Area . . . . . . . . . . . . . 20

General Comments . . . . . . . . . . . . 20

House B . . . . . . . . . . . . . . . . . . . . . . . 20

Shape . . . . . . . . . . . 20

Walls ..................... 20

Floor . . . . . . . . . . . . . . 22

Firepit . . . . . . . . . . . . 22

Pits . . . . . . . . . . . . . . . 22

Postholes . . . . . . . . . . . . . . . 22

Roof . . . . . . . . . . . . . 22

General Comments . . . . . . . . . . . . 22

House D . . . . . . . . . . . . . 22

Shape . . . . . . . . . . . . 22

Walls . . . . . . . . . . . . . 23

Floor . . . . . . . . . . . 23

Firepit . . . . . . . . . . . . 25

Postholes . . . . . . . . . . . . . . 25

Roof . . . . . . . . . . . 25

Milling Area . . . . . . . . . . . . . . 25

General Comments . . . . . . . . . . 25

Storage Pits(?) . . . . . . . . . . . . 25

Goesling Site . . . . . . . . . . . . 26

Shape . . . . . . . . . . . . 26 26

Walls ................... 26

Floors . . . . . . . . . . . . . 2 28

Firepits . . . . . . . . . . . . . . . . . . 28

Entrance . . . . . . . . . . . . . 28

Pit . . . . . . . . . . . . . . 29

Postholes . . . . . . . . . . . . . . . 29 
Roof

General Comments

Chilcott Sites

Chilcott Site 1

Number of Rooms

Dimensions of Rooms

Walls

Floors

Firepits . . . . . . . . . . . . . 34

Postholes . . . . . . . . . . . . . . . 34

Ventilator . . . . . . . . . . . . 36

Roof . . . . . . . . . . . . . 37

General Comments . . . . . . . . . . . . . 37

Chilcott Sites 2 and 3 . . . . . . . . . . . 37

Number of Structures . . . . . . . . . . . . . . . 37

Walls.............. . . . . 37

Floors . . . . . . . . . . . . . 37

Firepit . . . . . . . . . . . . . . . 37

Postholes . . . . . . . . . . . . . . 37

Roof . . . . . . . . . . . . . . . 37

Shape . . . . . . . . . . . . . . . 37

Thode Site . . . . . . . . . . . . . . . . . 40

Number of Rooms . . . . . . . . . . . . . . . . 40

Walls . . . . . . . . . . . . . . . 40

Floor . . . . . . . . . . . . . . . . 40

Firepit . . . . . . . . . . . . . . 40

Entrances . . . . . . . . . . . . . 4 40

Pits . . . . . . . . . . . . . . . 40

Postholes . . . . . . . . . . . . . . . . 40

Roof . . . . . . . . . . . . . 40

General Comments . . . . . . . . . . . . 40

Rim Valley Pueblo . . . . . . . . . . . . . . . . 40

Arrangement of Pueblo Parts . . . . . . . . . . . . . . 43

Number of Rooms . . . . . . . . . . . . . . . . . . . 43

Dimensions of Rooms . . . . . . . . . . . . . . . 43

Walls... . . . . . . . . . . . . . 43

Ventilators and Niches . . . . . . . . . . . . . . 46

Floors . . . . . . . . . . . . 48

Firepits . . . . . . . . . . . . . . . 48

Ladder pits(?) . . . . . . . . . . . . . . . . . . . 48

Vault . . . . . . . . . . . . . . 48

Bin . . . . . . . . . . . . . 48

Ceiling . . . . . . . . . . . . . . . 50

General Comments . . . . . . . . . . . . 50

The Great Kiva, Hooper Ranch Pueblo . . . . . . . . . . . . . 53

Shape . . . . . . . . . . . . . . . . 53

Dimensions . . . . . . . . . . . . . . . 53

Walls . . . . . . . . . . . . . 53

Pictographs . . . . . . . . . . . . . 54 
Niche . . . . . . . . . . . . . 54

Recessed Posts . . . . . . . . . . . . . . . . . 54

Floor . . . . . . . . . . . . 54

Bin ....................... 55

Firepit . . . . . . . . . . . . . 55

Deflector . . . . . . . . . . . . 57

Vaults... . . . . . . . . . . 57

Crypt . . . . . . . . . . . . . 57

Pits . . . . . . . . . . . . . . 58

Ramp Entryway . . . . . . . . . . . . . . . 58

Posts and Postholes . . . . . . . . . . . . . . 59

Roof . . . . . . . . . . . . . . . 59

Comparisons . . . . . . . . . . . . . . . 60

Summary of Secular Architecture . . . . . . . . . . . 60

II. Some Convergences and Continuties . . . . . . . . . . . 64

The Great Kiva . . . . . . . . . . . . . . . . . 64

The Sacred Stone Image . . . . . . . . . . . . . 69

III. Pottery. . . . . . . . . . . . . . . . . . 75

General Remarks . . . . . . . . . . . . . . 75

Whole or Restorable Pots Recovered . . . . . . . . . . . . 78

Relative Popularity of Several of the Significant Painted Pottery Types . 80

IV. Statistical Analysis of Painted Pottery Types from Upper Little Colorado Dralnage . . . . . . . . . . . . . . . . 87

Introduction . . . . . . . . . . . . . . . . . . . 87

Choice of Materials . . . . . . . . . . . . . . . . . . . . 88

Some Methodological Considerations . . . . . . . . . . . . . . . 88

Basic Procedure . . . . . . . . . . . . . . . . . . . . . . 90

Inter-Site Seriation . . . . . . . . . . . . . . 93

Interpretation . . . . . . . . . . . . . . 93

Intra-Site Seriations . . . . . . . . . . . . . . 94

Goesling Site . . . . . . . . . . . . . . . 94

Rhoton and Thode Sites . . . . . . . . . . . . 94

Chilcott Site . . . . . . . . . . . . . . . . 95

Rim Valley Pueblo . . . . . . . . . . . . . . . 98

Hooper Ranch Pueblo . . . . . . . . . . . . . . . . . 99

Conclusions . . . . . . . . . . . . 102

V. Analysis of Pottery Design Elements . . . . . . . . . . . 105

Sorting . . . . . . . . . . . 106

Analysis . . . . . . . . . . . . . . 107

Conclusions. . . . . . . . . . . . . 107

Kiatuthlanna Black-on-White . . . . . . . . . . . . . 109

Red Mesa Black-on-White . . . . . . . . . . 109

Snowflake Black-on-White . . . . . . . . . . . 110

VI. ARtifacts . . . . . . . . . . . . 115

Introduction . . . . . . . . . . . . . . . 115

Tools Used in Preparation and Storage of Food . . . . . . . . . . 115

Manos. . . . . . . . . . . . . . 116

Rubbing Stones . . . . . . . . . . . 119 
Pestles

Metates

Small Metate-like Grinding Stones

Mortars

Pot Covers

124

. . 125

Hammerstones . . . . . . . . . . . . . 126

Pot Rests . . . . . . . . . . . . . . . 126

Tools Used in Pottery-Making . . . . . . . . . . . . . . 126

Tools Used in Construction of Houses . . . . . . . . . . . . . . 128

Axe . . . . . . . . . . . . . . 129

Maul . . . . . . . . . . . . . . . 129

Axe-Grinding Slabs . . . . . . . . . . . . . . . . 129

Choppers . . . . . . . . . . . . . . 130

Tools and Weapons Used in Hunting and Warfare . . . . . . . . . 130

Projectile Points . . . . . . . . . . . . . . . . . 130

Arrow-Shaft Tools . . . . . . . . . . . . . . . . . . 132

Household Utility Tools . . . . . . . . . . . . . . . . . 135

Flake Knives . . . . . . . . . . . . . . . . . . . . . 135

Scrapers . . . . . . . . . . . . . . . 136

Saws . . . . . . . . . . . . . 136

Drills . . . . . . . . . . . . . . . . 139

Weaving Tools . . . . . . . . . . . . . . . 139

Bone Awls . . . . . . . . . . . . . . . . . . . 139

Spindle Whorls . . . . . . . . . . . . . . . . 140

Ornaments . . . . . . . . . . . . . . 140

Pendants . . . . . . . . . . . . . . . . 143

Beads . . . . . . . . . . . . . . . 144

Bracelets . . . . . . . . . . . . . . 144

Rings . . . . . . . . . . . . . . 144

Bone Ring Material . . . . . . . . . . . . . 144

Tinkler . . . . . . . . . . . . . 145

Cut Shell . . . . . . . . . . . . . . . 145

Ceremonial Objects . . . . . . . . . . . . . . . 145

Cylinder Stones . . . . . . . . . . . . . . 145

Sacred Stone Image . . . . . . . . . . . . . . . 146

Worked Sherds . . . . . . . . . . . . . . 146

Summary . . . . . . . . . . . . . . . 146

ViI. Archaeological Regonnaissance in Eastern Arizona . . . . . . 148

Introduction . . . . . . . . . . . . . . . . . . . . . . . 148

Organization . . . . . . . . . . . . . . 150

Field Procedure . . . . . . . . . . . . . . . . . 151

Temporal-Spatial Occupation of Surveyed Region . . . . . . . . . 151

Site Locations . . . . . . . . . . . . . . . . . . . 152

General Summary . . . . . . . . . . . . . 155

Conclusions .. . . . . . . . . . . . . . 163

ViII. Pollen Analy'sis of Eighteen Archaeologigal Sites in Arizona and

New Mexico . . . . . . . . . . . . . . . . . . . . . . . 168

Acknowledgments . . . . . . . . . . . . . . . . . . . 168

Introduction . . . . . . . . . . . . . . . . 168 
Methods and Techniques . . . . . . . . . . . 170

Sampling . . . . . . . . . . . 170

Extraction . . . . . . . . . . . . 171

Analysis . . . . . . . . . . . . 172

Common Names of Pollen Types and Ecological Notes . . . . . . . 173

Results . . . . . . . . . . . . . . . 177

Arroyo Sites . . . . . . . . . . . . . . 177

Beach Sites . . . . . . . . . . . . . 178

Pithouse Village Sites . . . . . . . . . . . . . . . . 178

Pueblo Sites . . . . . . . . . . . . . . . . 181

Surface Samples . . . . . . . . . . . . 185

Conclusions . . . . . . . . . . . . . 187

Interpretations and Inferences . . . . . . . . . . . . 191

Climatic Change . . . . . . . . . . . . . . 191

The Nature of Pre-Existing Environmental Conditions . . . . . . 194

Relationship of Prehistoric Environments to Prehistory . . . . . . 198

Appendix 1: Sample Collection Technique . . . . . . . . . . 206

Appendix B: Pollen Extraction Technique . . . . . . . . . . 207

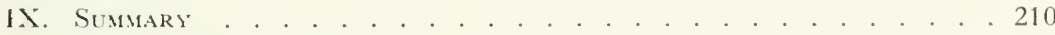

Tumblewecd Canyon Site . . . . . . . . . . . . . 210

Goesling Site . . . . . . . . . . . . . . . 212

Chilcott Sites . . . . . . . . . . . . . 212

Thode Site . . . . . . . . . . . . . 212

Rim Valley Pueblo . . . . . . . . . . . . . . . 213

Great Kiva, Hooper Ranch Pueblo . . . . . . . . . . . . 213

Settlement Patterns . . . . . . . . . . . . . . . 215

Tumbleweed Canyon Site . . . . . . . . . . . . . 215

Gocsling Site . . . . . . . . . . . 216

Chilcott Sites . . . . . . . . . . 2 216

Thode Site . . . . . . . . . . . 217

Rim Valley Pueblo . . . . . . . . . . . . 218

Great Kiva, Hooper Ranch Pucblo . . . . . . . . . . 220

Analysis of Elements of Pottery Design . . . . . . . . . . . . 223

Archaeological Reconnaissance . . . . . . . . . . . . . . . 224

Pollen Analysis . . . . . . . . . . . . . . . . . . . . . . . . 225

BibliograpiYY . . . . . . . . . . . . . . . . 228

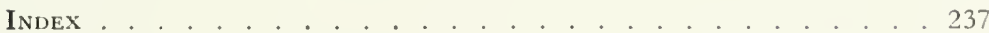





\section{List of Illustrations}

\section{Text Figures}

PAGE

1. Panoramic view of Tumbleweed Canyon Site and mesa from across the canyon 20

2. Sketch map of Tumbleweed Canyon Site and environs . . . . . . . . . 21

3. House A, Tumbleweed Canyon Site, showing manos, broken metates, and roof beam fragments . . . . . . . . . . . . . . . . . . 22

4. Plans and sections of houses and pits, Tumbleweed Canyon Site . . . . . 23

5. Milling area, House A, Tumbleweed Canyon Site, showing manos and broken metates . . . . . . . . . . . . . 24

6. House B, 'Tumbleweed Canyon Site, showing curb around edge of house and firepit

7. House D, Tumbleweed Canyon Site, showing broken metate fragments near center of house and rocks piled up as walls around edge of house

8. Pithouse A, Goesling Site, showing postholes, firepit in center and southern recess in background . . . . . . . . . . . . . 26

9. Plans and sections of Pithouses A and B, Goesling Site . . . . . . . . 27

10. Veneer masonry which reinforced north wall of Pithouse A, Goesling Site . . 28

11 Pithouse B, Goesling Site, showing shallow pit in northeast corner and quadrangular arrangement of postholes

12. Rooms 1 and 5 in foreground and Structure 2 in background, Chilcott Site 1 , showing alignment of postholes in rooms, and relationship of rooms with masonry walls to larger structure . . . . . . . . . . . 30

13. Plans and sections of Chilcott Site 1 . . . . . . . . . . . . . 31

14. Rooms 3 and 4, Chilcott Site 1, showing reduction in entryway . . . . . . 32

15. Detail of firepit, deflector, ventilator opening and damper slab, Room 4, Chilcott Site 1 . . . . . . . . . 33

16. View of Room 1, Chilcott Site 2, showing uneven floor and area of burned post and rocks near center of structure . . . . . . . . . . . . . . . 34

17. Plans and sections of Chilcott Site 2 (left) and Site 3 (right) . . . . . 35

18. Room 1, Chilcott Site 3, showing basalt boulder walls and general shape of structure

19. Room 2, Chilcott Site 3, showing rectangular frepit . . . . . . . 38

20. Thode Site . . . . . . . . . . . . . . . 38

21. Plan and sections of Thode Site . . . . . . . . . . . . . . 39

22. Rim Valley Pueblo, looking south . . . . . . . . . . . . . . 41

23. Plan and sections of Rim Valley Pueblo . . . . . . . . . . . . . . . 42

24. Outer wall of Room I, Rim Valley Pueblo . . . . . . . . . . . 43 
25. Rectangular doorway in north wall of Room G, Rim Valley Pueblo

26. Oval ventilator in south wall of Room C, Rim Valley Pueblo

27. Niche in north wall of Room A, Rim Valley Pueblo

28. Ring slab from Room C, Rim Valley Pueblo, possibly frame for vent

29. Flour receptacles and corner bin in southeast corner of Room B, Rim Valley Pueblo

30. Firepit, ladder-pit, ventilator, and damper slab, Room A, Rim Valley Pueblo

31. Room C, Rim Valley Pueblo, with Room B at left and Room H at right . .

32. Hooper Ranch Pueblo, showing Great Kiva in foreground and dwelling rooms in background

33. Plan and sections of Great Kiva and adjacent rooms at Hooper Ranch Pueblo

34. Great Kiva, Hooper Ranch Pueblo, from the west. Ramp entryway and deflector in background; postholes and vaults in foreground

35. Detail of masonry in face of bench on north side of Great Kiva, Hooper Ranch Pueblo

36. Detail of niche in face of bench on south side of Great Kiva, Hooper Ranch Pueblo

37. Deflector, Great Kiva, Hooper Ranch Pueblo, viewed from ramp entryway . 56

38. South vault, Great Kiva, Hooper Ranch Pueblo . . . . . . . . . . . . 56

39. Ramp entryway, Great Kiva, Hooper Ranch Pueblo, showing "vestibule" area and narrower portion beyond; deflector slab in foreground

40. View through ring slab cover of erypt, Great Kiva, Hooper Ranch Pueblo, showing stone image and miniature jar . . . . . . . . . . . . . .

41. Crypt in Great Kiva, Hooper Ranch Pueblo, with covers removed, showing construction detail of interior and objects in position . . . . . . . . .

42. Painted sacred stone image, Great Kiva, Hooper Ranch Pueblo . . . . . 70

43. Snowflake Black-on-White pottery . . . . . . . . . . . . . . . . 78

44. Brown indented corrugated pottery . . . . . . . . . . . . . . . . . 79

45. MeDonald Corrugated bowl . . . . . . . . . . . . . . . . . . . 79

46. Sehematic illustration of relative similarity between samples of pottery. (a) Chilcott Sites; (b) Rim Valley Pueblo; $(c)$ Hooper Ranch Pueblo; and (d) site totals

47. Percentages of three pottery types by levels at Goeslin Site $_{y}$. . . . . . 92

48. 'Trends in painted pottery types: samples from Chilcott Sites and Rim Valley Pueblo .

49. Trends in painted pottery types: samples from Hooper Ranch Pueblo . . . I01

50. One-hand manos, Tumbleweed Canyon Site . . . . . . . . . . . . . 117

51. Intermediate and late types of manos, Rim Valley Pueblo, Thode Site, Chilcott Site 1 . . . . . . . . . . . . . . . . . .

52. Rubbing stones, miscellaneous types, Goesling Site, Thode Site, Rim Valley Pueblo, Hooper Ranch Pueblo . . . . . . . . . . . . . . . 120

53. Pestles, miscellaneous types, and hammerstones, Tumbleweed Canyon Site, Rim Valley Pueblo . . . . . . . . . . . . . . . . 12

54. Metates: left speeimen basin type, center specimen with trough open at one end only, right specimen with through trough . . . . . . . . . . 123

55. Mortar, Thode Site . . . . . . . . . . . . . 125 
56. Maul, axe and pot cover, Rim V'alley Pueblo, Hooper Ranch Pueblo : . 127

57. Medicine cylinders and pot polishing stones, Hooper Ranch Pueblo, Rim Valley Pueblo, Goesling Site . . . . . . . . . . . . 128

58. Axe-grinaing slab, Hooper Ranch Pueblo . . . . . . . . . . . . . . . 131

59. Choppers and large scraper, Tumbleweed Canyon Site, Rim Valley Pueblo,

Chilcott Site 1, Goesling Site . . . . . . . . . . . 131

60. Projectile points, miscellaneous types . . . . . . . . . . . . . 133

61. Blades, Tumbleweed Canyon Site, Hooper Ranch Pueblo . . . . . . . . 134

62. Abrading stones, arrow-shaft tool and smooth saw, Hooper Ranch Pueblo . 135

63. Flake knives and small scrapers, Tumbleweed Canyon Site, Chileott Site 1, Goesling Site, Hooper Ranch Pueblo . . . . . . . . . . . . 137

64. Drills, punches, saws and blades, Hooper Ranch Pueblo, Goesling Site, Chilcott Site 1, Rim Valley Pueblo . . . . . . . . . . . . . . 138

65. Bone awls, incised bone fragment, bodkin tip and ring material, Goesling Site, Rim Valley Pueblo, Hooper Ranch Pueblo, Thode Site

66. Spindle whorls, worked sherds and miniature jar. Chilcott Site 1, Goesling Site. Hooper Ranch Pueblo

67. Pendants, bracelet fragments, and ring fragment, Goesling Site, Hooper Ranch Pueblo

68. Map showing area of archaeological survey, east-central Arizona . . . . 149

69. Projectile points from pre-pottery sites . . . . . . . . . . . . 158

70. Miscellaneous tools from pre-pottery sites . . . . . . . . . . . 158

71. Scrapers and utilized flakes from pre-pottery sites . . . . . . . . 159

72. Serapers from pre-pottery sites . . . . . . . . . . . . . . 160

-3. Large bifacially percussion-flaked scrapers/choppers from pre-pottery sites . 161

7. Choppers and manos from pre-pottery sites . . . . . . . . . . . . . . 162

75. Pollen diagrams of archaeological sites in Vernon, Arizona, area . facing page 168

76. Inalyses of three samples of pollen from modern surface and pollen diagrams of two archaeological sites in Pine Lawn, New Mexico, area . . facing page 172

77. Important palynological features of samples of pollen from occupation levels at archaeological sites in Vernon, Arizona, area . . . . . . . 175

-8. Samples of pollen from modern surface at various elevations in Vernon, Arizona, area.

\section{List of Tables}

1. Totals of sherds, Goesling Site . . . . . . . . . . . . . . 81

2. Totals of sherds, Chilcott Sites . . . . . . . . . . . . . . 82

3. Totals of sherds, Thode Site . . . . . . . . . . . . . . 83

4. Totals of sherds, Rim Valley Pueblo . . . . . . . . . . . . . . . . . 84

5. 'Totals of sherds, Great Kiva, Hooper Ranch Pueblo . . . . . . . . . 85, 94

6. Sample size of sherds and final matrix for inter-site seriation . . . . . . . 93

7. Sample size of sherds from Goesling Site . . . . . . . . . . . . 94

8. Sample size of sherds and final matrix, Chilcott Site . . . . . . . . 9)

9. Sample size of sherds and final matrix, Rim Valley Pueblo . . . . . . . 98 
10. Sample size of sherds and final matrix, Hooper Ranch Pueblo .

11. Percentages of given types by design elements at given sites .

12. Brainerd-Robinson ratios, showing similarity in single types at sites of different horizons . . . . . . . . . . 114

13. Brainerd-Robinson ratios, showing similarity in pottery types at single sites . 114

14. Site locations, pre-pottery, Group I . . . . . . . . . . . . . . . . 153

15. Site locations, Plain Ware, Group II . . . . . . . . . . . . . . . . . 153

16. Site locations, early black-on-whites, Group III . . . . . . . . . . . 153

17. Site locations, Reserve-Snowflake Black-on-White, Group IV . . . . . . 153

18. Site locations, Tularosa Black-on-White, Group V . . . . . . . . . . . 154

19. Site locations, Zuni Glazes, Group VI . . . . . . . . . . . . . . . . 154

20. Summary of locational preferences for entire surveyed area . . . . . . 154

21. Comparison of pollen chronologies from southern Arizona and from eastern Arizona and western New Mexico . . . . . . . . . . . . 199 


\title{
I. Architectural Details
}

\author{
By Jolin B. Rinaldo \\ Associate Curator, Department of Anthropology \\ Chicago Natural History. Museum

\section{TUMBLEWEED CANYON SITE}

The Tumbleweed Canyon site is located in east-central Arizona ten or eleven miles southeast of St. Johns and sixteen miles northwest of Springerville (Sec. 17, Twp. 11 N., R. 28 E., G. and S.R.M.). It is situated on a small mesa just below the west rim of the Little Colorado River valley (figs. 1, 2) and overlooks Lyman Reservoir. The sides of the mesa are precipitous cliffs and the entire mass of rock which forms the tableland appears to have split off from the higher rim rock to the west. This geological fault forms a small canyon about 90 feet deep and 150 feet wide. The canyon received its name from the masses of tumbleweed that drift up the slope of the west wall.

On top of the mesa were several depressions, some, in a centrally located group, outlined with basalt boulders. At either end of the ovalshaped mesa double lines of basalt boulders are piled up in what were probably walls, and other wall-like structures appear along the edge of the mesa wherever the rock talus affords a possible means of access. Numerous stone tools were found on the surface but not a single potsherd.

\section{House A}

(Figures 3 and 4)

Shape.-Roughly circular; greatest diameter, 5.4 meters.

It'alls.-Basalt boulders and gravelly light gray earth.

Floor.-Gravelly light gray earth; uneven, with rocks protruding through the surface; depth below present ground level, 20 to $45 \mathrm{~cm}$.

Firepit.-Roughly circular; sides and floor were of gravelly earth: diameter, $30 \mathrm{~cm}$. : depth, $10 \mathrm{~cm}$.

Pit.-Shallow, oval; sides and floor were of light-colored clay; contents, rocks; length, $42 \mathrm{~cm}$; width, $27 \mathrm{~cm}$. 


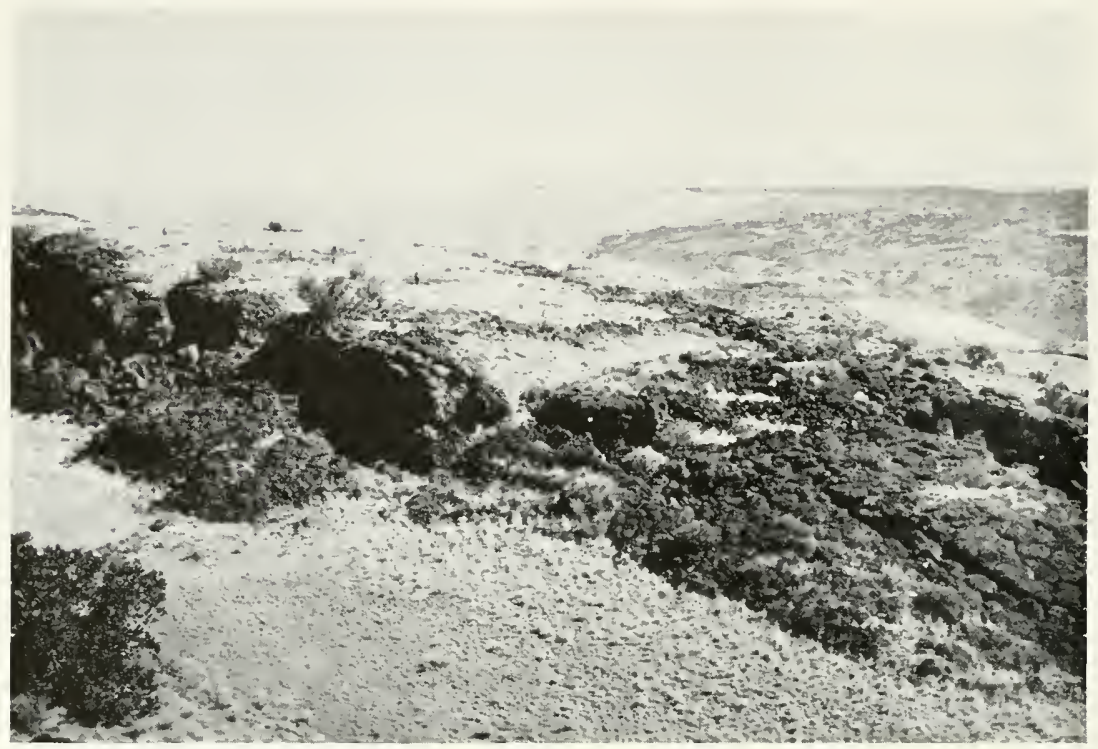

FIG. 1. Panoramic view of Tumbleweed Canyon Site and mesa from across the canyon; looking north.

Postholes.--Were spaced at irregular intervals around the edge of the house; 18 in number; average diameter, $20 \mathrm{~cm}$.; average depth, $17 \mathrm{~cm}$.

Roof.- Heavy roof timbers radiated from a point near the center of the house toward the edge of the house and toward the burned butts of posts; a layer of brush was built up on top of the rafters (thick charcoal layer), then a layer of clay.

Milling Area (fig. 5).-A cluster of metate fragments and manos was found near the firepit.

General Comments.--This house burned. An area on the south side between some of the wall rocks appears to have been an entrance.

\section{House B}

(Figures 4 and 6)

Shape.-Roughly " $D$ " shape with flat side of "D" on south; greatest inside diameter, 2.1 meters.

Walls.-Light gray gravelly earth for three walls; basalt boulders were piled up on the north side; the earthen walls sloped steeply, the north wall was closer to vertical; a lip or curb was situated at the top of the wall, 25 to $30 \mathrm{~cm}$. wide, $10 \mathrm{~cm}$. high. 


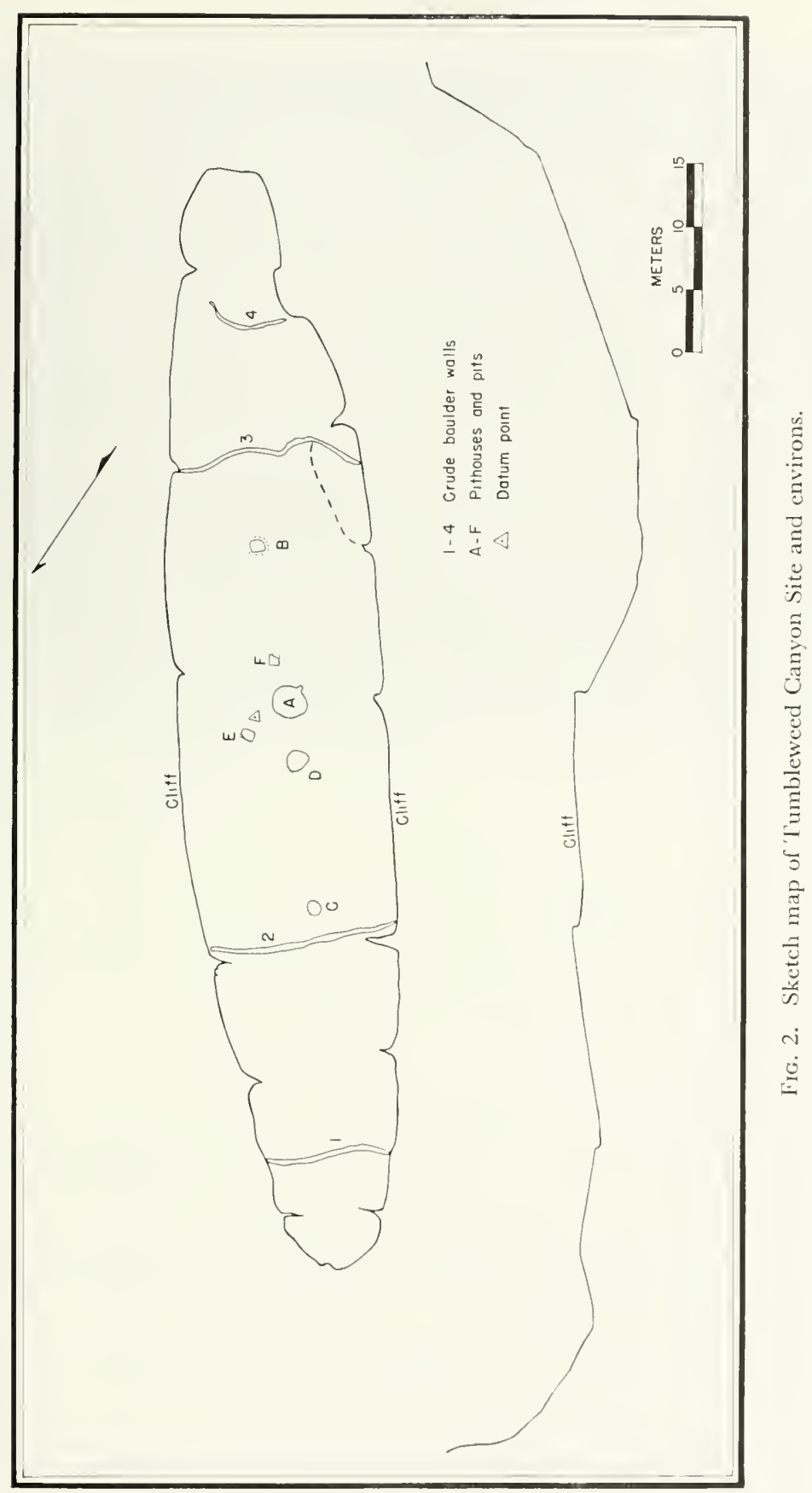

21 


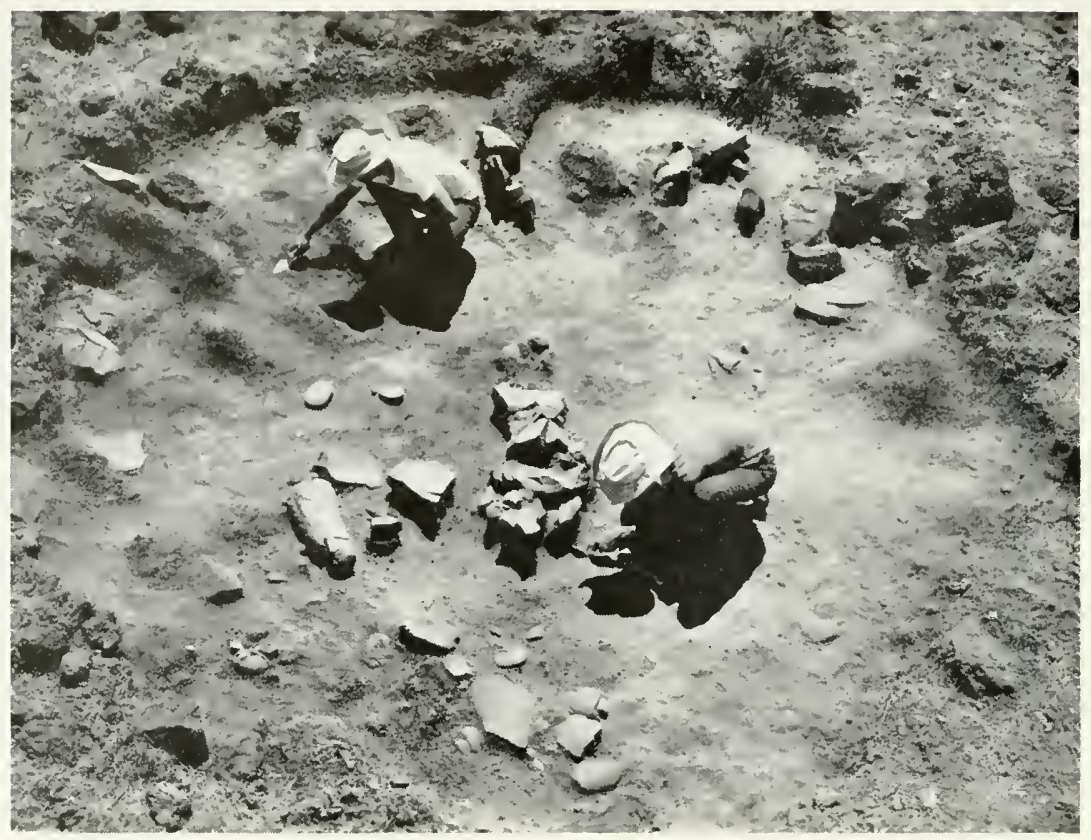

FIG. 3. House A, Tumbleweed Canyon Site, showing manos and broken metates in situ and roof beam fragments radiating toward the center of the house from the edge.

Floor.-Of gravelly earth like walls but brown in color; the surface was uneven; depth below present ground level, 32 to $65 \mathrm{~cm}$.

Firepit.-Quadrant-shaped; lined with rocks and with a rim consisting of a row of rocks; diameter, $80 \mathrm{~cm}$; depth, $8 \mathrm{~cm}$.

Pits.-None found.

Postholes.-None found.

Roof.- Height and exact character unknown; charcoal was in the fill and some burned mud but there were no impressions of roofing on the lumps of mud.

General Comments.-House B was partly burned. Malpais rocks piled up on the north side may indicate that this house was partly excavated into the bed-rock of the mesa.

\section{House $D^{1}$}

(Figures 4 and 7 )

Shape---Roughly circular; greatest diameter, 3.6 meters.

${ }^{1}$ Symbol "C" used for storage pit (fig. 2). 


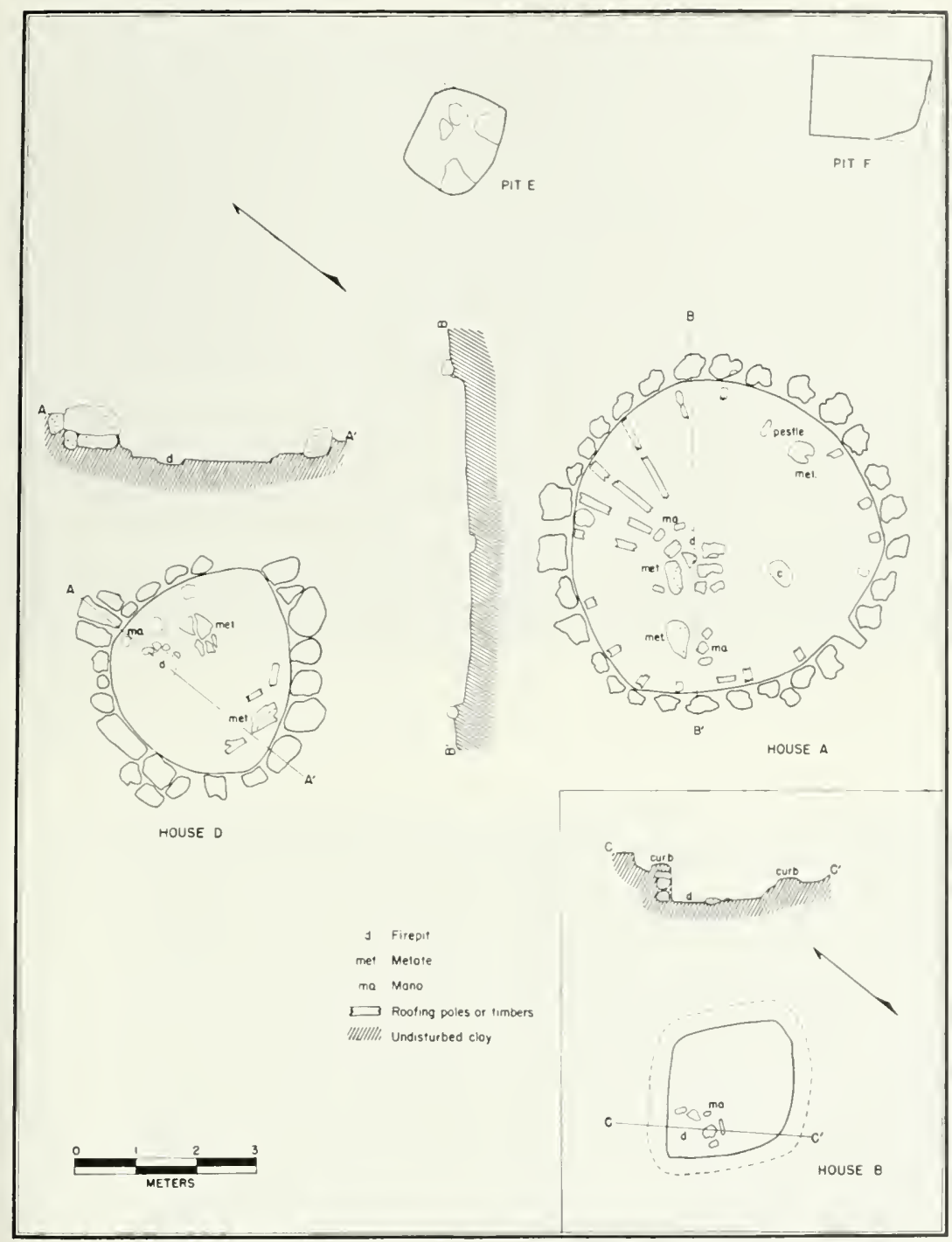

Fig. 4. Plans and sections of houses and pits, Tumbleweed Canyon Site.

IFalls. - Large and small basalt cobbles and boulders were piled up around the edge of the floor; a narrow shelf of light-colored gravelly earth was located on the south side of the house. Height of rock wall, $67 \mathrm{~cm}$.

Floor-Light gray gravelly earth and rocks; surface very uneven; depth below present ground level, $40 \mathrm{~cm}$. 


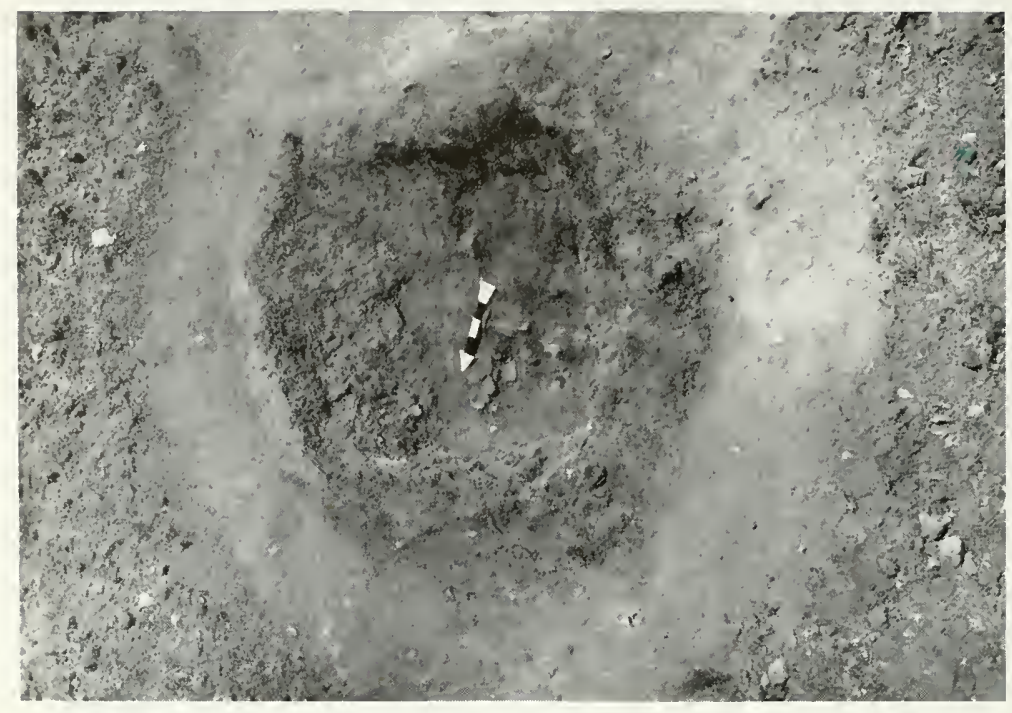

苛气

ज है

른

ป ए

च

3

坣壱

छ 00

की

可苛

气气

을

$0 \stackrel{3}{3}$

०

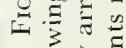

응

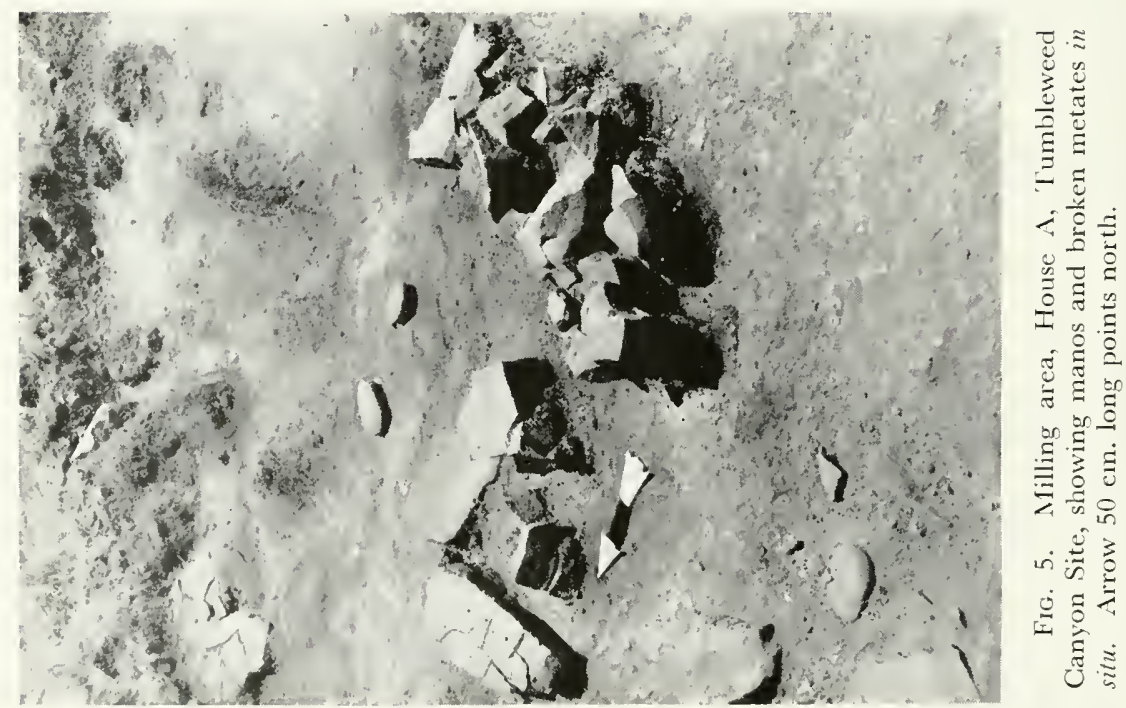




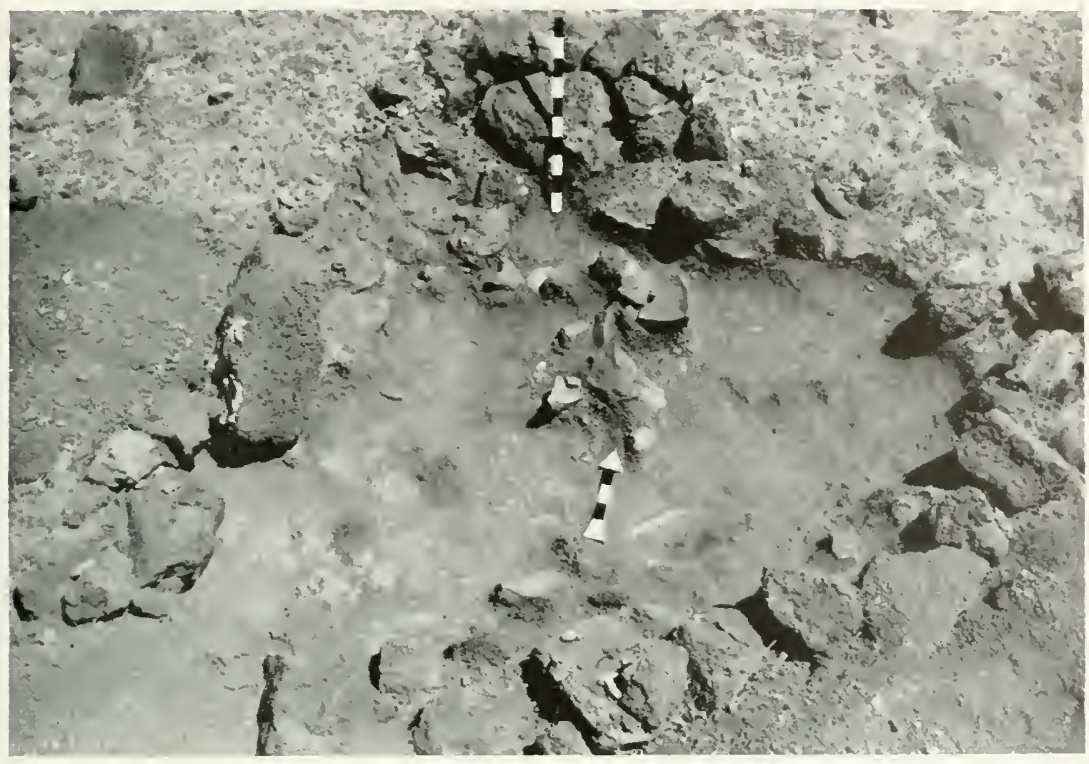

Fig. 7. House D, Tumbleweed Canyon Site, showing broken metate fragments near center of house and rocks piled up as walls around edge of house. Arrow $50 \mathrm{~cm}$. long points north; meter stick in background.

Firepit.-Roughly oval; the floor was of burned gravelly earth; rocks on the north and east sides formed a rim: length. $44 \mathrm{~cm}$.: width, $33 \mathrm{~cm}$.; depth, $7 \mathrm{~cm}$.

Postholes.-None found.

Roof.-Poles and branches crossed the angles between the larger rocks of the wall. The roof framework was covered with a layer of brush.

Milling Area.-Fragments of a metate and one mano were found by the firepit.

General Comments.-This house burned. The entrance may be indicated in the southeast quadrant by a gradual upward slope of the floor and a lower wall in this area.

\section{Storage Pits(?)}

Three roughly circular pits with vertical walls and basin-shaped bottoms were trenched, but we did not discover any definite indication as to how they were used. Two were partly excavated and one was completely excavated. All contained flint chippings, flint artifacts, and a small amount of charcoal. They were more than $190 \mathrm{~cm}$. in greatest 
diameter and from $47 \mathrm{~cm}$. to $70 \mathrm{~cm}$. in depth. Two were with the central group of houses and within 5 meters of Pithouse $\mathrm{A}$, but the third was

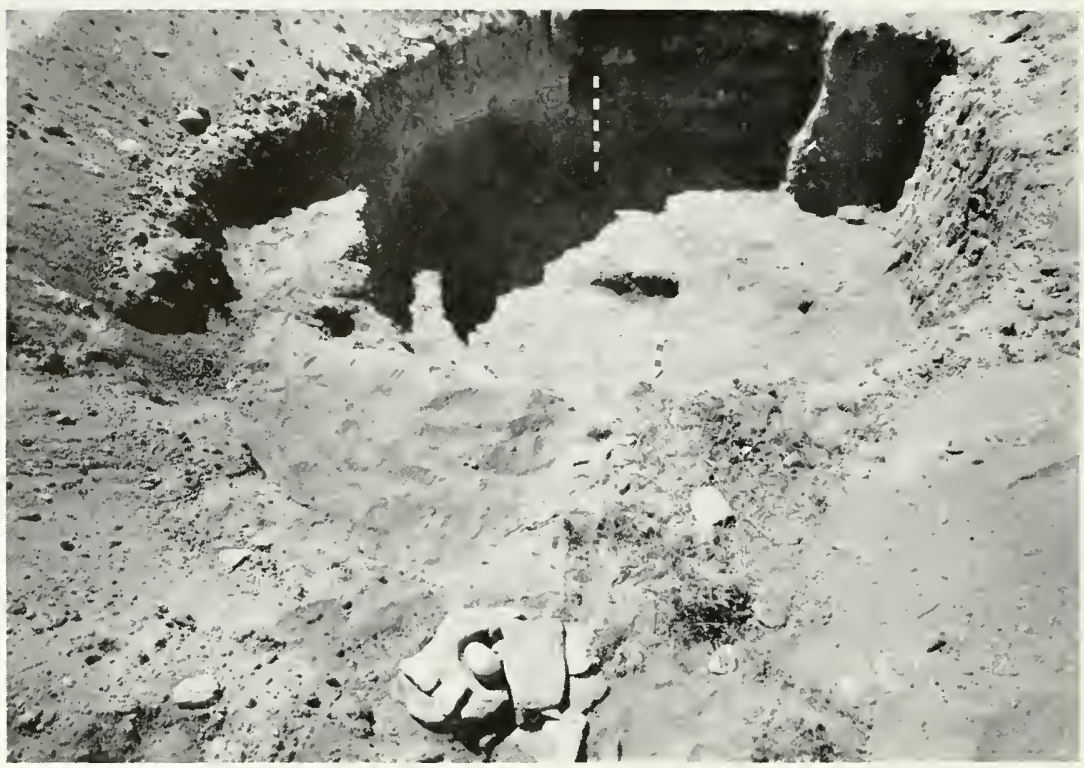

Fig. 8. Pithouse A, Goesling Site, showing postholes, firepit in center and southern recess in background. Arrow $50 \mathrm{~cm}$. long points north; meter stick in background.

more than 20 meters to the north. We have called them storage pits but this designation is simply a guess.

\section{GOESLING SITE}

(Figures 8-11)

The Goesling Site is situated on a bluff above the valley of the Little Colorado River about one mile east of the river and south of the St. JohnsSalt Lake Highway.

Shape.-The pithouses were roughly "D"-shaped, with the flat side to the south. Greatest inside diameter: Pithouse A, 5.2 meters; Pithouse B. 3.2 meters.

I'alls.-Excavated into gravelly earth. A veneer of slabs laid in abundant mortar reinforced the north and east walls of Pithouse A (fig. 10) and pebble veneer may have strengthened the north wall of Pithouse B. A single coat of plaster $0.5 \mathrm{~cm}$. thick was laid on the north wall of Pithouse $\mathrm{A}$. 
Floors.-A very thin coating of grayish adobe was spread over the compacted fill in Pithouse A. It was smooth but sloping. No floor coating was found in Pithouse B, where the floor was gravelly earth.

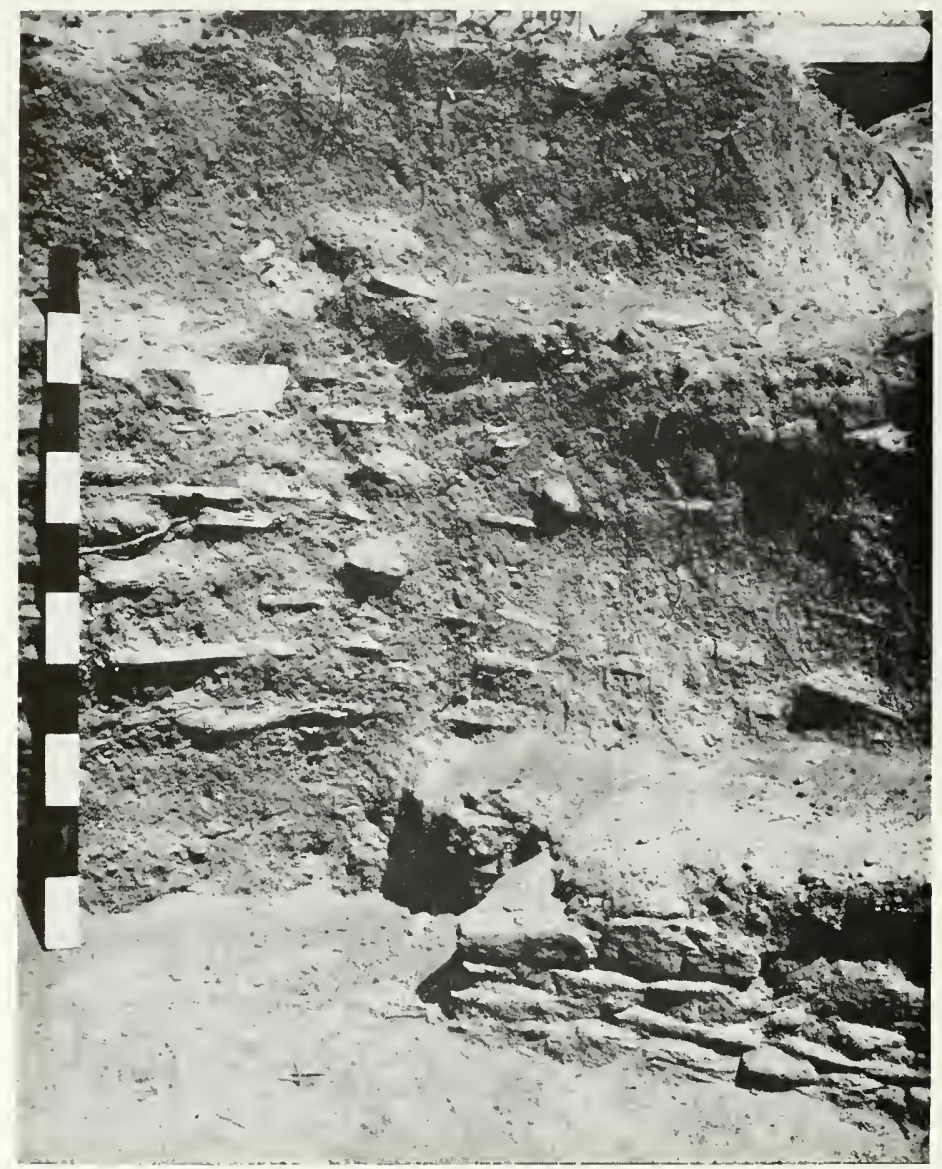

FIG. 10. Vencer masonry which reinforced north wall of Pithouse A, Goesling Site. Meter stick for scale at left.

Firepits.-One was excavated in Pithouse A. It is rectangular in shape. Length, $50 \mathrm{~cm}$; width, $40 \mathrm{~cm}$. The sides were compacted gravel mixed with a little clay. It had no definite bottom. The firepit in Pithouse B, located in the northwest corner, was a small, shallow depression which contained ashes.

Entrance--None was located. Entrance was possibly through a hatchway in the roof of Pithouse A. No entrance was found for Pithouse B. 


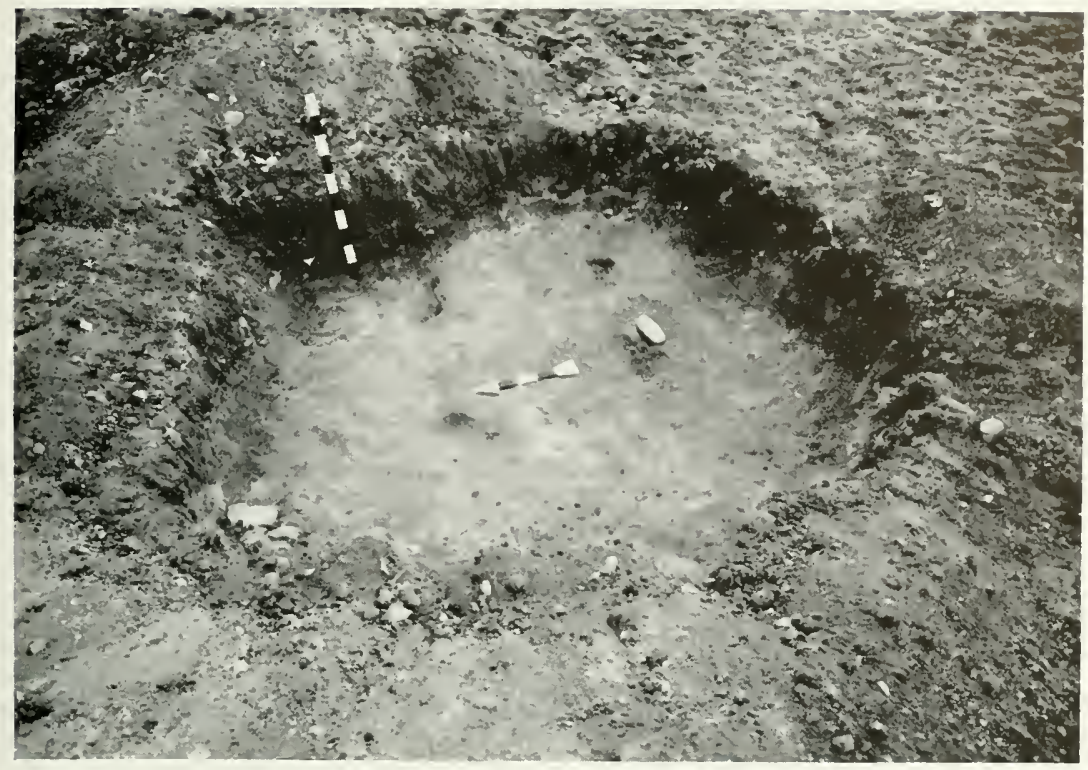

Fig. 11. Pithouse B, Goesling Site, showing shallow pit in northeast corner and quadrangular arrangement of postholes. Arrow $50 \mathrm{~cm}$. long points magnetic north; meter stick in background.

Pit.-A shallow rectangular pit was located in the northeast corner of Pithouse B. Length, $80 \mathrm{~cm}$; width, $60 \mathrm{~cm}$; depth, $10 \mathrm{~cm}$. The walls and floor were of gravelly earth. The walls were vertical.

Postholes.-Four shallow postholes were found in Pithouse B. Diameters, 20-25 cm.; depths, 8-15 cm. Four postholes were found in Pithouse A. Diameters, 20-30 cm.

Roof.-Exact character unknown. Made of heams and branches covered with adobe; evidence obtained from charcoal and burned clay.

Pottery and Artifacts. - See Chapters III and V'I.

General Comments.-These houses burned and Pithouse A was used for a pottery dump after it had been abandoned.

\section{CHILCOTT SITES}

(Figures 12-19)

The three Chilcott sites were located on the slopes of broad junipercovered hills overlooking a valley to the north. They are situated about a mile north of the highway between Concho and Show Low, Arizona (Sec. 5, Twp. 11 N., R. 25 E., G. and S.R.M.). 


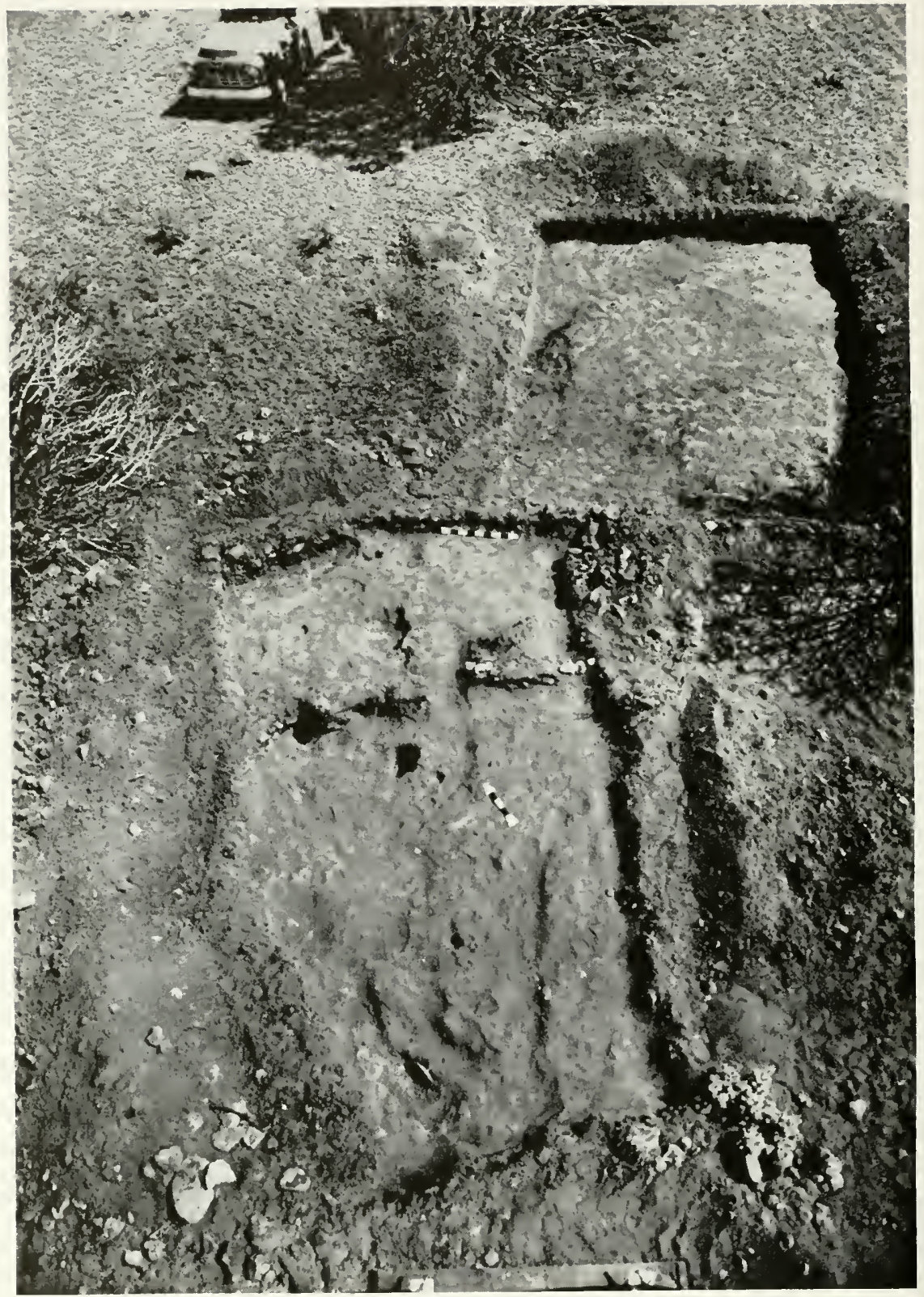

FIG. 12. Rooms 1 and 5 in foreground and Structure 2 in background, Chilcott Site 1 , showing alignment of postholes in rooms and relationship of rooms with masonry walls to larger structure. 


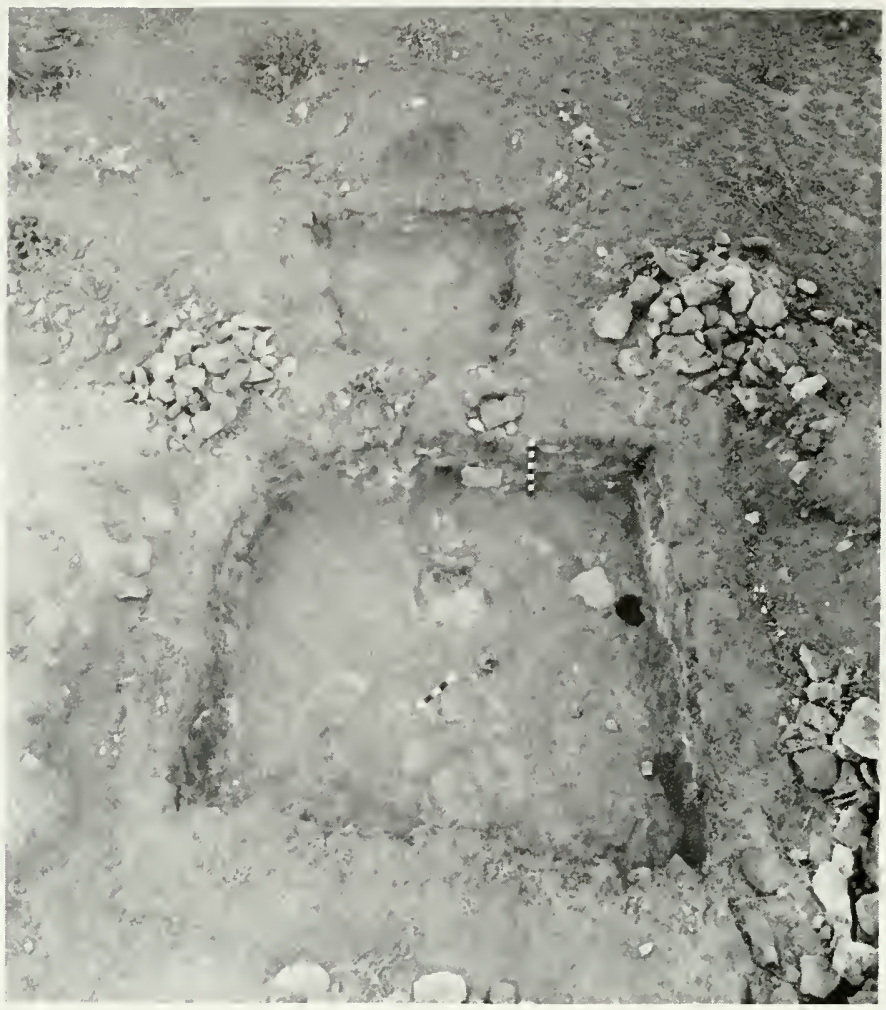

Fig. 14. Chilcott Site 1, Rooms 4 and 3, showing reduction in entryway. Arrow $50 \mathrm{~cm}$. long points magnetic north; meter stick stands against south wall of Room 4 .

\section{Chilcott Site 1}

(Figures 12-15)

Number of Rooms.-Five rooms and one structure of unknown function were excavated at Site 1. Some of these were adjacent but only two were contiguous. Most of the rooms were approximately rectangular in shape.

Dimensions of Rooms. - Rooms range in length from 2.6 to 4.4 meters and in width from 1.8 to 4.1 meters. The portions of the walls that remain standing are 35 to $100 \mathrm{~cm}$. high (including excavation into native soil).

11 alls.-Floors were excavated into white soil or red clay $5 \mathrm{~cm}$. to $170 \mathrm{~cm}$. below the surface. Masonry was based on the old ground surface above the floor; there were no prepared foundations. The upper walls were constructed of crude random rubble type masonry made of assorted unshaped basalt boulchers and smaller stones. Some through 


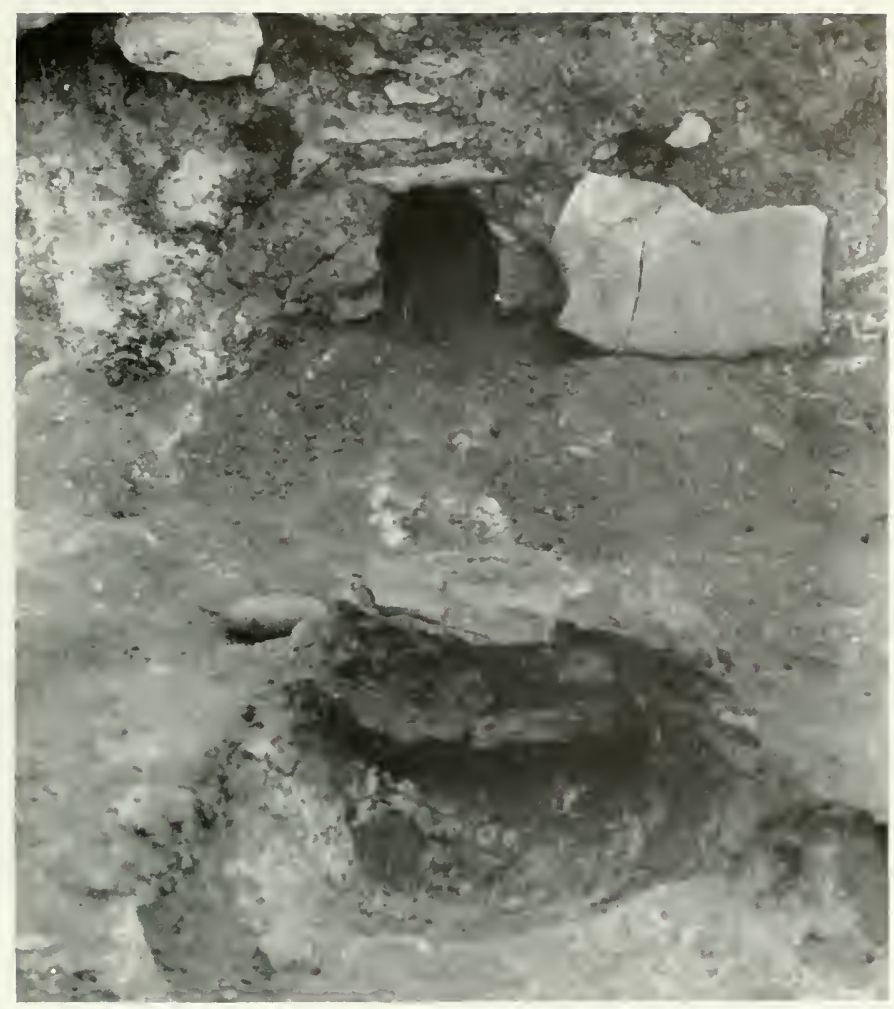

FIg. 15. Detail of firepit, deflector, ventilator opening and damper slab, Room 4, Chilcott Site 1.

stones appeared in the thinner partition wall between Rooms 1 and 5: also, short lengths of wall were of composite construction, with two facings. These portions were built with two rows of stones and were $30 \mathrm{~cm}$. thick; others had only a single row of stones and were $20 \mathrm{~cm}$. thick. The large number of fallen wall stones indicates that the walls extended a few feet above their present height.

Mortar: A brown adobe or mud mixture which had dried mediumhard.

Plaster: A single coat of undecorated mud plaster up to $2.0 \mathrm{~cm}$. thick had been applied to the walls.

Floors.-Tan native soil or white rock was covered with a layer of gray to brown adobe clay: the surface is generally uneven, some sections are smooth. The earlier floor in Room 4 had been partly removed and the postholes in it covered by a later floor. 
Firepits.-Three were found, in Rooms 1, 4, and 5. The one in Room 1 was round, was lined with adobe and was $60 \mathrm{~cm}$. in diameter and $20 \mathrm{~cm}$.

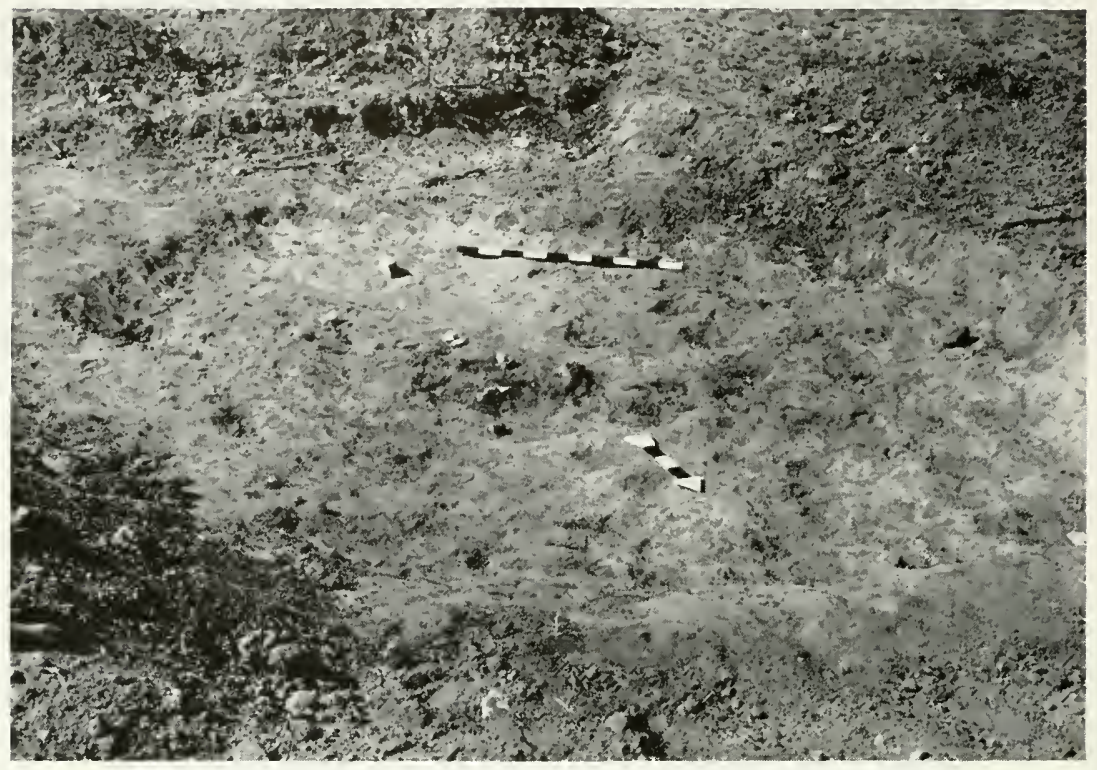

FIG. 16. View of Room 1, Chilcott Site 2, showing uneven floor and area of burned post and rocks near center of structure. Arrow $50 \mathrm{~cm}$. long points magnetic north; meter stick in background near test trench.

deep. The one in Room 4 was rectangular, with floor and walls of white rock except on the south side, where the wall was lined with adobe clay. A large stone formed the rim on the north, a masonry deflector on the south (fig. 15); this pit was $65 \mathrm{~cm}$. long, $60 \mathrm{~cm}$. wide, and $22 \mathrm{~cm}$. deep. The one in Room 5 was rectangular and was lined with adobe plaster; it was $60 \mathrm{~cm}$. long, $50 \mathrm{~cm}$. wide, and $12 \mathrm{~cm}$. deep. All these firepits contained white ash. Those in Rooms 1 and 4 were near the center of the room; that in Room 5 was in an angle of the north wall.

Pit.-One in the east end of Room 5 was roughly bell-shaped, and the bottom was lined with stone slabs. Diameter at mouth, $35 \mathrm{~cm}$., at floor level, $45 \mathrm{~cm}$; depth, $35 \mathrm{~cm}$.

Postholes. - Two postholes were aligned down the long axis of Room 5; another in Room 1 may possibly be an extension of this row. Diameters, $10,11,13 \mathrm{~cm}$; depths, 10,13,15 cm. Four postholes in the north half of Room 3 near the walls were $18-30 \mathrm{~cm}$. in diameter and $10 \mathrm{~cm}$. deep. Four postholes were also found in Room 4; the two later ones were 15 and 


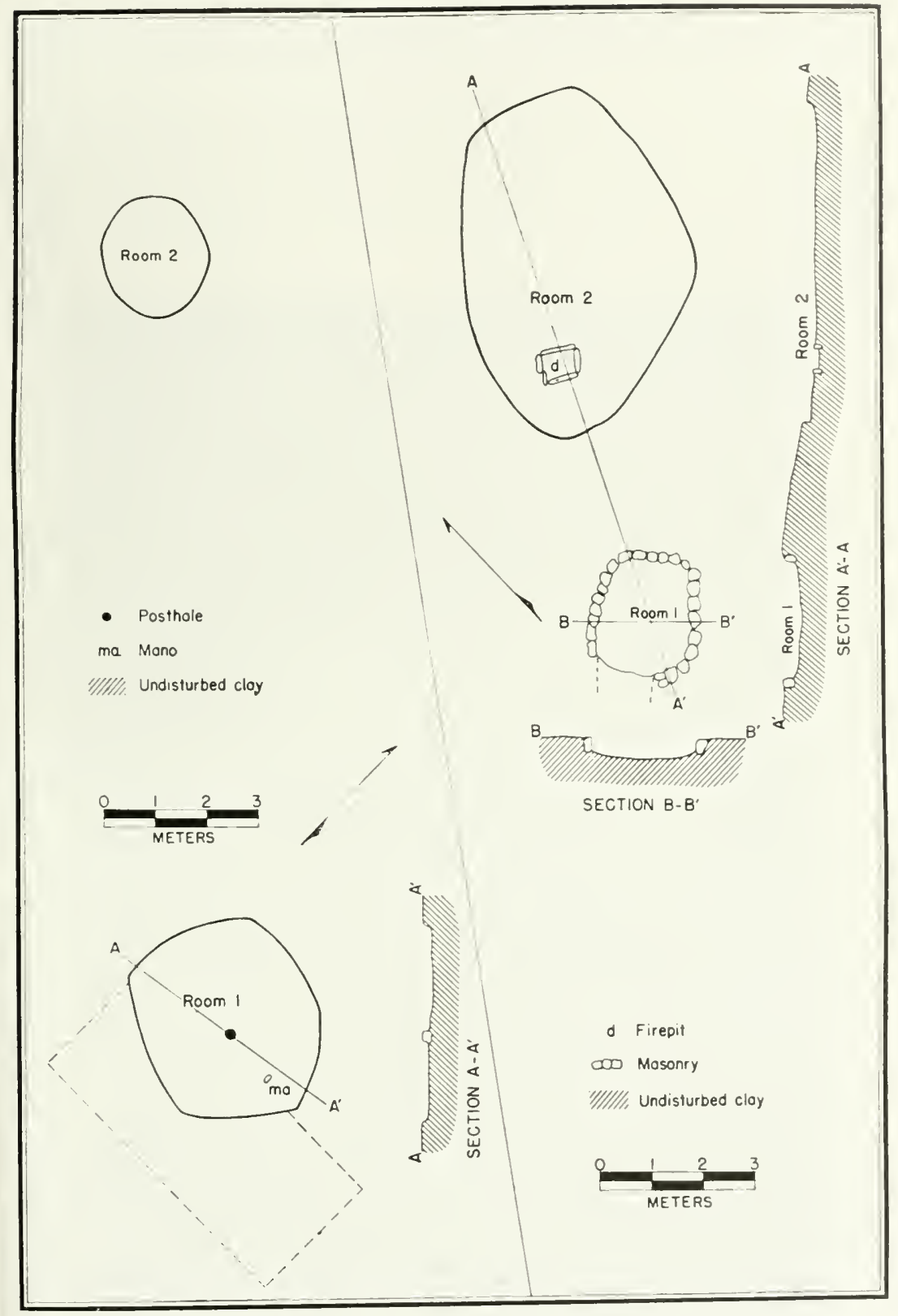

Fig. 17. Plans and sections of Chilcott Site 2 (left) and Site 3 (right). 
$31 \mathrm{~cm}$. in diameter, 25 and $33 \mathrm{~cm}$. deep; one was recessed in the east wall, the other was next to the west wall. The two earlier ones were 28 and $35 \mathrm{~cm}$. in diameter, 48 and $50 \mathrm{~cm}$. deep.

Ventilator (fig. 15). - In the center of the southeast wall; crude rubble masonry based at floor level reduced the width of a former passage to an "antechamber" (Room 3) from $55 \mathrm{~cm}$. down to $20 \mathrm{~cm}$., and in height down to $30 \mathrm{~cm}$. It had a stone slab lintel and masonry sides. It was closed with a stone slab "damper."

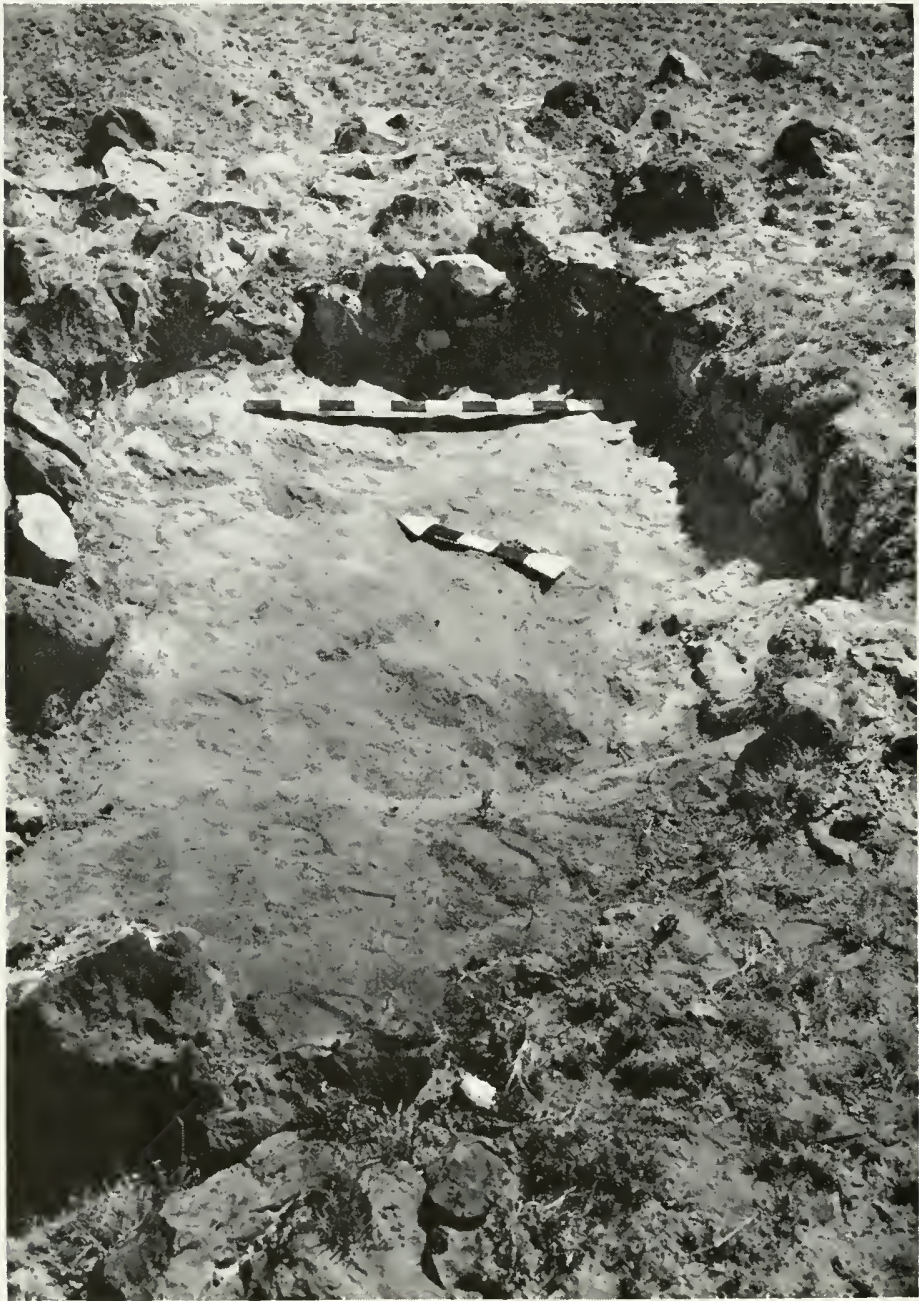

FIG. 18. Room 1, Chilcott Site 3, showing basalt boulder walls and general shape of structure. Arrow $50 \mathrm{~cm}$. long points magnetic north; meter stick in background. 
Roof.-Evidence from burned clay impressions and charred fragments. Made of beams and split poles or splints covered with clay. The main beams or girders were supported by posts.

General Comment.-Rooms 3 and 4 appear to have been the antechamber and main room of a single pithouse (fig. 14) which had been remodeled into two rooms. A new floor had been added in both sections covering the postholes in Room 4. and the passageway between the two rooms had been converted into a ventilator. The butts of wood posts in these rooms were charred. Apparently these posts were obtained from trees that were felled by burning them at the base.

\section{Chilcott Sites 2 a.id 3}

(Figures 16-19)

Number of Structures. - Two structures were excavated at each of these sites. Trenching and testing in the general sherd area failed to reveal additional structures.

IValls.-Only one structure (Room 1, Site 3) had masonry walls. These were of random rubble of boulders laid with the flat side to the interior of the room. Dimensions of stones: length. 22-30 cm., average, $26 \mathrm{~cm}$.; thickness. 15-25 cm., average. $19 \mathrm{~cm}$.: width. 3-4 cm. less than length. They were laid in abundant mud mortar. No plaster was found. Dimensions of walls: present height (including earth base), $45-50 \mathrm{~cm}$.; width, $27-30 \mathrm{~cm}$.

Floors.-Excavated below the old ground level. This soil was compacted and formed the floors which sloped toward the center of the room and were uneven.

Firepit.-One firepit. located in the south end of Room 2, Site 3, was rectangular in shape and lined with rough stone slabs. Length, $63 \mathrm{~cm}$; width, $54 \mathrm{~cm}$; depth, $10 \mathrm{~cm}$.

Postholes.-One posthole, at the north end of Room 2. Site 3, was $15 \mathrm{~cm}$. in diameter; it contained rotten wood. One near the center of Room 1. Site 2, was $30 \mathrm{~cm}$. in diameter; it contained a charred post.

Roof.-Exact character unknown. Approximate limits of rooms at Sites 2 and 3 were determined by dark soil containing minute charcoal fragments: it is assumed these dwellings were shelters roofed with brush.

Shape.-The three shelters were approximately oval in shape, 4.0 by 3.7 meters, 2.2 by 2.1 meters, 5.75 by 4.75 meters. The room with masonry walls was roughly rectangular in shape, 2 meters long by 1.75 meters wide. 


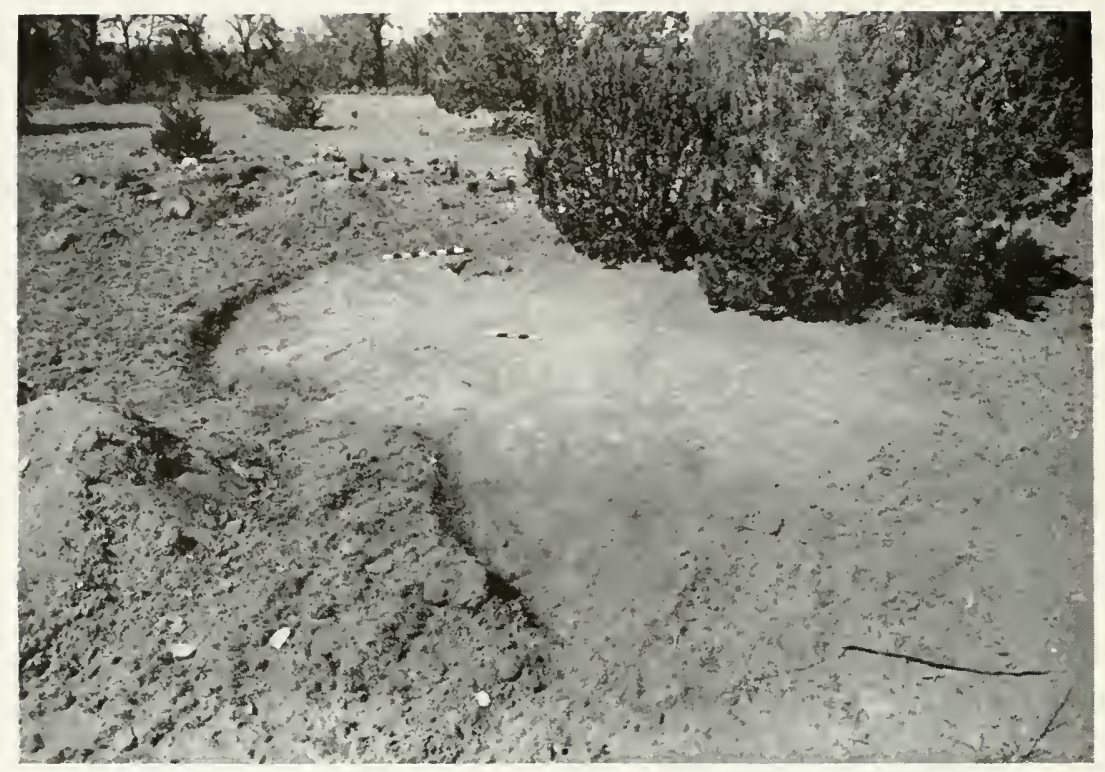

FIG. 19. Room 2, Chilcott Site 3, showing rectangular firepit in background near meter stick. Arrow $50 \mathrm{~cm}$. long points magnetic north.

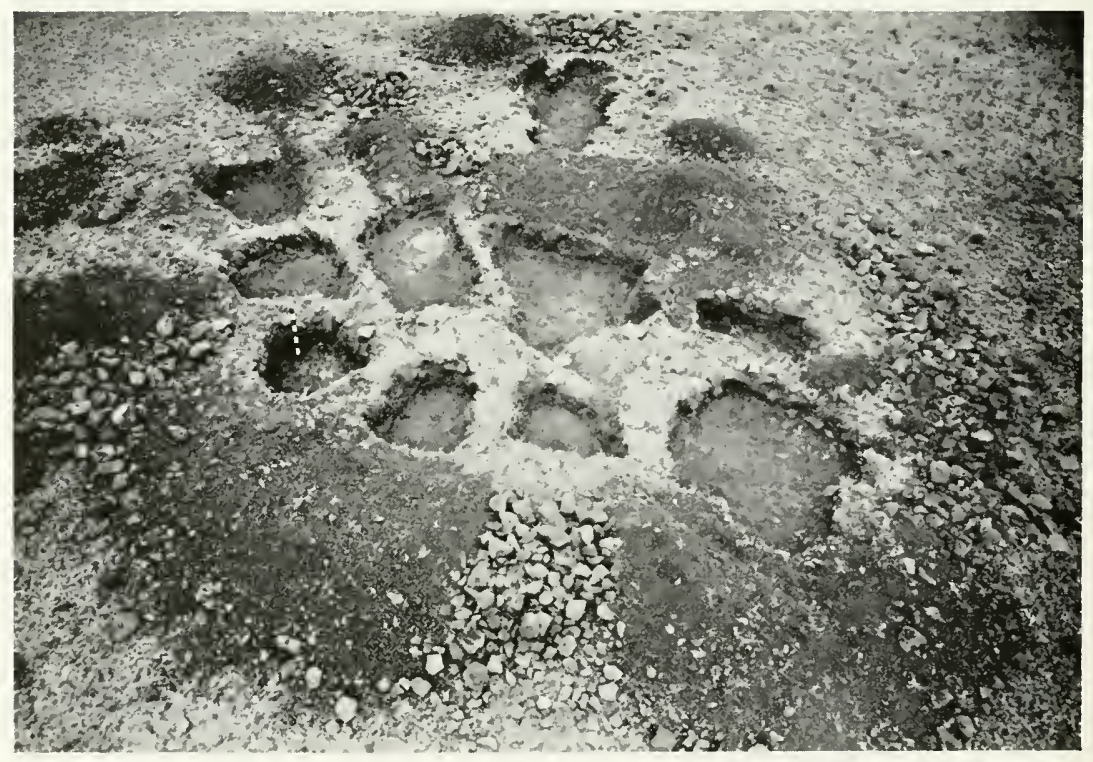

Fig. 20. Thode Site. Arrow $50 \mathrm{~cm}$. long points magnetic north and to firepit in Room E; meter stick at left. 


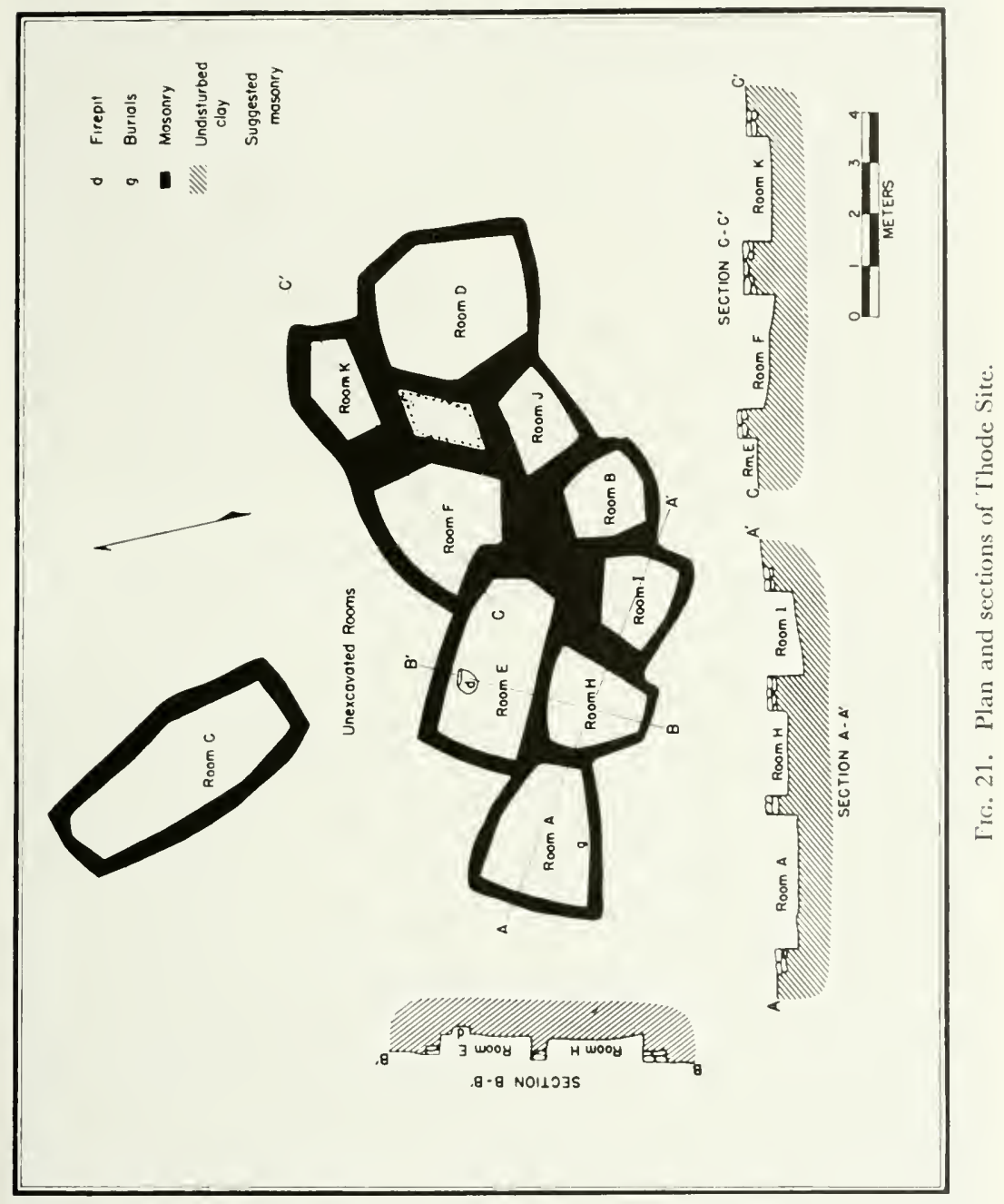




\section{THODE SITE}

(Figures 20 and 21)

This small site is situated on the west bank of the east fork of Mineral Creek about $21 / 2$ miles east of Vernon, Arizon?, on U. S. Highway 60 (Sec. 13, Twp. 10 N., R. 25 E., G. and S.R.M.). Before excavation the site appeared as a low mound of rocks about 200 yards south of the highway and west of a grove of oaks that grow in the creek bottom.

Number of Rooms. - Eleven rooms were exeavated. Two or three more may be present in the north end of the ruin. Several of the rooms are adjacent but not contiguous (see fig. 21).

II'alls. - Crude masonry composed of assorted sizes of unshaped cobbles and boulders all of igneous rock and ranging in length from $8 \mathrm{~cm}$. to $20 \mathrm{~cm}$. The thinner walls contain some through stones. The greatest height of the standing wall, including the earthen base, was $67 \mathrm{~cm}$; the base of the masonry was on the old ground surface, 25 to $55 \mathrm{~cm}$. above the floor. The mortar was mud: some stone-to-stone contact was observed in the bedding planes: no plaster was found.

Floor.-Generally dark red clay native to the locality; light-colored soil in some areas: the surface was fairly even but sloping. The floor levels were semi-subterranean.

Firepit.-One in Room E. "D"-shaped with flat (east) side made of a stone slab set on edge; length, $45 \mathrm{~cm}$.; width, $40 \mathrm{~cm}$.; depth, $35 \mathrm{~cm}$. It was lined with native clay. Small areas of burned floor and light gray ashes (not contained in pits) were found in two other rooms (Rooms C and F). These may have been hearth areas.

Entrances.-None found.

Pits.-None found.

Postholes.-None found.

Roof.-Exact character unknown. Charred pole fragments were found. The roofing poles may have been supported by the walls.

General Comments.--This site has the appearance of a series of shallow pithouses or deep sub-surface rooms clustered about a nucleus (Room E).

\section{RIM VALLEY PUEBLO}

(Figure 22)

Situated on a mesa above the west bank of the Little Colorado River about four miles north of Springerville, Arizona. Rim Valley Pueblo was built on a little flat about four-fifths of the distance from the rim-rock 


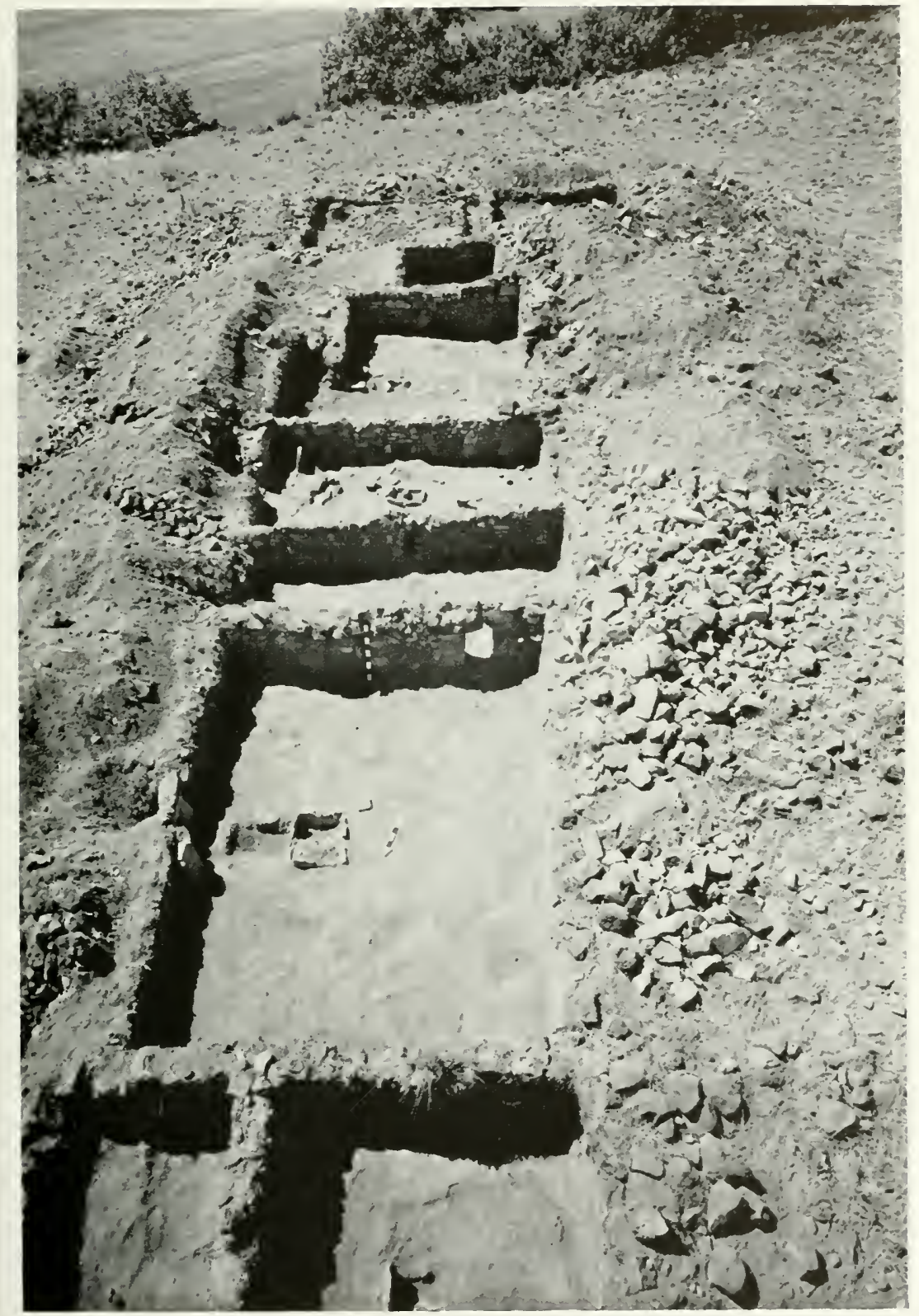

FIG. 22. Rim Valley Pueblo, looking south. Room A contains arrow $50 \mathrm{~cm}$. long pointing magnetic north and meter stick standing against the south wall. 


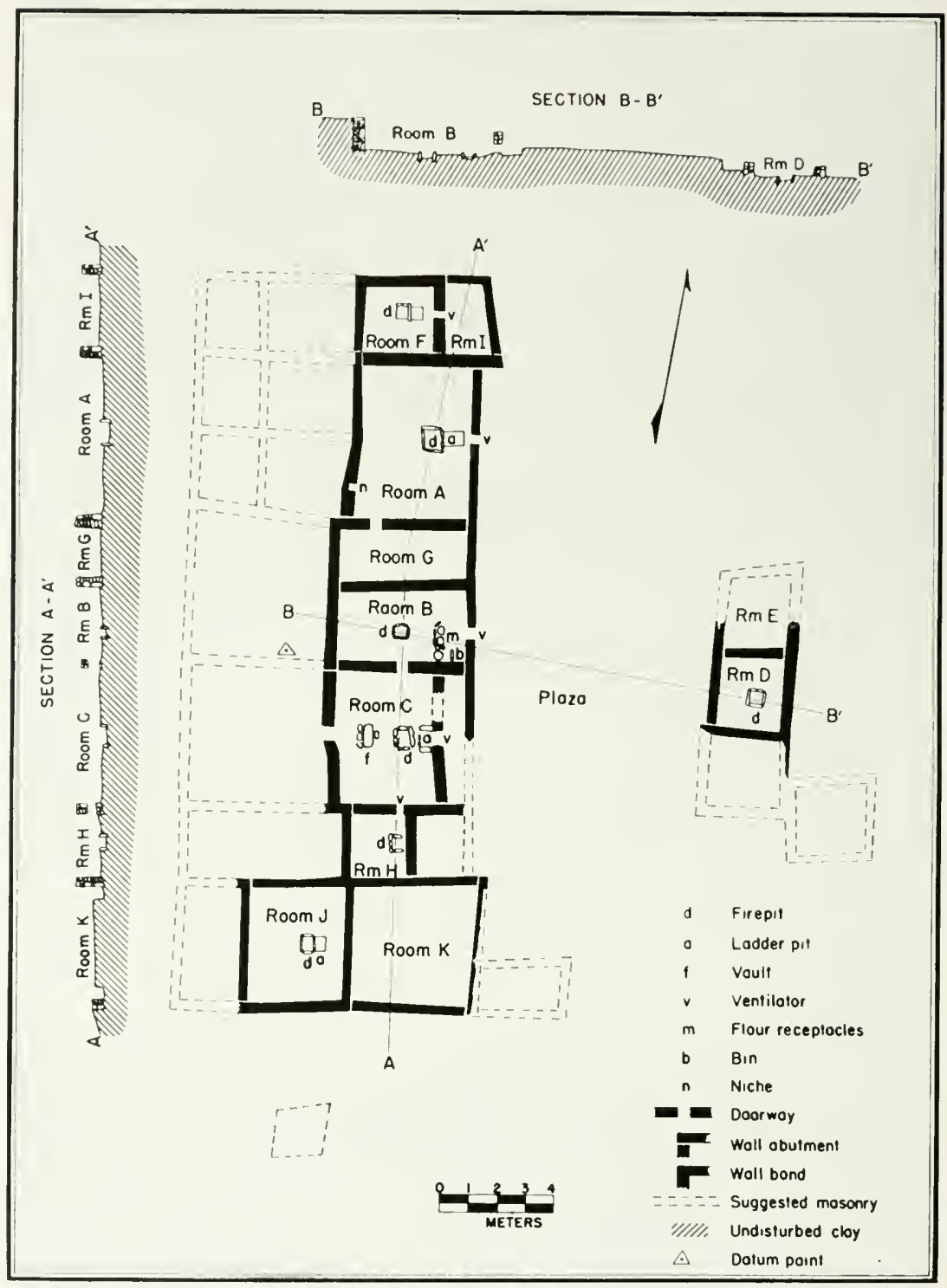

Fig. 23. Plan and sections of Rim Valley Pueblo. 


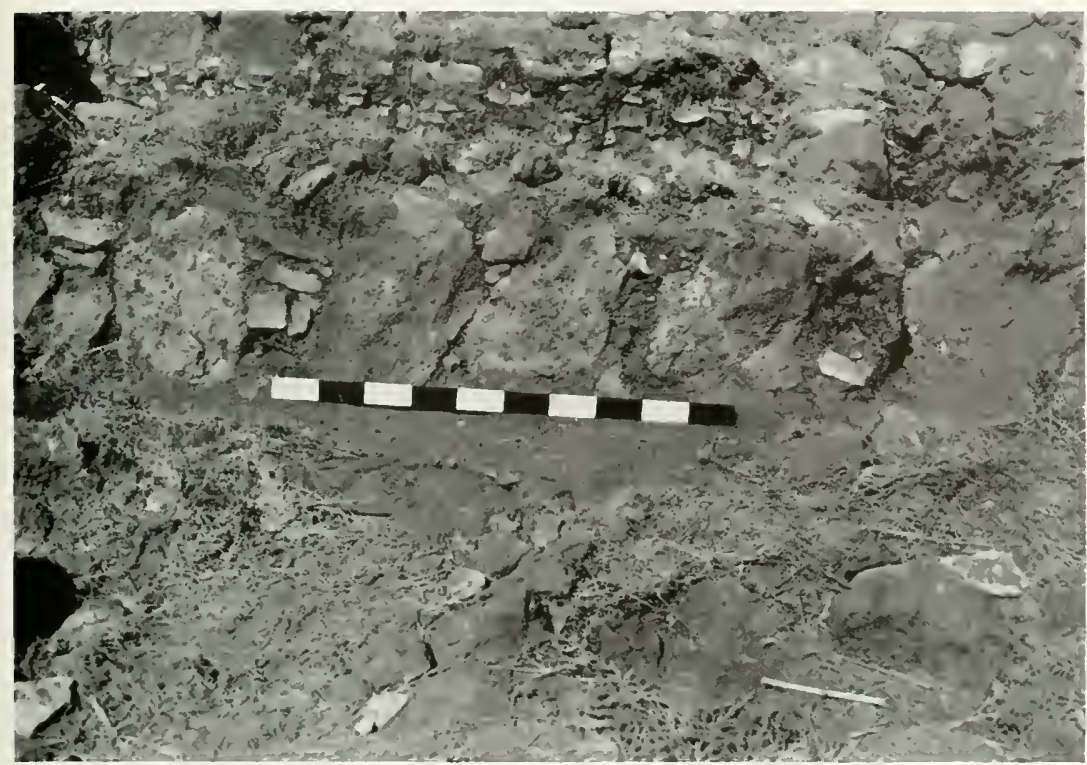

FIG. 24. Outer wall of Room I, Rim Valley Pueblo. Meter stick in foreground.

of the cliff to the top of the mesa (NE $1 / 4$, SW $1 / 4$, Sec. 8, Twp. $9 \mathrm{~N}$., R. 29 E., G. and S.R.M.). The Hooper Ranch Pueblo (see p. 53) lies a few hundred yards below to the northeast on the opposite bank of the river.

Arrangement of Pueblo Parts.-Rooms are grouped in two units of one story each, on opposite sides of a plaza (fig. 23). The unit on the east side is small, has only four rooms, is roughly L-shaped and only one room wide. A larger unit on the west side is rectangular in form and two or three rooms wide.

Number of Rooms,--Eleven rooms were excavated and fourteen more were indicated by the outlines of fallen walls; an estimated twenty-one rooms are in the larger unit.

Dimensions of Rooms.- Roughly rectangular in shape; length, 2.405.65 meters, average, 3.83 meters; width, 1.50-4.50 meters, average, 2.74 meters.

II alls.-No prepared foundations; walls were based on bedrock, on native soil a few centimeters above bedrock, or, in one instance, on trashy fill. Masonry started a few centimeters below floor level.

Types of Masonry: Some through stones were used, but most walls were a product of two facings, each of a different type of masonry built up one against the other and with some stones interlocking inside the wall. 
Type 1 was similar to vertical slal) masonry. A course of large stones (average $36 \mathrm{~cm}$. long. $24 \mathrm{~cm}$. wide, 2 or $3 \mathrm{~cm}$. less thick than wide) alter-

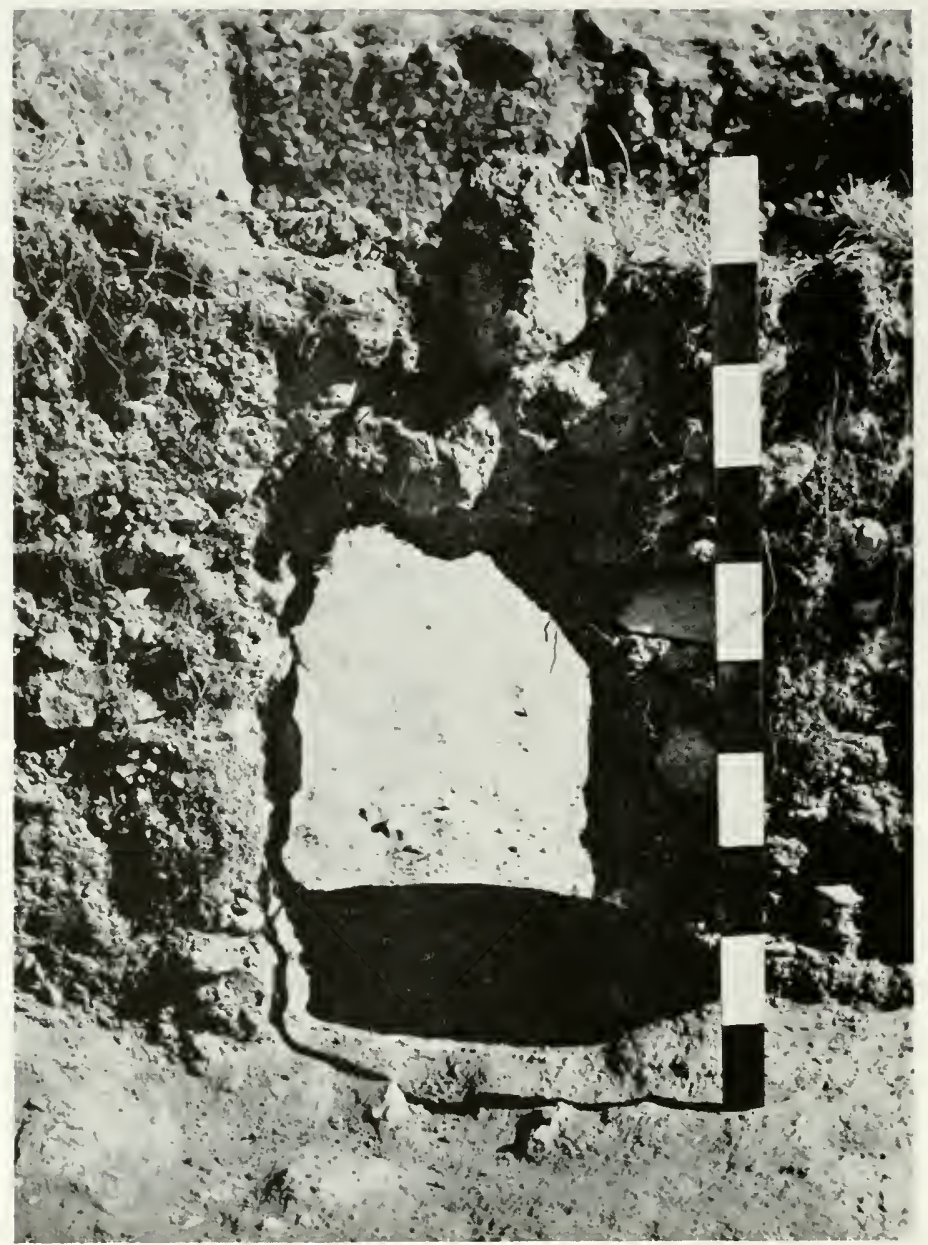

FIG. 25. Rectangular doorway in north wall of Room G, Rim Valley Pueblo. Meter stick at right.

nated with several courses of smaller stones including some small slabs (average $10 \mathrm{~cm}$. long, $8 \mathrm{~cm}$. wide, $6 \mathrm{~cm}$. thick). Small slabs and stones were used as chinking in the vertical joints between the larger stones. The outer surface of the exterior walls was generally of this type (fig. 24).

Type 2 was random rubble masonry of cobble-sized stones laid up in abundant mud mortar. The courses sloped, the stones used were only 


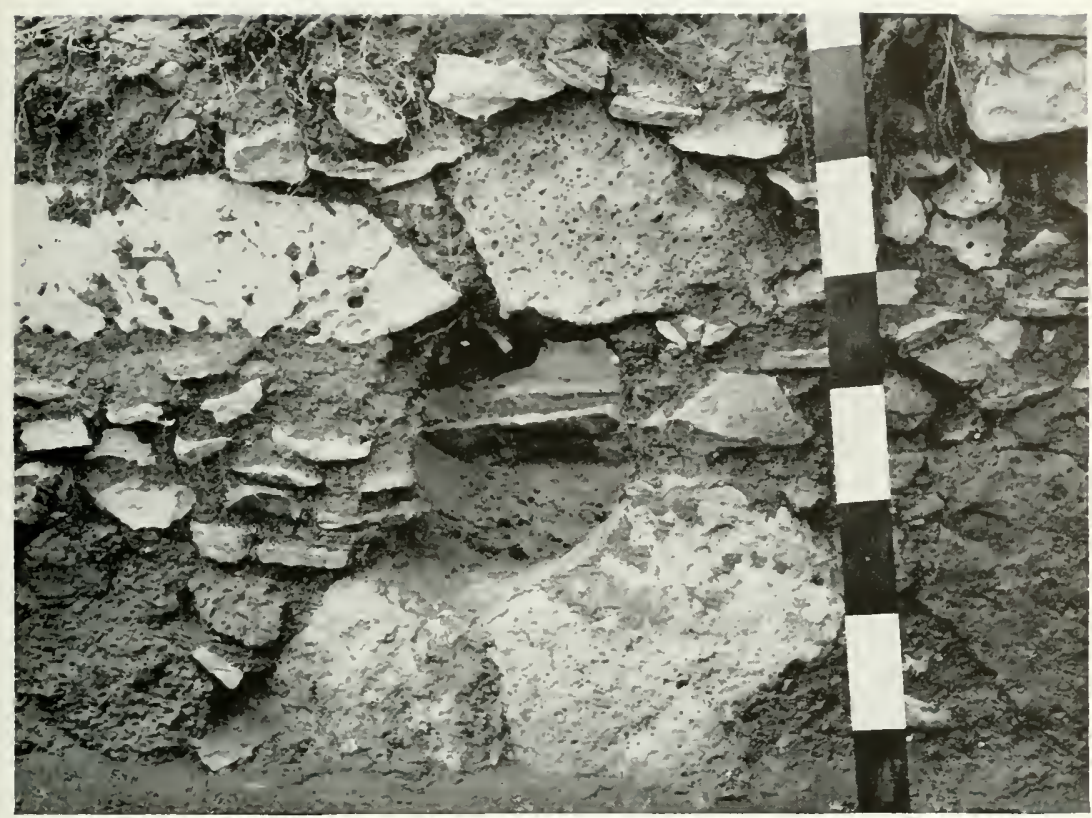

Fig. 26. Oval ventilator in south wall of Room C, Rim Valley Pueblo. Meter stick at right.

roughly matched for size, and the general product was crude in appearance. This type of masonry was generally used for the surfaces of walls visible from the interior of the room; usually they were covered by plaster. With the exception of one smaller room (Room $G$ ), each room had two walls with each type of masonry. Generally the south and west walls were of Type 1, the north and east walls of Type 2.

Dimensions: Walls ranged in thickness from 25 to $38 \mathrm{~cm}$. (average, $31 \mathrm{~cm}$.): present wall heights ranged from 30 to $110 \mathrm{~cm}$. (average, $75 \mathrm{~cm}$.).

Most often basalt boulders and cobbles from local outcroppings were used as stones in the wall. Miany were angular in shape, some rounded. Less often walls were constructed of laminated sandstone slabs and angular chunks of sandstone probably quarried from nearby cliffs.

Small flat or angular pebbles were infrequently inserted in the mud mortar of the walls to fill voids.

The mortar was soft gray mud of fine texture, occasionally containing larger particles.

The plaster was gray or brown in color. undecorated, quite thick. It was applied in a single coat and smoothed over irregularities in the surface of the masonry. 


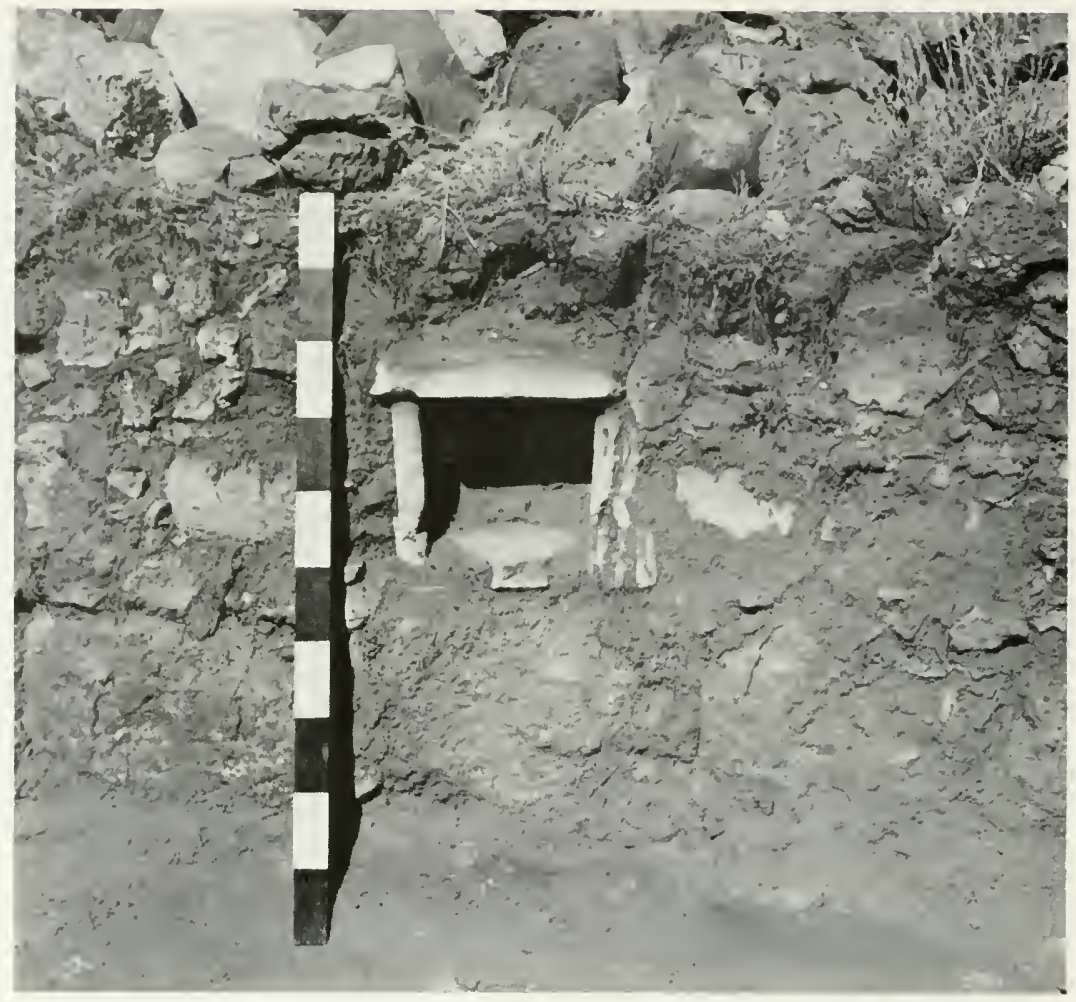

FIG. 27. Niche in north wall of Room A, Rim Valley Pueblo. Meter stick at left.

Doorways (fig. 25): Both doorways were rectangular: neither had been sealed and both were through interior (partition) walls. Width, 36 and $45 \mathrm{~cm}$.: probable height (lintel fallen in), 59 and $60 \mathrm{~cm}$; sills were adole plaster or stone slabs; the sides were masonry and the lintel (one lost) was a stone slab.

Tentilators and Jiches (figs. 26, 27).- Six ventilator openings were found, all in the centers of walls and opposite firepits. Five were rectangular in shape and with their sills near floor level and one was oval in shape and with its sill $20 \mathrm{~cm}$. above the floor. Four had lintels of stone slabs set on end. The others had sides of Type 1 masonry on one side of the wall with large boulders forming the sides of the opening. The sill of the oval opening between Rooms $\mathrm{H}$ and $\mathrm{C}$ was formed by a semicircular are carved(?) in a thick slab-like boulder (fig. 28). The sills of the rectangular openings were adobe clay or slabs covered with adobe. The openings ranged in width from 20 to $35 \mathrm{~cm}$. (average, $29 \mathrm{~cm}$.), in height from 20 to $33 \mathrm{~cm}$. (average, $29 \mathrm{~cm}$.). 


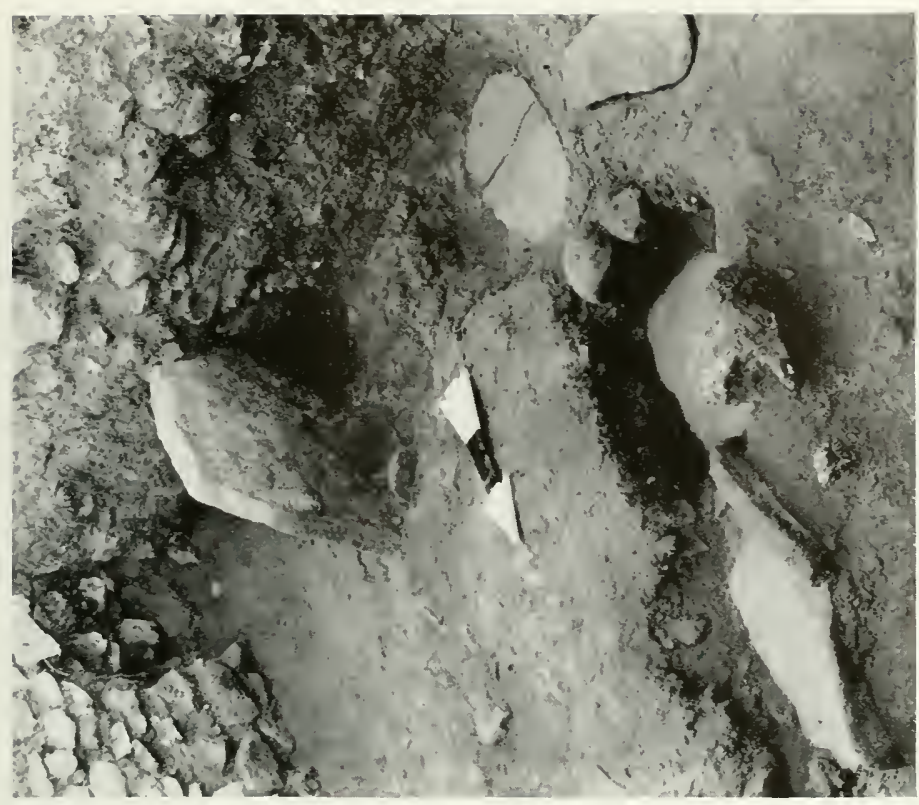

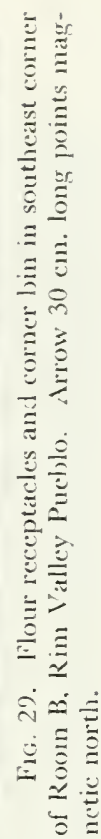

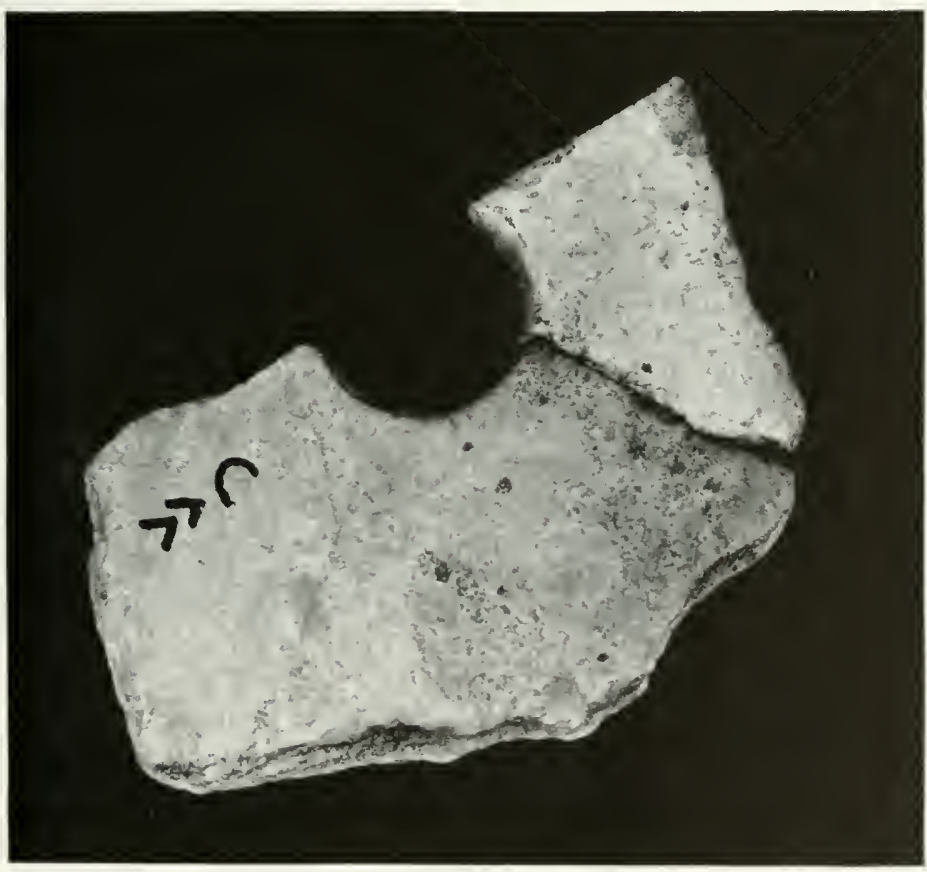

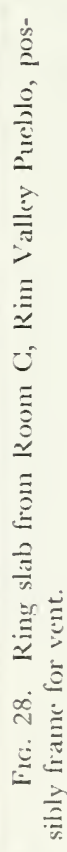


One niche was found in the west wall of Room A, $50 \mathrm{~cm}$. above the floor near the southwest corner. The heighe of the opening was $22 \mathrm{~cm}$.; the width, $24 \mathrm{~cm}$; the depth, $24 \mathrm{~cm}$. The lining was stone slabs except the back, which is adobe plaster.

Floors. Material: A layer of smooth adobe clay was laid 2.0 to $6.0 \mathrm{~cm}$. thick over gray basalt bedrock or gravelly earth. The adobe curved up to meet the wall plaster.

Alterations: The vault in Room C was corered with plaster matching the floor. Part of the floor in Room A was refinished with a thin layer of adolse.

Flour receptacles: Three flour receptacles (fig. 29) were found in the southeast quadrant of Room B near the bin. Lengths, 34, 30, $40 \mathrm{~cm}$; widths, 28, 30, $33 \mathrm{~cm}$; depths, $12,10,6 \mathrm{~cm}$. They have bottoms made of stone slabs, one of which was smooth and was used as a metate; they were separated by walls of slabs or manos set on edge or by rows of stones. Manos and hammerstones were found nearby.

Firepits (fig. 30).-Rectangular in shape, with stone-slab-lined sides and bottoms: the slabs on the sides projected above the floor level. The slab on the side toward the ventilator was slightly higher (example, Room F) than the others. The firepits have gravel or bedrock bottoms (examples, Rooms B and C). The firepit in Room C was made of rough slabs; in Room B the slabs were finished more smoothly. Firepits were generally located in line with the ventilators near the centers of the rooms. Length, 42-75 cm. (average, $49 \mathrm{~cm}$.); width, 33-62 cm. (average, $40 \mathrm{~cm}$.); depth, 20-25 cm. (average, $20 \mathrm{~cm}$.). Some firepits have notches in their sides and gaps at their corners. All the firepits contained fine white ash and a small amount of charcoal. The one in Room B held a pot rest stone standing at the center of the south side.

Ladder-pits(?).- Rectangular ladder pits(?) (fig. 30) lined with adobe plaster and in one instance rimmed with slabs adjoined the firepits in Rooms A, C, F and J. They are on the side toward the rentilator (east side). Lengths, 69, 45, 40, $45 \mathrm{~cm}$.: widths, 45, 44, 40, $35 \mathrm{~cm}$.; depths all $20 \mathrm{~cm}$.

I'ault.- A vault (fig. 31) was found near the west wall of Room C in line with the firepit, the ventilator and the door; it had been excavated into bedrock and lined with two courses of masonry on the west side, one slat) at each end, and one at the east side below floor level. Rectangular in shape. Length, $75 \mathrm{~cm}$.; width, $36.0 \mathrm{~cm}$; depth, $25 \mathrm{~cm}$. It had been covered with a later floor. It contained an awl-sharpening stone.

Bin.-A large slab set on edge projected from the south wall of Room B. making a bin (fig. 29) with three sides in the southeast corner. 


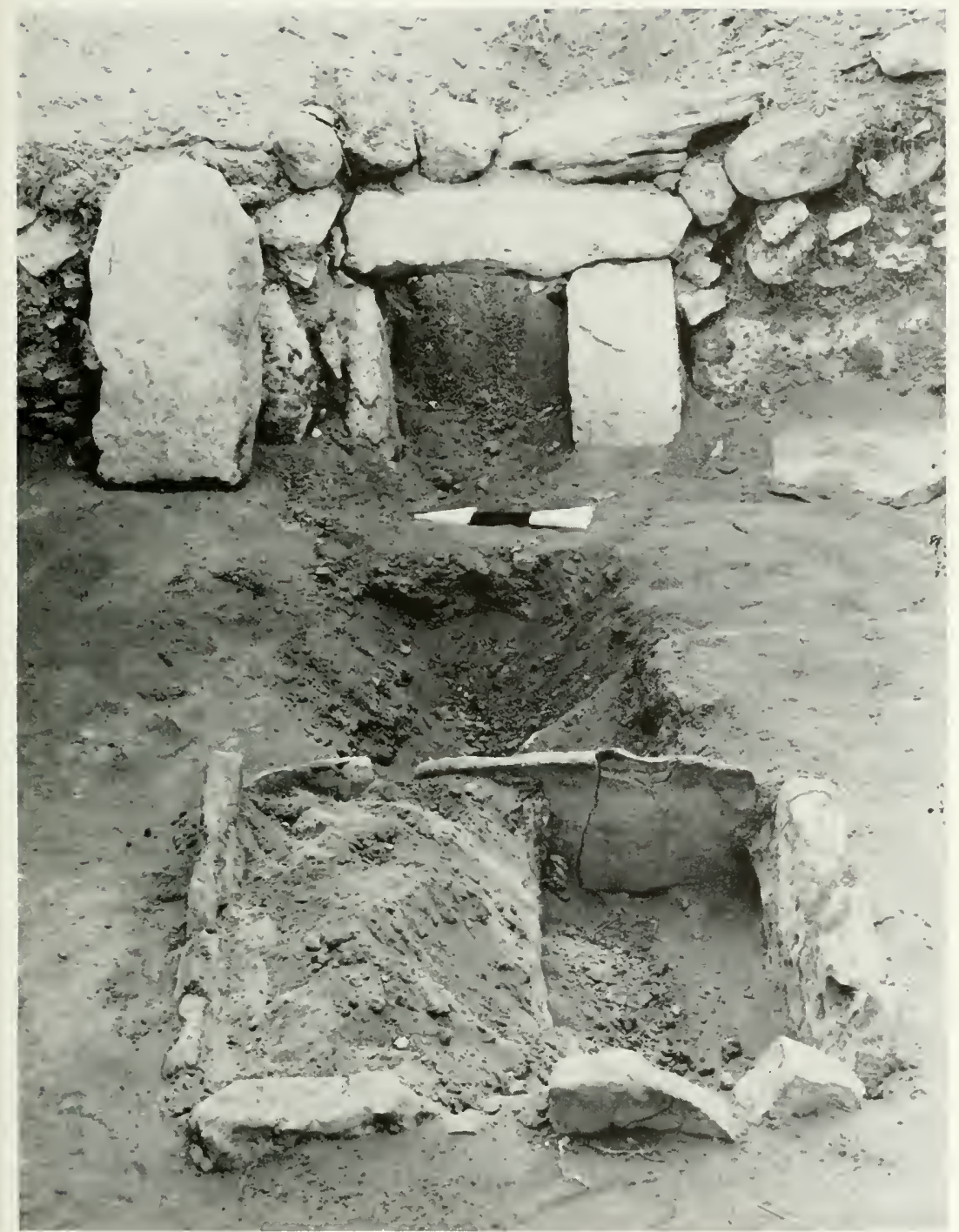

Fıg. 30. Firepit, ladder-pit, ventilator, and damper slab, Room A, Rim Valley Pueblo. Arrow $30 \mathrm{~cm}$. long points magnetic north. 


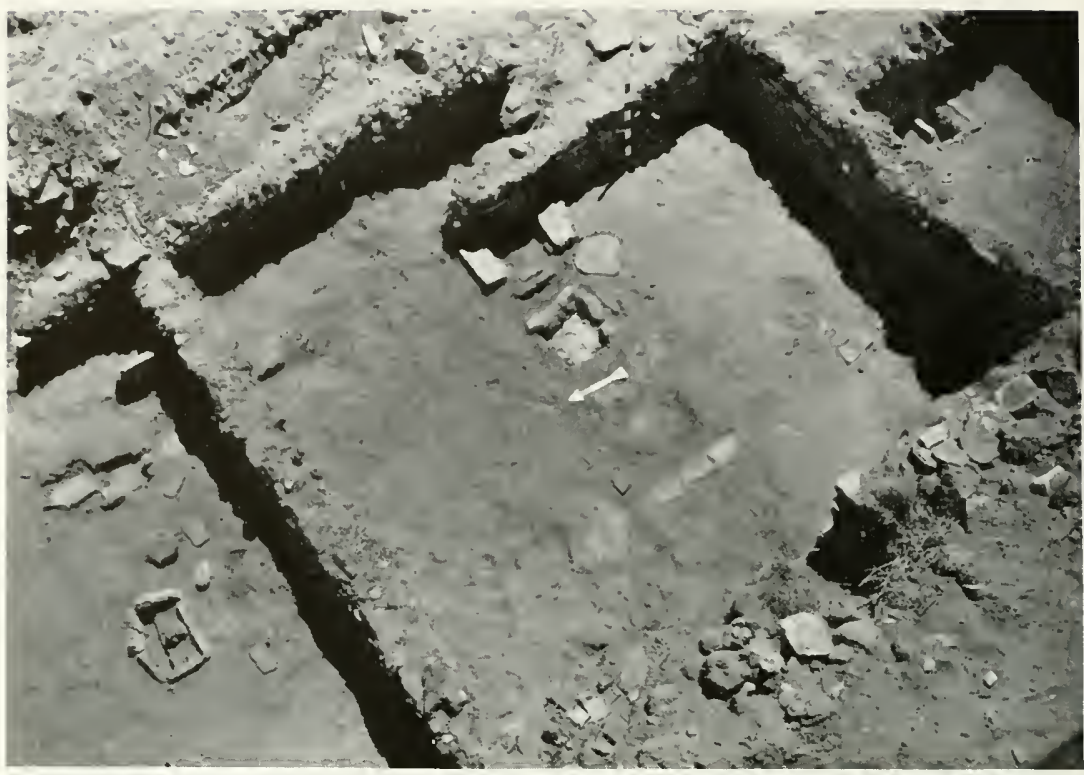

Fig. 31. Room C, Rim Valley Pucblo, with Room B at left and Room H at right. Arrow $50 \mathrm{~cm}$. long points north; meter stick in background.

Ceiling.-Height not known. On the basis of maximum height of walls yet standing, and fallen wall stones it is estimated to have been about 2 meters high. The method of construction is unknown except by inference. Fragments of wooden roof members (beams, poles) cross the shorter dimension of the room.

General Comments.--Rim Valley Pueblo was a small pueblo village consisting of two units both of one story and totaling perhaps twenty-five rooms. The majority of the larger rooms were equipped with firepits, ventilators and other features usually associated with dwelling rooms. A few smaller rooms lacked these features and were probably storage rooms.

The masonry in general seems quite comparable to that of other puellos of roughly the same period and culture (late Reserve Phase, early Tularosa Phase) both in the upper Little Colorado drainage and farther to the south and east. Although the particular type of masonry in which rows of large rocks alternate with several courses of smaller rocks is less neat here than it appears in later ruins, it is consistent enough in construction so that its position seems definitely to be in that tradition of banded masonry which may have been remotely inspired by a style originating in the area of Chaco Canyon. 
Some of the features of Room Ci seem to indicate that it was used for ceremonial as well as secular functions. The association of ventilator, firepit and vault, the elaboration of the ladder-pit(?) with arm-like stone

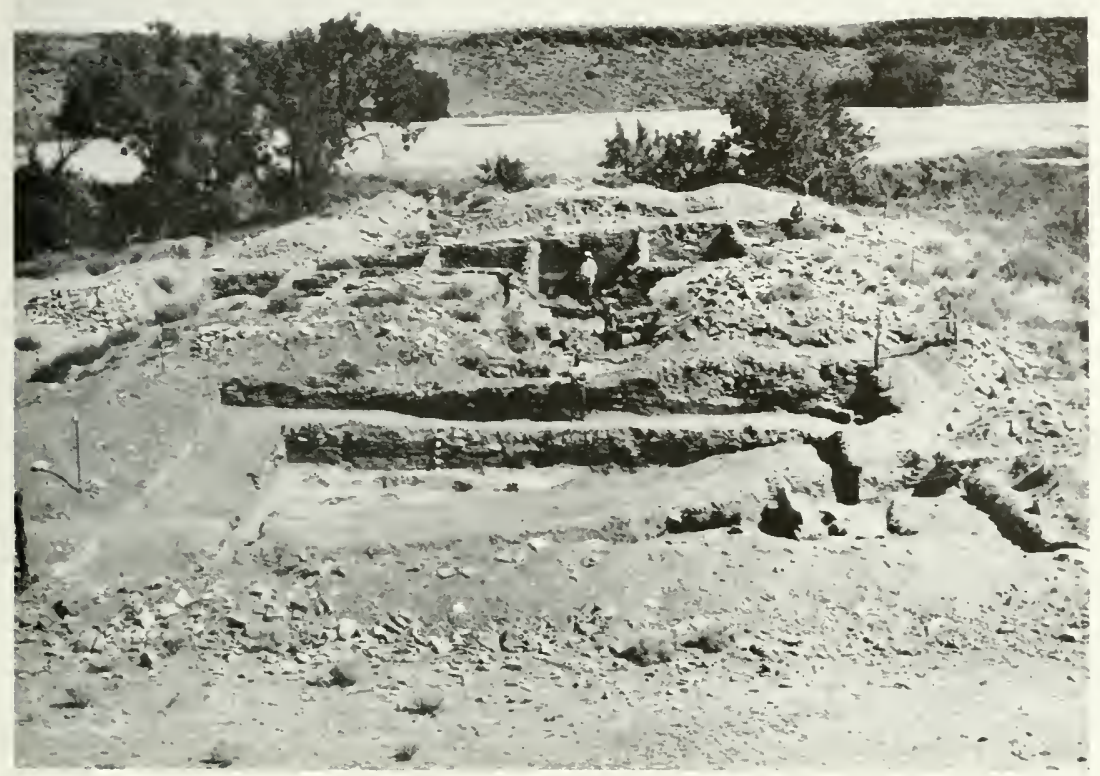

Fig. 32. Hooper Ranch Pueblo, showing Great Kiva in foreground and dwelling rooms in background. Meter stick stands against north wall of Great Kiva.

slabs on each side. and the position of the vault in the normal location in a kiva for a foot-drum type of vault or sipapu seem particularly suggestive. There is also the possibility that the secondary wall through which the ventilator opening passes could have been the face of a platform or bench. However, because it seems a little high for this purpose and was filled behind with very large boulders it seems more probable that this boulderfilled area served as a buttress to strengthen the earlier prinary east wall. These features of Room C when coupled with those from other rooms a possible "kachina" niche in the wall across from the firepit, a ladder-pit and ventilator in Room A (the southwest corner), and evidence for considerable (ritual?) red paint grinding in the southwest corner of Room B across from the firepit and rentilator in that room-seem to hint that at least these rooms were more than ordinary dwelling rooms. Throughout much of the upper Little Colorado drainage and in the Reserve area up to the end of the Tularosa Phase small kivas of the Anasazi type seem to 


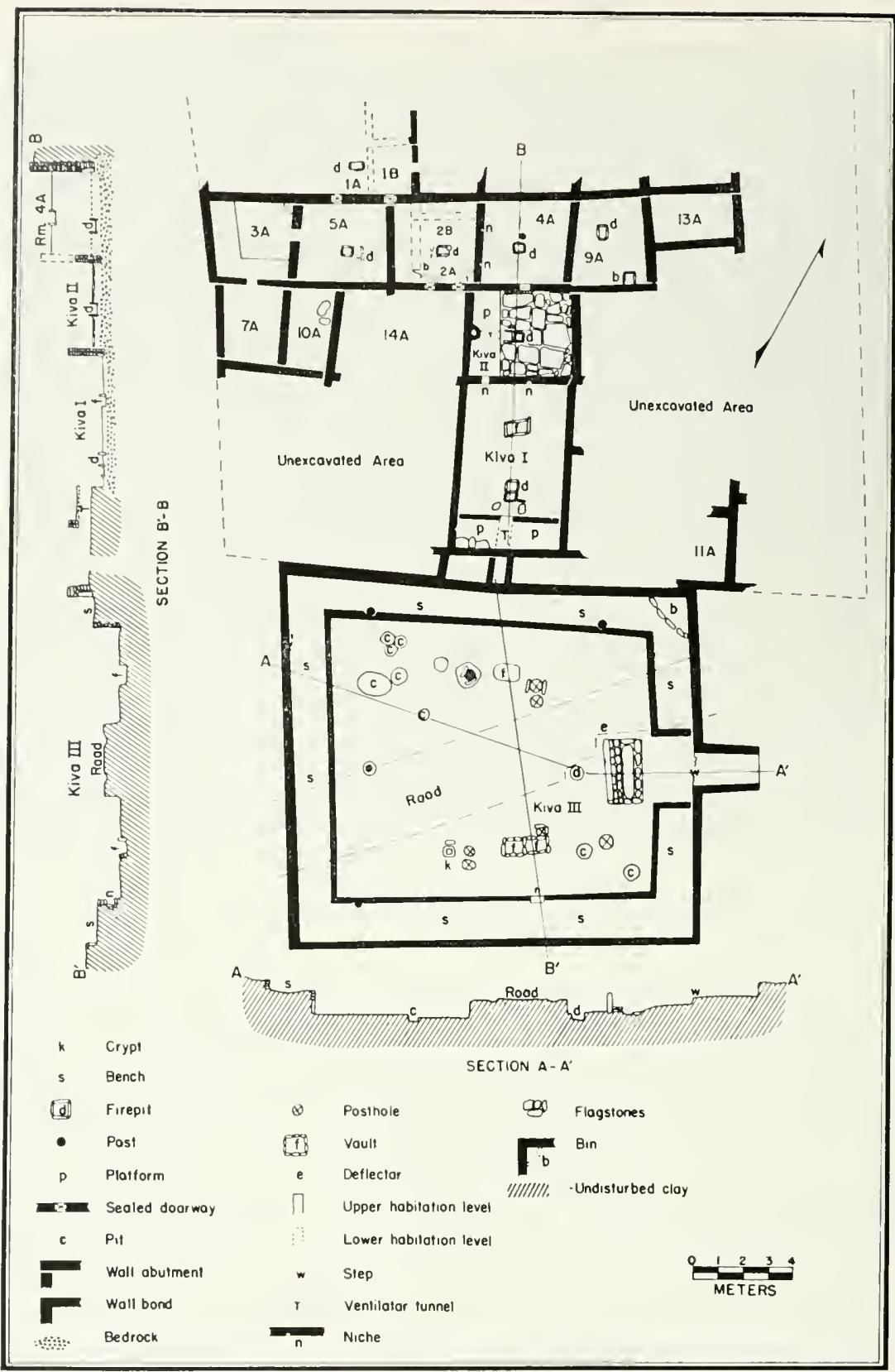

Fig. 33. Plan and sections of Great Kiva and adjacent rooms at Hooper Ranch Pucblo. 


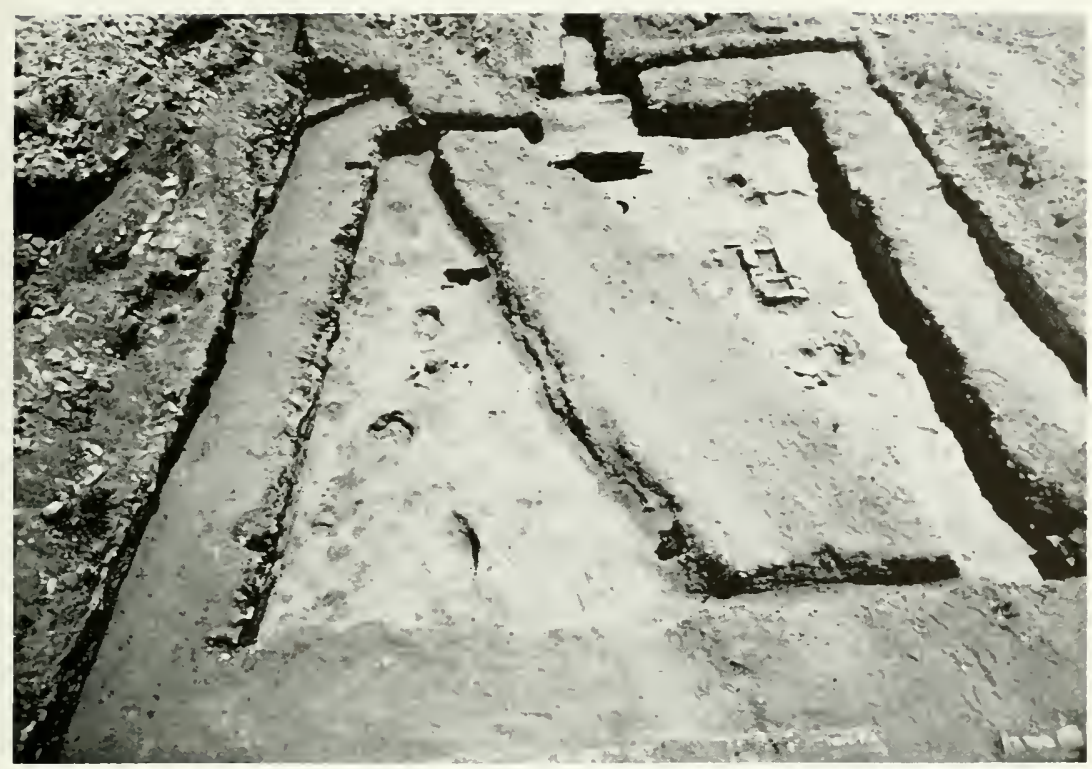

Fig. 34. Great Kiva, Hooper Ranch Pueblo, from the west. Ramp entryway and deflector in background: postholes and vaults in foreground. Access road runs diagonally through kiva. Arrow $50 \mathrm{~cm}$. long at left; meter stick in background.

be lacking. These dwelling rooms with more than the common features possibly could have been used for small group ceremonies as well as for normal dwelling purposes.

\section{THE GREAT KIVA. HOOPER RANCH PUEBLO}

(Figures 32-41)

Hooper Ranch Pueblo is located on the east bank of the Little Colorado River approximately four miles below Springerville (Sec. 8, Twp. 9 N., R. 29 E.. G. and S.R.M.).

The kiva itself is situated on the south end of this ruin, which contained perhaps sixty rooms along with two small kivas of the more conventional Western Pueblo type. It is, moreover, a much larger kiva and is not surrounded by rooms as the others are.

Shape.-Rectangular and fairly symmetrical except that the north wall was constructed to follow the orientation of the rooms adjoining the kiva on the north rather than being parallel to the south wall.

Dimensions. - 15.5 meters east to west and 14.5 meters north to south.

IValls (fig. 35).- Of masonry. Sandstone slabs, some laminated, mostly as quarried or rough hewn, were laid up as a vencer against the 


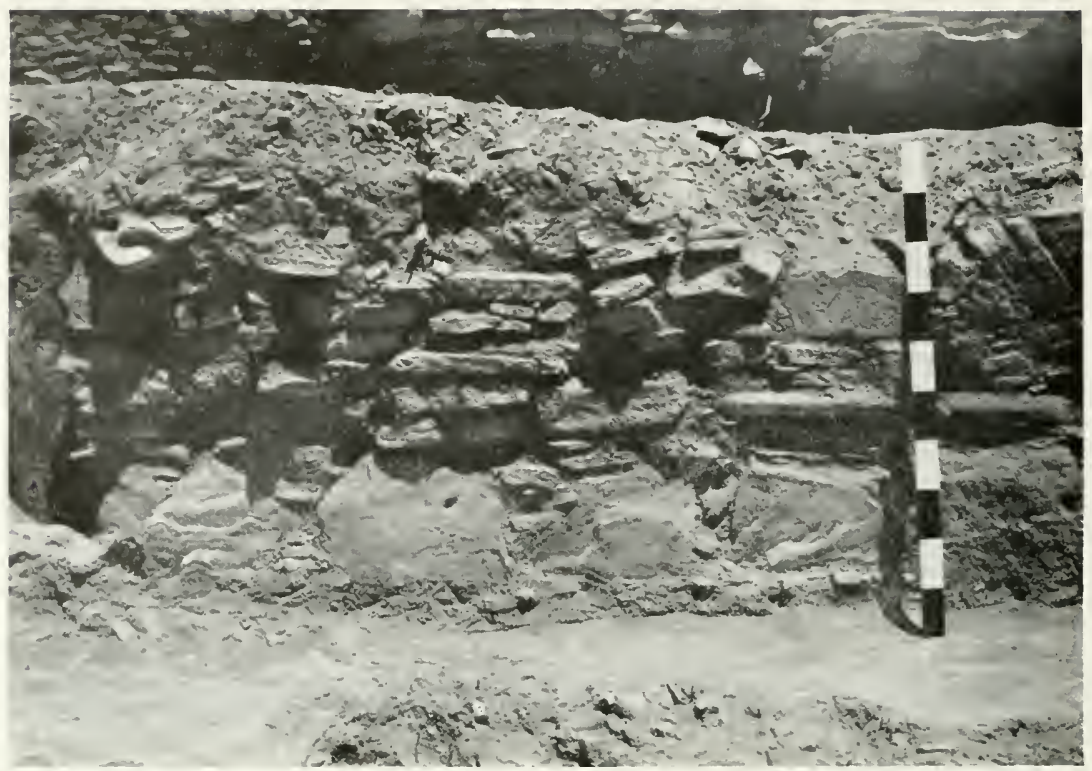

Fig. 35. Detail of masonry in face of bench on north side of Great Kiva, Hooper Ranch Pueblo. Meter stick at right.

wall of the excavation into the native clay or trash. The north wall is composed of two facings, and the stones interlock in the interior; the west wall was built of random rubble: the other walls had large slabs set on edge or end at the base and regularly coursed rubble walls above. The vertical joints between the large vertical slalss were filled with a chinking of stone laid either horizontally or vertically. Thickness: Face of bench. $18 \mathrm{~cm}$.; upper north wall, $45 \mathrm{~cm}$; other walls, $20 \mathrm{~cm}$. A single layer of undecorated, dark gray mud plaster was noted but only on the lower walls.

Pictographs.-Pecked areas were uncovered: (1) on a large vertical slab set in the face of the bench near the middle of the south wall and west of the niche; (2) on a stone at the corner of the ramp entrance.

Viche (fig. 36).--Near the middle of the face of the bench. in the south wall. Rectangular in shape and lined with slabs. Width, $50 \mathrm{~cm}$; height. $28 \mathrm{~cm}$; depth, $28 \mathrm{~cm}$.

Recessed Posts. - One near each of three corners, northwest, southwest and northeast: these were roof support posts. Their lower sections were covered by masonry in the face of the bench.

Floor.-Dark gray adobe clay was applied as a single layer: it was smooth and fairly level. A pit near the northwest corner had been floored over. 


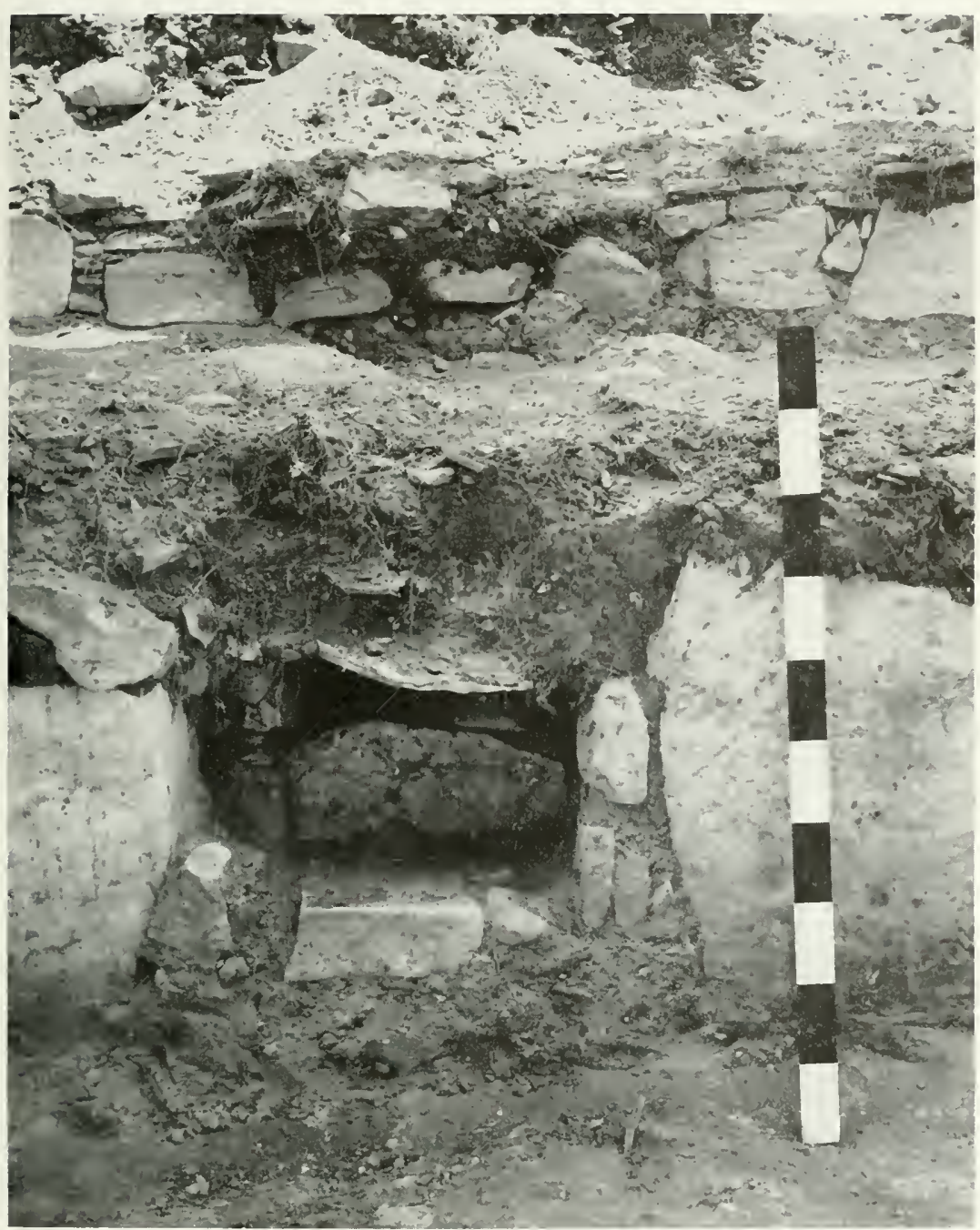

Fig, 36. Detail of niche in face of bench on south side of Great Kiva, Hooper Ranch Pucblo. Meter stick at right.

Bin.-Five large slabs set on edge crossed the northeast corner diagonally on a level with the floor of the bench.

Firepit.-Circular shape, flat bottom; dianeter, $50 \mathrm{~cm}$; depth, $30 \mathrm{~cm}$. It was lined with adobe clay burned red and contained much eharcoal in the form of small fragments, including those of charred corncolss and walnuts. The surrounding floor west of the deflector was burned. 


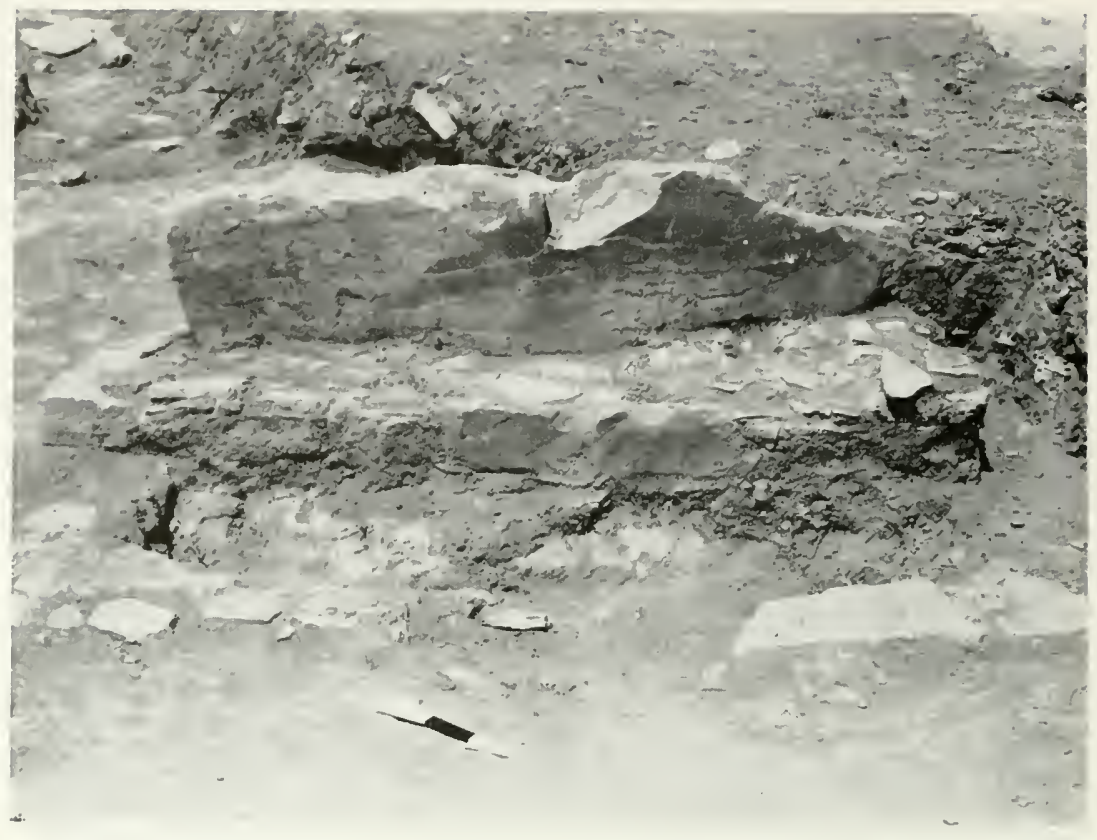

FIG. 37. Deflector, Great Kiva, Hooper Ranch Pueblo, viewed from ramp entryway. Masonry base and masonry-rimmed trough to east. Arrow $30 \mathrm{~cm}$. long points magnetic north

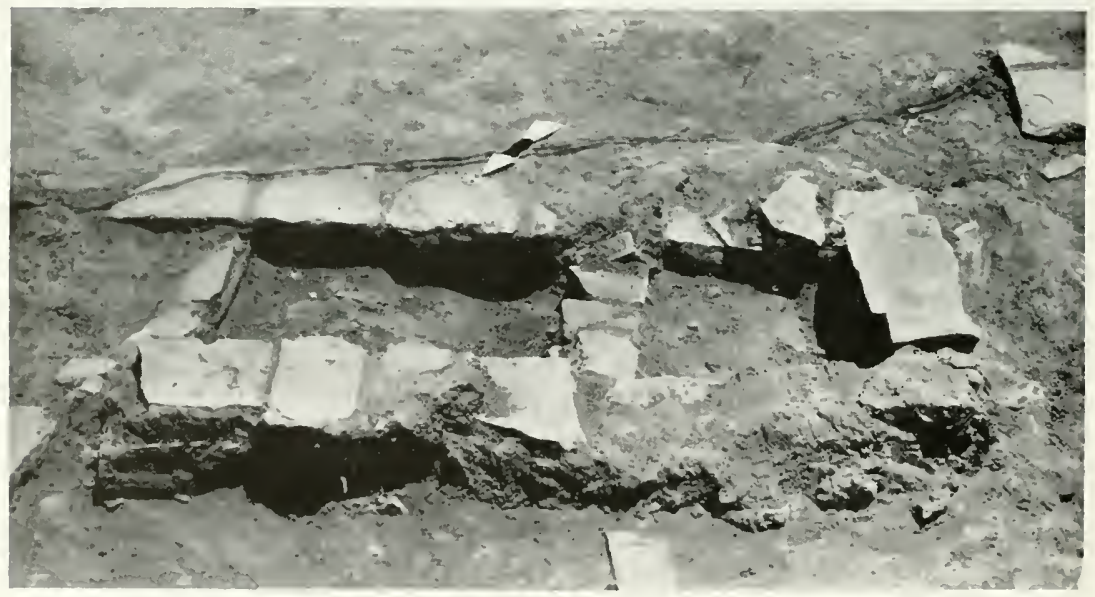

Fig. 38. South vault, Great Kiva, Hooper Ranch Pueblo. . Irrow $30 \mathrm{~cm}$. long points magnetic north. 


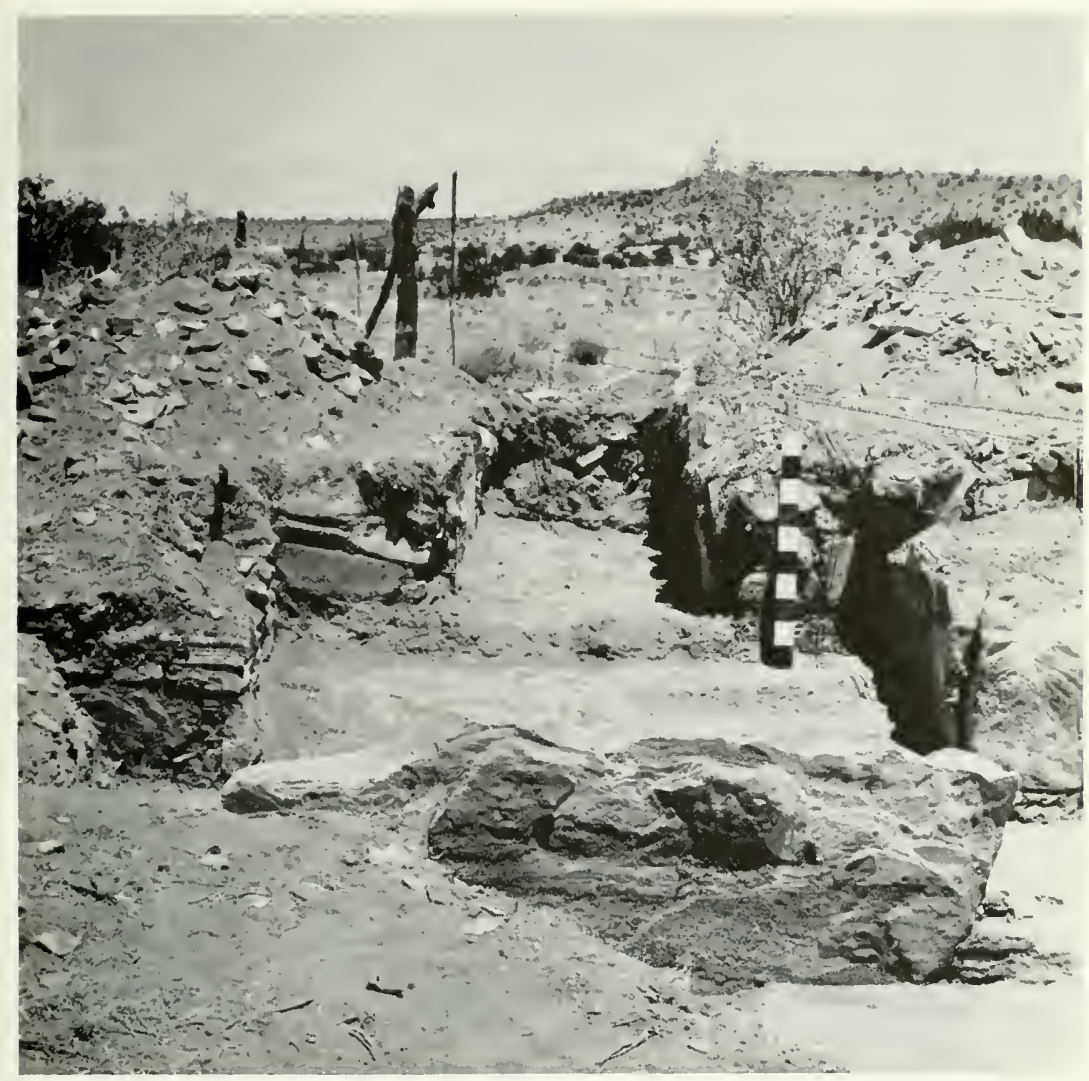

F16. 39. Ramp entryway, Great Kiva, Hooper Ranch Pueblo, showing wider "vestibule" area and narrower portion beyond; huge deflector slab in foreground. Meter stick at right.

Deflector (fig. 37). - A large slab had been set on edge between the firepit and the ramp entryway; it is partially supported by masonry on the east side and at the ends. Length, $240 \mathrm{~cm}$.; height, $72 \mathrm{~cm}$.; thickness, $15 \mathrm{~cm}$.

Vaults (fig. 38).- Rectangular in shape. One is located on each side of the fioor area. The south vault was lined with stone masonry and partitioned into two sections: the north vault was lined with adobe clay and had a floor of stone slabs. Length: south vault. $133 \mathrm{~cm}$. (total): north vault, $85 \mathrm{~cm}$. Depth: south vault, $40 \mathrm{~cm}$; ; north vault, $30 \mathrm{~cm}$.

Crypt (figs. 40, 41). - A square box, with floor and walls of stone slats, has a roof of a ring slab) and a rectangular stone slab for a cover. Length. $38 \mathrm{~cm}$.; width, $36 \mathrm{~cm}$.: depth, $28 \mathrm{~cm}$. It contained a painted sacred stone 
image (see pp. 69-74), a decorated miniature jar. and black and white beads. Some of the beads were found inside the jar, others on the floor of the crypt.

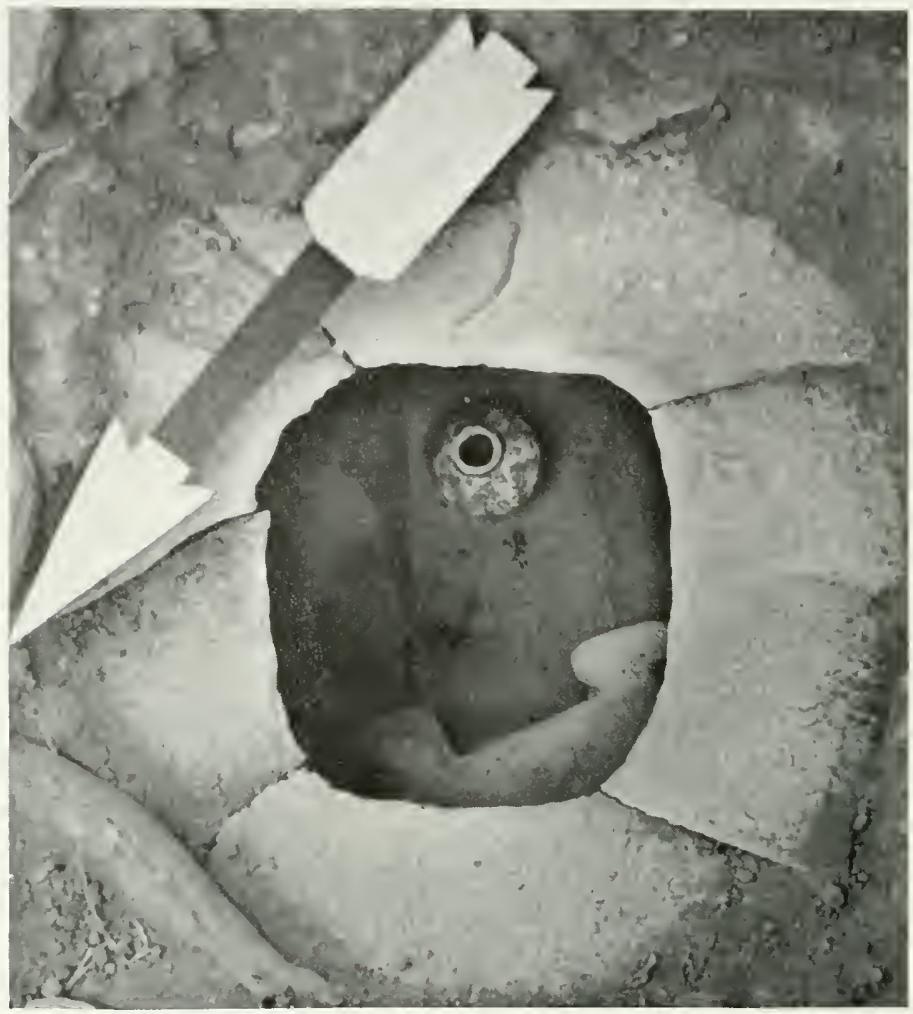

Fig. 40. View through ring slab cover of crypt, Great Kiva, Hooper Ranch Pueblo, showing stone image and miniature jar in situ. Arrow $30 \mathrm{~cm}$. long points magnetic north.

Pits.-One is oblong, wider at one end; and another is circular, with walls and floor of native clay; these walls curve to a basin-shaped bottom. Length of oblong pit, $110 \mathrm{~cm}$; diameter of circular pit, $38 \mathrm{~cm}$.; depths, $50 \mathrm{~cm}$. and $28 \mathrm{~cm}$. Both pits contained many rocks.

Ramp Entryzeay (fig. 39). Oriented to the east, and lined with stone masonry. Its floor slopes up gradually. A low adobe step is in line with the upper east wall of the kiva proper. The entrance widens from 110 to 250 cm. at this point, forming a vestibule between the ends of the bench and the deflector. Width, $100 \mathrm{~cm}$. at outer end; length (outside Kiva proper), $285 \mathrm{~cm}$. 
Posts and Postholes. -Nine principal ones, two of them double. The diameters range from 35 to $100 \mathrm{~cm}$. The posts, 22-30 cm. in diameter, decayed and/or charred, were wedged in with slabs set on edge around

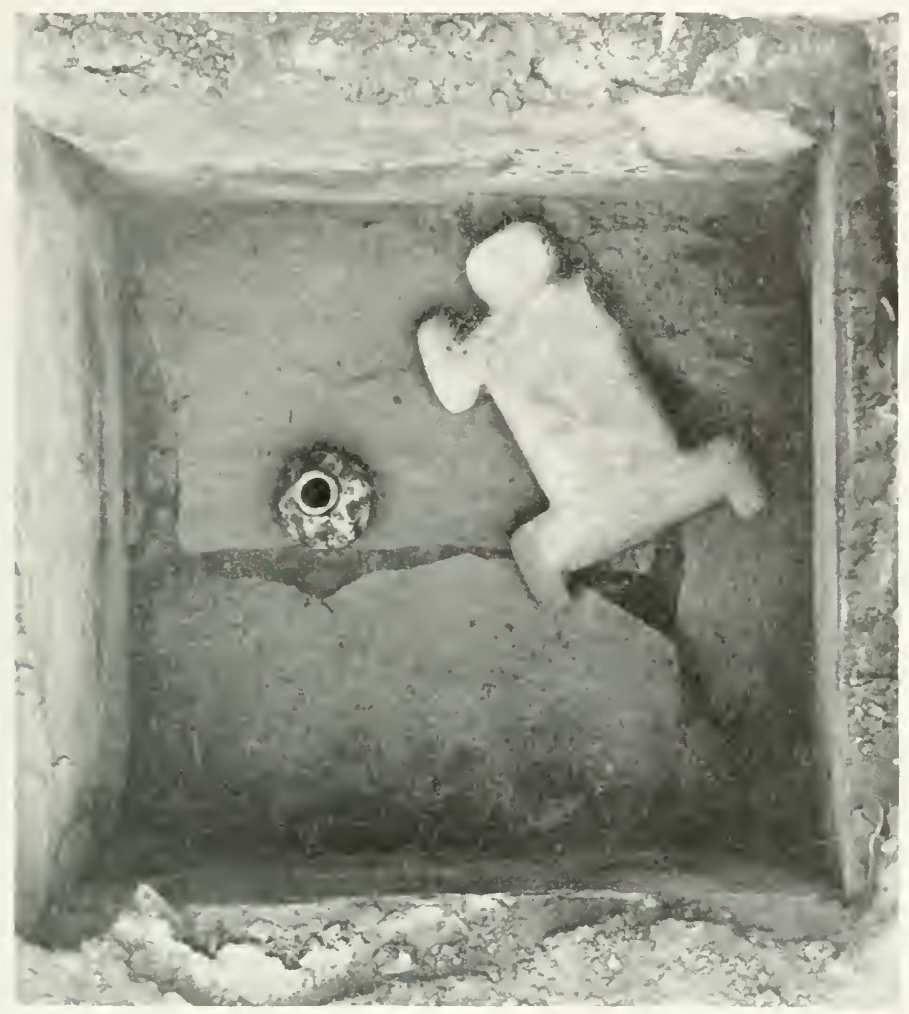

Fig. 41. Crypt in Great Kiva, Hooper Ranch Pueblo, with covers removecl. showing construction detail of interior and objects in position.

them. Depths of the postholes ranged from 60 to $100 \mathrm{~cm}$. Three were located across the west end, two at the east end, and four in a quadrilateral arrangement in the main floor area east and west of the vaults. Two of these postholes were double.

Roof.-Top layers were clay over brush; the exact character of the lower layers is unknown. The alignment of the principal posts and holes suggests that four large beams crossed the shorter dimension of the kiva. The maximum height of the standing upper wall $(130 \mathrm{~cm}$.) added to the maximum height of the veneer masonry for the bench $(85 \mathrm{~cm}$.) indicates a vertical distance from the main floor to the roof of over 2 meters. 
Comparisons. - The rectangular shape of the Great Kiva seems to be most like that of Mogollon Great Kivas such as those described by Hough (1907, pp. 53, 55-57) on the Blue River, and three kivas closer to Reserve, New Mexico: that at the Sawmill Site (Bluhm, 1957, pp. 15-27), a nother at Higgins Flat Pueblo (Martin, Rinaldo, and Barter, 1957, pp. 13-22), and one at Casa Malpais near Springerville (Danson, 1957, pp. 82-83). In this feature, if the ramp entryway is left off it also has obvious relationships to earlier Mogollon small and big kivas (Bluhm, 1957, p. 26) as well as to contemporary and later western pueblo kivas (Smiley, 1952. p. 20).

In size it compares with the Nantack Village Great Kiva (Breternitz, 1959. p. 16) and the other larger kivas mentioned above although it is smaller than the courtyard Great Kiva at Kinishba. On the basis of size - the paramount criterion - it can certainly be classified as a Great Kiva.

The vertical slab masonry is unlike that of any of the other Mogollon Great hivas. It is more nearly comparable to that of Kiva I, Arizona W:10:52, at Point of Pines (Smiley, 1952, p. 40), or Kiva I at Table Rock Pueblo (Martin and Rinaldo, 1960b, p. 158). In this feature it apparently reflects the current style of wall construction rather than a traditional style, although the early Anasazi Great Kivas on Basket Maker III and Pueblo I sites contain somewhat similar masonry; for example, the face of the bench in Kiva I of the Cahone Canyon sites (Martin, 1939, p. 350).

The arrangement of the sub-floor vaults on either side of the Great Kiva has its parallels both in the Mineral Creek Pueblo Great Kiva (Martin, Rinaldo, and Longacre, 1961, p. 26) and in Anasazi Great Kivas on Pueblo III sites (Martin, P. S., 1936, pp. 48-49; Morris, E. H., 1921, p. 119; Roberts, F. H. H., Jr., 1932, pp. 88-89). The use of a small subfloor circular firepit rather than a raised hearth or a large rectangular masonry fireplace has its closest parallels in Anasazi small kivas.

The vaults on the south side of the Great Kivas at the Hooper Ranch Pueblo and Mineral Creek Pueblo were lined with masonry, whereas the north vauts were less elaborate. An analogous situation was observed by Roberts (1932, p. 88) at the Village of the Great Kivas, where the west vault of the Great Kiva was more complex.

\section{SUMMARY OF SECULAR ARCHITEGTURE}

The secular structures excavated during the 1960 season present a hypothetical sequence of architectural development which is complete in itself and yet roughly parallels that of other areas. The first step in this 
sequence is highly conjectural. It is compounded of bits of evidence encountered at Laguna Salada, in some of the pit structures at Tumbleweed Canyon, and from the floor areas at the Chilcott Sites. This postulated earliest type of structure may have been a light brush shelter erected over a compacted floor area, or, on occasion, over a shallow excavation. This stage has its parallel in the Wet Leggett Cochise dwelling area (Martin and Rinaldo, 1950b, p. 430).

Sometimes (as at Tumbleweed Canyon) the nature of the building site necessitated the excavation of rocks to secure a smooth floor and a deeper area more sheltered from the wind. In these instances the rocks were simply piled up around the edge of the excavation. In at least one instance these piles of rocks appear to have been used for the base of a criblike roof structure. This stage has a rough parallel in some of the Pine Lawn Phase sites in the Reserve area such as the Promontory Site (Martin, Rinaldo, and Anters, 1949) where in some rare instances rocks removed in the process of digging a pithouse floor had been piled up around the perimeter. A more definite parallel is evident at the Bluff Site in Houses 6 and 15 of the Hilltop Phase (Haury and Sayles, 1947, pp. 24, 38), which had walls of rubble piled up to hold back the trash out of which they were excavated.

It is conjectural whether these piled-up rocks formed walls that might be typologically and sequentially related to the next development (represented in the Chilcott and Thode sites), which is actual masonry of a crude rubble type. These walls in the Thode and Chilcott sites were built up between adjacent rooms of a series of dwellings clustered together. Although pueblos begin to take form at this stage, there is as yet no preconceived plan. Villages have the form of a series of pithouses or subsurface rooms clustered together in an amorphous group, with only an occasional room tacked on.

Floors were excavated below ground level to various depths and the walls were continued upward above the ground surface of the excavation by means of crude rubble masonry of unshaped field stones and cobbles. In some instances, particularly in the later sites such as Site 31, at Vernon, the earthen wall of the excavation was faced with a veneer of rubble masonry of a single thickness, and continued upward above the ground surface with larger through stones or masonry of a double thickness of stones (Martin and Rinaldo, 1960a, p. 56). Similar developments involving rooms transitional between pithouses and surface houses with crucle masonry walls have been noted in the Reserve Phase both in the Reserve area and in the Point of Pines area (Martin, Rinaldo, and Anters, 
1949, p. 126; Martin and Rinaldo, 1950b, pp. 416-417; Peckham, 1958, pp. 91-93; Breternitz, 1959, pp. 56-57).

The possibility that these crude masonry walls had extensions of jacal construction has been suggested. The quantities of wall stones excavated from these structures in the lernon area favor the conjecture that the masonry walls built there were sufficiently high without a jacal extension for a person to stand upright. No remains of posts have been found inside the walls to suggest more than a minimum of roof support-nothing like the rows of postholes indicating jacal walls that were found at Three Pines Pueblo (Martin and Rinaldo, 1950b, p. 432), and have been postulated for Ruin B at the Nantack Village (Breternitz, 1959, p. 55). This does not mean that jacal walls were not used in the Vernon area, but rather that evidence for their existence has not yet been found.

Although the rooms are spaced separately for the most part, at this stage they tend to approach a rectangular shape, with the exception of those at the Thode Site. There are apparently very few contiguous rooms at sites contemporary with this site (Martin, Rinaldo, and Longacre, 1961). However, in the next phase both Mineral Creek Site and Rim Valley Pueblo are composed of rooms that are contiguous and for the most part rectangular in shape.

The interior furnishings of the earlier houses are very simple and even the later rooms have few. In the earlier houses the milling stones and firepits constitute virtually the only interior furnishings. The firepit is located near one wall or, less often, in a corner. Ordinarily these firepits are simple shallow depressions excavated into the native soil and are not lined with plaster. They are more frequently oval or circular in shape than rectangular. One was outlined with rough stones. Typologically more developed and occurring in later sites are circular firepits which are located near the centers of the pithouses. These are usually somewhat deeper and are lined with adobe plaster.

The later houses, such as those at Site 30 (Martin and Rinaldo, 1960a). are sometimes furnished with storage pits in addition to the milling centers and firepits. A still later development was the construction of rectangular firepits; some of these are plaster-lined, and others are lined on the side with rough stone slabs. These occur in the earlier pueblos such as Chilcott Sites 1 and 3, the Thode Site, and occasionally in the later pueblos such as the Mineral Creek Site and Rim Valley Pueblo. These crude stone fireboxes are frequently associated with ventilators and in at least one instance with a ladder-pit.

The next stage in this development of interior architectural features is found only at the latest sites excavated in the area. It occurs at Table 
Rock Pueblo (Martin and Rinaldo, 1960b), Hooper Ranch Pueblo, and Rim Valley Pueblo, but not at the Mineral Creek Site. This development is represented by centrally located fireboxes with sides and bottoms lined with nicely worked stone slabs. These fireboxes are often associated directly with ashpits or ladder-pits and are set so as to be furnished with fresh air through some form of ventilator. They are also occasionally found in the same rooms with furnishings such as bins and flour receptacles, which have walls made of stone slabs. This trend toward the construction of somewhat more elaborate interior furnishings has its parallels in the later phases, such as the Tularosa Phase, both in the Reserve area (Martin et al., 1956; Martin, Rinaldo and Barter, 1957; Rinaldo, 1959) and at Point of Pines (Wendorf, 1950; Breternitz, 1959; Olson, 1960).

In short, the developments in architecture throughout the upper Little Colorado drainage appear to parallel a similar evolution in the neighboring areas to the south such as the Reserve area and the Point of Pines area. Inasmuch as most of these developments involve some form of stone or masonry construction it seems likely that they had their ultimate source in the Chaco tradition of the Anasazi culture to the north, where these arts reached such a high point. 


\title{
II. Some Convergences and Continuities
}

\author{
By JOIN B. Rinaldo \\ Associate Curator, Department of Anthropology \\ Chicago Natural History Museum
}

\section{THE GREAT KIVA}

The Great Kiva at the Hooper Ranch Pucblo contains a number of elements of construction that appear to be modifications of features found in earlier Great Kivas both in the Mogollon and the Anasazi traditions. So many of the principal features of this kiva, such as shape of floor plan, type of entrance, arrangement of roof supports, and primary orientation, are Mogollon in derivation that the character of the entire structure is of a distinctly Mogollon cast. Yet some of its furnishings, such as a deflector, a particular type of vault, a masonry-faced bench, and a wall niche, seem to indicate that the builders must have been at least influenced in their planning by the Anasazi tradition. Finally, it contains a few featuresa type of bench, a form of crypt, and an arrangement of roof beamswhich strongly suggest parallel features in historic Western Pueblo kivas.

The rectangular shape of the floor plan (fig. 33) is almost certainly of Mogollon derivation. Many of the earlier Mogollon ceremonial structures from the Circle Prairie Phase on up through the Three Circle and Nantack Phases are rectangular (Wheat, 1954, p. 62; Haury, 1936a, p. 62; Martin and Rinaldo, 1950a, p. 284; Breternitz, 1959, p. 18; Bluhm, 1957, p. 15). Moreover, almost all of the later Mogollon Great Kivas are rectangular (Bluhm, loc. cit.; Olson, 1960, p. 199: Martin, Rinaldo, and Barter, 1957, p. 13). Some of the exceptions, such as the earlier structure at Higgins Flat Puelolo (Martin, Rinaldo, and Barter, loc. cit.) and the Mineral Creek Site (Martin, Rinaldo, and Longacre, 1961, p. 23), may represent examples of either cultural lag or stronger Anasazi influence.

The entrance (fig. 39) was of the ramp type with a short step as one entered the "vestibule." This type of entrance is considered typical of Mogollon Great Kivas. It is generally similar to those excavated in the Reserve area (Martin, Rinaldo, and Barter, 1957, p. 18; Bluhm, loc. cit.; Rowe, 1947) and to those described for the Blue River area (Hough, 1907, pp. 53, 55-57) and for Point of Pines (Breternitz, 1959, p. 17; Olson, 1960, 
p. 192). It is both shorter and narrower than most of these, although it is almost as wide where it widens out into the "vestibule." In common with the entrance of the Great Kiva at the Dry Prong Site it has a step, as do the entrances of several other Great Kivas (Nantack Village. Sawmill Site, Higgins Flat Pueblo). Somewhat similar lateral entryways with steps have been found in earlier Mogollon pithouses (Haury, 1936a, figs. 6, 21-23; Martin and Rinaldo, 1950a, p. 276), and the concept may be derived from these or ultimately from the Hohokam (Gladwin, Haury, and Sayles, 1937, p. 61).

The use of roof support posts in groups of three pillars in some rows and two in others also seems to have parallels in the earlier Mogollon Great Kivas. This arrangement is most clearly seen in the later Great Kiva structure at the Sawmill Site (Bluhm, 1957, fig. 3). A similar grouping may be separated out at Higgins Flat Great Kiva (Early) if rows of posts oriented parallel to the front and rear walls are selected from the pattern (Martin, Rinaldo, and Barter, 1957, fig. 2: Olson, 1960, fig. 6). Furthermore, the plaza at Foote Canyon Pueblo (which may have funetioned as a Great Kiva) presents a similar arrangement of large posts (Rinaldo, 1959, fig. 66).

In this connection the positions of the posts and the distance between them suggest that in the Hooper Ranch Great Kiva sets of beams or girders crossed the kiva at right angles to the ramp entrance and across the shorter dimension of the kiva, possibly with two beams side by side across the middle section, where there are double postholes. However, we did not find enough of the roof structure to know whether the main beams actually followed this orientation and positioning - as was the usage in historic lesser kivas such as that at Shipaulovi (Mindeleff, 1891, fig. 23) or at Hawikuh (Hodge, 1939, fig. 3) and therefore was an innovation when this Great Kiva was built-or instead followed some other arrangement in continuation of the customs in earlier Great Kivas such as that at the Dry Prong Site (Olson, 1960, p. 192).

The primary orientation of the entire Great Kiva, with its long axis through the hearth and firepit area, the deflector, and the lateral entrance running from west to east, is another Mogollon characteristic. This general orientation is typical of Mogollon pithouses and Great Kivas as contrasted with those of the Anasazi, which are generally oriented north to south.

Deflectors (fig. 37), even of the most rudimentary sort, are relatively rare in Mogollon structures, although they do occur at the Harris Village (Haury, 1936a, fig. 22) and at Turkey Foot Ridge (Martin and Rinaldo. 1950a, p. 389). As they are a customary furnishing of Anasazi lesser kivas 
and pithouses and are found even in Chaco Great Kivas (Vivian and Reiter, 1960, p. 90) it would appear that the huge slab set in a masonry base between the entrance and the hearth area in the Hooper Ranch Pueblo Great Kiva is a feature that probably was derived from the Anasazi tradition.

The benches found in Anasazi Great Kivas have been discussed by Vivian and Reiter (1960, p. 88). Like these, the bench (fig. 34) of the Hooper Ranch Great Kiva is faced with masonry, is relatively level, and surrounds a lower floor area. Although the concept of a bench, its location (surrounding a main floor area), and its masonry facing seem to be derived from the Anasazi tradition, in dimensions and certain details of construction the bench in this latter Great Kiva is more like the bench in the Great Kiva at the Dry Prong Site (Olson, 1960, p. 190), which this Great Kiva resembles in other features as well. This bench is both higher and wider than the benches in Anasazi Great Kivas (Morris, 1921, p. 115; Martin, 1936, p. 50; Roberts, 1932, pp. 91-92; Vivian and Reiter, 1960 , pp. 12, 29, 39, 44, 56, 63, 67), and the masonry veneer covers a native gravelly clay rather than a rubble core.

The benches in these wo Mogollon Great Kivas appear to have parallels in the banquettes of lesser Hopi kivas both at Awatovi (Smith, W., 1952b, pp. 5-6) and at the other Hopi towns (Mindeleff, 1891, pp. 122129). In fact, the width and general arrangement of the bench in the Hooper Ranch Great Kiva suggest that this area may have been used by spectators and participants who sat or stood, as they do in the Hopi kivas, on the banquette and the elevated portion or platform (Voth, 1901, pp. 92-93).

The rectangular slab-lined niche (fig. 36) in the center of the face of the south bench would seem to be another example of a feature in the Anasazi tradition. Niches, or, as Vivian calls them, "wall crypts," are generally lacking in Mogollon pithouse-kivas and Great Kivas, but they have been found relatively often in Anasazi lesser kivas and Great Kivas ( Vivian and Reiter, 1960, p. 84).

Also suggestive of Anasazi inspiration is the fact that the niche and the north and south vaults form a southward oriented row or secondary axis of features across the short dimension and through the center of the Great Kiva. This "axis" seems to link up directly with the row of features in Kiva I (ventilator, ashpit, firepit, vault and kachina-kihu) with which it is roughly in line (fig. 33, section $\mathrm{B}-\mathrm{B}^{\prime}$ ). The north to south orientation of this row is the traditional arrangement found in the majority of kivas in the Zuni, Acoma and Hopi villages (Mindeleff, 1891, pp. 115, 116) and in most Anasazi pithouses and kivas (Kidder, 1958, 
p. 246), whereas generally in the earlier Mogollon culture an eastward orientation prevails. The niche seems to constitute the focus for the secondary north to south axis in the same way that the lateral entrance does for the primary eastward orientation.

The north vault, which was not lined with masonry, seems to be analogous to the simple resonator pits found in some earlier Mogollon Great Kivas (Bluhm, 1957, p. 18; Olson, 1960, p. 193; Martin, Rinaldo and Longacre, 1961, pp. 29, 58,59). But the south vault (fig. 38), which had masonry lining, division into two compartments, and general complexity, and was also contiguous to a primary roof support posthole. resembles the vaults in Anasazi Great Kivas to a considerable degree. The greater complexity of the western vaults in Anasazi Great Kivas has been noted by Roberts (1932, p. 88) and Vivian and Reiter (1960, p. 93). At Hooper Ranch and at Mineral Creek Site the analogous southern vaults were the more complex, and they included the use of stone masonry, a feature which does not appear in the grooves and resonators of the earlier Mogollon Great Kivas to the south, and which is probably derived from the Anasazi. It is probably no mere coincidence that the crypt containing the sacred stone image was in this area of the Great Kiva floor and was associated with this vault.

It is interesting to observe that this crypt (fig. 40) had a double cover, the lower part consisting of a perforated slab or ring slab, the upper, a rectangular worked slab. This appears to be another instance in which the perforated slab for a small structure such as a niche formed a frame similar to that used for the doors or hatchways of dwellings, as at Kintiel (Mindeleff. 1891, pp. 192-194), at Four Mile Ruin (Fewkes, 1904, pp. 160-161), and at Table Rock Pueblo (Martin and Rinaldo, 1960b, pp. 157, 174). Once again, as in the kachina-kihu at Table Rock Pueblo, the concept seems to be that of a spirit's entrance, but in this case the idea is reinforced by the occurrence of the stone image within the crypt.

According to Stephen (Parsons, Editor, 1936, p. 261) there was a niche-cache (his term) which contained an image in the Wikwalobi kiva at Sichomovi. This he records as follows: "Tihkuyiki (Childbirth water house) or Tuwabontumsiki, the phallic niche-cache in this kiva ... is an oblong rectangle, say six by eight inches. It contains one object, an image. The cavity is about fifteen inches deep. Tuwabontumsi, Sand altar woman, is the wife of Masauwu, and the sister of Muriyinwu. She gave birth to all kachina. She is also called Muriyinmana, also called Tihkuyi, Childbirth water." Stephen does not say whether this "nichecache" is in the floor, the bench or the wall, but there is an obvious resemblance in shape and function. 
There was no direct evidence as to the use of the vaults in the Hooper Ranch Great Kiva. As neither of them showed signs of fire or contained ashes, they were certainly not auxiliary firepits. Although they could have been used as foot-drums there is no evidence for it other than the parallels mentioned above, and they are too small for use as sudatories, as Vivian and Reiter have suggested (1960, p. 93). The division of the south vault into two compartments has its parallel in Great Kiva I at Pueblo Bonito (op. cit.. p. 67) and this seems to lend credence to another suggestion Vivian and Reiter have made concerning their use as containers for growing beans, corn or other plants as part of a hypothetical ceremony possibly ancestral to Powamu. It is suggested that one compartment could have been used for growing beans and the other for growing either beans or corn.

However, if such were the case, the diminutive size of the firepit (diameter, $50 \mathrm{~cm}$.: depth, $30 \mathrm{~cm}$.) relative to the air space to be heated (estimated at over 500 cubic meters) within the Great Kiva presents a problem, assuming of course that the postulated ancestral Powamu ceremony was much like the contemporary ceremony in which plants are forced during the coldest month of the year. The huge size of the fire screen provides a possible answer. When excavated, the firepit contained mostly charcoal, but the area surrounding the firepit for some distance was burned red. This suggests the possibility that the burned area was used for a hearth, as in the earlier Great Kivas at the Dry Prong Site, the Sawmill Sitc and Higgins Flat Pueblo, and that the small circular firepit was used primarily for the storing of hot coals, as in the firepits at Site 481 in the Quemado area (Smith. W., 1950, p. 396).

On the whole, the Hooper Ranch Great Kiva seems to constitute an example of converging traditions, formed as it is of architectural features stemming from both the Anasazi and the Mogollon ceremonial structures. The deflector, the masonry lining and division into compartments of the south vault, the masonry veneer, and the general concept of the bench and the wall niche are parallel to features which have been found more frequently in Anasazi lesser kivas and Great Kivas. But the rectangular shape of the floor plan, the lateral stepped entrance, the general arrangement of the roof supports, and the primary orientation of the structure toward the east are elements represented more strongly in Mogollon pithouses and Great Kivas. Thus the Great Kiva contains within it the elements of a convergence of the Chaco tradition of the Anasazi and the Tularosa tradition of the Mogollon, a convergence which is more clearly exemplified in the ceramics which the kivas contained. 


\section{THE SACRED STONE IMAGE}

Before we discuss this stone figure a word of caution is perhaps needed. In our attempt to probe the relationships of the stone image to possible present-day counterparts we have tended to emphasize Hopi rather than Zuni similarities. This is not because the culture at Hooper Ranch Pueblo appears to be more closely related to Hopi culture. Quite to the contrary, we feel that the architectural and ceramic traditions, the settlement patterns and other traits proside a more definite link with the Zuni culture. However, the literature on the Hopi is in general more complete, particularly on those subjects with which we are concerned here, so that it is much easier to draw parallels in the direction of Hopi culture. Of course, there are also other traits, both in ceramics and architecture, which might link this culture in that direction, but they tend to be represented to a lesser extent than the Zuni traits.

The sacred stone image (fig. 42) is sufficiently specialized in form and decoration to enable us to examine it with more assurance than was possible with the similar figures that appear in the pictographs on the walls of the pueblo rooms (Martin, Rinaldo and Longacre, 1961, pp. 55-56). The posture of the arms and legs is similar to that of the pictographs, but the sculpturing of the hands and feet of the image was done with greater precision, and the features of the face and the decoration in colors which are found on the effigy do not appear at all in the pictographs.

This is clearly an anthropomorphic figure. The left arm is upraised and bent at the elbow. The right arm is missing. broken off in ancient times. The legs are spread out and bent at the knees. The nose. chin, hands and feet are carved in relief. and the mouth and possible vulva appear as small cavities. The hair and eyes are painted black, and the left cye is lower than the right and roughly diamond-shaped rather than osal. The front of the body, the face, and the limbs are painted yellow. The hands are black, bordered by a red stripe at the wrist. and the feet are bordered by red stripes and possibly by black stripes(?) at the ankles. The body is decorated on the front by a series of vertical stripes in the following sequence (proceeding from the figure's left side) : yellow, bluegreen, red, black. yellow, blue-green, black (center stripe). blue-green, yellow, black, red.

Except for white (east), which is omitted, these are the directional colors of both Hopi and Zuni (Parsons, 1939. pp. 186, 218, 172; Bunzel, 1932. pp. 670, 714; Voth. 1901. p. 75). It may be pure chance that the first few color-directions of this series happen to be in the same sequence that the Hopi use in their ritual circuit-yellow (north), ${ }^{1}$ blue-green

1 Actually northwest, southwest, southeast, etc. points of sunrise and sunset at the solstices. 


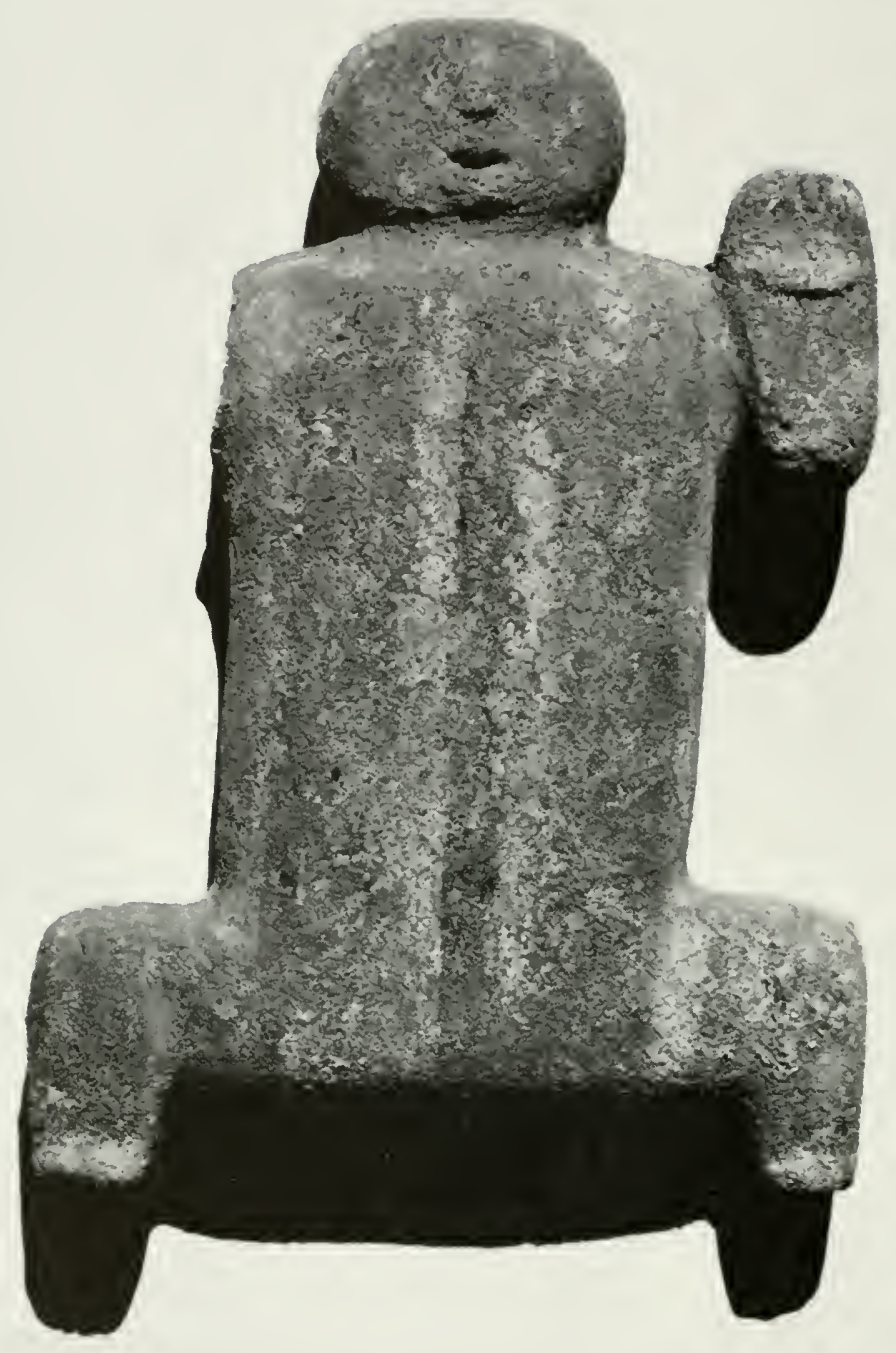

FIG. 42. Painted sacred stone image, Great Kiva, Hooper Ranch Pueblo. 
(west), red (south), black (zenith), but omitting white (east). The factor which suggests that this particular sequence might be Hopi rather than Zuni is that among the Hopi the color for zenith is black, whereas among the Zuni it is "all colors" or "speckled" (Parsons, op. cit., pp. 172, 365; Voth. loc. cit.; Bunzel. loc, cit.). Zenith is always named after east and before nadir by both Zuni and Hopi. Although the series in the directional circuit is the sequence followed in the colors of some ceremonial objects and paintings (Voth, 1901, pls. 42, 47, 53; Stevenson, 1904, pls. 74, 108) it is not used on the majority; so this clue as to the cultural identity of the image is weak.

However, there are some additional clues which seem to corroborate this color-directional symbolism among the former occupants of Hooper Ranch Pueblo. (1) The figure was accompanied in the crypt by a miniature narrow-mouth jar containing six black beads, five white beads, one blue-green bead, and a red chip of stone. These objects might be interpreted to represent the directions zenith (or nadir), east, west and south respectively (omitting north). (2) Yellow pigment in quantity and yellow bone beads were found in the north vault of Kiva I. (3) Painted stones on which green was the dominant color were found near the center of the west wall of the Great Kiva.

Aside from the obvious clues to its religious character provided by the position of the stone image in a large ceremonial structure, the associated sacred objects in the crypt and the lavish use of the directional colors suggest that this represents a supernatural being. But the question arises as to what kind of a supernatural being - a proto-kachina, a cult deity, or a clan wuya? The wuya (a clan protector, clan symbol, or clan ancient) finds so little expression in the literature (Titiev, 1944. p. 155) that we found nothing specific to tie to and felt at a loss to pursue the matter further.

A search was made through the literature pertaining to kachinas and through our museun collections for a figure closely resembling this image but we were unable to find any. Except that one eye is lower than the other and of different shape (cf. Smith, W., 1952b, p. 123, note 45) and there is possibly a straight throat line with a black band underneath (which creates the impression of a half-mask) there are no facial features of the image suggestive of a mask. The nose and mouth are generally naturalistic in form and there is no evidence of headdresses, horns, feathers, beaks, tubular mouths, "beards," special face painting, or other attributes ordinarily found on kachina figures (Colton, 1959; Fewkes, 1903). Furthermore, there were no kachinas in the same posture as the imagearms raised and elbows bent, legs spread out and knees bent, etc. However, some were found which were similar in one or two details. For 
example, a few have yellow masks with black eyes (Citulilu, Fewkes, 1903, pl. 44), but are unlike the image in other details. Vertical stripes on the body are seen on Patun or Squash (op. cit., pl. 52, p. 116) and on Rainbow (Stevenson, 1904, pl. 74). They also appear on a number of dolls of the older flat type in our collections, but on these the stripes are almost always in fewer colors.

There is a closer degree of likeness between the stone image and the figurines of cult deities which appear on the altars in the Marau and Wuwutcim ceremonies of the Hopi. This seems to be particularly true of versions of Talatumsi and Marau-mana (Parsons, Editor, 1936, pp. 883, 964, figs. 467, 484, pl. 23; Fewkes and Stephen, 1892, p. 196, pl. 1, fig. 2; Fewkes, 1894, p. 69; Voth, 1912, pls. 5, 10,13). These are usually represented with flat yellow faces, black hair and eyes, and yellow torsos of roughly the same proportions as those of the image. On at least two of the figurines the feet are rendered in some detail and have flat soles like those of the figurine, so that they can be stood alone on the altar floor or tied to the "rainbow" bar. Their feet are usually spread apart a small distance and they have their arms upraised in a pose similar to that of the image. Talatumsi is clad in cotton garments on occasion and there is the possibility that the stone image was similarly clothed at one time (Parsons, Editor, 1936, p. 964), so that the vertical stripes or body-painting may be irrelevant to identification.

The posture with arms upraised and feet spread apart with knees bent is occasionally seen in depictions of other cult deities, Alosaka, for example (Fewkes, 1903, pl. 59), and there is a striking resemblance in several attributes to the figures of anthropomorphic supernatural beings seen in some Navaho sand paintings. The somewhat elongated form of the torso, the position of the arms and legs, the longitudinal multi-colored stripes on the body and the yellow face are found on these figures in some instances (Wyman, 1952, fig. 38, for example). Because these sand paintings are thought to have retained some of the archaic features of the older Pueblo dry paintings (from which they were derived) these Navaho figures may indirectly corroborate our identification of the image as a representation of a supernatural being, possibly a cult deity. It is therefore suggested that in particular the posture is symbolic of cult deities as differentiated from kachinas although it could equally well be a conventionalized attitude representing childbirth, sexual intercourse (Cosgrove, 1932, pl.225, f; Smith, W., 1952b, figs. 53, $b, 92, a$ ) or any one of several other alternatives.

There is further evidence for the female character of the stone image in its dominant yellow color, which among contemporary Pueblo Indians is symbolic of females (Parsons, 1939, pp. 102, 275) and there is also some 
archaeological confirmation for yellow being a "female" color. Six figures in the Awatovi murals and one in a Mimbres Polychrome bowl -all definitely female - are painted yellow (Smith. W., 1952b, figs. 51, c, 53, b, 67, $d, 78, a, b, 81, b$ : Nesbitt, 1931, pl. 23, b). Moreover, they are similar in other attributes. Five of the Awatovi figures have upraised yellow arms (Smith, W., 1952b, figs. 51, c, 53, b, 78, b, 81, b) and two of them have black hands like the stone figure (op. cit., figs. 53, b, 81, b). There are other archaeological parallels; the similarity in posture between that of the stone image and those of the anthropomorphic figures in certain Four Mile Polychrome bowls is inescapable (Martin and Willis, 1940, pl. I; Fewkes, 1904, pl. 25, a); and, as mentioned above, the posture is much like that of the anthropomorphic pictographs found on the walls of the dwelling rooms at the Hooper Ranch Pueblo. A figure in this posture was also found painted on a stone slab at Kinishba (Cummings, 1940 , pl. 34).

Taken together, the data favor the identification of the image as a female cult deity related to the underworld rather than as a protokachina. The resemblances of the image to cult deity figurines of the present-day Hopi are particularly important in this connection and have been discussed in detail. The location of the image in a crypt in the Great Kiva floor bears out the relationship to the underworld. The crypt is analogous (on a small scale) to the kiva itself, with the aperture apparently representing the kiva entrance. The aperture is also probably symbolic of the entrance to the underworld, and the crypt of the underworld itself. As such it must have been a particularly sacred place. The location of the image in this crypt seems to indicate that the deity represented by the image was related to the underworld. In Stephen's time the figure of Tuwabontumsi (a cult deity) was kept in a "nichecache" in the kiva (see p. 67), and while today the figure of Talatumsi (another female cult deity) is kept in a shrine on the cliffs (Titiev, 1944, p. 131, pl. 3, b), this may be a relatively recent custom.

More specifically, the image may be identified as representing a female cult deity belonging to a group that is concerned with childbirth, reproduction and fertility (particularly fecundity in men and animals rather than in vegetation). Included in this group are those female deities mentioned above: Talatumsi, Tuwapongtumsi and Marau-mana. These may have been differentiated from a single ancestral deity.

There is a possibility that the image represented the ancestral deity from which the three (or more) present-day deities (Talatumsi, Tuwapongtumsi and Marau-mana) were differentiated long ago. There is also the possibility that it represented simply another deity, also of the 
same group, who has been forgotten; and no doubt there are other possibilities. Most of the data secm to support the theory that the image represents a cult deity rather than a proto-kachina, although we cannot rule out that possibility either, for the asymmetrical eyes and the lower face have mask-like qualities, and the vertical striping resembles that of some of the older kachina dolls. However, resemblances in hands, feet, head, general torso form and posture are closer to those of both ancient and modern representations of cult deities. Its position in the Great Kiva crypt - a particularly sacred place - bears this out and also its relationship to the underworld. Its sexual parts and dominant yellow color indicate that it is a female. As might be expected, it is most like the older representations of supernatural beings, particularly those yellow central figures in the Awatovi murals who stand with their arms upraised as if bestowing blessings.

Dr. Fred Eggan (verbal communication) has suggested that this differentiation may have occurred through the addition of new population groups with similar beliefs and rituals, with subsequent partial equating of the deities involved, or through later development of parallel cults consequent on population increase, and with different versions of cult deities and rituals. 


\title{
III. Pottery
}

\author{
By Paul S. Martix \\ Chief Curator. Department of Anthropology \\ Chicago . Vatural History Museum
}

\section{GENERAL REMARKS}

The study of prehistoric pottery from a given area is a vast undertaking and one could devote an infinite amount of time to it. Not only does one have the materials of manufacture-clays. tempering materials. slip-clay and pigments-to comprehend but also the decoration on the pottery, analysis of design-elements, temporal and spatial relationships of types and "horizon styles" to pursue and to grasp. Small wonder that one can become involved and bogged down in minutiae and perhaps lose his way in a maze of technological and esthetic problems. Thus, it is difficult to persuade oneself not to make the study of pottery an end in itself. One can avoid this danger by integrating the ceramic data with all other available data and making interpretations from this combined information.

In this brief section I shall present the data that might conceivably be useful to other students who may wish to use this information for making other and different interpretations.

To supplement my remarks, I persuaded Miss Cronin and Mr. Freeman, graduate students in the Department of Anthropology, the University of Chicago, to pursue two investigations and to report on their efforts. Miss Cronin's investigation dealt with possible derivation of Snowflake Black-on-White pottery through the analysis of elements of designs. Mr. Freeman's study was concerned with statistical analysis of the painted pottery types recovered from the excavations and an ordering of the sites based on this analysis.

The reports of Miss Cronin and Mr. Freeman follow this chapter.

I have not discussed the method of manufacture - securing and preparing clays, shaping, polishing or finishing inasmuch as this problem has been well ventilated several times. Also omitted are such items as origins (except for the chapter on the lineage of Snowflake Black-on- 
White pottery), paste, tempering, paints, shapes, and decoration. A discussion of these would seem superfluous since all the types mentioned in the alphabetically arranged lists at the end of this chapter have been described and illustrated (see citations in Martin, Rinaldo, and Longacre, 1961, pp. 143-144). The exceptions are Gila Polychrome, for which I cite Haury (1945, pp. 63-80), and Snowflake Black-on-White, a type that we are not yet ready to describe.

The uses to which pottery was put may briefly be listed: in the preparation, cooking and serving of foods (the practice of providing separate containers or plates for each individual was unknown to these people); in the storage of water, foods, and seeds; and in ceremonial and mortuary rituals.

In other reports, we have frequently mentioned "trade" or "intrusive" pottery; in this chapter there is no such subdivision. If I possessed data derived from the technological methods of analysis (petrographic and other laboratory results obtained from thin sections) and if I could combine these with information regarding style and general appearances of pottery derived from direct inspection. I should be happy to make lists of possible intrusives. These data I do not have. Rare, unique or unfamiliar types may be possible intrusives, but I prefer to list them as unknowns until such time as I can avail myself of analytical methods of identifying ceramic materials. Such identification, however, may be slow and costly and it requires special laboratory equipment and a trained ceramist. These, unfortumately, are not always at hand.

In Freeman's report (chapter $\mathrm{IV}^{\prime}$ ) the author leaned heavily on pottery for relative dating of sites. The pottery types chosen for this purpose had already been dated elsewhere, for the most part by dendrochronology, and have a relatively short time span. This inferential method was chosen because (1) pottery possesses a variety of features and richness of development; and (2) it was the only one open to us for creating a chronological frame of reference, since we lacked absolute dates for any of the sites. The ordering of the sites is probably correct; assigning estimated dates to them is a complex matter, depending as it does on intangible factors, the prejudices of the authors, and unrecorded impressions gained from the excavations. The assignment of dates will be deferred to the last chapter.

Miss Cronin found herself involved in the question of ceramic change. Did the changes in design elements come about gradually or suddenly? Long experience in sorting and classifying sherds leads me to believe that pottery represents a continuous stream of development and that changes in design elements and style occurred gradually, for the most part, and 
that the potters were not aware that they were taking place. The mechanisms for producing changes in pottery designs are not well understood. It is often assumed that there is manipulation of designs resulting in freshness and variety of treatment (Bunzel, 1929, p. 57). Rands (1961, p. 333) suggests that in some traditions stylistic changes are made up of small innovations that are cumulative; or that minor changes are in the nature of substitutions rather than accumulations.

Whatever may have been the mechanisms for changes in pottery designs, one can sense a general drift throughout a larger area. Indeed, even though I can not document it, I advance the speculation that the trend in ceramic designs throughout much of the Southwest may have been "drifting" along the same general path at roughly the same time levels. In other words, we may have horizon styles (Willey, 1948, p. 8) in the Southwest.

A total of 15,243 sherds of all types was recovered from six sites. In chronological order, early to late, the frequencies are as follows:

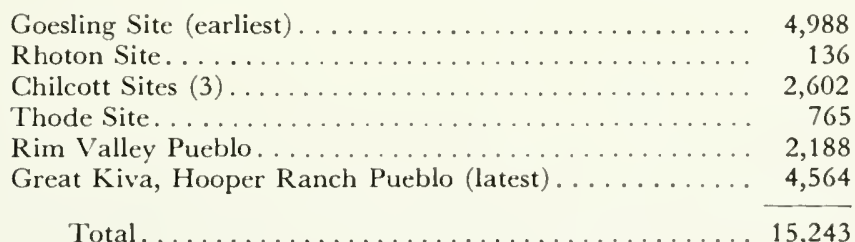

Tables showing total sherd tabulations and percentages for all sites (except Rhoton Site) are presented at the end of this chapter. We have not included any remarks on the pottery types or the architectural details of the Rhoton Site (a very small one) because one day's digging produced only a few sherds and not much else. Cronin and Freeman wanted, however, to include the Rhoton sherds in their analyses because the designs and types seemed pertinent.

Complete sherd counts for all rooms and levels have been published (Martin, Rinaldo, Longacre, and Freeman, 1961).

Someone may observe that the total number of sherds for the Hooper Ranch Pueblo (Great Kiva) as given here $(4,564)$ docs not agree with the number $(4,998)$ of sherds for the Hooper Ranch Pueblo as given by Freeman in his chapter. Mr. Freeman used the sherd count from the Great Kiva and also from some of the rooms of the Pueblo (dug in previous season, 1959; Martin, Rinaldo and Longacre, 1961). 


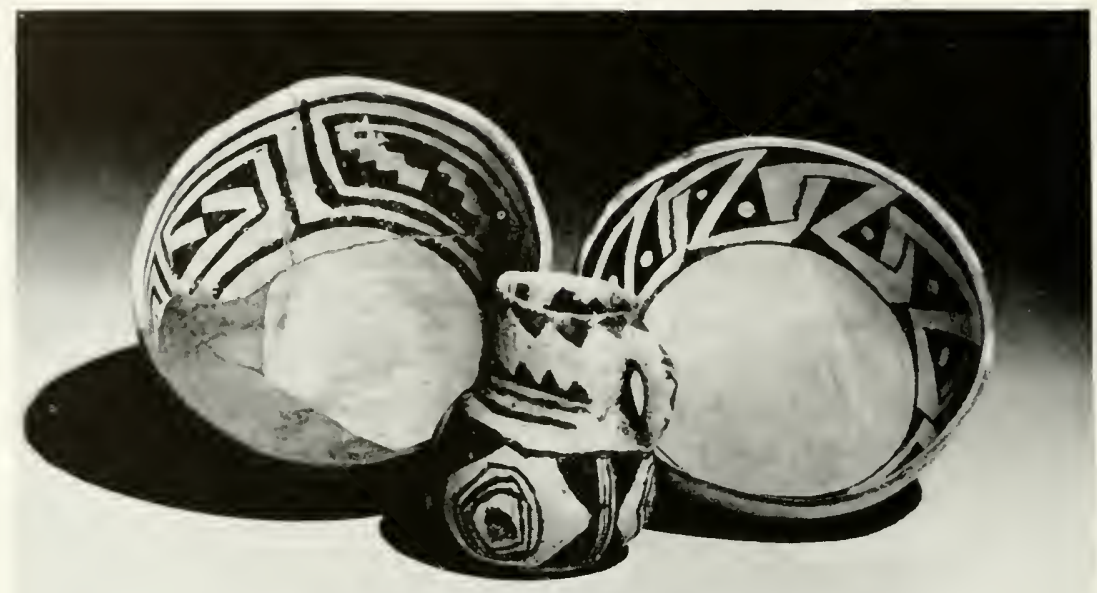

Fig. 43. Snowflake Black-on-White pottery.

\section{WHOLE OR RESTORABLE POTS RECOVERED ${ }^{1}$}

1. Red Mesa Black-on-White bowl (incipient Snowflake Black-onWhite?); cat. no. 280955; found in fill 2 of Pithouse A. Goesling Site (fig. 43, right).

2. Snowflake Black-on-White bowl (cat. no. 280954); found on floor of Room 1, Chilcott Site 1 (fig. 43, left).

3. Snowflake Black-on-White pitcher (cat. no. 280935); found near House 2, Chilcott Site 1.

4. Snowflake Black-on-White(?) pitcher (cat. no. 280936); found with burial no. 1, Room A, Thode Site (fig. 43, center).

5. Brown indented corrugated, smudged interior, bowl (cat. no. 280937); found with burial no. 1, Room A, Thode Site (fig. 44, right).

6. Brown indented corrugated, smudged interior, bowl (cat. no. 280938 ); found with burial no. 1, Room A, Thode Site.

7. Brown indented corrugated jar (cat. no. 280939): found with burial no. 1, Room A, Thode Site (fig. 44, left).

8. McDonald Corrugated bowl (cat. no. 280940); found on floor of Room B, Rim Valley Pueblo (fig. 45).

9. Woodruff Smudged bowl (cat. no. 280941); found on floor of Room G, Rim Valley Pueblo.

1 Iisted in approximate chronological order of sites; earliest sites given first and latest, last. 


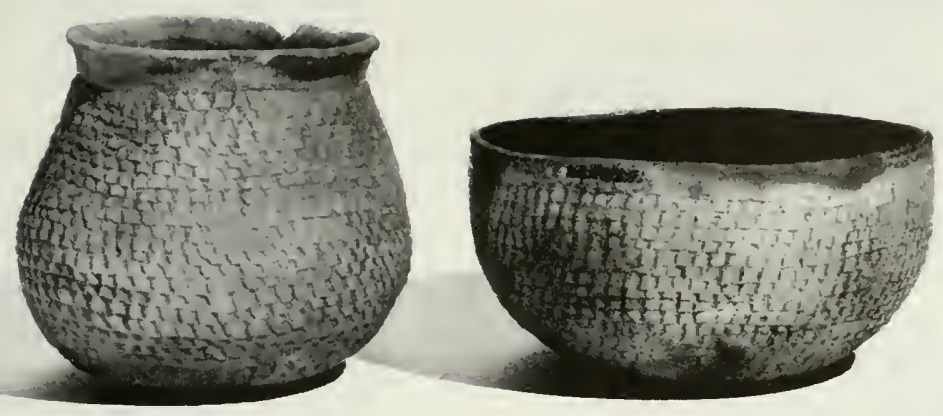

FiG. 44. Brown indented corrugated pottery.

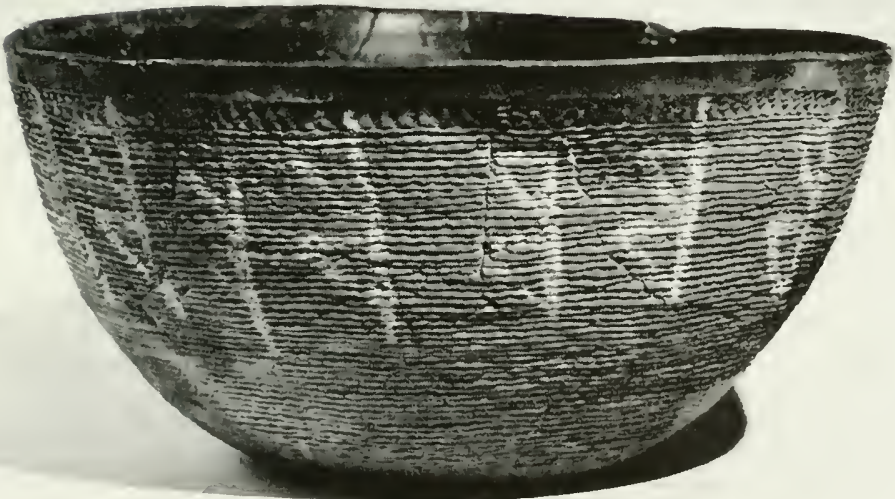

FIg. 45. McDonald Corrugated bowl. 
10. Heshota-uthla Polychrome(?) jar, miniature (cat. no. 280953); found in floor crypt with sacred stone image; Great Kiva, Hooper Ranch Pueblo.

\section{RELATIVE POPULARITY OF SEVERAL OF THE SIGNIFICANT PAINTED POTTERY TYPES}

These sites are listed according to the seriation or ordering as worked out by Freeman (Chapter IV).

1. Goesling Site; two pithouses dug:

$\%$

Red Mesa Black-on-White. . . . . . . . . . . . . . 27.35

Kiatuthlanna Black-on-White............... 4.63

2. Chilcott Sites; pithouses, early type surface rooms, and brush shelters:

$\begin{array}{lr} & \\ \text { Snowflake Black-on-White } \ldots \ldots \ldots \ldots \ldots \ldots \ldots \ldots \ldots \ldots \ldots & 19.39 \\ \text { Reserve Black-on-White } \ldots \ldots \ldots \ldots \ldots \ldots \ldots \ldots \ldots \ldots \ldots & 4.11 \\ \text { Tularosa Black-on-White } \ldots \ldots \ldots \ldots \ldots \ldots \ldots \ldots \ldots \ldots \ldots & 2.23 \\ \text { Kiatuthlanna Black-on-White } \ldots \ldots \ldots \ldots \ldots \ldots \ldots \ldots \ldots \ldots & 1.61\end{array}$

Out of total of 2602 sherds, 6 were Wingate Black-on-Red.

3. Thode Site; "incipient pueblo"; surface rooms with masonry walls and sub-surface floors, each room close to another but not contiguous:

$\begin{array}{ll}\text { Snowflake Black-on-White } \ldots \ldots \ldots \ldots \ldots \ldots \ldots \ldots \ldots \ldots \ldots & \begin{array}{r} \\ 23.00 \\ \text { Tularosa Black-on-White } \ldots \ldots \ldots \ldots \ldots \ldots \ldots \ldots \ldots \ldots\end{array} \\ \text { Reserve Black-on-White } \ldots \ldots \ldots \ldots \ldots \ldots \ldots \ldots \ldots \ldots & 2.61\end{array}$

Out of total of 765 sherds, 3 were Wingate Black-on-Red.

4. Rim Valley Pueblo; small pueblo consisting of two units, each of one story, totaling about 25 rooms. No kiva was located. Built near rim of canyon of Little Colorado River:

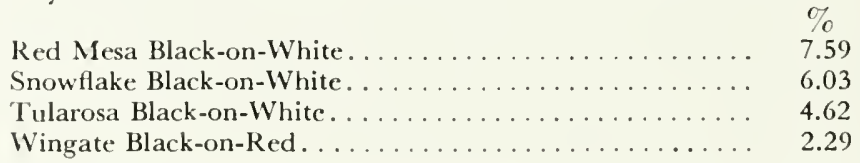

Out of total of 2,188 sherds, 3 were Houck Polychrome and 2 were St. Johns Polychrome.

5. Great Kiva, Hooper Ranch Pueblo (for description of architectural and ceramic details of pueblo, see Martin, Rinaldo and Longacre, 1961). 
Tularosa Black-on-White. . . . . . . . . . . . . . 11.00

Wingate Black-on-Red . . . . . . . . . . . . . . 6.77

St. Johns Polychrome... . . . . . . . . . . . . . . . . 3.13

Heshota-uthla Polychrome. . . . . . . . . . . . . . . 2.08

Four Mile Polychrome. . . . . . . . . . . . . . . . . 2.02

Kwakina Polychrome...................... 1.88

TABLe 1.-TOTALS OF SHERDS, GOESLING SITE

Decorated Wares

No. ${ }_{0}$

Kiatuthlanna Black-on-White. . . . . . . . . . . . $231 \quad 4.63$

Red Mesa Black-on-White. . . . . . . . . . . . . . . . . 136427.35

White Mound Black-on-White. . . . . . . . . . . . . . . $10 \quad .20$

Wingate Black-on-Red . . . . . . . . . . . . . . . . . 12

Indeterminate Black-on-White. . . . . . . . . . . $579 \quad 11.61$

Total for Decorated Wares. . . . . . . . . . . . . . . $\overline{2185} \overline{43.81}$

\section{Textured Wares}

Brown plain corrugated ........................ $2 \quad 2.04$

Brown indented corrugated ..................... 3.06

Gray plain corrugated. . . . . . . . . . . . . . . . . . $185 \quad 3.71$

Gray indented corrugated . . . . . . . . . . . . . . . . $110 \quad 2.21$

Gray corrugated, wavy or exuberant .............. $61 \quad 1.22$

Incised corrugated . . . . . . . . . . . . . . . . . $28 \quad .56$

Kana-a Gray. . . . . . . . . . . . . . . . . . . . . $460 \quad 9.22$

Punched corrugated. . . . . . . . . . . . . . . . . . . . $11 \quad 22$

Reserve corrugated. . . . . . . . . . . . . . . . . . . . . . . 29.04

Gray corrugated bottoms . . . . . . . . . . . . . . . $1555 \quad 31.18$

Total for Textured Wares................... $2417 \quad 48.46$

\section{Plain Wares}

Alma Plain. . . . . . . . . . . . . . . . . . . . . . . . . . . . $120 \quad 2.41$

Lino Gray. . . . . . . . . . . . . . . . . . . . . . . . . . $43 \quad 436$

Reserve Smudged.......................... $145 \quad 2.91$

San Francisco Red, Smudged Interior . . . . . . . . . . . . . 192

Pink Lino Grey. . . . . . . . . . . . . . . . . . . . . . . $77 \quad 1.54$

Total for Plain Wares................... $386 \quad \overline{386} \quad \overline{7.74}$

Total for Goesling Site. . . . . . . . . . . . . . . . . . . . . 4988 100.01 
Decorated Wares

No. To

Kiatuthlanna Black-on-White.................. $42 \quad 1.61$

Red Nesa Black-on-White. . . . . . . . . . . . . . . $15 \quad .58$

Reserve Black-on-White. . . . . . . . . . . . . . . . . $107 \quad 4.11$

Snowflake Black-on-White. . . . . . . . . . . . . . . . $505 \quad 19.39$

Tularosa Black-on-White. . . . . . . . . . . . . . . . $58 \quad 2.23$

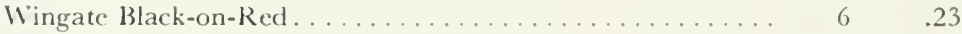

Indeterminate black-on-red. . . . . . . . . . . . . . . . $11 \quad 42$

Indeterminate black-on-white. . . . . . . . . . . . . $524 \quad 20.12$

Total for Decorated Wares.................... $1268 \quad 48.69$

Textured Wares

Brown plain corrugated. . . . . . . . . . . . . . . . . . $14 \quad .54$

Brown plain corrugated, smudged interior .......... 5.19

Brown indented corrugated . . . . . . . . . . . . . . . $1016 \quad 39.01$

Brown indented corrugated, smudged interior......... $51 \quad 1.96$

Gray plain cortugated..................... $1 \quad .04$

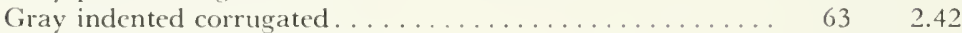

Gray patterned corrugated ................... 6 .23

MíDonald Corrugated Indented ................ 7 .27

Patterned corrugated ......................... $69 \quad 2.65$

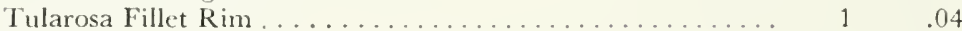

Indeterminate......................... $35 \quad 1.34$

Indented corrugated, red slip interior and exterior . . . . . $6 \quad 6 \quad 23$

Indented corrugated exterior, black-on-white interior . . . . $2 \quad 2 \quad .08$

Brown indented corrugated, fugitive red paint interior..... $1 \quad .04$

Total for Textured Wares.................... $1277 \quad 49.04$

Plain Wares

Alma Plain. ............................... $32 \quad 1.23$

Forestdale Smudged. . . . . . . . . . . . . . . . . . . . . . 1 . 14

Rescrve Smudged........................... $2 \quad .08$

San Francisco Red....................... $2 \quad .08$

Woodruff Smudged ......................... 7 . 72

Indeterminate. ......................... 28

Plain grey, not decorated or slipped............... $11 \quad .42$

Total for Plain Wares ..................... $57 \quad 2.20$

Total for Chilcott Sites . . . . . . . . . . . . . . . . . . . . 260299.93 
TABLE 3.-TOTALS OF SHERDS, THODE SITE

Decorated Wares

No. $\quad T_{0}$

Kiatuthlanna Black-on-White. . . . . . . . . . . . $16 \quad 2.10$

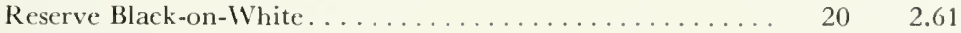

Snowflake Black-on-White. . . . . . . . . . . . . . . $176 \quad 23.00$

Tularosa Black-on-White..................... $32 \quad 4.18$

Wingate Black-on-Red. . . . . . . . . . . . . . . . . . . . . 39

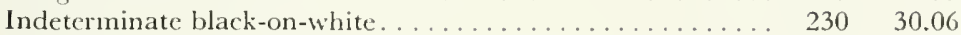

Total for Decorated Wares................. $\overline{477} \overline{62.34}$

Textured Wares

Brown plain corrugated. . . . . . . . . . . . . . . . . . . $15 \quad 1.96$

Brown plain corrugated, smudged interior........... 13

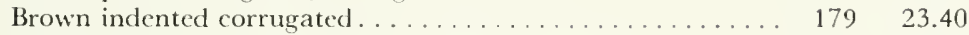

Brown indented corrugated, smudged interior.......... 2 .26

Gray indented corrugated . . . . . . . . . . . . . . . . 26

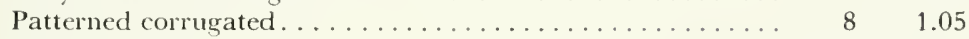

Indeterminate. . . . . . . . . . . . . . . . . . . . 39

Total for Textured Wares................ $210 \quad 27.45$

Plain Wares

Alma Plain................................ $51 \quad 6.67$

San Francisco Red............................. 39.39

Woodruff Smudged . . . . . . . . . . . . . . . . . . . . . . . . $4 \quad .52$

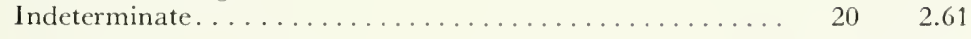

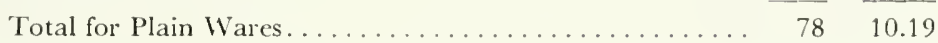

Total for Thode Site......................... $765 \quad 99.98$ 
'TABle 4.-TO'TALS OF SHERDS, RIM VALLEy PUEBLO

Decorated Wares

No. $\%$

Houck Polychrome. . . . . . . . . . . . . . . . . . . . . . $33^{14}$

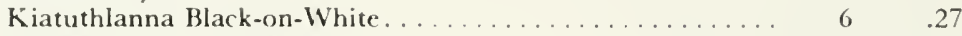

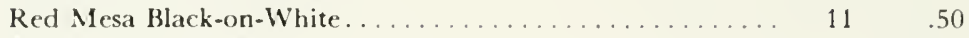

Reserve Black-on-White. . . . . . . . . . . . . . . . . . . $166 \quad 7.59$

St. Johns Polychrome. . . . . . . . . . . . . . . . . . . . 22.09

Snowflake Black-on-White. . . . . . . . . . . . . . . $132 \quad 6.03$

Tularosa Black-on-White. . . . . . . . . . . . . . . . . . . . $101 \quad 4.62$

Tularosa White-on-Red. . . . . . . . . . . . . . . . . . . $2 \quad .09$

Wingate Black-on-Red. . . . . . . . . . . . . . . . . . $50 \quad 2.29$

Indeterminate black-on-red . . . . . . . . . . . . . . . 87

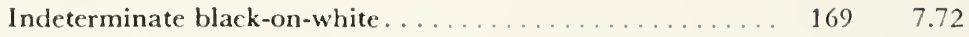

Total for Decorated Wares................... $650 \quad 29.71$

Textured Wares

Brown plain corrugated.................... 76

Brown plain corrugated, smudged interior . . . . ...... 107

Brown indented corrugated . . . . . . . . . . . . . . 528

Brown indented corrugated, smudged interior. . . . . . . . 506

Gray indented corrugated . . . . . . . . . . . . . . 1

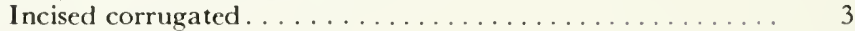

McDonald Corrugated Plain . ................. 6

McDonald Corrugated Indented . . . . . . . . . . . . . . . . 19

McDonald Corrugated Patterned.................. 10

Patterned corrugated . . . . . . . . . . . . . . . . . . 58

Punched corrugated ...................... 4

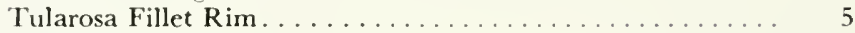

Zoned corrugated ......................... 3

Indeterminate . . . . . . . . 35

Total for Textured Wares.................... 1361

Plain Wares

Alma Plain............................. 33

Reserve Smudged. . . . . . . . . . . . . . . . . . . . . .

San Francisco Red. . . . . . . . . . . . . . . . . . . . .

San Francisco Red, Smudged Interior. . . . . . . . . . . . .

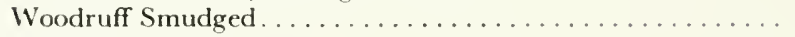

Indeterminate . . . . . . . . . . . . . . . 4

Total for Plain Wares.................... 177

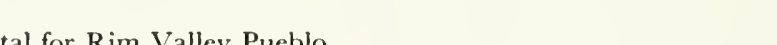




\section{TABLE 5.-TOTALS OF SHERDS, GREAT KIVA, HOOPER RANCH PUEBLO}

Dccorated Wares

Four Mile Polychrome. . . . . . . . . . . . . . . . . . . 92

Gila Polychrome........................ 11

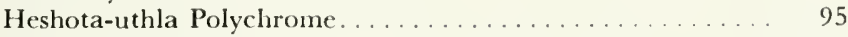

No. Yo

Houck Polychrome. . . . . . . . . . . . . . . . . . . 10

Kwakina Polychrome........................ 86

Kiatuthlanna Black-on-White. . . . . . . . . . . . .

Lino Black-on-Gray. . . . . . . . . . . . . . . . . . . . . . 3

Pinedale Black-on-Red . . . . . . . . . . . . . . . . . . . . 19

Pinedale Polychrome. . . . . . . . . . . . . . . . . . 73

Pinnawa Glaze-on-White . . . . . . . . . . . . . . . . . 7

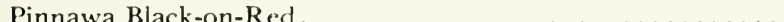

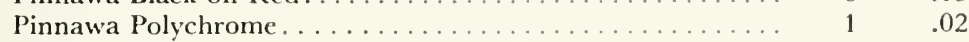

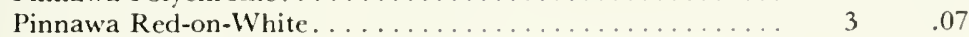

Querino Polychrome......................... 5 . 5

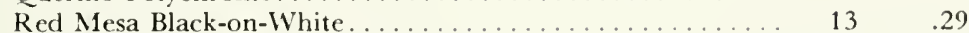

Reserve Black-on-White. . . . . . . . . . . . . . . . . . . . $12 \quad .26$

St. Johns Polychrome. . . . . . . . . . . . . . . . . . . . $143 \quad 3.13$

Show Low Black-on-Red. . . . . . . . . . . . . . . . . . . $3 \quad .07$

Snowflake Black-on-White. . . . . . . . . . . . . . . . $15 \quad .33$

Springerville Polychrome. . . . . . . . . . . . . . . . $11 \quad .24$

Tularosa Black-on-White. . . . . . . . . . . . . . . . $502 \quad 11.00$

Wingate Black-on-Red . . . . . . . . . . . . . . . . . . . $309 \quad 6.77$

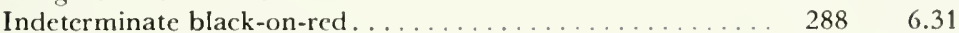

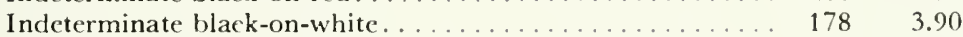

Indeterminate white-on-red . . . . . . . . . . . . . . . . . $6{ }^{6}$

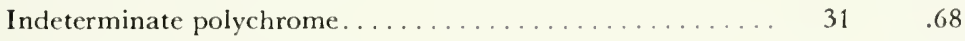

Total for Decorated Wares... . . . . . . . . . . . . . . . $1925 \quad 42.19$

Textured Wares

Alma Scored.............................. $2 \quad .04$

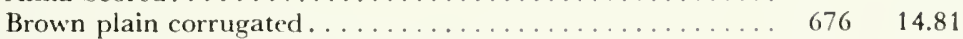

Brown plain corrugated, smudged interior. . . . . . . . . . $172 \quad 3.77$

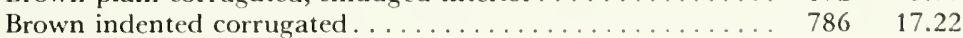

Brown indented corrugated, smudged interior.......... $408 \quad 8.94$

Incised corrugated . . . . . . . . . . . . . . . . . . . . . $22 \quad .48$

McDonald Corrugated Indented . . . . . . . . . . . . . . . $13 \quad .29$

McDonald Corrugated Patterned. . . . . . . . . . . . . . . 192

Patterned corrugated . . . . . . . . . . . . . . . . . . . . $91 \quad 1.99$

Punched corrugated ........................ $12 \quad .26$

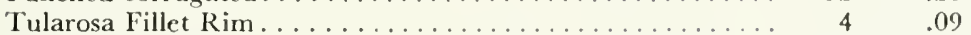

Zoned corrugated .......................... $4 \quad .49$

Indeterminate..................... $153 \quad 3.35$

Total for Textured Wares. . . . . . . . . . . . . . . . . . . $2344 \quad 51.35$ 


\section{TABLE 5. TOTALS OF SHERDS, GREAT KIVA, HOOPER RANCH PUEBLO (continued)}

\begin{tabular}{|c|c|c|}
\hline Plain Wares & No. & $\%$ \\
\hline Alma Plain & 142 & 3.11 \\
\hline Forestdale Smudged $\ldots \ldots \ldots \ldots \ldots \ldots \ldots \ldots \ldots$ & 12 & .26 \\
\hline Reserve Smudged. . . . . . . . . . . . . . . . . . . & 19 & .42 \\
\hline San Francisco Red. . . . . . & 42 & .92 \\
\hline San Francisco Red, Smudged Interior............... & 7 & .15 \\
\hline Woodruff Smudged. . . . . . . . . . . . . . . . . . . . & 53 & 1.16 \\
\hline Indeterminate $\ldots \ldots \ldots \ldots \ldots \ldots \ldots \ldots \ldots \ldots \ldots$ & 19 & .42 \\
\hline Burnished interior, brown ware; not smudged . . . . . . . . & 1 & .02 \\
\hline Total for Plain Wares. & 295 & 6.46 \\
\hline
\end{tabular}




\title{
IV. Statistical Analysis of Painted Pottery Types from Upper Little Colorado Drainage
}

\author{
By Leslie G. Freeman, Jr. \\ Research Assistant, Department of Anthropology \\ University of Chicago
}

\section{INTRODUCTION}

This study was designed to discover whether interpretations more far-reaching than those yielded by our former methods of analysis could be derived from the data at hand from our six sites, without incurring prohibitive expenditures of time and money. The ideal tool for the study had to be one which did not require the intervention of many operations between the raw data as represented by our archaeological collections and the conclusions which could be expected to result. For this reason, a statistical study immediately suggested itself. The particular method chosen as best suited to the task and the data was the Robinson-Brainerd seriation technique (Robinson, 1951: Brainerd, 1951).

Some criticism of the Robinson-Brainerd method was offered by Lehmer (1951), when the technique was proposed. The RobinsonBrainerd technique does not correct for differences in sample size, and Lehmer proposed that this could be remedied by operating with mean standard errors, instead of the original "coefficients of similarity." Both the original method as presented by Robinson (1951) and the revision proposed by Lehmer have inherent advantages and disadvantages, which I shall not attempt to evaluate. I have chosen to use the method as originally presented, since Lehmer's method requires random sampling, or at least a definition of the universe from which the samples are drawn. Our data do not meet these requirements, so the use of parametric statistics in their analysis cannot be justified. In addition, the calculations involved in the original Robinson-Brainerd technique are simpler than those proposed by Lehmer, and the rationale behind the operations is easier for the non-statistician to follow. 


\section{CHOICE OF MATERIALS}

It was determined that the materials which best fitted the RobinsonBrainerd method were the painted wares. The types of painted pottery represented in our collections are fairly numerous, and the types themselves are well enough fixed for the purposes of a seriation. Further, individual types have a temporal existence so limited that one can expect some change in popularity of a given type over a fairly short time period. Some types are well established as horizon markers, and one can date their appearance and disappearance from an assemblage quite well, so that they provide an internal check on the results of any supposedly chronological ordering imposed upon them.

Limiting the analysis to painted wares had some drawbacks for I was forced to use small samples in the seriations, but I felt that the advantages inherent in the consideration of painted wares alone outweighed the disadvantages of so doing. Had time permitted, it would have been desirable to reseriate the materials, including some at least of the available utility wares. Since this was not done, I have no idea whether or not it would have yielded the same results, or better or worse ones. It would certainly be worth while to undertake such a study in the future.

\section{SOME METHODOLOGICAL CONSIDERATIONS}

The temporal sequence of the sites themselves could be closely approximated by inspection, but though the ends of the sequence were easily recognizable, the relative temporal order of the Rhoton, Thode, and Chilcott sites was not as evident. I hoped that the seriation would establish a finer chronological sequence than could be drawn by eye, and that once this sequence had been established our attention would be drawn to other factors causing differences between samples. Ideally, if the seriation of a number of samples is correct, any abrupt discontinuity in the materials of one sample compared to the rest, if it is inexplicable by considerations of stylistic change in a single tradition over time, can be due to other factors. Some of these factors are known. The intrusion of a tradition foreign to the area concerned is one. The looting of abandoned sites by culturally dissimilar groups, for example, to procure potsherds for tempering materials, is another. The preservation of obsolete materials as heirlooms, and the mere collection of curiosities have also been suggested (Martin and Rinaldo, 1960b, pp. 206-208). While realizing that all the possible factors are not yet known, I hoped that once temporal considerations are controlled, the nature of some such discontinuities would point out one or more possibilities as the more likely causal factor. 
My main interest, then, lay first, in establishing the relative chronological order of the sites; second, in establishing the relative chronological order of the rooms within each site. Therefore, I seriated not only the sites, but the floor materials from each site. Each seriation yielded a probable temporal order of materials, but to determine which end of the sequence was late and which early, the seriation had to include some samples whose relative chronological position was known. Where it was possible, collections from superimposed floors were used, but often I had to include material from a floor and the fill above it to get direction from the seriation. I tried to avoid using fill samples, since they might have accumulated over long periods of time and for that reason might have proven difficult to fit into the seriation. Collections from fills were purposely used in cases where the number of floor samples was so small that the trends in pottery popularity through time based on floor materials alone would have appeared meaningless.

My lower working limit of size of sample in this study was 23 sherds. Besides the fact that sample size was limited when I restricted consideration to painted wares, large samples often had to be ignored because they behaved as mixed samples in the seriation. In some cases, this may have been due to the continued occupation of a floor throughout the time period represented by the rest of the samples, so that materials from every period were represented in the floor in anomalous proportions. Also, my desire to consider as much floor material as possible often caused me to accept small samples, as the excavated material from floors included fewer sherds than that from fills. Since the samples were so small, I feel that the results of the seriations alone should not be used as anything other than possible corroborating evidence for interpretations drawn from other data, and clues to further investigation. The seriations in themselves do not warrant even the statement of probability of correctness usually made in statistical studies.

The conclusions drawn in this study refer primarily to the seriated materials. The study would be of little value, however, if the results of the seriation had no reference except to the seriated materials. We must assume that the painted ware samples from floors, at least, are part of pottery assemblages which are correlated with assemblages of other cultural materials. We assume, then, that inferences drawn from the painted ware samples hold generally true for the occupations they represent. The same assumption cannot be made about fill materials. They may, of course, represent more than one occupation. However, the chronological position of these occupations will be that of the mixed painted ware collection representing them. Still, one cannot extend 
the inferences drawn from the seriated materials to statements about the occupation of the sites as a whole; they are directly applicable only to the structures and areas excavated.

Before proceeding to the actual seriation, I made use of a method which was first developed by Arthur J. Jelinek (1960) for indicating graphically the relative positions of the samples. This method consists in the construction of a "map" on which the relative similarity among the samples is indicated by their position and the nature of the lines joining them (fig. 46). The location of the samples and the nature of their connections are determined by the respective sizes of the RobinsonBrainerd coeflicient of similarity between any two samples. The maps allow one to see at a glance the inter-relationships among samples, and are amenable to both temporal and non-temporal interpretation. Without this method my task would have been materially more difficult. I relied on it 10 yield a first approximation to the seriations. (See Martin, Rinaldo, Longacre and Freeman, 1961, for analysis of sherds on which seriations are based.)

\section{BASIC PROCEDURE}

The percentage of each type of painted pottery in each sample was calculated. Only sherds which could be definitely classified were used in this calculation. Each sample was then compared with every other sample. The differences in percentage of each type between the two samples were added, giving a total difference between the two samples. Now the maximum possible difference between two samples is 200 . Two samples would be this different if 100 per cent of the pottery in sample 1 were of different types than 100 per cent of the pottery in sample 2. The calculated total difference was subtracted from the maximum possible difference to give the coefficient of similarity.

The coefficients of similarity were then placed in a symmetrical matrix. In this matrix, the diagonals, left blank, are relationships of identity, so that the blanks represent coefficients of 200 (the maximum similarity). The ideal arrangement of samples in this matrix shows the highest coefficients on the diagonal, and decreasing coefficients to the upper right and lower left corners. This ideal was approached as closely as possible.

The temporal direction of the inter-site seriation was determined by the presence or absence of early and late painted ware types in the end samples. The direction of the intra-site seriations was determined by the positions of one or more floor samples relative to their respective fill samples, or by the relative positions of superimposed floors from the same room. Lastly, graphs of the popularity of each pottery type 


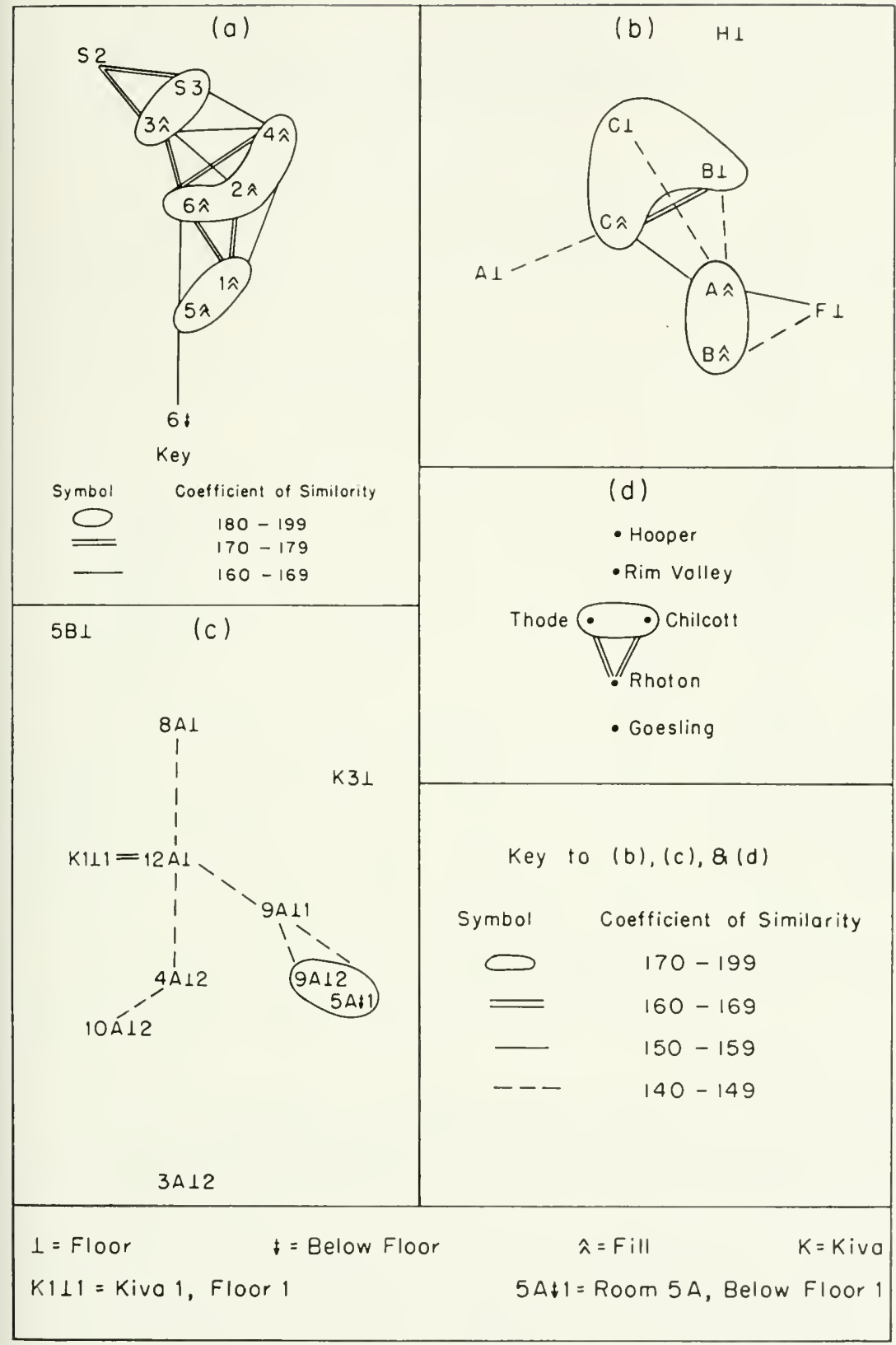

FiG. 46. Schematic illustration of the relative similarity between samples of pottery. (a) Chilcott sites; (b) Rim Valley Pueblo; (c) Hooper Ranch Pueblo; and (d) site totals. 


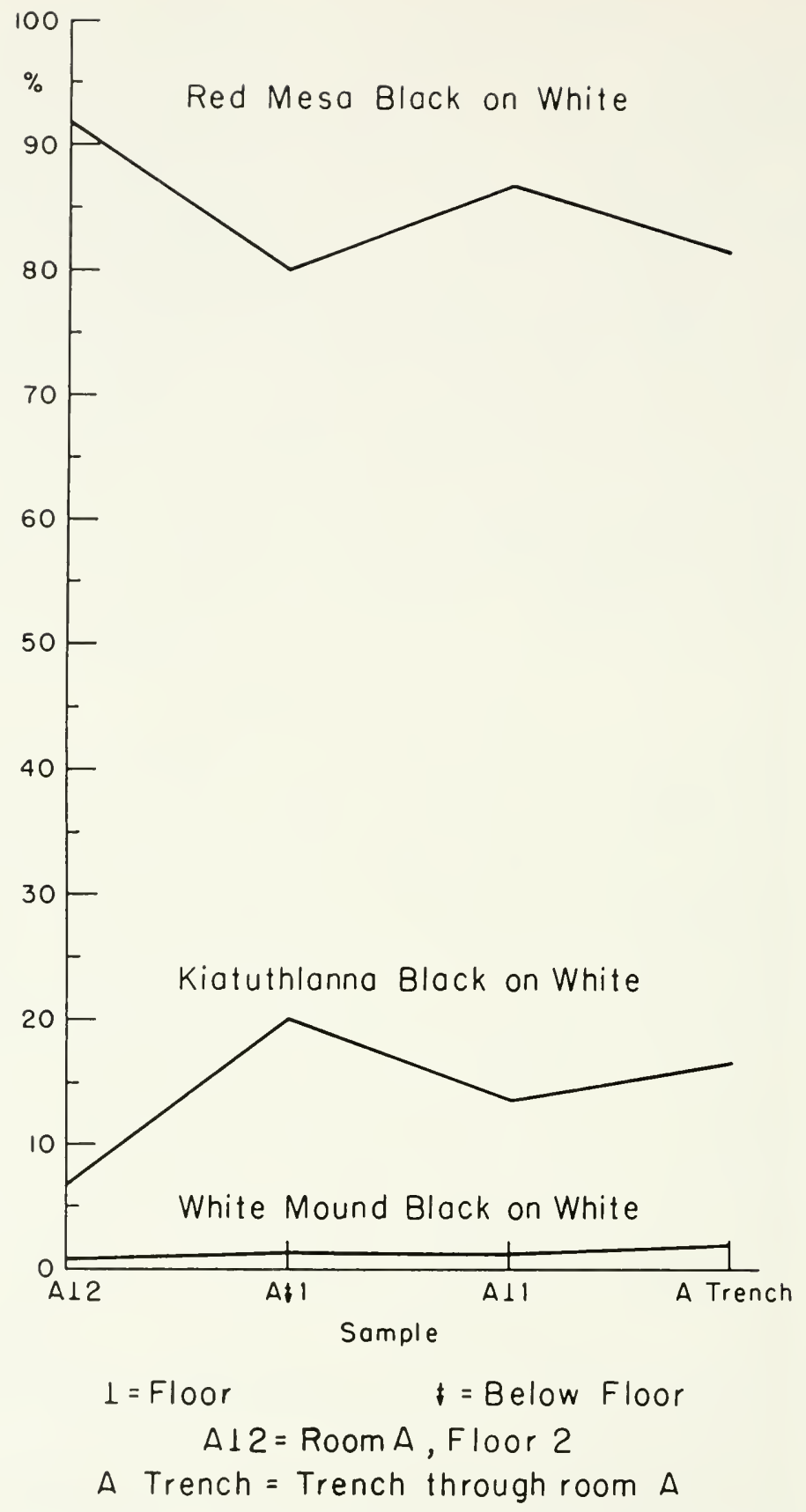

FIG. 47. Percentages of three pottery types by levels at the Goesling Site. 
based on the percentage of each type in the total painted ware assemblage in each sample were constructed, so that the fluctuations in popularity of each type through time could be observed.

\section{THE INTER-SITE SERIATION}

The map shows the relationships of the site samples (fig. 46, d). Sample sizes and the final seriation matrix for this ordering appear below.

\section{TABLE 6.-SAMPLE SIZE OF SHERDS AND FINAL MATRIX FOR IN'TER-SITE SERIATION}

\begin{tabular}{|c|c|c|}
\hline Site & Sample Size & $\begin{array}{c}\text { Number } \\
\text { of } \\
\text { sherds }\end{array}$ \\
\hline Goesling. & & 1587 \\
\hline Rhoton. & & 41 \\
\hline Chilcott. & & 695 \\
\hline Thode... & & 233 \\
\hline Rim Vall & & . 418 \\
\hline Hooper. . & & . 4998 \\
\hline
\end{tabular}

Final Matrix

$\begin{array}{lcccccc} & \text { Goesling } & \text { Rhoton } & \text { Chilcott } & \text { Thode } & \text { Rim Valley } & \text { Hooper } \\ \text { Goesling....... } & - & 24 & 15 & 12 & 5 & 3 \\ \text { Rhoton........ } & 24 & - & 167 & 165 & 84 & 17 \\ \text { Chilcott........ } & 15 & 167 & - & 183 & 108 & 20 \\ \text { Thode......... } & 12 & 165 & 183 & - & 105 & 49 \\ \text { Rim Valley.... } & 5 & 84 & 108 & 105 & - & 71 \\ \text { Hooper........ } & 3 & 17 & 20 & 49 & 71 & -\end{array}$

Due to the appearance of late pottery types at the Hooper Ranch, the order is from Goesling (early) to Hooper (late).

\section{INTERPRETATION}

The Goesling Ranch Site and the Hooper Ranch Site are at opposite ends of the seriational scale, and both show little similarity in painted ware collection to the rest of the sites or to each other. The Chilcott Ranch Site and the Rhoton and Thode Ranch Sites show much more similarity among themselves than any of them shows to any other site. We seem to have an early site, Goesling. separated widely in time and cultural affiliations from a group of three sites, Rhoton, Chilcott, and Thode. Separated from them by relatively great divergences in sherd collection is the Rim Valley Site, which, however, resembles them more than it does Hooper. This is the more striking since the geographic 
distance from the Hooper Ranch Site to the Rim Valley Site is very short, and one might have expected that the Rim Valley Site and Hooper Ranch Site could have been occupied coterminously by people with much the same cultural apparatus. How much such differences are due to non-random representation of the total settement at each site in the excavated material is impossible to determine, but the gap exists between the excavated portions of the two settlements.

\section{THE INTRA-SITE SERIATIONS}

The Goesling Site.-This site was not seriated internally, as only threc unmixed samples were available and all three were from Room $1 \mathrm{~A}$ : material from Floor 2, a collection of sherds from the fill between Floors 2 and 1, and the later material from Floor 1 itself. The percentages of each of the three major types of painted ware are shown in figure 47; one type, Wingate Black-on-Red, was excluded, since it is represented by only one sherd, which was found in a mixed collection from Room A, level 2, including both floor and fill material. Of the remaining three pottery types, Red Mesa Black-on-White forms early a large percentage of the total assemblage of painted wares and this percentage declines somewhat in time. Kiatuthlanna Black-on-White makes up only 7 per cent of the total painted ware assemblage on Floor 2, increasing to 16 per cent on Floor 1. White Mound Black-on-White constitutes an almost negligible percentage of the assemblage throughout time (less than 1 per cent).

\section{TABLE 7.-SAMPLE SIZE OF SHERDS FROM GOESLING SITE}

Room Number
of

A, Floor 2............................... 307

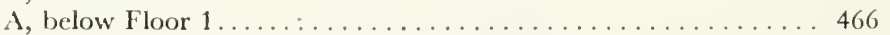

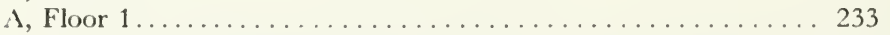

Trench through Room A........................ 454

(Floor 1 is the upper floor)

The Goesling Site is relatively homogeneous, but it must be remembered that only two floors in one room are represented in the Goesling samples, and the materials represent a single, short cultural horizon.

The Rhoton and Thode Sites.-Material from these two sites was not amcnable to seriation, except in the inter-site comparisons. It is largely surface and fill materials, which could not be ordered well, alone. The Thode Ranch Site materials are all from room fills, with no large samples 
from floors and thus do not possess the internal stratigraphic relationships necessary to determine the direction of the seriation.

The Chilcott Site. - This is actually three sites. Since Site 3 and Site 2 show a much greater degree of similarity in painted ware assemblage than either does to Site 1, it was determined to treat Sites 3 and 2 as units, mixed though they are, in the seriation of the materials from Site 1.

In Site 1, the only definitely unmixed sample is that of Room 6, below the floor. However, since it was felt that graphs of pottery popularity were desirable for the Chilcott Site, fills from both Room 6 and all other rooms were seriated. The results and sample size are shown below.

TABLE 8.-SAMPLE SIZE OF SHERDS FROM CHILCO'T'T SITE AND FINAL MATRIX

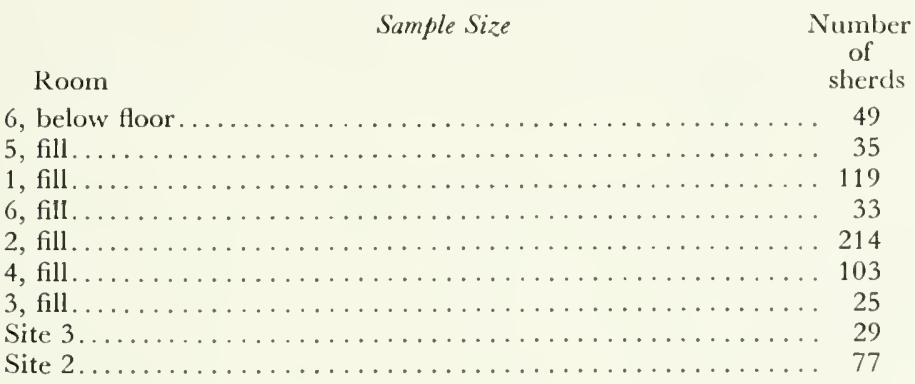

Final Matrix

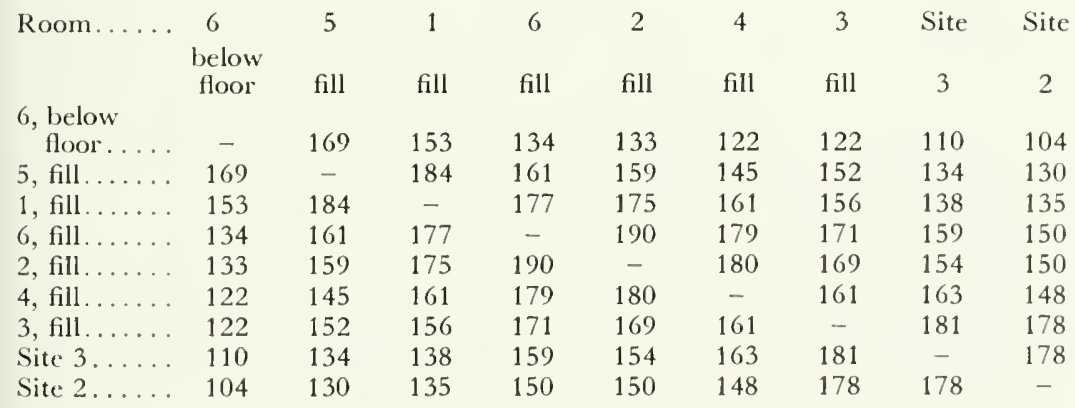

It will be noted that there is a high degree of similarity between each sample and the samples immediately adjacent to it. The excavated areas represented in the seriation seem to show a rather uniform direction of change in painted ware assemblage, which one would expect, if a single cultural group had occupied the three sites during the time perioct here represented. The uniformity of change, it must be remembered, 
may be non-temporal, and a function solely of the representation of the assemblages we have from the excavation. It is, however, hard to see why any other factor beyond temporal change need be called upon to explain the seriation results. Further, the fact that this uniformity is shown on the pottery graphs, with all types changing in quite regular fashion, adds weight to the significance of the results and makes it less likely that they are purely accidental.

The position of the materials from Room 6, below the floor, and the fill in Room 6 in the seriation make it reasonable to assume that the materials below the floor in Room 6 are early, and those in the fill of Room 3 are late in Site 1. The seriation also places materials from Site 3 and Site 2 as later than any from Site 1 and justifies the conclusion that Site 2 is later than Site 3. We also note that all the excavated rooms on Site 1 had filled or been filled before the materials from Sites 3 and 2 accumulated. Since the collections from Sites 3 and 2 are all fill or surface materials it is possible that late Site 1 and Site 3 were occupied contemporaneously.

Figure 48, $a$ and $b$, shows the percentage of each type of painted ware in the total painted ware assemblage in each sample. The pereentage of Wingate Black-on-Red has been omitted, since it occurs sporadically, late, and in very small quantities (only 4 sherds on the whole site). The graph shows the increase of Snowflake Black-on-White from 46 per cent to 90 per cent, the decrease over time of Reserve Blackon-White from 44 per cent to only 2 per cent, and the appearance of Kiatuthlanna Black-on-White, its climb in popularity to 11 per cent of the total late in Site 1, and its absence from Sites 3 and 2. Tularosa Black-on-White is present in small proportions of the total assemblage throughout the sites. The presence of Kiatuthlanna Black-on-White here is problematical. It is chronologically out of place. Arguments that it appears due to the discovery of an abandoned site, whence it was brought to the Chilcott Site to serve as tempering material, seem to me unconvincing. If sherd temper were being used, enough broken local material should have been available for use by the time Kiatuthlanna Black-on-White appears on the site. Furthermore, one would expect that the curve for Kiatuthlanna Black-on-White would not show the gradual increase that it does, but rather either a fairly uniform percentage or a random increase and decrease, if for some reason it were a popular tempering material and constantly available. I would be able to reconcile an isolated peak or two on the graph with the discovery of small quantities of Kiatuthlanna Black-on-White by one or two fortunate Chilcott residents, but it seems to me that an explanation of the present curve 


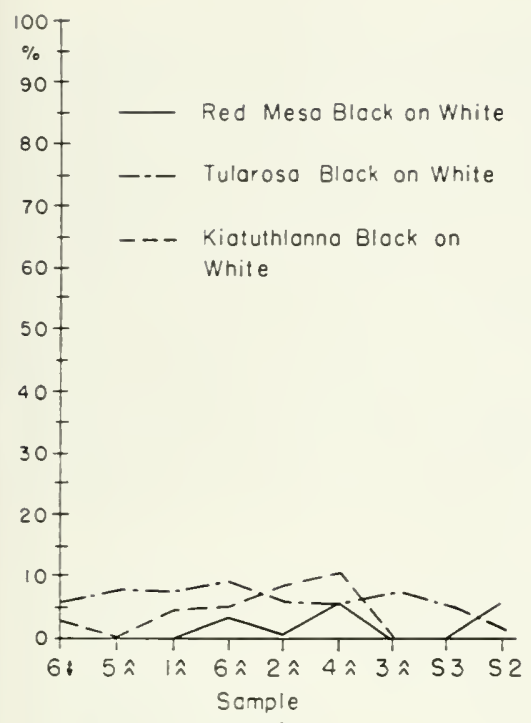

(a)

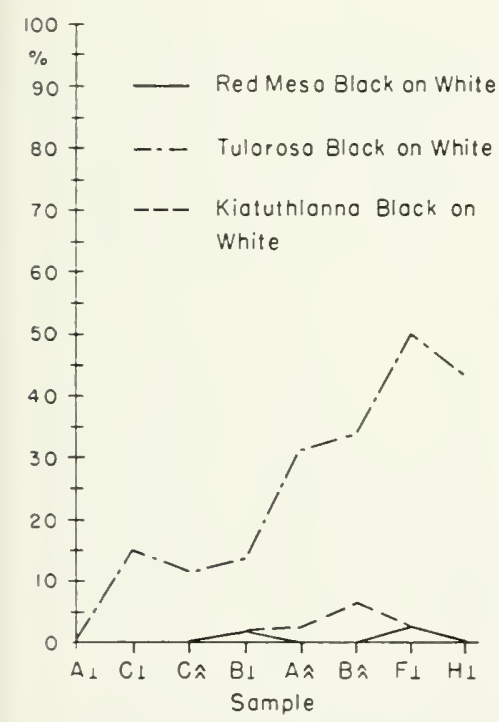

(c)

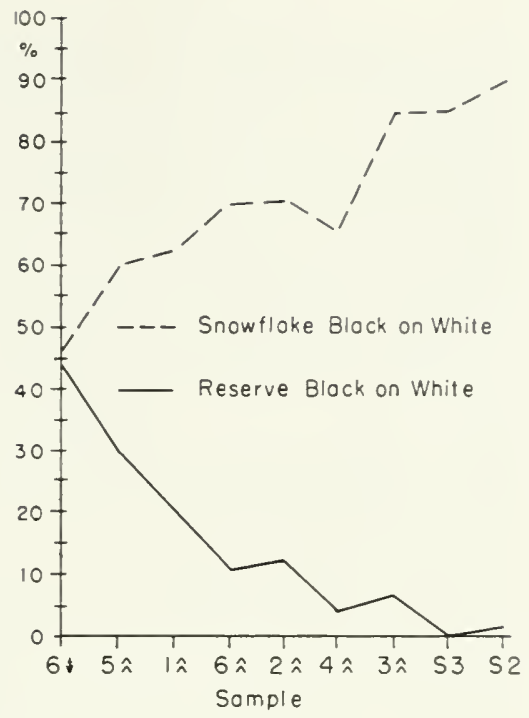

(b)

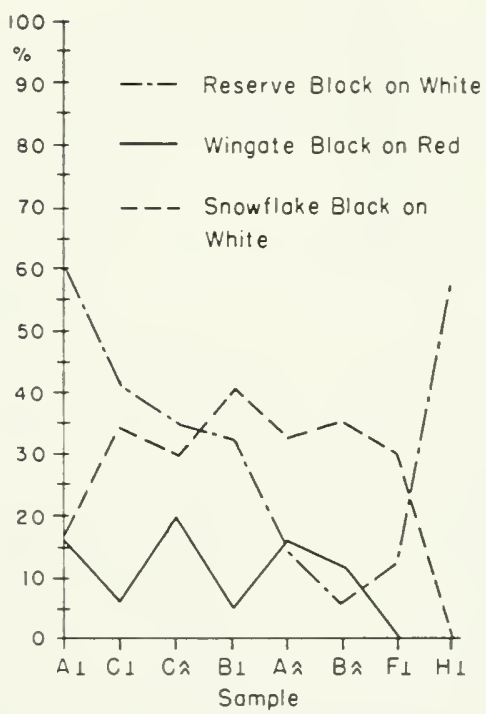

(d)

$\mathrm{S}=$ Site

$\perp=$ Floor

$t=$ Below Floor

$\hat{n}=$ Fill Moterial
$6 t=$ Room 6 , Below Floor
$B \hat{n}=$ Room B, Fill
S3 $=$ Site 3

Fig. 48. Trends in painted pottery types illustrated by samples from Chilcott Sites $(a$ and $b$ ) and Rim Valley Pucblo $(c$ and $d)$. 
on that basis requires too many hypothetical conditions. There remain a number of possibilities, among which one must mention the heirloom hypothesis, and the possibility of the long-continued manufacture of this pottery type by an individual or group, perhaps a clan. These suggestions are highly speculative, and if this were the only site where Kiatuthlanna appeared in an anomalous setting. I should be inclined to cliscard them all, or aecept the first over the other two.

The Rim Valley Pueblo.-Neither the Rim Valley Pueblo nor the Hooper Ranch Pueblo materials yielded a particularly satisfactory seriation. Figure 46, $b$, shows the mapped interrelationships among the Rim Valley samples. The seriation was based on 8 samples. The sample size and final seriation matrix are shown below.

\section{TABLE 9. SAMPLE SIZE OF SHERDS FROM RIM VALLEY PUEBLO AND FINAL MATRIX}

\begin{tabular}{|c|c|c|}
\hline Room & Sample Size & $\begin{array}{l}\text { Number } \\
\text { of } \\
\text { sherds }\end{array}$ \\
\hline A, floor. & & .. 23 \\
\hline C, floor. & & 26 \\
\hline C, fill... & & 73 \\
\hline B, floor. & & . 70 \\
\hline$\Lambda$, fill... & & .. 45 \\
\hline$B$, fill... & & .. 29 \\
\hline $\mathrm{F}$, floor. & & . 30 \\
\hline H, floor. & & 50 \\
\hline
\end{tabular}

Final Matrix

\begin{tabular}{|c|c|c|c|c|c|c|c|c|}
\hline Room........... & $\begin{array}{c}\lambda \\
\text { floor }\end{array}$ & $\begin{array}{c}\text { C } \\
\text { floor }\end{array}$ & $\begin{array}{c}\text { C } \\
\text { fill }\end{array}$ & $\begin{array}{c}\text { B } \\
\text { floor }\end{array}$ & $\begin{array}{l}\text { A } \\
\text { fill }\end{array}$ & $\begin{array}{l}\text { B } \\
\text { fill }\end{array}$ & $\begin{array}{l}F \\
\text { floor }\end{array}$ & $\begin{array}{l}\mathrm{H} \\
\text { floor }\end{array}$ \\
\hline A, floor. & - & 134 & 140 & 111 & 100 & 76 & 61 & 112 \\
\hline C, floor. & 134 & - & 174 & 174 & 143 & 129 & 117 & 119 \\
\hline C, fill... & 140 & 174 & - & 164 & 154 & 129 & 111 & 99 \\
\hline B, floor.... & 111 & 174 & 164 & - & 140 & 132 & 120 & 98 \\
\hline$A$, fill... & 100 & 143 & 154 & 140 & - & 174 & 153 & 97 \\
\hline$B$, fill $\ldots \ldots \ldots$ & 76 & 129 & 129 & 132 & 174 & - & 149 & 86 \\
\hline$F$, floor .......... & 61 & 117 & 111 & 120 & 153 & 149 & - & 118 \\
\hline $\mathrm{H}$, floor........... & 112 & 119 & 99 & 98 & 97 & 86 & 118 & \\
\hline
\end{tabular}

As can be seen, the sample from Room $\mathrm{H}$, floor is more similar in the assemblage of painted wares to samples at the ends of the represented time range than it is to any of the middle material. This may be because it is a "mixed sample." This would be the case if H, floor had been occupied throughout the time period represented, and if, in addition, more pottery had been broken and trampled into the floor during both early and late times than during the middle period. 
As can be seen from figure $46, b$, where the relationships between the samples are presented graphically, all but $H$, floor are related in a more or less linear temporal manner, but $\mathrm{H}$, floor is a "flyer," and the reasons for its differences from the other samples must be due to something other than chronologically based change in an isolated cultural assemblage, if the seriation is correct. However, the samples are so small that speculation on this point based on the seriation alone may lead to fallacious conclusions. It is worth mentioning that if more than one sample like $\mathrm{H}$, floor had been included in the seriation. seriating the data would have been well-nigh impossible.

Figure 48, c, d, shows the increase of Kiatuthlanna and Tularosa Black-on-White through time, the decline of Reserve Black-on-White from a large percentage of the total painted ware assemblage (neglecting $H$, floor), and the increase and subsequent decline of Snowflake Blackon-White and, to a lesser extent, Wingate Black-on-Red. It is interesting that the direction of change in popularity is diametrically opposed between Snowflake and Wingate, at least until the sample from F, floor is reached. I am tempted to speculate on very tenuous grounds again. If at least two clans occupied this site during the represented time range, and if they differed in the manufacture of pottery so that one clan made a higher percentage of Snowflake Black-on-White in proportion to Wingate Black-on-Red than the others, then perhaps alternating clan dominance would account for the appearance of this curve.

The sample from $H$, floor, as expected, behaves anomalously, the percentage of Reserve Black-on-White in particular being very high. Once again, Kiatuthlanna Black-on-White is present in a late setting.

The Hooper Ranch Pueblo.-This site afforded the most dubious seriation of any constructed with these materials. As can be seen from figure 46, c, the positions of samples in relation to one another scatter widely. Three samples, Kiva 3, floor, 5B, floor, and 3A, floor 2, bear little resemblance to the other samples, and little to each other. There is not a single pottery type in common between $3 \mathrm{~A}$, floor 2 and 5B, floor. As mentioned earlier. without the aid of the graphic presentation afforded by Chart 2 there would have been no indication of which sample should start the seriation, or in what direction it would proceed. Even the chart does not show the discrepancies between $3 \mathrm{~A}$. floor 2 and $5 \mathrm{~B}$, floor, and between them and the rest of the samples as well, as both the aforementioned samples actually cannot be placed on the chart. They have only been so placed for ease in comprehending the overall picture.

The seriation itself was based on 11 samples. Sample size and the final seriation matrix are shown below. 
TABLE 10.-SAMIPLE SIZE OF SHERIOS FROM HOOPER R.NCH PUEBI,O ANI FINAL, MATRIS

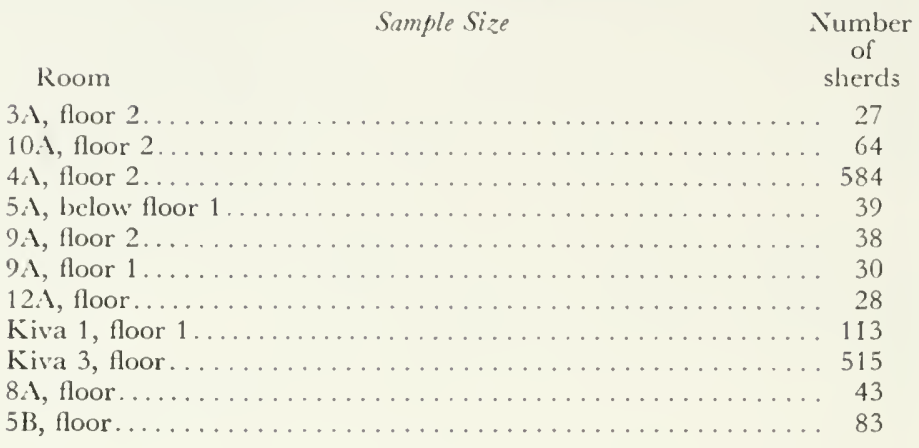

\section{Final Matrix}

\begin{tabular}{|c|c|c|c|c|c|c|c|c|c|c|c|}
\hline & $\begin{array}{c}3 . \\
\text { floor } \\
2\end{array}$ & $\begin{array}{c}10 . \mathrm{A} \\
\text { floor } \\
2\end{array}$ & $\begin{array}{c}4 \lambda \\
\text { floor } \\
2\end{array}$ & $\begin{array}{l}\text { below } \\
\text { floor } 1\end{array}$ & $\begin{array}{c}9 . \\
\text { floor } \\
2\end{array}$ & $\begin{array}{c}9 . \mathrm{A} \\
\text { floor } \\
\mathrm{I}\end{array}$ & floor & $\begin{array}{c}\text { Kiva } \\
1 \\
\text { floor }\end{array}$ & $\begin{array}{l}3 \\
1 \text { floor }\end{array}$ & $\begin{array}{r}8 \mathrm{~A} \\
\text { floo }\end{array}$ & $\begin{array}{r}5 \mathrm{~B} \\
\text { floor }\end{array}$ \\
\hline & - & 37 & & 42 & 49 & & 44 & 42 & 37 & & \\
\hline & 37 & - & 141 & 98 & 83 & 12 & 114 & 86 & 106 & & \\
\hline & 37 & 141 & - & 131 & 117 & & 37 & 117 & 108 & 105 & \\
\hline$r$ & 42 & 8 & 131 & - & 173 & 45 & 32 & 128 & 97 & & \\
\hline & 49 & 83 & 117 & 173 & - & 43 & 36 & 134 & 108 & & \\
\hline & 37 & 112 & & 145 & 143 & & 144 & 136 & 116 & 120 & \\
\hline & 4 & & & 132 & 136 & & & 165 & 138 & & \\
\hline & 42 & & & 128 & 134 & & & - & 129 & 0 & \\
\hline & 37 & 106 & & 97 & 108 & & & 129 & - & 125 & \\
\hline & 3 & 96 & & 93 & 94 & & & 130 & 125 & & \\
\hline & 0 & 4 & 42 & 27 & 21 & & & 39 & 32 & 4 & \\
\hline
\end{tabular}

The direction of the seriation is probably from 3A, floor 2 (early) to $5 \mathrm{~B}$, floor (late), as shown by the relative positions of floor 2 and floor 1 , Room $9 \mathrm{~A}$. It would not be wise to base any conclusions on the results of this seriation alone. Figure 49, $a-d$, shows the percentage of the total decorated ware sample constituted by each of the major types represented in the sample. The anomalous character of the samples of $3 \mathrm{~A}$, floor 2 and $5 \mathrm{~B}$, floor can be seen well. There is a sceming similarity between the assemblage of $3 \AA$, floor 2 , at the Hooper Ranch, and that of $\mathrm{H}$, floor, at the Rim Valley site (fig. 48, c, d). On this very tenuous basis the suggestion is ventured that there may be some sort of cultural similarity between the late settlers at Rim Valley and the early ones at the Hooper Ranch, and that the settlers of both sites during this common period of occupation, if such it be, belong to a tradition clivorced from that of the main settlement at either site. Still, it is best to remember that the data here presented are insufficient to warrant these speculations. Attention is also called to the fact that two sherds of Kiatuthlanna Black-on-White 


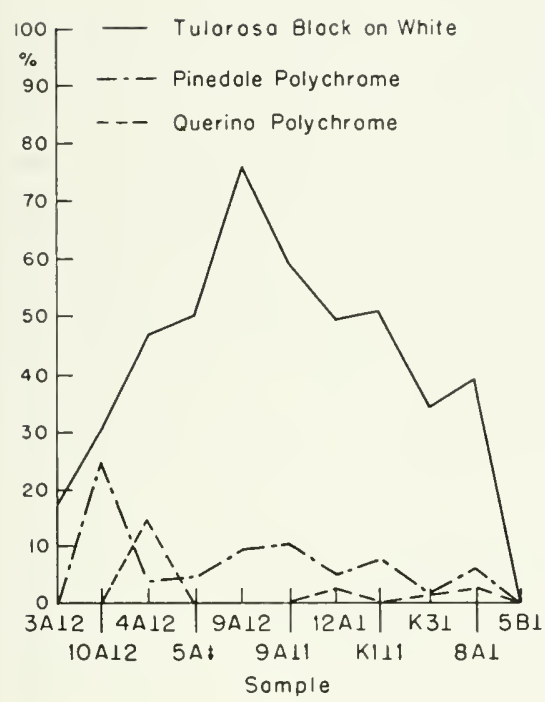

(a)

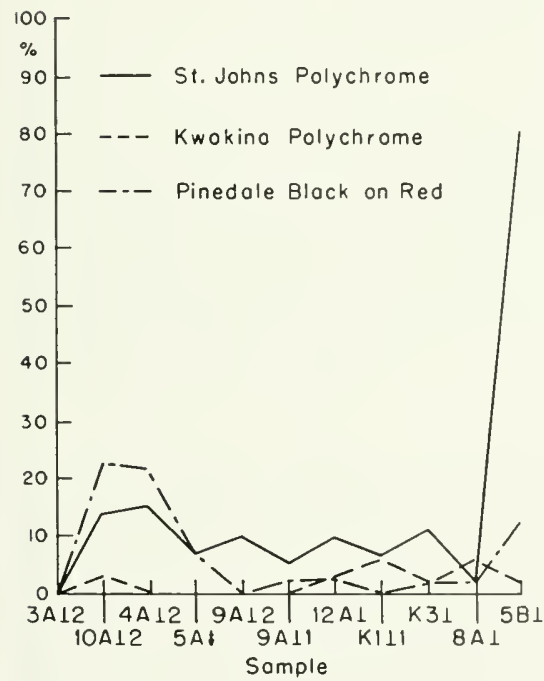

(c)

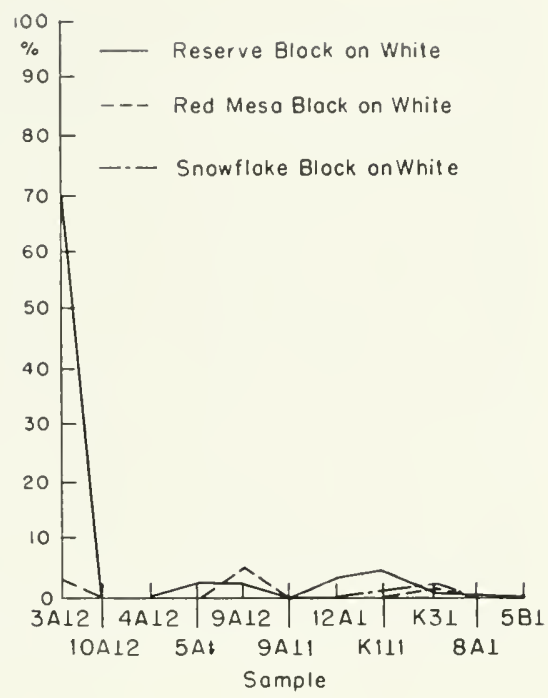

(b)

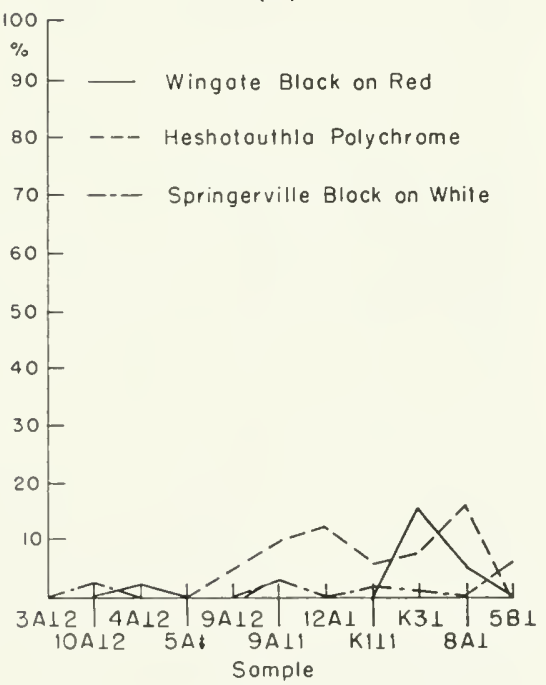

(d)

$1=$ Floor

$t=$ Below Floor

$\mathrm{K}=$ Kivo

$3 A \perp 2=$ Room $3 A$, Floor 2

K111 = Kivo 1, Floor 1

Fig. 49. Trends in painted pottery types illustrated by samples from Hooper Ranch Pueblo. 
(not shown on the graph) were found on Kiva 1 floor, and two on Kiva 3 floor. This may be accidental, but it also suggests an earlier provenience for the materials in those samples than is indicated in the seriation.

Evidently Tularosa Black-on-White was the dominant ware at the Hooper Ranch Site, and it seems to have increased in popularity until the terminal occupation of Room 9, floor 2: then it decreased. Wingate Black-on-Red has a late "rogue" and then decreases, being at its highest peak of popularity earlier than Heshota-uthla Polychrome, which does not reach its peak until we reach the material from the floor of Room 8A. Both types have disappeared from the floor of Room 5B. Room 5B is an anomaly, much elifferent in cultural content from the rest of the rooms. In it, St. Johns Polychrome has taken the dominant position held by Tularosa Black-on-White in the rest of the excavated materials. Room 5B, floor gives the appearance of being a mixed sample, if the rest of the seriated materials are in their proper place. The "pottery popularity" curves make it seem more probable that the seriation is not entirely correct, as their fluctuations have less the appearance of normal curves than is the case on the other sites.

\section{CONCLLSIONS}

(A) The site materials studied in this section seem to fall into three distinct groups, each with some temporal duration, to which I shall refer as periods 1 through 3. The earliest period seems to be that represented by the materials from the Goesling Site. This period is characterized, in the seriated material, by 80-92 per cent of Red Mesa Black-on-White, from 7-19 per cent of Kiatuthlanna Black-on-White, and a trace of White Mound Black-on-White.

Period 2, represented by the materials from Rhoton. Chilcott, and Thode, is much different in painted ware assemblage from the first. At Chilcott, Snowflake Black-on-White has appeared and dominates the painted wares, becoming more popular while Reserve Black-on-White becomes less popular, until at last Snowflake Black-on-White makes up 90 per cent of all the painted wares. Tularosa is ever present, but only from 2 to 9 per cent of the total painted wares. Kiatuthlanna Black-onWhite appears early, climbs to 11 per cent and then disappears. Red Mesa Black-on-White constitutes a small but increasing percentage (to 6 per cent of total painted wares) until the end of our represented period.

Though Rim Valley is different from the earlier sites, it bears some remarkable similarities to the Chilcott Site. The earlier portion of the Rim Valley Site shows a quite similar increase of Snowflake and decrease 
of Reserve, though Snowflake does not seem to have attained the popularity at Rim Valley that it had at Chilcott. The outstanding differences in the excavated collections from the Rim Valley and Chilcott sites seem to be three in number. First, Tularosa Black-on-White makes up a high percentage of the painted wares of Rim Valley quite "early," and continues to increase in popularity through time. Second, there is between 5 and 20 per cent of Wingate Black-on-Red until quite "late" at Rim Valley. Third, the late portion of the seriated materials from Rim Valley shows the decline in popularity of Snowflake Black-on-White ware. Rim Valley seems to me assignable in part to Period 2, in part to Early Period 3, both parts showing new cultural influences.

There may be a cultural stage temporally intermediate between Rim Valley and Hooper, as mentioned in the discussion of the Hooper Ranch seriation, and during which both Hooper and Rim Valley were occupied at more or less the same time. However, the evidence for this, from the floor of Room $\mathrm{H}$ at Rim Valley, and floor 2, Room 3A, at Hooper, is dubious.

At the Hooper Ranch Site, which represents Period 3, the excavated material is distinguished from the previous materials by the appearance of late types of painted wares, among them Heshota-uthla Polychrome, Pinedale Polychrome and Black-on-Red, and St.Johns Polychrome. Tularosa Black-on-White increases in popularity until Room 9, floor 2, and from then onward it declines. The presence of both early and late materials in the Hooper Ranch collections suggests that the duration of occupation represented by the Hooper materials is a long one in comparison with the materials from the other sites. The presence of Red Mesa in this context seems strange.

I strongly question the fixing of Room 5B, floor as "late" at the Hooper Ranch. Although the seriation "works" best with Room 5B, floor in that position, the sample is so anomalous that were the sample from Room 3A, floor 2 not included in the seriation, Room 5B, floor would scriate equally well at either the late or the early end of the sequence. I am less inclined to doubt the early position of Room $3 \mathrm{~A}$, floor 2, though there are good grounds for so doing. Chief among the reasons it has been included is its resemblance to Room $H$, floor, at Rim Valley.

If I had no more than the clata included in this chaper, I should be inclined to derive from them an initial cultural phase represented by the Goesling Ranch Site; a "gap" in the cultural record; and a new phase represented by the materials from Chilcott. The early portion of Rim Valley would then seem to me to be a blend of the Chilcott and another 
phase. including an increasing amount of Tularosa Black-on-White and the continuation of Wingate Black-on-Red in the painted ware assemblage. I should be tempted to see the further development of the Rim Valley phase in the early materials at Hooper Ranch, and either the development or adoption of a number of late painted wares there. After the "abandonment" of Rim Valley, I should be tempted to postulate the intrusion into the Rim Valley-Hooper area of a group of "foreigners" with a distinct material culture and their subsequent withdrawal or amalgamation with natives of the area.

These speculations are, of course, not seriously offered as any kind of culture-historical scheme for the area. I realize that most of them may well prove totally erroneous, but their presentation may enable the visualization of further problems, or corroboration of results from other lines of research.

(B) From the position of the samples from Room 3, fill, at Chilcott (25 sherds). Room F. floor (30 sherds) and Room H. floor (50 sherds), at Rim Valley, and Room 3A. floor 2, at Hooper (27 sherds) in the seriation, it is obvious that such samples are not suited to the RobinsonBrainerd seriation method. A swift glance at the Hooper Ranch seriation will show that even larger samples don't always seriate well with this method. This is probably because such samples have been accumulated over long periods of time.

Because of the unsatisfactory nature of some of our samples and the limitations of the method, I again stress two statements previously made. First, no inference made above applies to any site as a whole. These statements apply only to the materials from which they are drawn. Second, the evidence from the seriation is to be taken as suggestive, and in some cases, it is hoped, corroborative, but never as conclusive. However, I do feel that techniques like the Robinson-Brainerd seriation method are now and will increasingly become of utility in studies like the present one. I hope I have demonstrated some of the utilitarian aspects of such methods in archaeological analysis, while realizing still more that I have demonstrated some of their limitations. I have been encouraged rather than discouraged by the results of the applications of the Robinson-Brainerd method to our data. The method should yield much more dependable results when applied to large random samples of surface materials or to large scale excavations. 


\title{
V. An Analysis of Pottery Design Elements, Indicating Possible Relationships Between Three Decorated Types
}

\author{
By Constance Cronin \\ Research Assistant, Department of Anthropology \\ University of Chicago
}

During the summer of 1960 members of the archaeological staff of Chicago Natural History Museum excavated seven sites in the Little Colorado drainage of eastern Arizona. When the pottery was classified in the field. five established types were separated-Kiatuthlanna Blackon-White. Red Mesa Black-on-White, Reserve Black-on-White, Tularosa Black-on-White and Snowflake Black-on-White. The first four types have been relatively well studied, but Snowflake Black-on-White was less well known. It was first named by W. and H. S. Gladwin (1934, p. 22) and described by Colton (1941, pp. 62-63): however, it had never been completely analyzed from a large sample and the descriptions tended to be too general for use in a detailed study of relationships with other pottery types.

The present study was undertaken in an effort to analyze the constituent elements of the designs found on Snowflake Black-on-White pottery and also to inquire into the degree of relationship among the five types. The basic factor chosen for analysis was the design element. Each sherd was classified by its design element, and in this way small discrete units were separated and then recombined so as to reveal basic units and groups of designs which enable the worker in this field to recognize any one pottery type as a distinctive entity and which set it off from all other pottery types. Somewhat similar but not identical studies had been undertaken in the past. Beals, Brainerd and Smith (1945, pp. 87-137) compared Kana-a, Black Mesa, Sosi and other pottery type designs (but not elements) from a series of sites. Martin (1939, pp. 431445) studied, by element, Abajo Red-on-Orange as compared with La Plata Black-on-Orange. Therefore the present project was a pilot study to ascertain the lineage of Snowflake Black-on-White pottery, and its 
subsequent growth through time, thereby adding to the general fund of theory concerning the relationships and changes of pottery types in any given area. Since so much archaeological reconstruction relies heavily on ceramies, not only in the Southwest but also in Central and South America and the Near East, systematic studies are vital as aids in these reconstructions.

A cursory study of the sherds in the field suggested that Snowflake Black-on-White might have evolved from earlier ceramic types, perhaps specifically from Kiatuthlanna Black-on-White and Red Mesa Black-onWhite.

A superficial inspection of illustrations of sherds and whole pieces of Kana-a Black-on-White and Black Mesa Black-on-White suggested that these types might also have influenced the development of Snowflake Black-on-White. One should bear in mind, however, that the Snowflake Black-on-White found by the Museum staff was nearly always associated with Kiatuthlanna and Red Mesa Black-on-White.

The work was divided into two phases: the first, undertaken by Walter Boyer, formerly a ceramic restorer at the Museum, and myself, involved the actual sorting and setting up of the inventory of design elements present in all five pottery types; the second, that of interpreting the results, was primarily my own work, but a number of people aided me greatly by their criticisms and advice. This group includes Dr. Paul S. Martin, Dr. John B. Rinaldo, Dr. Elaine A. Bluhm, Mr. William A. Longacre and especially Dr. Arthur J. Jelinek, all of whom have my heartfelt thanks.

This paper follows the two-fold division used in the study itself.

\section{SORTING}

The 2188 sherds were sorted on the basis of design elements only, regardless of type. The elements were delineated as each new combination was observed for the first time. No attempt was made to fit a new and slightly different element into an existing category, for it was felt that some of these apparently minor differences might become important when the changes in one pottery type were followed through time. In this way, 45 categories of design elements were finally separated.

The sherds were then re-sorted into types by sites. Thus the Rim Valley group was sorted into "Kiatuthlanna at Rim Valley," "Snowflake at Rim Valley" and "Red Mesa at Rim Valley." This sorting was done by using the generally accepted criteria for pottery type recognition: presence or absence of slip; whiteness of slip; intensity of paint; temper; 
proportion of black to white; fineness and blockiness of elements; positioning of design on a pot; and the general "feel" of the total design and technique of manufacture on each sherd.

The last step in the sorting process subdivided each of the groups by design elements of pottery types at sites. Each of the numerous groups then contained " $\mathrm{X}$ design element of $\mathrm{Y}$ type at $\mathrm{Z}$ site." The chart (Table 11) of total design elements, prepared after the first step, was utilized here so that the number of elements remained the same.

\section{ANALYSIS}

In order to regularize the vast amount of data and the numerous variables in this study, a series of graphs and bar charts was utilized. These suggested a closer relationship between Kiatuthlanna and Snowflake than between Red Mesa and Snowflake, as had first been conjectured, and clearly showed that the relationship between these types was much closer at earlier sites than at the later sites, since fewer design elements were shared through time. (Since the Tularosa and Reserve types proved too divergent in elements shared with the other three types, we decided to omit them from the analysis. Our main concern was with establishing relationships, and these two types, though perhaps related to the rest, were sufficiently dissimilar to justify exclusion.)

To clarify further the relationships between Kiatuthlanna, Red Mesa and Snowflake, the data were quantified by applying the BrainerdRobinson method (Brainerd, 1951; Robinson, 1951) to the percentages of each design element, both for each type and for each site (Tables 12 and 13). (For an explanation of the Brainerd-Robinson method, see p. 87.) Two series of coefficients of similarity were arrived at, one of which showed degree of similarity within types at different time levels: Red Mesa at Chileott 1, with Red Mesa at the earlier site, Goesling, and at the later site, Chileott 2 (Table 12). These figures confirmed and agreed with our previous impressions regarding relationships between types and also revealed some additional unexpected correlations which are set forth below.

\section{CONCLUSIONS}

It is apparent that no firm conclusions can be drawn from this study, but some possible correlations can be suggested on both a specific and a general theoretical level.

By the use of the processes described above, our initial hypothesis concerning the relationships between Red Mesa and Snowflake was not 
confirmed-Red Mesa and Snowflake are not closer to each other than either is to Kiatuthlanna-but a different relationship is apparent (Table 13). In the earliest site (Goesling) Snowflake Black-on-White is probably not present and the coefficient of similarity between Kiatuthlanna and Red Mesa is 121.2, pointing out a definite but not extremely close relationship between the two types. In the next later site (Chilcott 1) Snowflake appears for the first time, along with Kiatuthlanna and Red Mesa. Here the similarity between Kiatuthlanna and Red Mesa has dropped to 94.4 and the relationship of Kiatuthlanna to Snowflake is 137.6, while the ratio of Red Mesa to Snowflake is 98.0. At this time level, then, Snowflake is closer to Kiatuthlanna than Red Mesa is to Kiatuthlanna or to Snowflake.

At Rim Valley, another site where all three pottery types occur together but at a later time level than at Chilcott 1, the figures (Table 13) reveal greatly attenuated relationships between the three types. The relationship between Kiatuthlanna and Red Mesa has dropped to 57.0, that of Kiatuthlanna and Snowflake has decreased to 97.9, and the Red Mesa-Snowflake ratio is now 68.1. The decreasing degree of closeness of all three types to each other is clearly evident here, but the figures also show that Snowflake is still closer to Kiatuthlanna than it is to Red Mesa or Red Mesa to Kiatuthlanna.

One might postulate that Snowflake began as a 2-1 blend of Kiatuthlanna and Red Mesa design elements not long after Red Mesa had grown out of Kiatuthlanna. It is perhaps naive to say that pottery types change through time but the question here is, how do they change? If we can demonstrate that one type came about as the result of a blending of manufacturing principles of two existing types, then we should be able to demonstrate the "drift" of each. The concept of linguistic drift was formulated by Sapir (1921) but it may be equally applicable here. An initial unity between Kiatuthlanna as the parent and Red Mesa and Snowflake as daughter types does not necessarily imply the same line of development for each through time. And, indeed, this study shows that the very close similarity of types near the time of origin may give way to quite individual and specific evolutionary lines as stylistic trends impel each type in a different direction from its source.

Even if the suggestion given above is true, we still expected to find the same regular though decreasing set of relationships of a type through time as we found between types at a site. For instance, if the coefficient of similarity of Kiatuthlanna to Snowflake at Chilcott 1 was 137.6, we assumed that the coeflicient of similarity of Snowflake at Chilcott 1 
and Snowflake at Chilcott 2 would be higher, since a type should be closer to itself than it is to another type. But such is not the case, for generally speaking there is a greater degree of similarity (shared design elements) between types at one site (e.g., Kiatuthlanna and Snowflake at Chilcott 1) than between different time levels of one type (e.g., Kiatuthlanna at Chilcott 1 and Kiatuthlanna at Rim Valley). Red Mesa in particular exhibits an extremely erratic course, jumping from 76.8 at Goesling to 107.5 at Chilcott 1 and then back down to 67.9 at Chilcott 2 . Since any coefficient of similarity below 100 is all but meaningless for showing relationships, are we to assume that Red Mesa is more Red Mesa at Chilcott 1 than it is at the other sites? Snowflake is more regular in its passage through time, but even here the divergence at the latest time period, Rim Valley, is greater than its similarity at the point of origin, Chilcott 1. Kiatuthlanna is difficult to assess here since it is found at only three sites, but even so the figures seem meaningful, since the extremely low coefficient of similarity is not what one would expect to find in one pottery type.

With respect to the design elements themselves, several trends are apparent in our limited sample both with regard to uses of elements in the decoration of the three types, and with respect to the overall distribution of the elements through time.

The observations for the three types are as follows:

Kiatuthlanna Black-on-IThite.-Fine line and checkerboard decoration occurred in all samples and the distribution of ticked fine lines and ticked triangles suggests continuous use throughout the distribution of this type in this study.

Earlier Kiatuthlanna seems to have been characterized by the use of squiggle-hatched elements and triangular solids.

Later Kiatuthlanna was apparently characterized by the use of wider lines, diagonal hatching or none at all, sawtoothed solids, and the rectangular scroll. Absent elements include squiggle lines and spirals and medium line.

Red Mesa Black-on-IWhite. -Fine line decoration is common to all samples and the distribution suggests that checker elements were also universally used, as were spirals and ticking. There appears to be a trend of less frequent usage of fine lines in combination with solid elements through time.

Squiggle lines characterize earlier Red Mesa, but no squiggle hatching was encountered. 
Later Red Mesa is characterized by the use of sawtoothed solids, greater use of solids in general, and diagonal hatching.

Snouflake Black-on-While.-Wide line and opposed solids are in frequent use through the whole range of our samples. The distribution suggests that fine line and solids were used through the whole range.

Early Snowflake seems characterized by the use of squiggle hatching and perhaps checkered elements.

Later Snowflake makes use of diagonal hatching and occasionally ticking, checker and spiral-scroll elements.

Thus we can see that the methodology used in this study is a valuable technique both to set out a detailed pottery type description and to trace genetic relationships between types. This method lends itself to both graphic and statistical presentation and may prove a useful aid in future ceramic research.

This study was undertaken in an attempt to delineate intertype relationships and also to discover the worth of this technique for further expanded studies. The latter aim we feel has been well demonstrated and we can only hope that the results of and the questions raised by the first stated purpose will be followed up and expanded by other workers in this field.

\section{NAMES OF DESIGN ELEMENTS REFERRED TO IN TABLE 11}

no.

Wide Line . . . . . . . . . . . . . . . . . . . . . 3

Medium Line . . . . . . . . . . . . . . . . . . . 2

Fine Line . . . . . . . . . . . . . . . . . . . . . 1

Fine Line and Solids . . . . . . . . . . . . . . . . . 9

Fine Line and Triangles . . . . . . . . . . . . . . . . . . . . . . 8

Fine Line and Ticked Triangles . . . . . . . . . . . . . . . . . 10

Line and Dot . . . . . . . . . . . . . . . . 6

line and Single Terrace . . . . . . . . . . . . . . . 21

Single and Double Ticked Lines . . . . . . . . . . . . . . . . . 7

Single and Double Ticked Lines and Ticked Triangle and Solids . . . . . . . 4

1 The sample from Chilcott 1 Site is peculiar in missing a number of clements common to Red Mesa at the Goesling and Thode Sites. These absent elements include ticking, checker and squiggle lines and suggest that the Chilcott 1 sample may be later than indicated by other means or perhaps indicates that several trends were initiated here. These would include the use of diagonal hatching on Kiatuthlanna anel Red Mesa while squiggle-hatching distinguished Snowflake. Other techniques unique here include complete lack of use of ticked elements on all types and the only instance of the use of opposed solids on Kiatuthlanna. 


\section{NAMES OF DESIGN ELEMENTS}

(continued)

Code

no.

Ticked Linc and Solids and Plain Line . . . . . . . . . . . . . . . . . 28

Ticked Line and Triangle and Plain Linc . . . . . . . . . . . . . . . 14

Squiggle Line . . . . . . . . . . . . . . . . . . . . . . . . . 20

Squiggle Hatch . . . . . . . . . . . . . . . . . . . . . . . 37

Squiggle Hatch and Line . . . . . . . . . . . . . . . . . . . . . . 39

Squiggle Hatch and Narrow Bands . . . . . . . . . . . . . . . . . . . . 25

Squiggle Hatch and Curvilinear Bands . . . . . . . . . . . . . . . . 19

Squiggle Hatch and Angular Bands . . . . . . . . . . . . . . . . . 26

Squiggle Hatch and Solid . . . . . . . . . . . . . . . . . . . . . . . . 38

Diagonal Hatch and Solid . . . . . . . . . . . . . . . . . . . 43

Diagonal Hatch Fragment. . . . . . . . . . . . . . . . . . . . . . . . 35

Intcrlocking Spiral . . . . . . . . . . . . . . . . . . . . . . . 32

Simple Spiral and Circle and Curved Fragment . . . . . . . . . . . 34

Solid and Hatch Scrolls . . . . . . . . . . . . . . . . . . . . . . . 30

Rectangular Scroll . . . . . . . . . . . . . . . . 42

Triangular Scroll . . . . . . . . . . . . . . . . . 40

Interlocking Rectangular Scroll with Key Endings . . . . . . . . . . . . . 27

Interlocking Solid and Hatch Rectangular Scrolls and Solid Scrolls . . . . . . 36

Solid Triangle . . . . . . . . . . . . . . . . . . . . . 5

Hatch Triangle . . . . . . . . . . . . . . . . . . . 13

Ticked Triangle and Single Terrace . . . . . . . . . . . . . . . . 11

Ticked Triangle and Fragment . . . . . . . . . . . . . . . . . . . 12

Sawtooth Solids . . . . . . . . . . . . . . . . . 24

Opposed Solids . . . . . . . . . . . . . . . . . . . . . . . . 44

Opposed Solids and Squiggle Hatch . . . . . . . . . . . . . . . . . . 29

Opposing Solid Step Triangle . . . . . . . . . . . . . . . . . . . . . . 41

Opposed Solid and Hatch Step Triangle . . . . . . . . . . . . . . . . 31

Opposed Hatch and Solid Terraces . . . . . . . . . . . . . . . . . . . . 33

Negative Diamond . . . . . . . . . . . . . . . . . 22

Negative Parallelograms . . . . . . . . . . . . . . 23

Checkerboard . . . . . . . . . . . . . . 16

Dotted Checker . . . . . . . . . . . . . . . . 15

Diamond Checker . . . . . . . . . . . . . . . 17

Checker and Line . . . . . . . . . . . . . . . . . . . . . . . 18

Miscellaneous . . . . . . . . . . . . . . . . . 45 
TABLE 11. PERGENTAGES OF GIVEN TYPES BY 1)ESIGN ELEMENTS AT GIVEN SITES

\section{Goesling}

No. of sherds $360 \quad 554$ 1)esign no. ${ }^{1} \quad h \quad R .1 /$

$$
3
$$

$$
2
$$

1

9

8

10

6

21

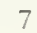

19

26

38

43

35

32

34

30

42

40

27

36

5

13

11

12

24

44

29

41

31

33

22

16

15

17

18

45
$.3 \quad .5$

$\begin{array}{rr}13.6 & 7.4\end{array}$

$\begin{array}{ll}12.7 & 22.2\end{array}$

$10.3 \quad 2.2$

$10.3 \quad 17.7$

$1.9 \quad 2.0$

.82 .0

$1.9 \quad 2.3$

$6.4 \quad .4$

1.1

$1.4 \quad 1.8$

$2.2 \quad 1.8$

$3.1 \quad 1.1$

$2.7 \quad .4$

$3.6 \quad .4$

$.8 \quad 1.3$

$1.9 \quad 1.8$

2.5

$.3 \quad .7$

$\begin{array}{ll}1.6 & 4.7\end{array}$

.52 .5

.5

$1.4 \quad 1.4$

$$
1.4 \quad 1.4
$$$$
1.6
$$$$
.5 \quad .5
$$$$
.9
$$$$
.8 \quad 4.5
$$$$
1.3
$$$$
1.4 \quad 1.1
$$$$
.4
$$

$\begin{array}{ll}1.6 & 2.7\end{array}$

$9.7 \quad 4.3$

$1.1 \quad .4$

$.3 \quad .9$

1.13 .8

\section{Chilcott 1}

$31.105 \quad 294$

$\begin{array}{lll}22.6 & 9.5 & 23.8\end{array}$

$\begin{array}{ll}5.7 & 3.1\end{array}$

$\begin{array}{lll}6.4 & 4.8 & .3\end{array}$

$10.5 \quad 2.7$

.7

$1.9 \quad .3$

$1.0 \quad .3$

.3

$\begin{array}{rrr} & 1.0 & .7 \\ .0 & 1.0 & 2.4\end{array}$

6.4

6.4

6.4

6.4

\section{8}

8.6

2.9

$\begin{array}{ll}3.8 & .3\end{array}$

$1.0 \quad .3$

.7

$1.0 \quad 1.7$

.3

$\begin{array}{lll}9.7 & 9.5 & 1.0\end{array}$

1.9

1.9

2.9

16.

3.8

3. 3

$\begin{array}{ll}1.0 & 1.0 \\ 3.8 & 1.0\end{array}$
Chilcott 2

$13 \quad 17$

R.M S

23.5

7.7

15.4

7.7

$5.3 \quad 6.6$

5.9

11.8

Chilcott 3

$19 \quad 15$

R.I S

$31.6 \quad 33.3$

26.3

5.3
5.9

$\begin{array}{ll}15.4 & 11.8\end{array}$

$15.4 \quad 6.6$

6.6

6.6

5.3

5.3

5.3

5.9

6.6

3.2

3.2

.3

$\begin{array}{lll}6.4 & 16.2 \quad 31.3\end{array}$

$23.1 \quad 11.8$

$10.5 \quad 33.3$

$1 /=$ Kiatuthlanna; $\quad R M=$ Red Mesa $; \quad S=$ Snowflake. 
TABLE 11. - PERCENTAGES OF GIVEN TYPES BY DESIGN ELEMENTS AT GIVEN SITES (continued)

\begin{tabular}{|c|c|c|c|c|c|c|c|}
\hline \multirow[b]{2}{*}{ No. of sherds } & \multicolumn{2}{|c|}{ Thode } & \multicolumn{3}{|c|}{ Rim Valley } & \multicolumn{2}{|c|}{ Rhoton } \\
\hline & 46 & 68 & 22 & 67 & 139 & 7 & 20 \\
\hline Design no. ${ }^{1}$ & $R M$ & $S$ & $h$ & $R M$ & $S$ & $R . M$ & $S$ \\
\hline 3 & & 39.7 & 9.1 & & 10.8 & 14.3 & 25.0 \\
\hline 2 & 19.6 & 7.3 & 9.1 & 31.3 & 9.3 & & \\
\hline 1 & 8.7 & & & 7.5 & & & \\
\hline 9 & & 1.5 & & 7.5 & 1.4 & & 5.0 \\
\hline 8 & 6.5 & & & 1.5 & & & \\
\hline 10 & & & 4.5 & & & 14.3 & \\
\hline 6 & 2.2 & & & 7.5 & 3.6 & & \\
\hline 21 & & 1.5 & & 1.5 & & & \\
\hline 7 & 4.3 & & & 1.5 & & & \\
\hline 4 & & & & & & & \\
\hline 28 & & & & & & & \\
\hline 14 & & & & & .7 & & \\
\hline 20 & 4.3 & & & 1.5 & & & \\
\hline 37 & & & & 1.5 & & & \\
\hline 39 & & & & & & & \\
\hline 25 & & & & & & & \\
\hline 19 & & & & & & & \\
\hline 26 & & & & & & & \\
\hline 38 & & 1.5 & & & & & \\
\hline 43 & 21.7 & & & & & 14.3 & 5.0 \\
\hline 35 & 4.3 & & & 4.5 & & & \\
\hline 32 & & & & 3.0 & 1.4 & & \\
\hline 34 & 4.3 & 2.9 & & 3.0 & & 28.6 & \\
\hline 30 & & & 4.5 & & .7 & & \\
\hline 42 & & & & 1.5 & 1.4 & & \\
\hline 40 & & & & 1.5 & 1.4 & & \\
\hline 27 & & & & & & & \\
\hline 36 & & & & 6.0 & .7 & & \\
\hline 5 & & 5.9 & & & & & \\
\hline 13 & & & & & & & \\
\hline 11 & & & & & & & \\
\hline 12 & & & & & 3.6 & & \\
\hline 24 & 2.2 & 1.5 & 4.5 & & 7.2 & & 10.0 \\
\hline 44 & 2.2 & 10.3 & & & & & 20.0 \\
\hline 29 & & & & & 29.5 & & \\
\hline 41 & & & & & & & \\
\hline 31 & 2.2 & & & & & & \\
\hline 33 & & 2.9 & & & .7 & & \\
\hline 22 & & .7 & & & .7 & & \\
\hline 16 & & & 4.5 & 1.5 & 1.4 & 28.6 & \\
\hline 15 & & 7.3 & 9.1 & 4.5 & 1.4 & & \\
\hline 17 & 2.2 & & 22.7 & & & & 5.0 \\
\hline 18 & & 16.1 & & & & & \\
\hline 45 & 15.2 & & 31.8 & 11.9 & 21.6 & & 30.0 \\
\hline
\end{tabular}


TABLE 12.-BRAINERD-ROBINSON RATIOS, SHOWING SIMILARITY WITHIN SINGLE TYPES AT SITES OF DIFFEREN'T HORIZONS

$\begin{array}{lccccccc}\text { Pottery Types } & \text { G-C1 } & \text { C1-C2 } & \text { C2-C3 } & \text { C3-T } & \text { T-RV } & \text { C1-RV } & \text { G-RV } \\ \text { Kiatuthlanna..... } & 37.0 & - & - & - & - & 44.0 & 17.6 \\ \text { Red Mesa...... } & 76.8 & 107.5 & 67.9 & 74.0 & 110.3 & - & - \\ \text { Snowflake........ } & - & 125.3 & 105.5 & 105.1 & 103.6 & - & -\end{array}$

TABLE 13.-BRAINERD-ROBINSON RATIOS, SHOWING SIMILARITY WITHIN POTTERY TYPES AT SINGLE SITES

\begin{tabular}{|c|c|c|c|c|c|c|c|}
\hline Pottery 'Types & G & $\mathrm{C} 1$ & $\mathrm{C} 2$ & C3 & $\mathrm{T}$ & $\mathrm{RV}$ & $\mathrm{R}$ \\
\hline $\begin{array}{l}\text { Kiatuthlanna- } \\
\text { Red Mesa. }\end{array}$ & 121.2 & 94.4 & - & - & - & 47.0 & 一 \\
\hline $\begin{array}{l}\text { Kiatuthlanna- } \\
\text { Snowflake... }\end{array}$ & - & 137.6 & - & - & - & 97.9 & - \\
\hline $\begin{array}{l}\text { Snowflake-Red } \\
\text { Mesa.......... }\end{array}$ & - & 98.0 & 62.4 & 105.6 & 63.8 & 67.7 & 38.5 \\
\hline
\end{tabular}

$\mathrm{G}=$ Goesling Site; $\mathrm{C} 1=$ Chilcott $1 ; \mathrm{C} 2=$ Chilcott 2; C3 = Chilcott $3 ; \mathrm{T}=$ Thode; $\mathrm{RV}=\mathrm{Rim}$ Valley; $\mathrm{R}=$ Rhoton. 


\title{
VI. Artifacts
}

\author{
By John B. Rinaldo \\ Associate Curator. Department of Anthropology \\ Chicago Vatural History. II useum
}

\section{INTRODUCTION}

The stone and bone artifacts were analyzed with the aim of tracing the cultural developments within the area of the upper Little Colorado and comparing them with developments in neighboring areas. Although we continued to be interested in the ways in which the artifacts were made and used, a comparison of artifact types by areas and horizons took precedence in this analysis. (For dimensions, proveniences and detailed descriptions of particular stone artifacts see Martin, Rinaldo, Longacre, and Freeman, 1961.)

Before A.D. 850 changes in the stone and bone artifacts were few and occurred at widely spaced intervals. After this time, change took place more rapidly and items such as stone axes which were not part of the native complex appeared as trait unit intrusions. Somewhat later we find the use of coursed stone masonry structures, black-on-white pottery and full-grooved axes.

Shells from the Pacific coast found at Hooper Ranch Pueblo (Martin, Rinaldo and Longacre. 1961, p. 108) indicate that there was trade between this area and the region to the south and west. Such traits as hachured black-on-white pottery, a crude sort of banded masonry. fullgrooved axes, and certain features of the Great Kiva (deflector and masonry-lined vaults) make it appear that there was also trade between the Little Colorado and the Chaco district to the north. This is borne out by the recovery, in the Chaco district, of shells and pottery which were probably traded from areas to the south and west (Judd, 1954, pp. $88-89,196)$.

\section{TOOLS USED IN THE PREPARATION}

\section{AND STORAGE OF FOOD}

There appears to have been a cultural lag between the upper Little Colorado and the neighboring areas to the south at about A.D. 300. 
Troughed metates and tabular wo-hand manos, which were present at the SU Site (Martin and Rinaldo, 1947, pp. 320, 328) and at the Bluff Site (Haury and Sayles, 1947, pp. 66, 67), were lacking at the Tumbleweed Canyon Site. The lack of pottery at the latter site may be another indication of the conservative nature of this culture.

\section{MaNos}

Over 140 manos or fragments thereof were removed from the eight excavated sites (including the three Chilcott sites). These have been classified into major groups according to the size and number of grinding surfaces, and into minor classes based on the shapes of the grinding surfaces in various combinations. A large number of fragments were recovered that were too small to classify.

One-hand Manos (fig. 50).- Thirty-two specimens were recovered. The majority of these are oval or sub-rectangular in outline (fig. 50). They range in length from 10.1 to $16.5 \mathrm{~cm}$., in width from 7.1 to $11.2 \mathrm{~cm}$., and in thickness from 3.2 to $8.6 \mathrm{~cm}$.

The manos in one sub-group are more sharply convex across the short dimension than across the longer one. On the few specimens where striae can be seen they cross the short dimension at an angle of about $80^{\circ}$ to the long axis. These appear to be more closely related to the manos of the Beach Sites such as Little Ortega Lake (Martin and Rinaldo, 1960a, p. 17, fig. $4, b$ ) than to those of the later sites. They are also more like those of the Cochise culture of southern Arizona, particularly Ventana Cave (Haury, 1950, p. 313), than like those of more closely neighboring Cochise sites such as Wet Leggett Site (Martin, Rinaldo and Anters, 1949. pp. 66-71) or the Cienega Site (Haury, 1957. p. 20). These manos came only from Tumbleweed Site. There were six specimens in the group and they are associated only with basin metates.

Another division comprises manos with convex or bluntly convex grinding surfaces. In two instances they are opposite a flat grinding surface. There are seven specimens in this group, most of which come from the Chilcott Sites (four specimens), although two came from the Thode Site and one from Rim Valley Pueblo. This sort of mano is usually more common on earlier sites both in this area (Martin and Rinaldo, 1960a. pp. 70-73) and at Forestdale (Haury, 1940, pp. 99-100: Haury and Sayles, 1947, p. 66), although a few have been recovered from later sites. These manos are more commonly associated with basin metates but were probably also used with trough metates.

A third sub-group of the smaller manos has flat grinding surfaces. Ninetcen specimens of this type were found, and it is thus the largest 

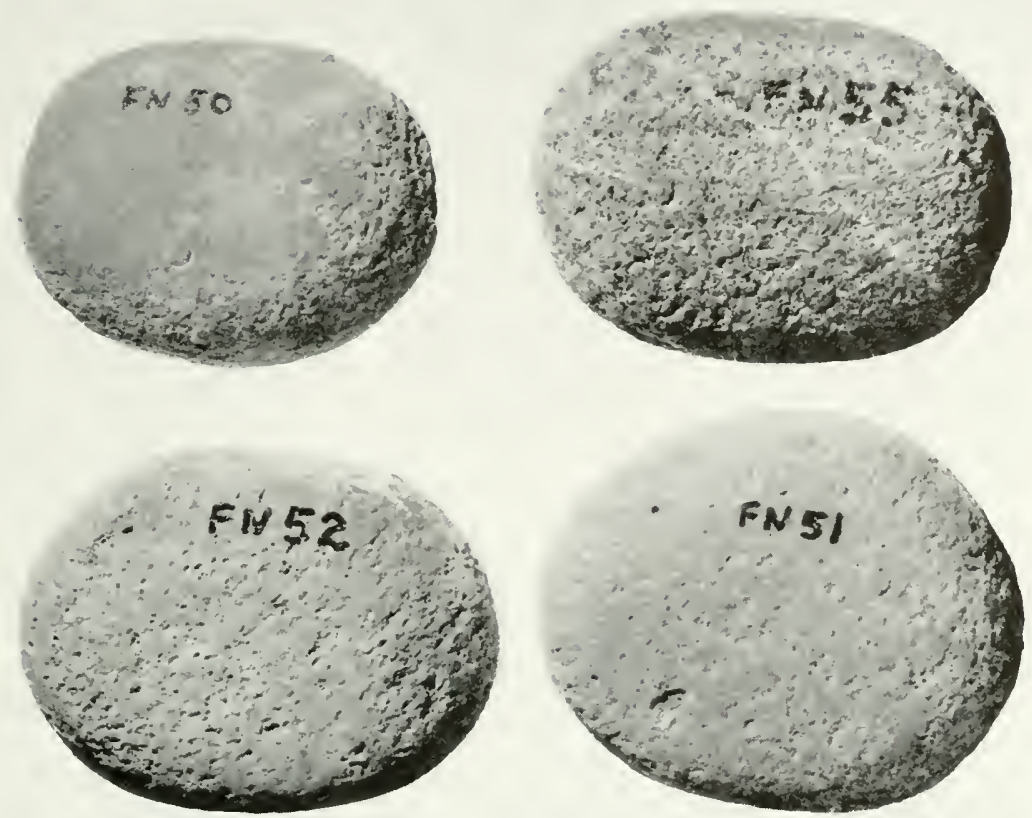

FIg. 50. One-hand manos, Tumbleweed Canyon Site. Length of lower right specimen, $13.4 \mathrm{~cm}$.

sub-group of shorter manos. They were recovered from most of the sites excavated, including the Tumbleweed Canyon Site on the early end of the sequence and Rim Valley Pueblo on the later end. Occasionally these manos are rectangular or sub-rectangular in outline. This variety is occasionally associated with trough type metates. Generally these manos appear to be a class which forms the link in the development into the longer flat-tabular mano and eventually into the beveled mano. They could have been used for a short time on a trough metate without being worn to a convex shape on the sides of the trough because they are short, but this type is believed to have been commonly associated with a flat metate.

Two-hand manos (fig. 51).- These longer manos comprise the larger of the two major groups and total 113 specimens. They range up to $25 \mathrm{~cm}$. in length (average length about $18 \mathrm{~cm}$.), and the width is about $11 \mathrm{~cm}$. Occasional specimens are $13.5 \mathrm{~cm}$. wide, and a few loaf-shaped speci- 

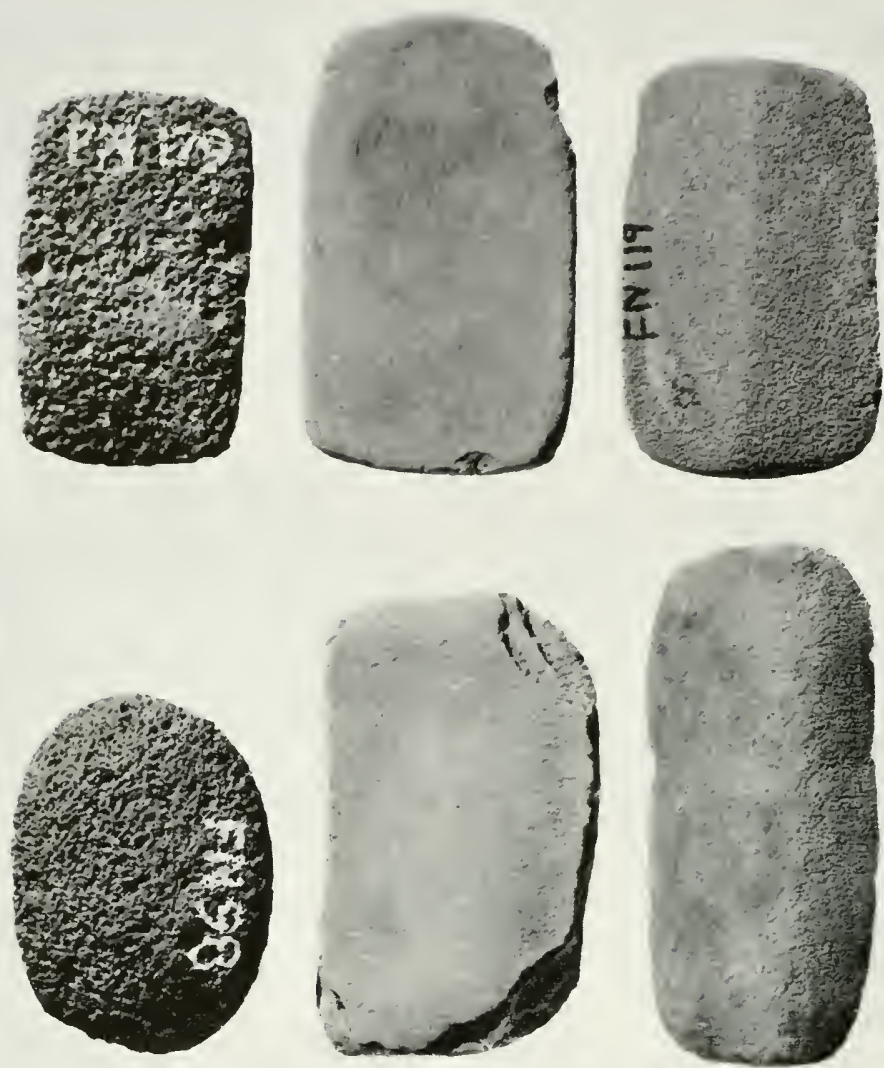

Fig. 51. Intermediate and late types of manos from Rim Valley Pueblo, Thode Site, and Chilcott Site 1. Length of lower right specimen, $25.0 \mathrm{~cm}$.

mens are over $5 \mathrm{~cm}$. thick. These larger manos were found in the same rooms with trough metates and slab metates. Only two longer specimens were found on the Tumbleweed Canyon Site, and this sort of mano occurs most often on later sites throughout the northern Southwest (Woodbury, 1954, p. 70). By comparison, one-hand manos have only a sporadic distribution on later sites. 
In this major grouping there were about 54 manos which had single grinding surfaces ranging from flat to convex in shape. Many of these were mere fragments. The majority of complete specimens are rectangular in outline, although many have rounded corners: they were probably used on trough metates.

A second minor group consists of those with grinding surfaces beveled into two fairly equal planes, but with no grinding surface on the opposite or upper side. There are sixteen specimens in this group, and (with two exceptions, one from the Hooper Ranch Pueblo, one from Chilcott Site 1) they come from the Rim Valley Pueblo. This distribution-primarily on the later sites-parallels the increase in popularity of this type in other areas such as the Jeddito (Woodbury, 1954, fig. 9. p. 82), the Rio Grande (Kidder, 1932, p. 71), and the Reserve area (Martin, et al., 1956, p. 58), during late Pueblo III and early Pueblo IV.

\section{Rubbing Stones}

(Figure 52)

None of these tools were shaped in outline. The 26 specimens recovered fall into two major groups (whether they have one rubbing surface or two rubbing surfaces opposite each other). A further division may be made on the somewhat arbitrary distinction between flat and slightly convex rubbing surfaces. No rubbing stones were recovered from the Chilcott Sites, and only one from the Great Kiva at Hooper Ranch Pueblo, although several were recovered from the secular rooms. A few have striations on their rubbing surfaces like the manos, and they may have been used as small manos.

Fourteen specimens have single rubbing surfaces. Most of these rubbing stones are oval in outline. They range in length from 4.0 to $11.3 \mathrm{~cm}$., in width from 3.0 to $10.0 \mathrm{~cm}$., and in thickness from 0.7 to $5.0 \mathrm{~cm}$. Six specimens of this group were recorered from the Tumbleweed Canyon Site and fewer from the later sites. Whether the diminished numbers of this particular group in late sites denote an actual decrease in popularity of this tool throughout the area in the later phases is not quite certain because ordinarily those with two rubbing surfaces are more frequent on the late sites. However, the greater frequency of rubbing stones at the Tumbleweed Canyon Site and their paucity at the Hooper Ranch Pueblo corroborates the theory that they are scarce on the latest sites, as was seen in the Reserve area (Martin, et al., 1956, p. 58; Rinaldo, 1959, p. 229).

The group with two rubbing surfaces comprises 12 specimens, 3 with flat rubbing surfaces and 9 with slightly convex ones (including one speci- 

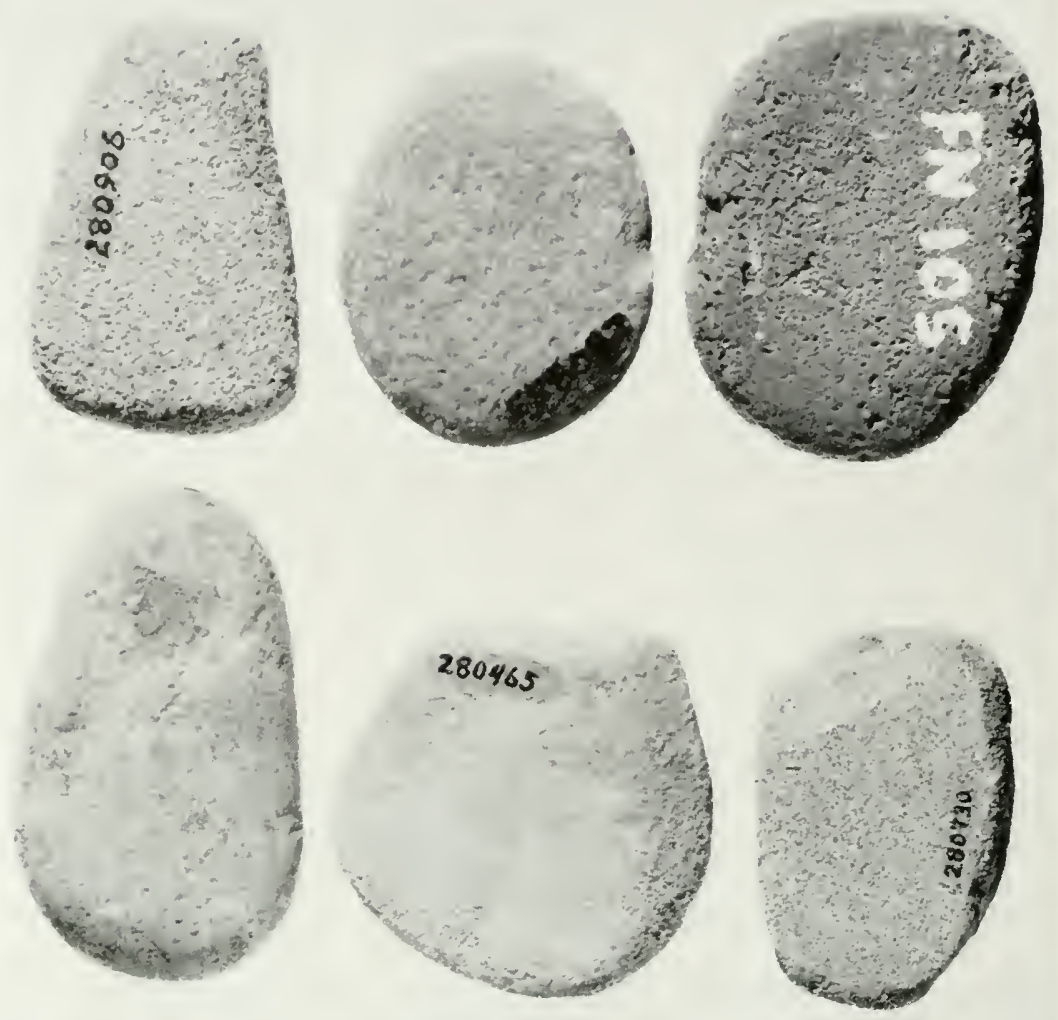

FIG. 52. Rubbing stones, miscellaneous types, from Goesling Site, Thode Site, Rim Valley Pueblo, and Hooper Ranch Pueblo. Length of lower right specimen. $5.8 \mathrm{~cm}$.

men with this shape of rubbing surface opposite a flat one). These were most common at the Thode Site and less popular both earlier and later. In general they are thinner than the rubbing stones with only one rubbing surface.

\section{Pestles}

(Figure 53, $a, b, e$ )

These clongate crushing tools are less common in the upper Little Colorado than they are in the Reserve area (Martin and Rinaldo, 1960a, p. 64) or farther south in the San Simon Branch of the Mogollon (Sayles, 
1945). Only four of these were recovered from three sites out of the eight tested. The pestles fall into three classes: cylindrical, pear-shaped, and multiface.

The cylindrical pestles are elongate, roughly cylindrical tools with tapering ends, the larger extremity battered from use. The larger speci-

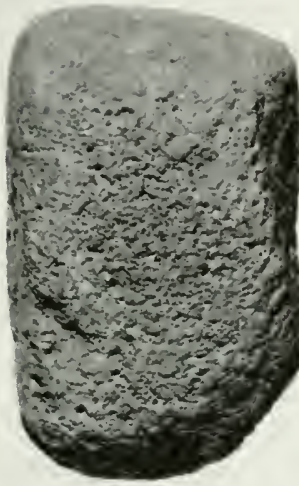

a

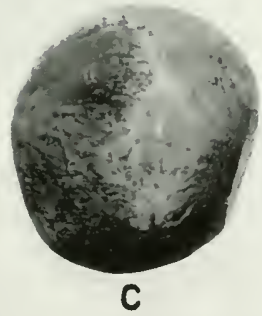

C

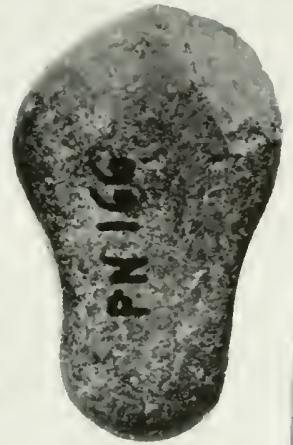

b

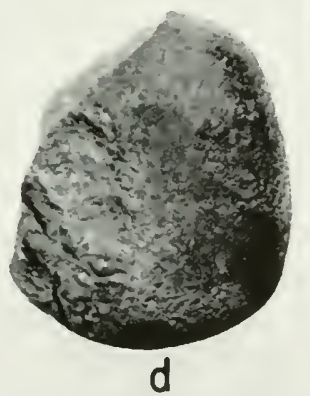

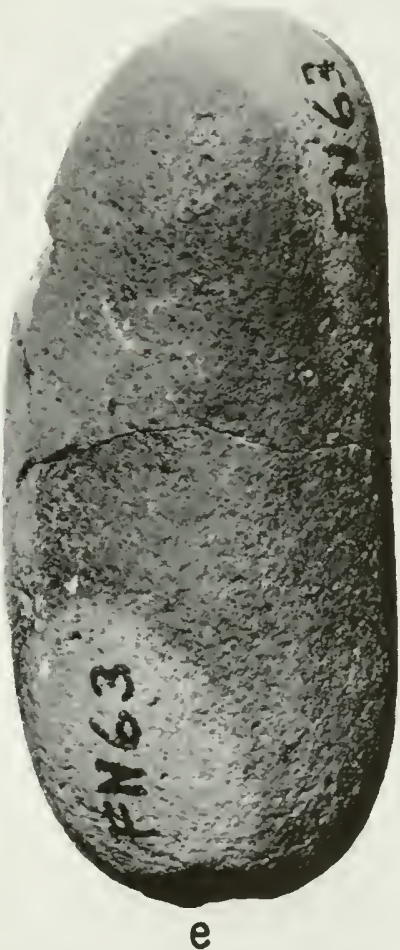

e

Fig. 53. Pestles, miscellaneous types, and hammerstones, Tumbleweed Canyon Site and Rim Valley Pueblo. Length of $e, 22.4 \mathrm{~cm}$.

men is $22.4 \mathrm{~cm}$. long and $10.2 \mathrm{~cm}$. in diameter, the smaller about $10 \mathrm{~cm}$. shorter and $1.0 \mathrm{~cm}$. less in diameter. The larger specimen comes from the Tumbleweed Canyon Site and is rery much like one from the SU Site (Martin and Rinaldo, 1940, p. 52, fig. 23) and also like one from Crooked Ridge Village (Wheat, 1954, p. 117, fig. 43, d). 
Another pestle is long and roughly pear-shaped, its smaller end roughened from use. It is $11.5 \mathrm{~cm}$. long, $7.0 \mathrm{~cm}$. wide, and $4.9 \mathrm{~cm}$. thick. It was recovered from Room B at Rim Valley Pueblo, a room which lacked a mortar or even a basin metate, but which was provided with three flour receptacles with stone slab bottoms. One of these slabs had been roughened and the pestle may have been used with it.

A multiface pestle came from the same room. Like many pestles of this type it has one end pitted and battered from use as a pestle and one broader surface striated from use in grinding. It could have been used in the same flour receptacle. Multiface pestles were recovered at Point of Pines (Wheat, 1954, p. 117, fig. 43, e) and in the Reserve area (Martin, Rinaldo, and Barter, 1957, p. 56).

\section{Metates}

(Figure 54)

The nether milling tools which were used with manos fall into four major groups within which some minor distinctions may be made. These are basin metates, trough metates with only one end of the trough open, through trough metates, and flat metates. Thirty-nine specimens were recovered, but most of these are fragments. We were able to distinguish between basin metates, trough metates, and slab metates in fragmentary form, but we could rarely distinguish between the two types of trough metates.

The basin metates (fig. 54, left) are with one exception (a fragment) from Tumbleweed Canyon Site. These are thick slabs of igneous rock, without regularity of outline, having a shallow basin in one broad surface. This basin is usually smooth in the center and pitted from pecking near the periphery. All of the specimens were broken but enough could be pieced together from the scattered fragments to give us a good idea of their original appearance but only a rough idea of actual dimensions. One of these that may or may not be typical was $32.0 \mathrm{~cm}$. long, 30.0 $\mathrm{cm}$. wide, and $2.6 \mathrm{~cm}$. thick. These are similar to the dimensions and shape of the "thin slab" basin metates from the Beach Sites (Martin and Rinaldo, 1960a, p. 24), and similar in form to those of the Chiricahua stage of the Cochise Culture (Sayles and Antevs, 1941, pl. 9). They are also similar to those of the Hilltop, Penasco and Pine Lawn Phases (Haury and Sayles, 1947, p. 64; Sayles, 1945, p. 50; Martin, 1943, p. 186), although trough metates were somewhat more popular during most of these "pithouse" phases.

A nearly complete specimen of trough metate with trough open at one end only was found at the Goesling Site (fig. 54, center). This metate 

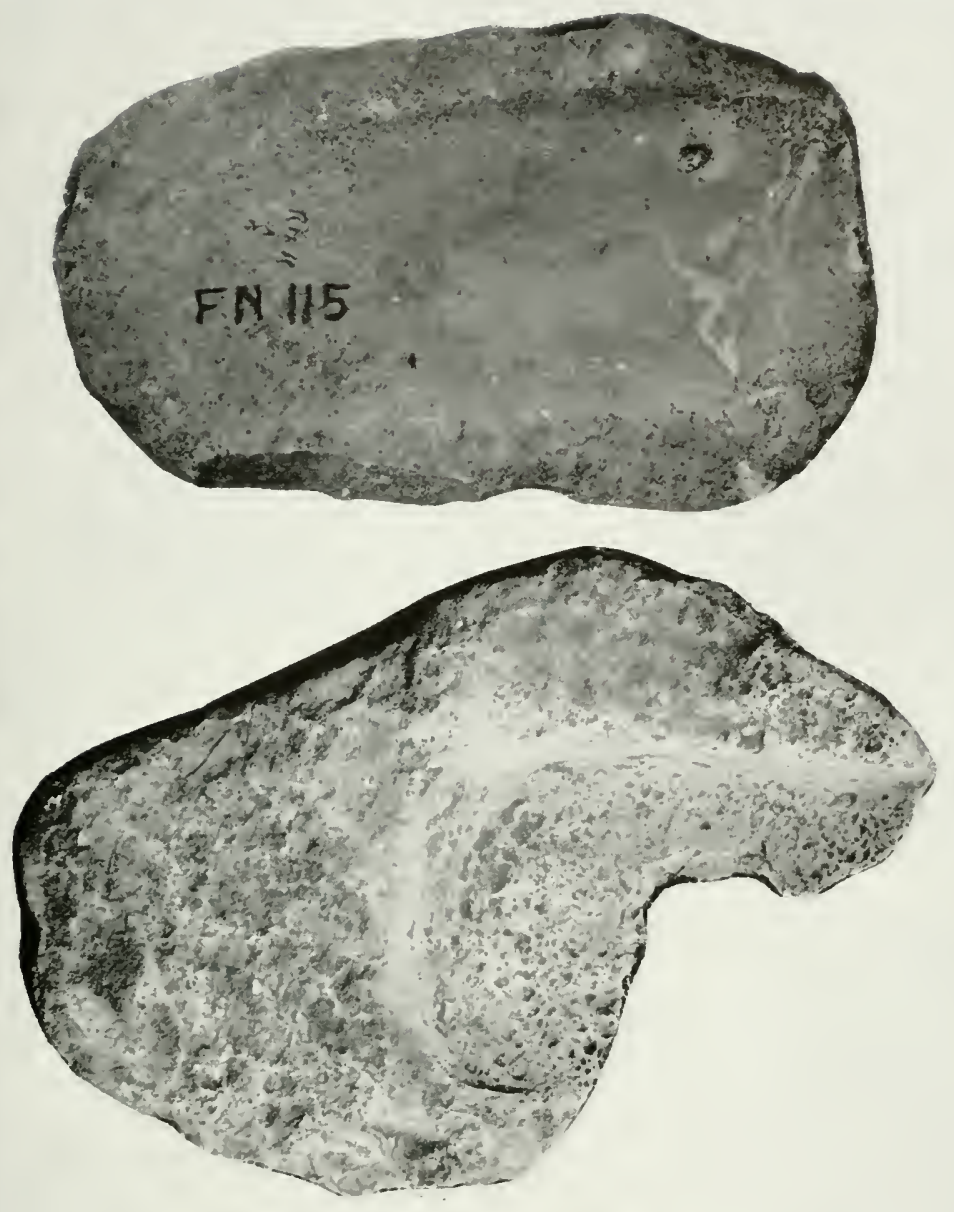

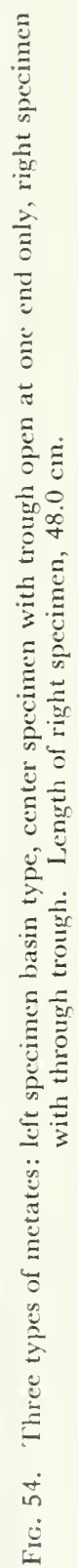


is macle from a generally oblong slab and has a flat shelf for the mano to rest on at the closed end. The sides of the trough are nearly straight, the bottom and sides of the shab rough hewn. Except in size (it is smaller) it is much like the metates of the Modified Basket Maker period (Roberts, 1940, p. 118; Martin, 1939. pp. 398-399), but also like those at the Twin Butte Site (Wendorf, 1953, p. 128).

Through trough metates (fig. 54, right) occurred at the Chilcott Sites and all the later sites in this area. These specimens are made from fairly thick oblong blocks and the trough runs from end to end, although in some supposedly little-used specimens it slopes up at the near end. One of the whole specimens (from the Rim Valley Pueblo) has the following dimensions: length, $43.5 \mathrm{~cm}$; width, $27.0 \mathrm{~cm}$; thickness, 8.5 $\mathrm{cm}$; width of trough, $22.5 \mathrm{~cm}$; depth of trough, $4.8 \mathrm{~cm}$. There were seven complete specimens recovered. They are comparable to metates from the neighboring areas and broadly similar ones are found throughout the Southwest (Bartlett, 1933, p. 23-27; Martin, et al., 1956, p. 73).

A few small fragments of slab metates were recovered from the later sites. They are too small for further identification or analysis, but are enough to indicate the presence of a type which was the predominant one at Table Rock Pueblo.

\section{Small Metate-Like Grinding Stones}

Three small metate-like grinding stones were recovered in Room A, Rim Valley Pueblo. One of these measures $27.0 \mathrm{~cm}$. long, $25 \mathrm{~cm}$. wide, and $7.5 \mathrm{~cm}$. thick, with a depressed grinding surface on one side approximately $12.0 \mathrm{~cm}$. in width and $0.5 \mathrm{~cm}$. in depth. These surfaces run the length of the stones as in trough metates, and the scratches they bear resulting from use run in this direction also.

These tools are similar to some of the smaller metates recovered at the Point of Pines site, Arizona W:10:51 (Wendorf, 1950, p. 54), and the grinding slabs from Ruin B, Nantack Village (Breternitz, 1959, p. 65). They were also recovered from sites in the Reserve area such as Higgins Flat Pueblo (Martin, et al., 1956, p. 78) and Three Pines Pueblo (Martin and Rinaldo, 1950b, p. 468).

\section{MORTARs}

(Figure 55)

The seven mortars found are with one exception stones with cupshaped depressions excavated into one surface. They range in length from 15.8 to $29.5 \mathrm{~cm}$., in width from 10.6 to $19.0 \mathrm{~cm}$., and in thickness 


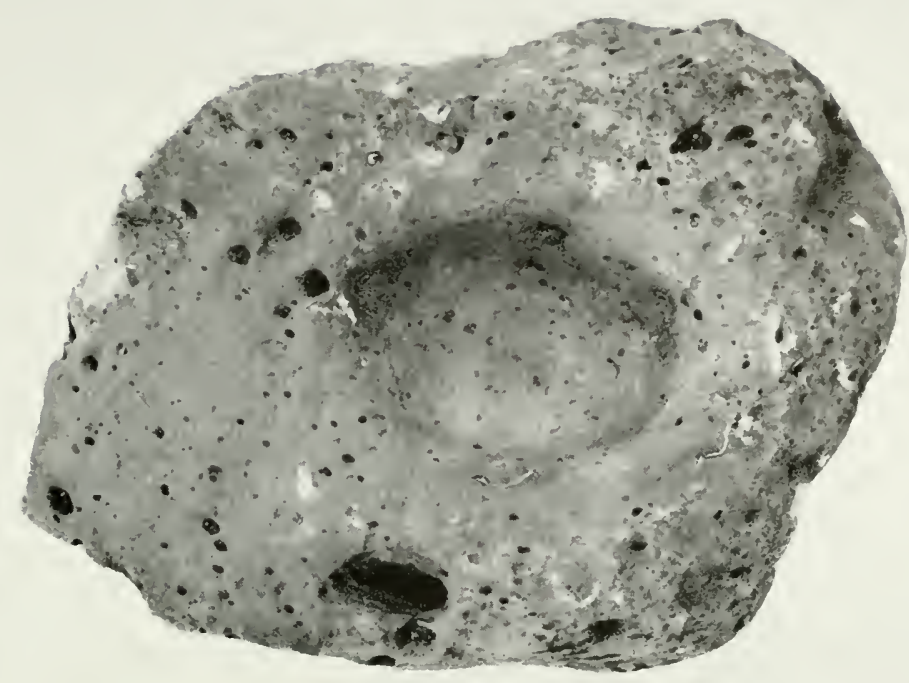

FIG. 55. Mortar from Thode Site. Length, $28.0 \mathrm{~cm}$.

from 4.1 to $13.5 \mathrm{~cm}$. The cups are from 5.0 to $10.7 \mathrm{~cm}$. in diameter and from 0.8 to $7.0 \mathrm{~cm}$. deep. They were recovered from the Goesling and Thode Sites and from Rim Valley and Hooper Ranch Pueblos.

These appear to be similar to mortars and stone bowls from Point of Pines (Wendorf, 1950, pp. 57-58; Breternitz, 1959, pp. 41-42) and from the Reserve area (Rinaldo, 1959, p. 241; Martin and Rinaldo, 1950b, p. 470). Only one specimen was neatly worked on the exterior; the others were rough. However, specimens from the dwelling rooms of the Hooper Ranch Pueblo were more specialized in nature (Martin, Rinaldo and Longacre, 1961, p. 71).

\section{Рот Covers}

(Figure 56, lower)

Two stone discs with flat broad surfaces and rough-hewn edges were recovered, one from the Great Kiva at Hooper Ranch Pueblo, the other from Pithouse $A$ at the Goesling Site. They are 16.2 and $9.8 \mathrm{~cm}$. in 
dianeter respectively, and 2.3 and $1.1 \mathrm{~cm}$. thick. They are similar to objects found in place as jar covers. This type of artifact rare in the Mogolton area - is more frequent on sites to the north such as Tseh Tso (Brand, Hawley and Hibben, 1937, p. 95) and might possibly represent the diffusion of this trait from the north.

\section{HAMmerstones \\ (Figure 53, $c, d$ )}

Tools of this type are fairly common in most Southwestern sites. We collected 29 of them, many from the later sites (Rim Valley Pueblo, Hooper Ranch Pueblo). They are generally angular stones, although some are reduced through use to a round shape. Those we collected were from 5.9 to $11.5 \mathrm{~cm}$. in diameter (or greatest dimension). Marks of use and association with other artifacts indicate that they were used for several purposes - for roughening the surfaces of milling stones (pecking), for pounding, and for percussion flaking.

\section{Рот Rests}

These crude stone objects, which occurred only at the Rim Valley Pueblo, were simple, unshaped oblong blocks of stone set in the firepits to support the cooking pots and hold them against a corner of the firepit. This type of object has been recovered from the dwelling rooms at Hooper Ranch Pueblo, Table Rock Pueblo (Martin and Rinaldo, 1960a, p. 256), and Foote Canyon Pueblo (Rinaldo, 1959, pp. 250-252).

\section{TOOLS USED IN POTTERY-MAKING}

Many of the milling tools such as manos, metates, mortars and pestles, which were used for crushing grain and seeds, were also used for grinding pigments for paint. We found manos, metates and mortars with pigment on their grinding surfaces in the rooms at Rim Valley Pueblo and at Hooper Ranch Pueblo. They were also probably used for pulverizing clay. These tools have been discussed above. Gourd scrapers used for scraping the surfaces of unfired vessels to smooth them were not recovered. We found several polishing stones (fig. 57). These are generally oval in outline and have one flat facet derived from long use. These were found at Rim Valley Pueblo and the Hooper Ranch Pueblo. They range in length from 3.1 to $6.7 \mathrm{~cm}$., in width from 1.9 to $5.1 \mathrm{~cm}$., and in thickness from 1.3 to $4.8 \mathrm{~cm}$. They are all made of dense stone. 

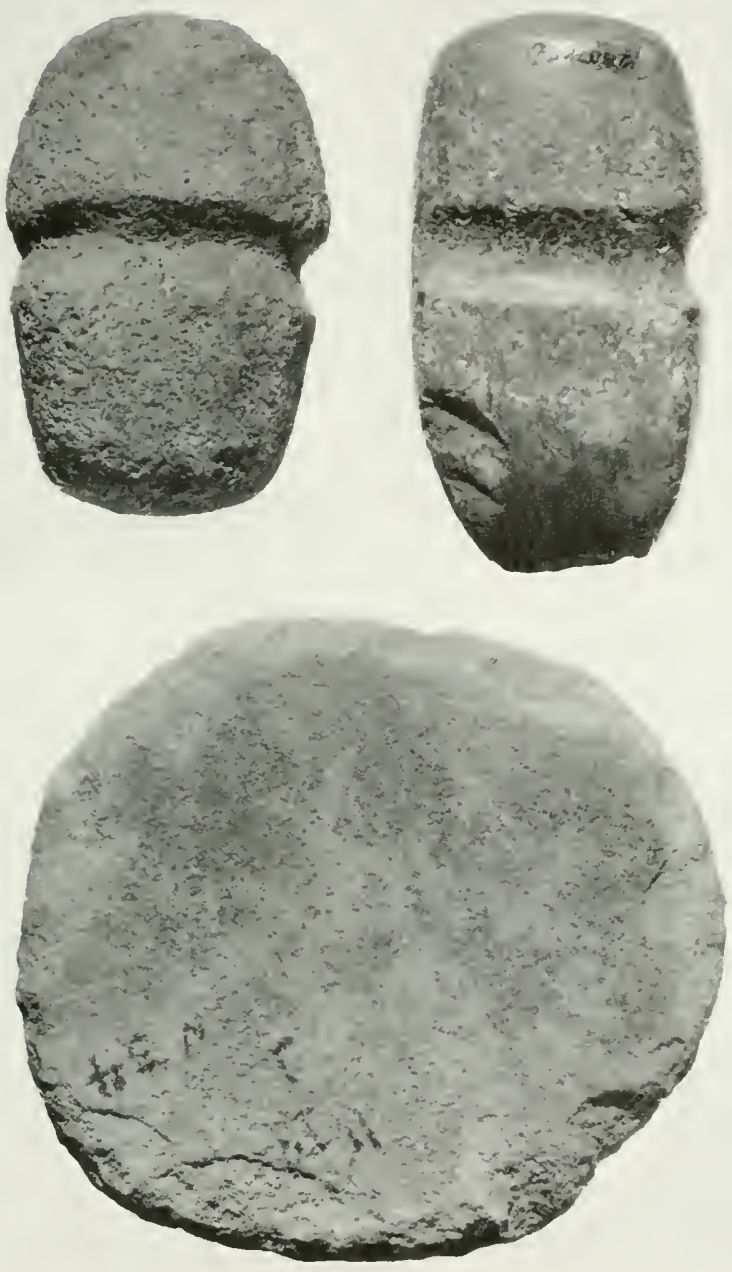

Fig. 56. Maul, axe, and pot cover from Rim Valley Pueblo and Hooper Ranch Pueblo. Diameter of lower spccimen, $16.2 \mathrm{~cm}$. 

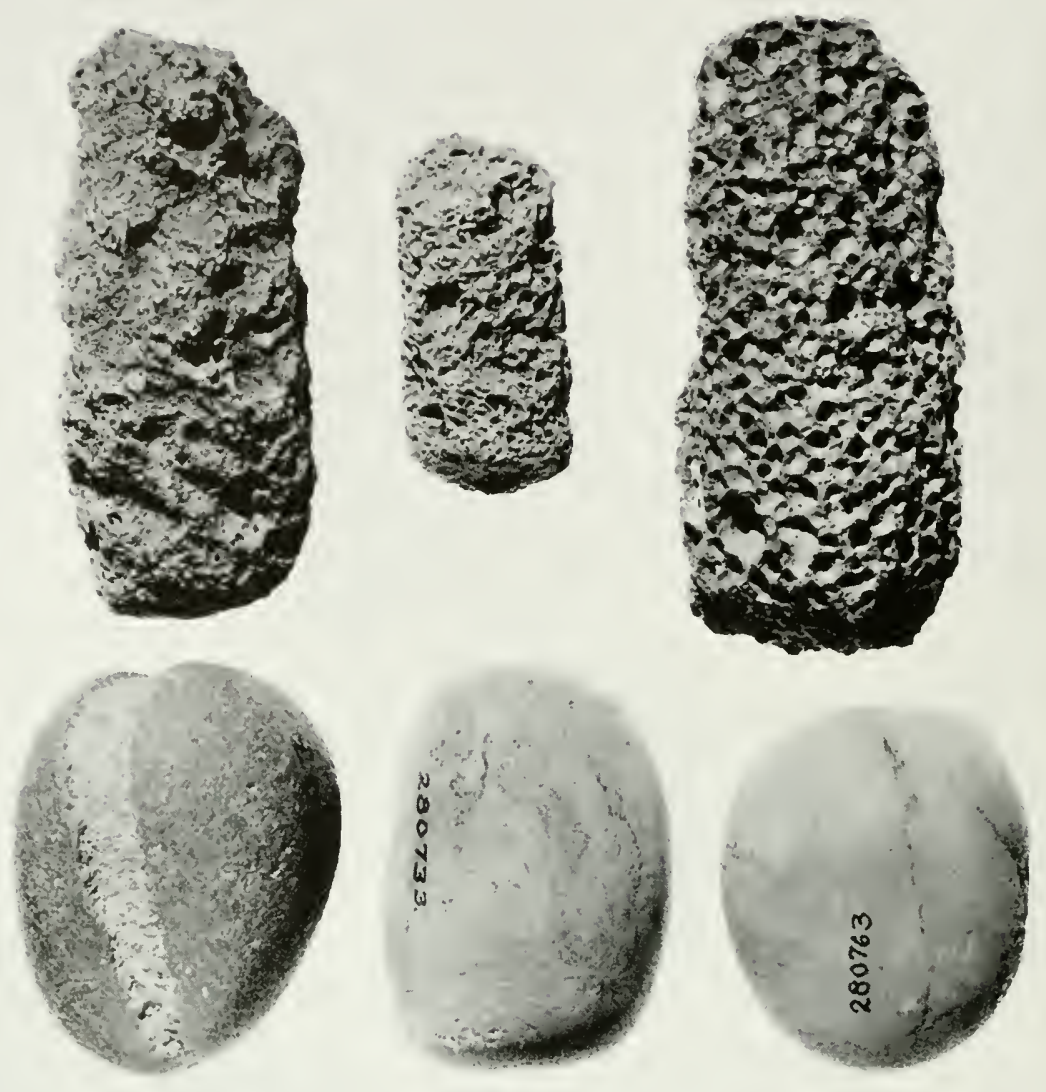

FIG. 57. Medicine cylinders and pot polishing stones from Hooper Ranch Pueblo, Rim Valley Pucblo, and Goesling Site. Length of lower right specimen, $4.1 \mathrm{~cm}$.

\section{TOOLS USED IN THE CONSTRUCTION OF HOUSES}

The few tools described below have traditionally been classified under the category concerned with building. This assignment has been made primarily on the basis of ethnographic information. The provenience of these tools in an archacological context has furnished remarkably little eviclence as to their use. 
One axe and one maul were recovered from Rim Valley Pueblo. Choppers were recovered from three of the eight sites investigated. Axegrinding slabs were found in Rim Valley Pueblo and in the dwelling rooms at Hooper Ranch Pueblo. Axes and mauls were recovered from the dwelling rooms at this pueblo during the 1959 field season.

\section{AxE}

(Figure 56. upper right)

This tool may have been used for cutting the roof members - beams, poles, splints and brush. This axe is of the three quarters grooved type, with the groove located near the middle. The sides and the poll are almost flat. The bit and other broad surfaces are polished. This specimen is $13.2 \mathrm{~cm}$. long. $7.0 \mathrm{~cm}$. wide and $4.9 \mathrm{~cm}$. thick. It is of a form which Rolserts (1932. p. 141) has classified as tabular. The poll is longer than on the majority of three quarters grooved axes, the bit is only medium long.

The three quarters groove tends to classify this axe as a "southern" type. The proportions of the poll and bit are generally similar to those of ases from the Canyon Creek Ruin (Haury, 1934, pl. 71) and from Foote Canyon Pueblo (Rinaldo. 1959, fig. 105).

\section{MAUL}

(Figure 56. upper left)

The maul or grooved hammer was found in the fill of a dwelling room at Rim Valley Pueblo. It is full grooved and has flat faces so that it has been classified as belonging in the tabular type. This implement is made of a heary basalt stone and is $12.0 \mathrm{~cm}$. long, $7.5 \mathrm{~cm}$. wide and $5.8 \mathrm{~cm}$. thick.

Full-grooved mauls appear early in the Mogollon culture but they appear to decrease in popularity. They are not as abundant on the later sites as on the earlier ones. The full-grooved type had a more widespread use than the three quarters grooved type.

\section{Axe-Grinding Slabs}

(Figure 58)

In one surface these thick slabs or blocks have a broad shallow groove of the shape and size that would be produced by grinding a stone axe. The width of the groove corresponds roughly to the size of the blades of the axes recovered from ruins in this area. 
Similar grooves, although usually in bedrock, have been reported from other sites in the Mogollon area (Hough, 1914, p. 4; Martin, et al., 1952, p. 38: Rinaldo, 1959. p. 244). They were recovered only from Hooper Ranch Pueblo and Rim Valley Pueblo during the 1960 season.

\section{Choppers}

(Figure 59)

Rough choppers were probably used for many other purposes besides cutting roofing timber for houses, but apparently axes replaced them during the later periods and so they are included here with axes. They are usually of a convenient size to grasp in the hand. One edge is steeply chipped by percussion to form a sharp cutting edge, and usually a portion of the natural smooth surface of the stone on the opposite margin is left intact to provide a comfortable place to grasp the implement. They range in length from 7.2 to $9.8 \mathrm{~cm}$., in width from 6.1 to $9.1 \mathrm{~cm}$., and in thickness from 2.9 to $6.2 \mathrm{~cm}$.

Choppers occurred at most of the sites that we excavated. The biface type (with cutting edge chipped from both surfaces) was found only at the two latest sites - Rim V'alley Pueblo and Hooper Ranch Puebloand in general the uniface type of choppers appears to be more common in the upper Little Colorado drainage. Our sample may be inadequate because this seems to contrast somewhat with the distribution of similar implements farther south where biface choppers are found throughout the sequence (Haury, 1950, Table 19; Martin, et al., 1952, fig. 71).

\section{TOOLS AND WEAPONS USED IN HUNTING AND WARFARE}

Although we recognize the fact that many of these tools were used in several activities, we follow traditional practice in assigning a category to them, as we did with the construction tools. Projectile points, blades and even some small oval biface scrapers tend to grade from one category to the other, so for this reason also we cannot be specific about uses.

\section{Projegtile Points}

The small triangular lateral notched projectile points (fig. $60, i-l$ ) appear to occur consistently late in this sequence as they do in neighboring areas. The specimens in the collection from the Hooper Ranch Pueblo and the Thode Site are not uniform in certain details such as the placement of the notches, but as a group they are easily distinguished from the larger diagonal notched points, for example. 
A similarity may be seen between these small, triangular. lateral notched points and some from Point of Pines (Wendorf, 1950, fig. 32, $b-f$; Breternitz, 1959, pp. 47, 66, fig. 38, $k, r$ ) and from the Reserve arca (Martin, Rinaldo, and Bluhm, 1954, p. 125, fig. 64, $k-u$; Martin, Rinaldo, and Barter, 1957, fig. 42, $f-i$; Nesbitt, 1938, pl. 47, B). Furthermore, although the resemblance is not as close as it is to points from the Mogollon area, some points from Pueblo III sites in the Anasazi area tend to be similar in style (Judd, 1954, p. 255, pl. 73, B, a-e; Roberts, 1932, p. 146, pl. 59, $a-\ell)$.

On the other hand, the projectile points from the earlier sites (fig. 60, $a-d, h)$ tend to be larger and have barbs which the later points lack. Although some of these points are lateral notched, more of them tend to be diagonal notched.

Only five notched or shouldered projectile points (fig. 60, $a-d$ ) were recovered from Tumbleweed Canyon Site, each one of them quite different from the others. All are of medium size ( 3 or $4 \mathrm{~cm}$. long). Two are diagonal notched, one is comer notched with a broad straight stem and two have expanding bases with small shoulder-like projections. These correspond roughly to points from the Pine Lawn Phase (Martin, 1943, fig. 72, B, fig. 73, E) and from the Circle Prairie Phase (Wheat, 1954, fig. 54, $f, h-k)$. This scarcity of notched points contrasts markedly with the number of blades (fig. 61) and bases without notches (42). Many of these are thin and must have been used as projectile points or knives. Others are thick and steeply chipped at the edges and may have been used as scrapers.

\section{Arrow-Shaft ToOls}

The arrow-shaft straightener (fig. 62,c) is the only one of its kind recovered. It may be only partly finished inasmuch as the groove is rough, not polished like the grooves on most tools of this kind. It is made from a thin rectangular basalt pebble and the groove runs lengthwise down the middle. It came from the Great Kiva at the Hooper Ranch Pucblo and is $6.5 \mathrm{~cm}$. long, $5.6 \mathrm{~cm}$. wide, and $1.6 \mathrm{~cm}$. thick.

An arrow-shaft smoother (grooved abrader; fig. 62, a) came from the same structure. This has a longitudinal groove of the same width from end to end.

Both of these are simple tools, although broken examples of more claborate types came from the same site (Martin, Rinaldo, and Longacre, 1961, p. 102). Arrow-shaft tools are usually scarce on sites earlier than the Tularosa Phase or Pueblo III. They occur predominantly on late pueblo 

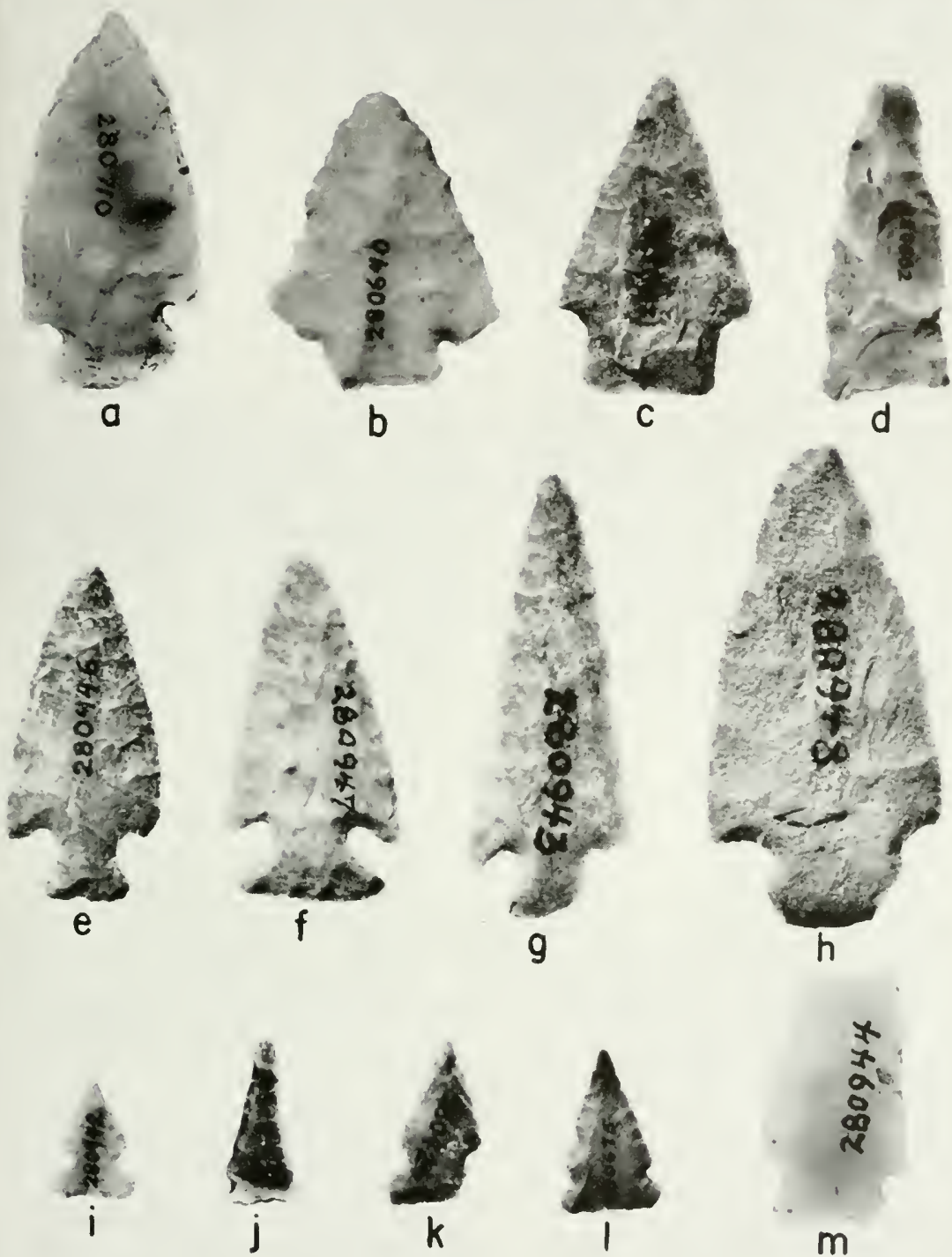

Fig. 60. Projectile points, miscellancous types. Length of $m, 3.0 \mathrm{~cm}$. 

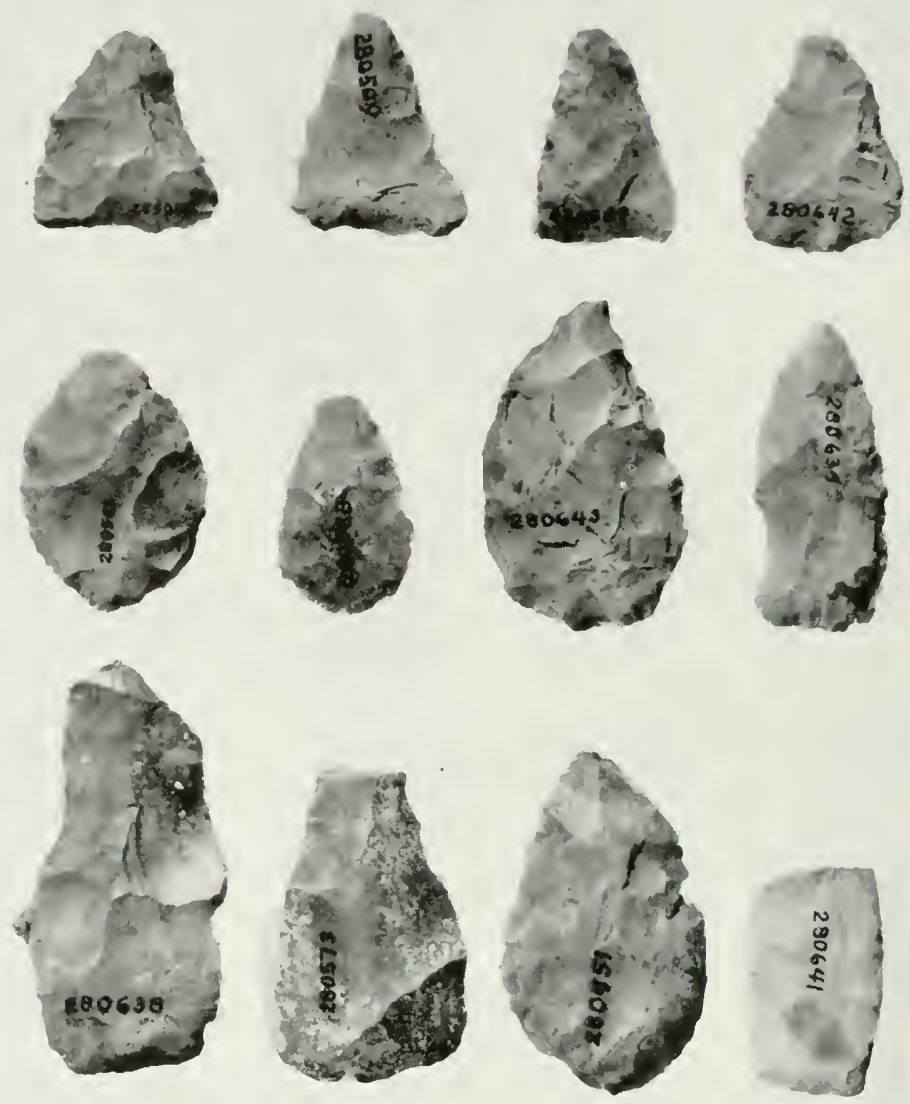

Fig. 61. Blades from Tumbleweed Canyon Site and Hooper Ranch Pueblo.

sites and in a general Upper Gila, Little Colorado, Rio Grande distribution. They are rare even on a late horizon in the neighboring areas to the north or south.

These tools need not necessarily have been used for shaping arrow shafts or foreshafts; they could have been used for smoothing prayer sticks or spindles. 


\section{HOUSEHOLD UTILITY TOOLS}

The tools included in this category are those that probably were used for several purposes. These include cutting edges such as flake knives, saws, and scrapers, and perforators such as drills and punches.

\section{Flake Kivives \\ (Figure 63, c.j)}

Approximately 270 utilized flakes were recovered from the eight sites. These range from thin flakes with some almost microscopic chipping along one edge to somewhat thicker flakes with well-defined secondary

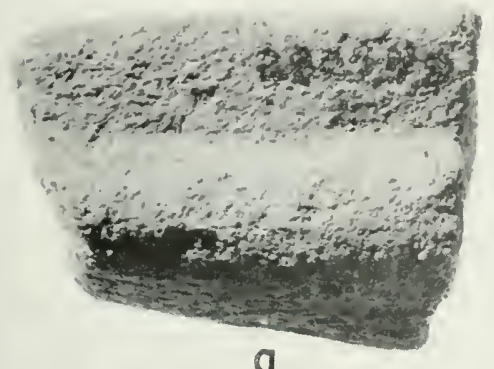

a

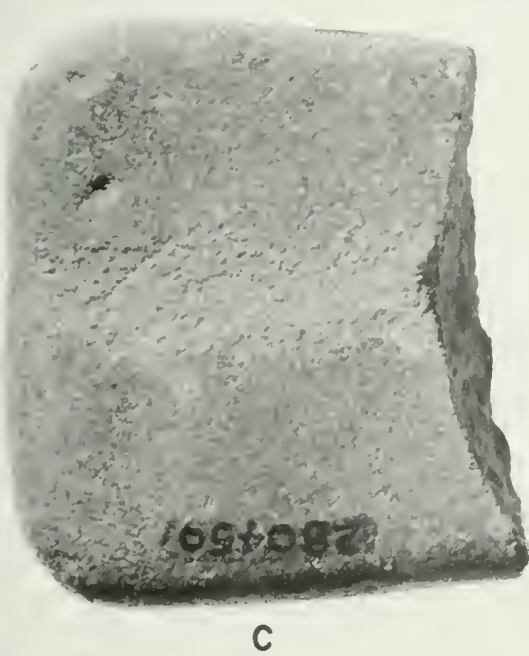

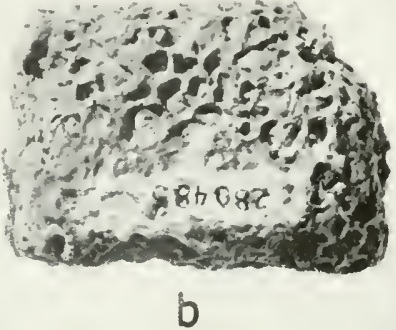

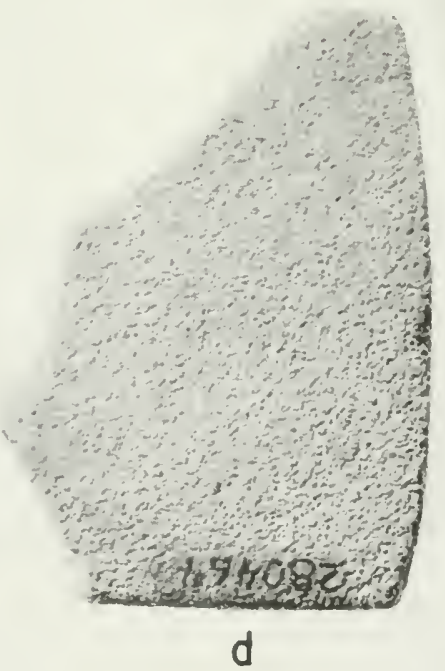

FIG. 62. Abrading stones $(a, b)$, arrow-shaft tool $(c)$, and smooth saw (d) from Hooper Ranch Pucblo. Length of $d, 6.4 \mathrm{~cm}$. 
chipping on one broad surface and along more than one edge. Nlthough these are far from uniform in shape of outline, they tend to be oblong. Most of them are made from chert or chalcedony flakes, but there are some fine-grained basalt, fine-grained quartzite and felsite flakes.

They have diminishing distribution from early to late. Almost half were recovered from Tumbleweed Canyon Site (122). The Chilcott Site 1 yielded over a sixth. the Thode Site and Rin Valley Pueblo about a sisth each, and the Hooper Ranch Pueblo Great Kiva about a twelfth; the remainder are divided between the three smaller excavations, which do not seem comparable. This decline in popularity seems to parallel that which appeared in the areas to the south wherever comparable information is available (Martin, et al., 1952, p. 489).

Thin cutting edges were probably used something like our pen knives for whittling wood (arrow and dart foreshafts, prayer sticks, spindles), cutting other soft materials (leather, string, fiber), or stinning animals.

\section{SCRAPERS \\ (Figure 63. $a, b, d-i$ )}

Approximately 116 small scrapers were recovered. Although they were found at all of the sites, the majority of them (64) come from the Tumbleweed Canyon Site. The next largest group (29) is from Hooper Ranch Pueblo. These are only a little more specialized in form than the flake knives. They are small $(2.0$ to $7.0 \mathrm{~cm}$. long: 1.6 to $5.7 \mathrm{~cm}$. wide), rough, thick $(0.4$ to $4.0 \mathrm{~cm}$.) flakes with some secondary chipping at a steep angle along one or more edges. They are generally plano-convex in cross section and the convex surface was shaped by percussion chipping. The available information, though inadequate for a truly accurate estimate, appears to indicate a decrease in these tools similar to that of the flake knives.

The large scrapers might be classified as scraper-planes. They are generally plano-convex in cross section and have steep secondary chipping along one edge. There were not enough of these found to consider the distribution significant in indicating an increase or decrease in popularity. They came from both early and late sites: Tumbleweed Canyon Site (3), Chilcott Site 1 (4), Rim Valley Pueblo (4), and Hooper Ranch Pueblo, Great Kiva (1).

\section{SAWS}

\section{(Figure 64, i, j)}

These implements are thin flakes with one or more margins chipped into a series of deep notches to form a serrate cutting edge. Only two of 

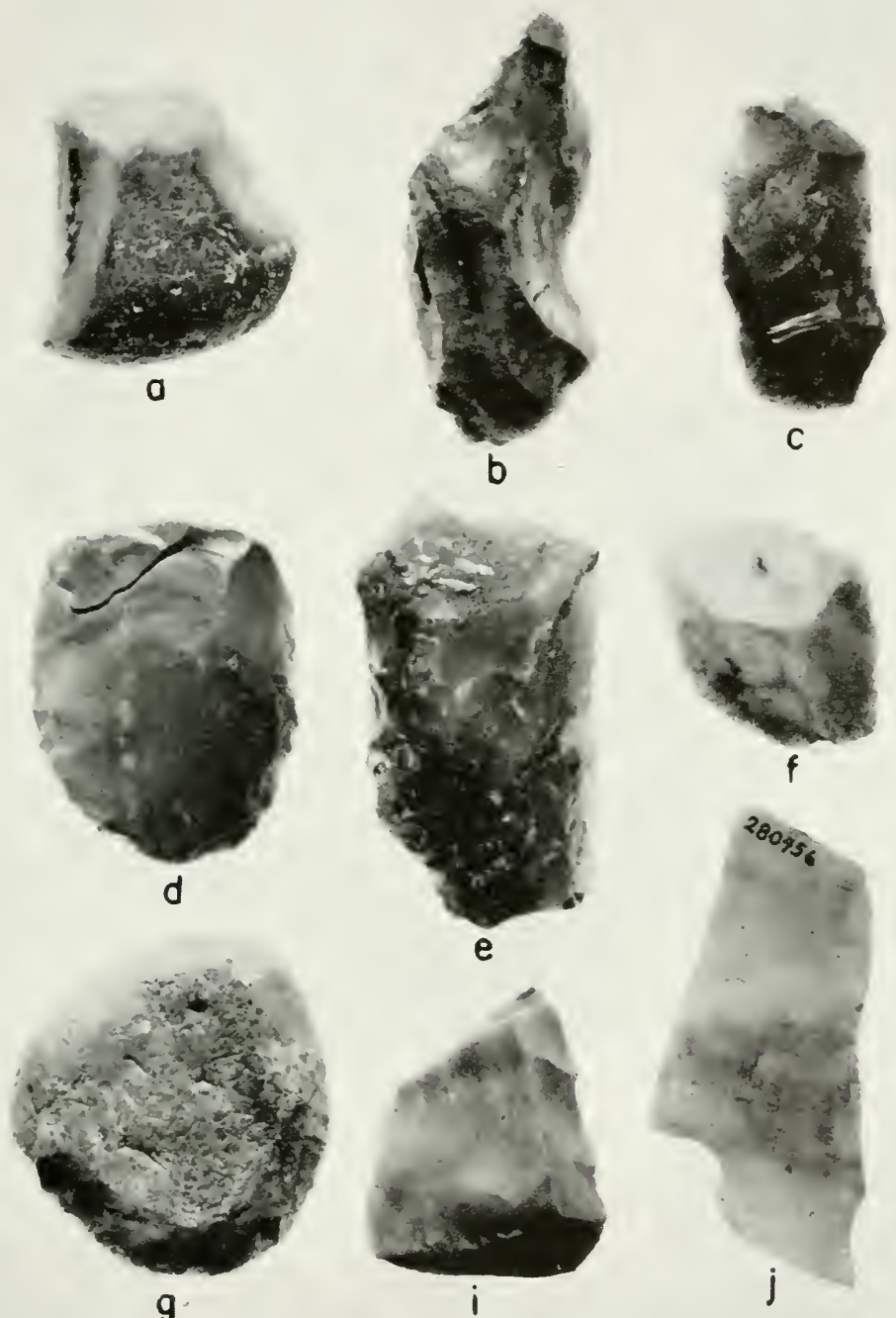

FIG. 63. Flake knives $(c, j)$ and small scrapers $(a, b, d-i)$ from Tumbleweed Canvion Site, Chilcott Site 1, Goesling Site, and Hooper Ranch Pueblo. Length of $j, 5.8 \mathrm{~cm}$. 


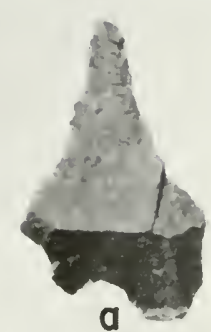

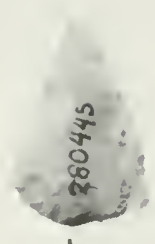

b

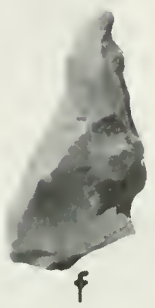

C
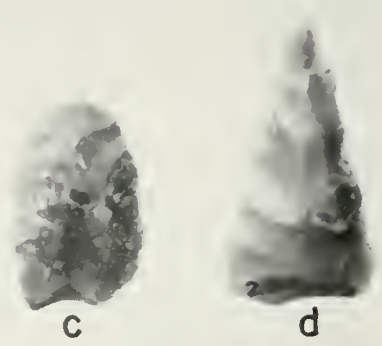

e

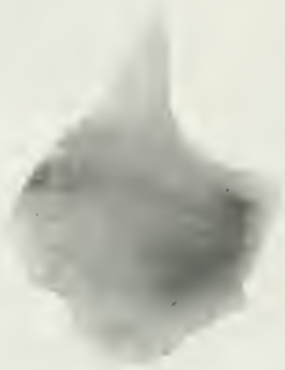

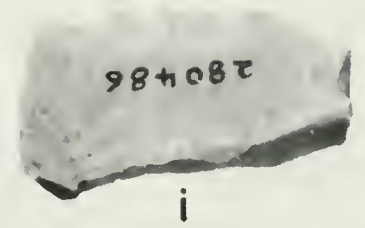
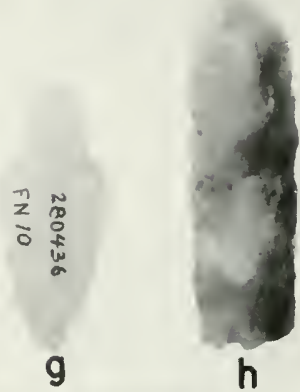

9

h

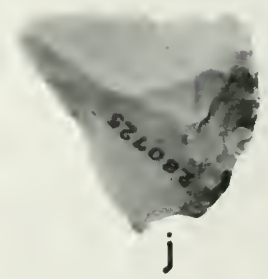

FIG. 64. Drills, punches, saws, and blades from Hooper Ranch Pueblo, Goesling Site, Chilcott Site 1, and Rim V'alley Pueblo. Length of $j, 3 \mathrm{~cm}$.

these saws were recovered, one from Hooper Ranch Pueblo, Great Kiva, and one from Rim Valley Pueblo. This late distribution is in accord with their occurrence in the Reserve area, where they are found in the San Francisco Phase at the earliest; eren at Forestdale (Haury, 1940, p. 107) they do not occur earlier than the Forestdale Phase. There are some surface indications that they may occur earlier north of Concho (Bretcrnitz, 1957, p. 78).

One square-cornered fragment of a smooth saw (fig. 62, d) was found in the Great Kiva at Hooper Ranch Pueblo. Only enough of the 
cutting edge remains to enable one to classify it. These tools also occur primarily in late sites (Rinaldo, 1959, p. 252).

\section{DRILLS \\ (Figure 64, $a, e^{-h}$ )}

A half-dozen drills were recovered from the sites, but only one or two came from each site. One is the plain-shafted type and the others are the flanged type. The drills in this latter group have slender points which taper from broad bases. They average $3.7 \mathrm{~cm}$. in length.

Because drills are one of the less common chipped stone artifacts in the Southwest - although they are not rare-our present generalizations on their distribution will be subject to revision. However, they do appear to be more frequent in the very early levels and sites and relatively less frequent in the later ones.

\section{WEAVING TOOLS}

Evidence seems to indicate that bone awls and bodkins were used in weaving baskets and in separating the warp and weft elements on the loom. Molded spindle whorls were, of course, used as a form of fly wheel on spindle shafts in the process of spinning yarn (Kent, 1957, pp. 472-473). Some of the more symmetrical worked sherds with holes bored through their centers may also have served as whorls.

\section{Bone Awls \\ (Figure 65)}

These implements were recovered from the Goesling Site, the Thode Site, Rim Valley Pueblo and Hooper Ranch Pueblo. They fall into four types: (1) the ulna type (fig. $65, k$ ); (2) those made from split long bones with the condyle unaltered except by the original splitting (fig. $65, a, d, f-i) ;(3)$ the same type as No. 2 except that the condyle is partly worked down (fig. 65, c); (4) awls made from bone splinters (fig. 65, b, $, l, l$ ).

Only the ulna type appears to have a significant distribution. The only specimen of this type found during the 1960 season comes from the latest site. Although they have been recovered from earlier sites in prior seasons, they are more frequent from the later sites (Martin and Rinaldo, 1960b, p. 229). This was also true in the Reserve area (Rinaldo. 1959, p. 264), and it appears to be the situation at Point of Pines (Wendorf, 1950, pp. 77-79; Wheat, 1954, pp. 159-160; Breternitz, 1959, p. 51). 
The bodkin tip (fig. $65, n$ ) is a broad, flat, blunt point decorated with an incised zigzag line. It was found in Rim Valley Pueblo. Another scrap of bone (fig. $65, \mathrm{~m}$ ) is decorated with a "negative lightning" design formed by a pattern of incised lines and dots. This was recosered from Hooper Ranch Pueblo. Art work of a generally similar nature was seen in a bone effigy pendant from the dwelling rooms at Hooper Ranch Pucblo (Martin. Rinaldo and Longacre, 1961, p. 106).

Most of the awls have tips that are round in cross section. Only two (in addition to the bodkin) hase tips that are laterally flat. These may have been used for a different function than the round pointed awls, such as plaiting (Di Peso, 1956, pp. 414-415).

\section{SPINDLE Whioris}

(Figure 66)

Two modeled clay spindle whorls (fig. $66, c, d$ ) were recovered from Chilcott Site 1. These are a gray color and resemble a pulley or a spool in shape. They are about $3 \mathrm{~cm}$. high and slightly less than that in diameter. Similar whorls were found at the Los Muertos Site (Haury, 1945, fig. 71, i, j, p. 117); Babocomari Village (Di Peso, 1951, p. 107, pl. 44, D); Tuzigoot Ruin (Caywood and Spicer, 1935, p. 66, pl. 15. D); and Snaketown (Gladwin, et al., 1937, p. 245, pl. 212, m).

Sites in the Papagueria of southern Arizona have produced more modeled clay spindle whorls than sites farther north (Haury, 1950, p. 360) or in other sections of Arizona, and this culture element is believed to be of Mexican derivation (Di Peso, 1956, pp. 396-397). Although they have been found as far north as Flagstaff (McGregor, 1941, pp. 74-76) they are more common farther south. With the exception of a few specimens from Tularosa Cave in San Francisco levels (Martin, et al., 1952, p. 196) they generally are late in occurrence.

Ten disc-shaped worked sherds (fig. $66, e, f$ ) perforated through the center were found. Seven of these came from the Goesling Site, one from the Thode Site and two from the Hooper Ranch Pueblo Great Kiva. These are common in pottery-bearing sites throughout the Southwest.

\section{ORNAMENTS}

Stone and shell pendants and beads as well as fragments of shell bracelets and a ring are classified as ornaments. A few small, unperforated objects of a shape similar to that of the pendants but a little larger possibly should be included as unfinished pendants. One of the pendants is 

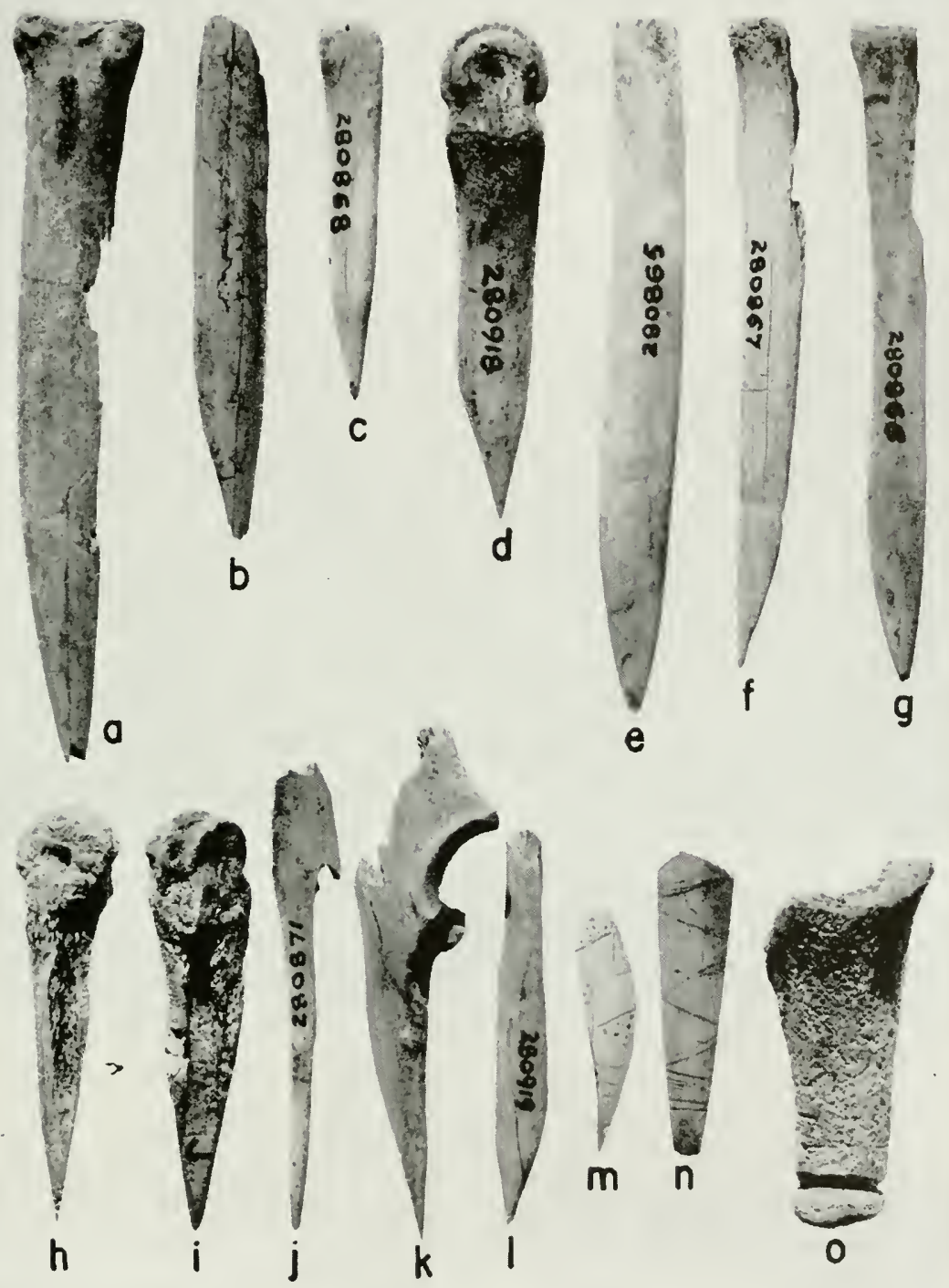

FIG. 65. Bone awls, incised bone fragment, bodkin tip and ring material from Goesling Site, Rim Valley Pueblo, Hooper Ranch Pueblo, and Thode Site. Length of $o, 6.7 \mathrm{~cm}$. 

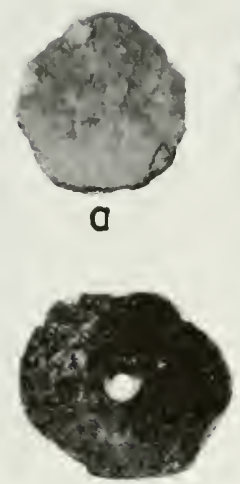

e

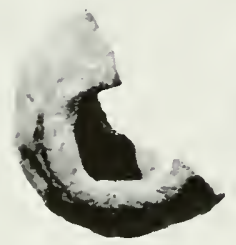

g
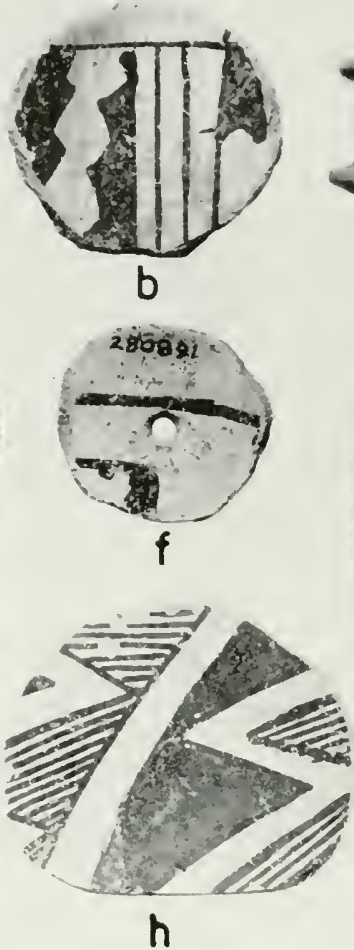
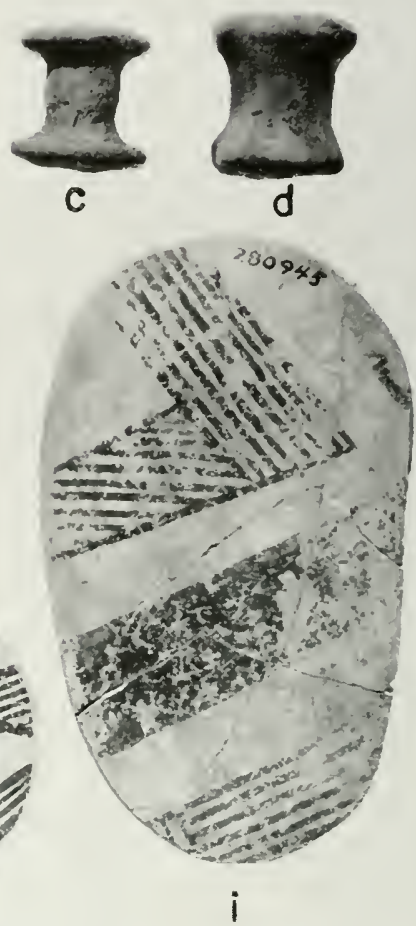

Frg. 66. Spindle whorls, worked sherds and miniature jar from Chilcott Site 1, Goesling Site, and Hooper Ranch Pueblo. Length of $i, 11.9 \mathrm{~cm}$.

grooved near one end; the others are perforated near one end. The beads are all centrally perforated.

None of the ornaments is elaborately decorated or shaped. Incised designs or even conventionalized zoomorphic shapes are lacking and only types simple in nature were recovered. On these sites ornaments were relatively rare or absent. In general they appear to be more plentiful and elaborate in sites in the southern part of Arizona than they are in the north. 


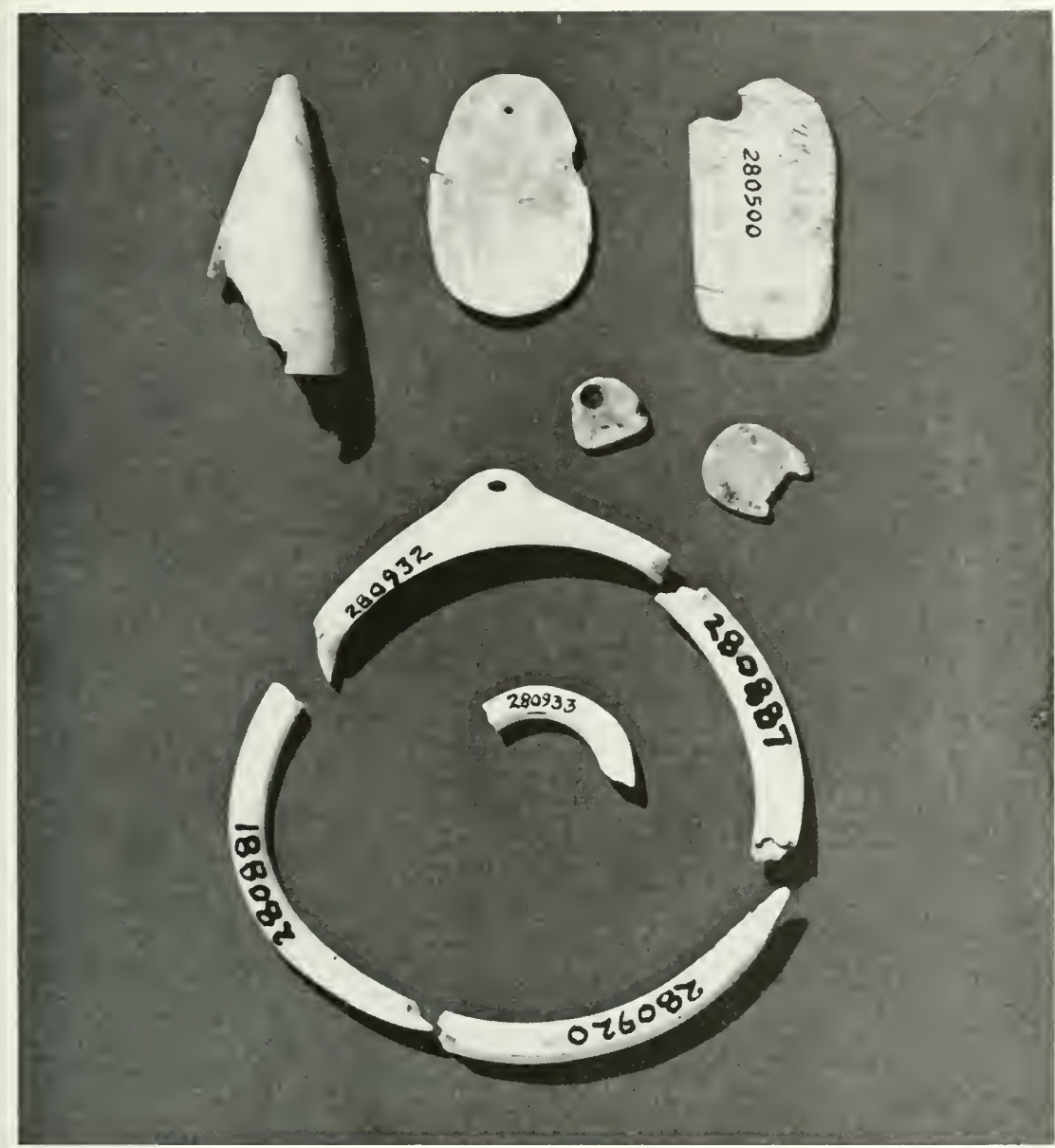

FIG. 67. Pendants, bracelet fragments, and ring fragment from Goesling Site and Hooper Ranch Pueblo.

\section{Pendants}

(Figure 67, upper right)

A few tabular pendants were obtained from the digging. Two came from the Hooper Ranch Pueblo (one of turquoise, one of bone) and two from the Goesling Site (another of turquoise and one of clam shell). A fifth pendant, of white limestone, is grooved near one end for suspension. This came from Rim Valley Pueblo. The bone, shell and white stone pendants are oblong in shape, and are longer than wide. The turquoise pendants are almost as wide as they are long. One is roughly triangular in shape, the other rectangular with rounded corners. 


\section{BeAds}

The beads are of two types: small, centrally perforated dises and what is sometimes termed "truncate olivella" (Morris, 1919, p. 93). All of these came from the Hooper Ranch Pueblo. Both types have been recovered from both early and late sites of the Mogollon and Anasazi cultures.

\section{Bracelets}

(Figure 67, lower)

These specimens are all fragments of the thin type of shell bracelets, and all came from Pithouse $\mathrm{A}$ at the Goesling Site. This type appears to have an over-all distribution, occurring throughout the Southwest in both early and late sites and levels. The thick type, which occurred in a dwelling room at Hooper Ranch Pueblo (Martin, Rinaldo and Longacre, 1960 , p. 45) but not in the excavations made during the 1960 season, appears to be late in its distribution (Gladwin, et al., 1937, p. 142; Nesbitt, 1938, p. 108).

\section{Rings \\ (Figure 67, lower center)}

One fragmentary shell ring was found in Pithouse A, at the Goesling Site, and is the only specimen of this type recovered.

Shell rings are not as common as bone rings in sites north of the Mogollon Rim. Shell rings have been reported from Pueblo Bonito (Pepper, 1920, p. 371), Snaketown (Gladwin, et al., 1937, pp. 144-145), the San Simon Village (Sayles, 1945, pl. 52), Quiburi (Di Peso, 1953, p. 179), San Cayetano (Di Peso, 1956, p. 95), and Arizona W $10: 51$ (Wendorf, 1950, p. 89).

\section{Bone Ring Material \\ (Figure 65, o)}

This specimen is mentioned here as further evidence of the use of bone rings by the people of Hooper Ranch Pueblo. It is a hollow section of long bone including a portion of the condyle. A deep groove encircles the end opposite the condyle. The specimen is $6.7 \mathrm{~cm}$. long and $2.4 \mathrm{~cm}$. in diameter.

A few rings and several specimens of long bones prepared as ring material were recovered from the dwelling rooms (Martin, Rinaldo and Longacre, 1960 , p. 50). These rings have been recovered mostly from 
late ruins south and west of Zuni such as Arizona $\mathbb{W}: 10: 51$ (Wendorf, 1950, p. 81), Kinishba (Baldwin, 1939b, p. 321), Canyon Creek (Haury, 1934, p. 126), Hawikuh (Hodge, 1920, p. 145), and Pinedale (Haury and Hargrave, 1931, fig. 16).

\section{TINKLER}

(Figure 67, upper left)

A single fragmentary specimen of a conical tinkler was recovered from the Great Kiva at Hooper Ranch Pueblo. Near the smaller end there is a notch through which a string could be passed.

Similar conus tinklers come primarily from late horizons after A.D. 1100 and from as far east as Pecos (Kidder, 1932, p. 190), as far west as the Elden Pueblo near Flagstaff (McGregor, 1941, p. 281), north in the Aztec Ruin (Morris, 1919. p. 94), and south in the Hohokam sites (Haury, 1945. p. 149: Gladwin, et al., 1937, p. 145), and at San Cayetano (Di Peso, 1956. pp. 91-92).

In the upper Little Colorado drainage, they also occurred at Table Rock Pueblo (Martin and Rinaldo, 1960b, p. 225) in the larger of two kivas.

\section{Cut Shell}

One very small piece of cut shell with a notched serrate edge was found at Chilcott Site 1. Cut shell pendants or mosaic pieces with nacreous surfaces and scalloped or notehed edges were recovered at Los Muertos (Haury, 1945, p. 150) and Snaketown (Gladwin, et al., 1937, p. 142, pl. 119). Cut shell specimens seem to have a distribution primarily in the Hohokam area.

\section{CEREMONIAL OBJECTS}

Three groups of objects are included in this category: cylinder stones, the sacred stone image, and some of the worked sherds.

\section{Cýlinder Stones \\ (Figure 57, upper)}

These rough approximately cylindrical objects are made of vesicular basalt. Five of them were found, three in the Great Kiva at Hooper Ranch Pueblo, one at Rim Valley Pueblo, and one at the Thode Site, the latter two in rooms. 
These objects have a distribution that extends from the SU Site (Martin and Rinaldo, 1940, p. 62, frg. 28) west as far as Tuzigoot (Caywood and Spicer, 1935, p. 86). They are common in the llagstaff area (Bartlett, 1934, p. 26; McGregor, 1941, p. 178; Snith, W., 1952a, pp. 127129), and occur in the Jeddito area (Woodbury, 1954, pp. 181-182). They appear to be more common on the earlier horizons than on the later.

\section{SACred Stone IMAge}

(Figure 42)

This object is treated at length in Chapter II. Although stone images of animals, corn mounds, crosion remnants, petrified wood and fossils have been found frequently in prehistoric sites, anthropomorphic images apparently were comparatively rare before the Spanish conquest (Fewkes, 1924. pp. 377-397: Martin, et al., 1956, pp. 84, 94). They have been reported from Pecos (Kidder, 1932, pp. 86-91; Lambert, 1957, pp. 9396) and Te'ewi (Wendorf, 1953, pl. 35). but these are the only prehistoric specimens our search has rerealed unless a stone ornament from Pueblo Bonito (Judd, 1954, fig. 20, o) is so considered.

\section{Worked Sherds}

(Figure 66, $a, b, e, f, h, i$ )

Most of the 23 disc-shaped worked sherds that were recovered came from the Goesling Site. One came from the Chilcott Site 2 and one from the Hooper Ranch Pueblo.

Worked sherds of other shapes-oval, triangular, fragmentary-were more evenly distributed. Out of 22 specimens in the mixed group, 10 came from the Goesling Site, 8 from Chilcott Site 1, 2 from Rim Valley Pueblo and 2 from Hooper Ranch Pueblo. These are about 5.0 cm. in greatest dimension.

Throughout the Southwest and in other areas to the south and east, worked sherds have been found in most sites where pottery has been recovered.

\section{SUMMARY}

The distribution of the stone, bone and shell artifacts generally corroborates certain trends and developments observed previously in both the excavated and the surveyed sites, but it also adds details to previously incomplete observations. The following artifact types appear to decrease in frequency from early to late: (1) one-hand manos. (2) basin 
type metates, (3) rubbing stones, (4) mauls. (5) drills, (6) barbed projectile points. A few others appear to increase in frequency from early to late: (1) long two-hand manos, (2) beveled manos, (3) small triangular projectile points, (4) trough type metates. (5) bone awls made from ulnas. A few traits have only a late distribution: (1) arrow-shaft tools, (2) pot rests, (3) bone rings, (4) shell rings, (4) conus tinkler pendants. Another small group of traits appears to have come into the area as the result of trade, gifts, or some other means. These are the modeled clay spindle whorls and the shell ring, and possibly the three quarters grooved axe, all of which seem to be southern in origin. Stone cylinders and stone disc-shaped pot covers could have been northern elements, although they appear in much greater frequency than the former traits and probably were more completely accepted into the local culture.

On the whole, though, the basic tools of the culture such as the manos, metates, choppers, scrapers, flake knives, and projectile points continued in use throughout the sequence of the upper Little Colorado culture and were subjected only to a slow process of modification. 


\title{
VII. Archaeological Reconnaissance in Eastern Arizona
}

\author{
By William A. Longacre \\ Field Assistant
}

\section{INTRODUCTION}

An archaeological surface survey under the auspices of the National Science Foundation was conducted in east-central Arizona as part of the 1960 Southwest Expedition of Chicago Natural History Museum. This investigation extended the survey begun in the previous year. During the two seasons more than 5,000 miles were covered by truck, and an intensive reconnaissance of approximately 50 square miles was made on foot. As a result, 170 new sites were discovered.

In 1959, work was concentrated in the valley of the Little Colorado River from St. Johns to Springerville and in a large triangular area outlined by Highways 60 and 61 as they merge east of Show Low, the right side of the triangle being Highway 666 between Springerville and St. Johns. In addition, some time was spent in the foothills of the White Mountains south and west of Springerville, and in the region near Snowflake and Mesa Redonda.

In 1960, the survey concentrated upon the Snowflakc-Mesa Redonda region and the White Mountains south of an imaginary line drawn between Show Low and Springerville. Further reconnaissance was carried out in the Little Colorado River Valley between St. Johns and Springerville, and in the area surrounding Show Low.

The region investigated in this survey (fig. 68) occupies a portion of the Colorado Plateau in east-central Arizona. It includes part of the upper clrainage of the Little Colorado River and portions of several of its tributaries. It is roughly outlined by parallels $34^{\circ} 30^{\prime}$ on the north and $34^{\circ} 20^{\prime}$ on the south, with meridians $109^{\circ} 20^{\prime}$ and $110^{\circ}$ the eastern and western boundaries, respectively. The topographical and ecological setting of the survey area has been described elsewhere (Martin, Rinaldo, and Longacre, 1961, pp. 150-153). 


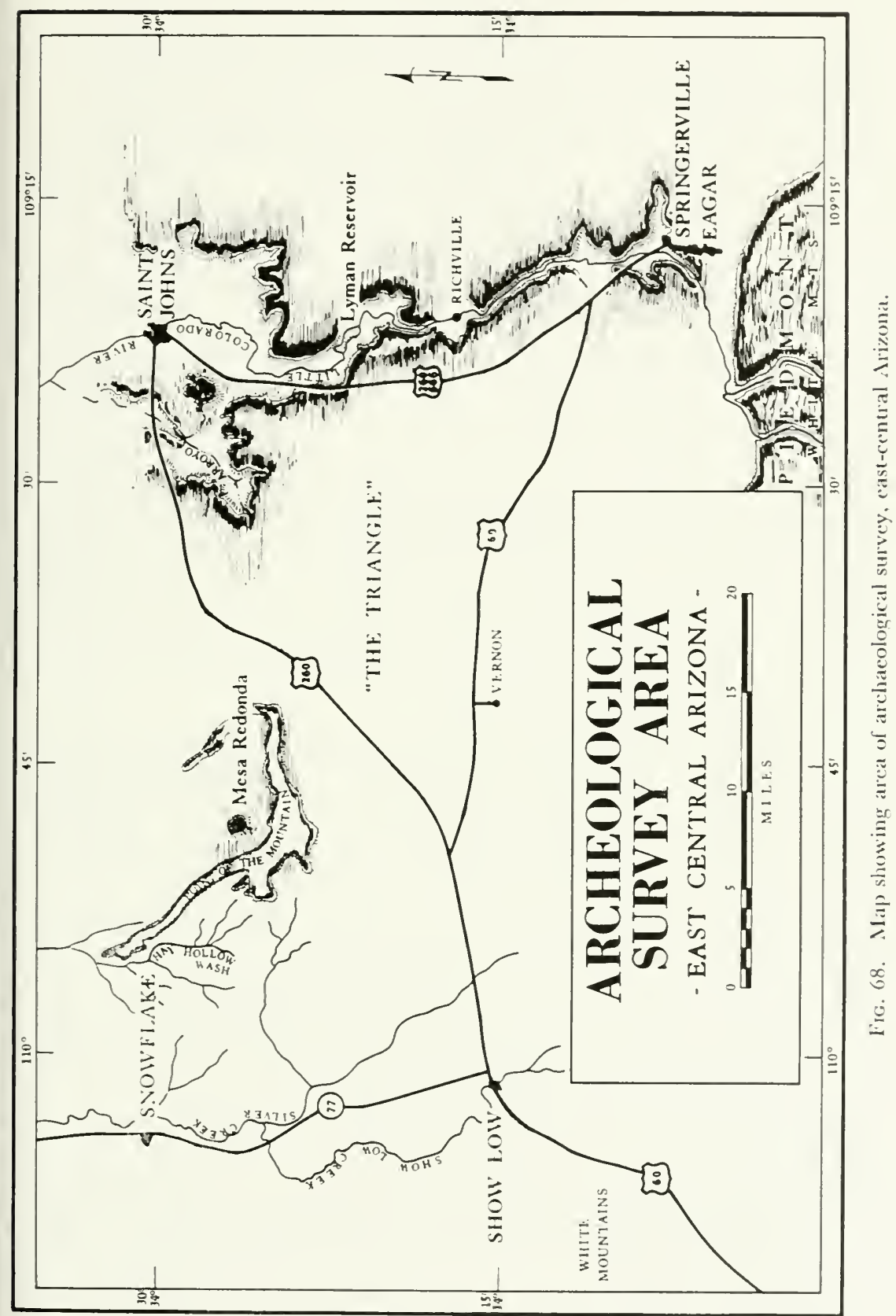




\section{ORGANIZATION}

As in the report prepared on the 1959 survey (op. cit.), the following terminology has been used:

I site is defined as an area used by prehistoric peoples as indicated by cultural detritus. This rather inclusive definition would include areas of occupation ranging from small camp-sites with no evidence of architecture to large above-ground structures of more than 100 rooms.

It was decided to record each site only once, and later each site was broken down into occupational components and labled "a," "b," "c.," etc., on the basis of the pottery analysis. When this was done, the 107 sites recorded during the 1959 season expanded to 154 temporally different components, and the 63 sites found during the 1960 season, to 89 components.

Presentation of the material in a temporal framework presented a problem. The Pecos Classification proved to be unsatisfactory because it was too general. The discrepancy between time and cultural inclusion in it is recognized. The system of phase-designation is now accepted by most archacologists in the Southwest and therefore would be the ideal way to present this report. Information gained from excavation, however, is relatively incomplete in the surveyed area, and I could not justify an attempt to assign phases.

I decided to present the material in the form of rough time periods based upon admittedly arbitrary "groups," with dated pottery types or the absence thereof as the delimiting factors. It is hoped that the presentation of the material in this manner will better facilitate a later incorporation into future archacological studies within the area.

The use of the term "group" here does not connote the exact meaning that Colton gave "Ceramic Group" (Colton, 1946, pp. 18-20). Below is a list of groups, pottery types included, and tentative dates:

Group I.-Pre-pottery: absence of pottery, stemmed projectile points with concave bases, various stone tools such as choppers, scrapers, and knives. Dates: 2000 B.C.-A.D. 500.

Group II.-Pottery: Plain Wares (Alma Plain and Lino Gray). Dates: A.D. 500-700.

Group III.-Pottery: White Mound Black-on-White, Kiatuthlanna Black-on-White, and Red Mesa Black-on-White. Dates: A.D. 700900 .

Group IV.-Pottery: Reserve Black-on-White, Snowflake Black-on-

White, Wingate Black-on-Red, and Show Low Black-on-Red. Dates: A.D. 900-1100. 
Group V.-Pottery: Tularosa Black-on-White, St. Johns Polychrome. Dates: A.D. 1100-1300.

Group VI.-Pottery: Zuni Glazes, Pinedale Polychrome, Pinedale Black-on-White. Dates: A.D. 1300-1500.

\section{FIELD PROCEDURE}

Each site visited was given a survey number. A random collection of pottery and/or artifacts was made. The site was carefully clescribed as to its extent, condition, topographic-geographic setting, and spatial location. The pottery and artifacts were sorted and, where possible, placed into described types and counted. All the above information was entered on "Site Cards." The collections and cards for each site are available in the Department of Anthropology, Chicago Natural History Museum. The pottery counts, analyses, and counts of artifacts along with a description of each site found in 1959 and 1960 are published on micro cards (Martin, Rinaldo, and Longacre, 1960; Martin, Rinaldo, Longacre, and Freeman, 1961).

\section{TEMPORAL-SPATIAL OCGUPATION OF THE SURVEYED REGION}

The combined findings of the two seasons are outlined below on the basis of geographical location and the "group" affiliation of the components or sites. A total of 243 components was found, broken down as follows:

Little Colorado River Valley:

112 Components located:

11 Pre-pottery (Group I)

8 Plain Ware (Group II)

30 Early Black-on-White (Group III)

40 Reserve Black-on-White (Group IV)

19 Tularosa Black-on-White (Group V)

4 Zuni Glaze (Group VI)

"Triangle":

27 Components located:

11 Pre-pottery (Group I)

4 Plain Ware (Group II)

5 Early Black-on-White (Group III)

4 Reserve Black-on-White (Group IV ${ }^{\top}$ ) 
3 Tularosa Black-on-White (Group V')

0 Zuni Glaze (Group VI)

Snowflake-Mesa Redonda

67 Components located:

10 Pre-pottery (Group I)

2 Plain Ware (Group II)

16 Early Black-on-White (Group III)

26 Reserve Black-on-White (Group IV)

13 Tularosa Black-on-White (Group V)

0 Zuni Glaze (Group VI)

Show Low Area:

13 Components located:

5 Pre-pottery (Group I)

3 Plain Ware (Group II)

2 Early Black-on-White (Group III)

1 Reserve Black-on-White (Group IV')

0 Tularosa Black-on-White (Group V)

2 Zuni Glaze (Group VI)

White Mountains:

24 Components located:

0 Pre-pottery (Group I)

o Plain Ware (Group II)

7 Early Black-on-White (Group III)

14 Reserve Black-on-White (Group IV')

3 Tularosa Black-on-White (Group V')

0 Zuni Glaze (Group VI)

\section{SITE LOCATIONS}

\section{Key to Tables $14-20$}

Numbers refer to number of sites or components in the designated area, located in each topographic position.

Letters refer to the position of the site:

$A=$ on mesa top

$B=$ on "tier" or "bench" on side of mesa

$C=$ knoll or ridge on flat in valley

$D=$ on valley floor, on flat

$E=$ bluff or point of land jutting into the valley

$F=$ on knoll or hill in high area or uplands

$G=$ on shore of presently dry lake

$H=$ "cliff dwelling" 
TABLE 14.-GROUP I: PRE-POTTERY (2000 B.C.-A.D. 500)

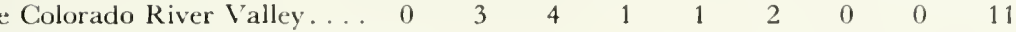

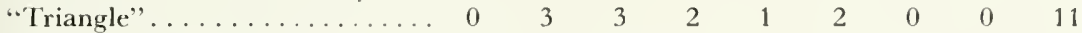

Snowflake-Mesa Redonda..... $\begin{array}{llllllllll} & 2 & 2 & 2 & 3 & 1 & 0 & 0 & 0 & 10\end{array}$

Show Low Area. . . . . . . . . . 1 . 0 . 0 .

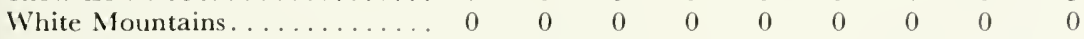

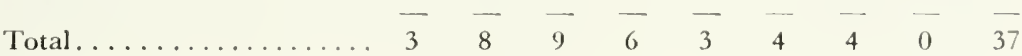

TABLE 15.-GROUP II: PLAIN WARES (A.D. 500-700)

$\begin{array}{lllllllll}\text { A } & \text { B } & \text { C } & \text { D } & \text { E } & \text { F } & \text { G } & \text { H Total }\end{array}$

Little Colorado River Valley. ...

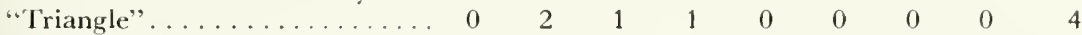

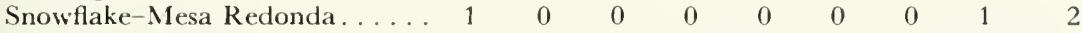

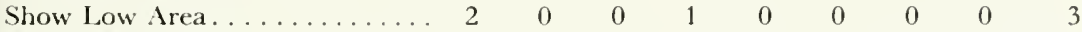

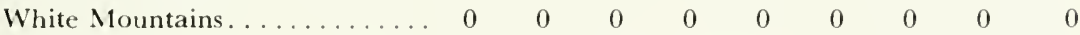

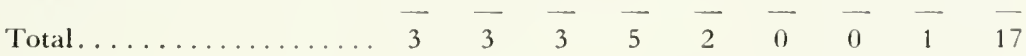

TABLE 16.-GROUP III: EARLY BLACK-ON-IVHITES (A.D. 700-900)

\begin{tabular}{|c|c|c|c|c|c|c|}
\hline & A & B & C & D & $\mathrm{E}$ & $\mathrm{F}$ \\
\hline Little Colorado River Valley.... & 1 & 1 & 9 & 9 & 10 & 0 \\
\hline "Triangle" . . . . . . . . . & 0 & 3 & 2 & 0 & 0 & 0 \\
\hline Snowflake-Mesa Redonda.. & 0 & 0 & 12 & 2 & 2 & \\
\hline Show Low Area......... & 0 & 0 & 1 & 0 & 1 & \\
\hline White Mountains...... & 0 & 0 & 5 & 0 & 1 & 1 \\
\hline Total. . & $\overline{1}$ & $\overline{4}$ & $\overline{29}$ & $\overline{11}$ & $\overline{14}$ & \\
\hline
\end{tabular}

'TABLE 17.-GROUP IV': RESERVE-SNOWFLAKE BLACK-ON-WHITE

(A.D. 900-1100)

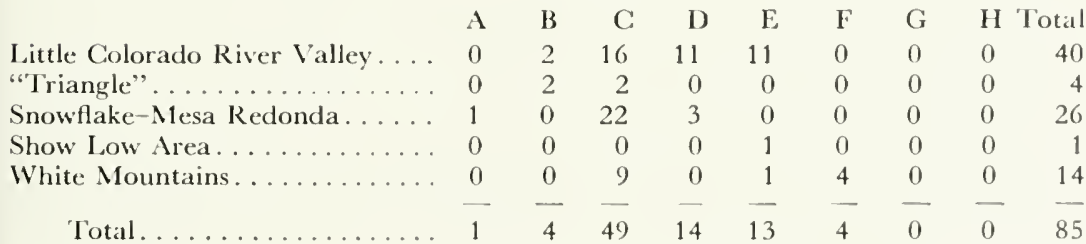


'TABE 18.--GROUP V: 'TULAROS.1 BL.ICK-O.N-WHITE (A.1). 1100-1300)

\begin{tabular}{|c|c|c|c|c|c|c|c|c|c|}
\hline & $\Lambda$ & 13 & C & D) & E & $\mathrm{F}$ & G & H & Total \\
\hline Little Colorado River Valley.... & 0 & 2 & 11 & 0 & 6 & 0 & 0 & 0 & 19 \\
\hline "Triangle" . . . . . . . . . . . . & 0 & 2 & 1 & 0 & 0 & 0 & 0 & 0 & 3 \\
\hline Snowllake-Mesa Redonda...... & 0 & 0 & 13 & 0 & 0 & 0 & 0 & 0 & 13 \\
\hline Show Low Area. .............. & 0 & 0 & 0 & 0 & 0 & 0 & 0 & 0 & 0 \\
\hline White Mountains... & 0 & 0 & 1 & 0 & 0 & 2 & 0 & 0 & 3 \\
\hline lotal. & 0 & 4 & 26 & 0 & 6 & 2 & 0 & 0 & 38 \\
\hline
\end{tabular}

TABLE 19. GROUP VI: ZUNI GLAZES (A.D. 1300-1500)

\begin{tabular}{|c|c|c|c|c|c|c|c|c|}
\hline & $A$ & B & C & $\mathrm{D}$ & $\mathrm{E}$ & $\mathrm{F}$ & G & $\mathrm{H}$ \\
\hline Little Colorado River Valley.... & 0 & 0 & 3 & 0 & 1 & 0 & 0 & 0 \\
\hline "Triangle" . . . . . . . . . . . . & 0 & 0 & 0 & 0 & 0 & 0 & 0 & 0 \\
\hline Snowflake-Mesa Redonda... & 0 & 0 & 0 & 0 & 0 & 0 & 0 & 0 \\
\hline Show Low Area............ & 1 & 0 & 0 & 0 & 0 & 0 & 1 & 0 \\
\hline White Mountains . . . . . . & 0 & 0 & 0 & 0 & 0 & 0 & 0 & 0 \\
\hline 'Total . . . . . & 1 & 0 & 3 & 0 & 1 & 0 & 1 & 0 \\
\hline
\end{tabular}

TABLE, 20.-IOCATIONAL PREFERENCES FOR THE ENTIRE, SURVEYED AREA

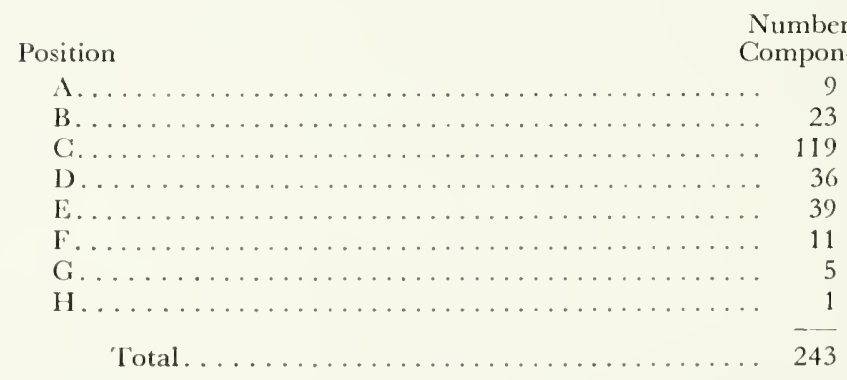


In summary, the pre-pottery peoples in this region lived high up on the sides of mesas on "tiers" or "benches," on knolls or ridges down in valleys, or on the flat bottom lands. The earliest pottery-making peoples (Group II) lived on the valley floor or on knolls in the valley, on "tiers" on the sides of mesas. or on mesa tops. The people making the earlier black-on-white pottery (Group III) show a definite preference for knolls or ridges down in the valleys as favorable sites for their villages. as do the later peoples in the area (Group IV through Group VI). Out of a total of 243 components found in the region, 119 were located in this topographic position, the home-site definitely preferred by the prehistoric inhabitants of the area.

\section{GENERAL SUMMARY}

The earliest cultural evidence for the occupation of the area consists of open sites, probably the scene of impermanent camps. indicated by a scattering of stone tools. These sites occur along the shores of presently dry lake beds, on knolls and ridges down in valleys, and high up on flat areas on the sides of mesas. Two sites, both located on the beaches of playas, were excarated by the Museum in 1958 (Martin and Rinaldo, 1960a). Martin and Rinaldo report a C14 date for one of these sites (Laguna Salada) as ca. 3200 years ago. The other site (Little Ortega Lake) may be older (op. cit., p. 115). No indications of permanent architecture were found.

The artifacts found at the Beach Sites (op. cit., pp. 12-34) are somewhat similar to those which were collected on the surface reconnaissance. Many of the sites had basin-type metates and pebble-type, one-hand manos on the surface. In addition, quantities of stone tools, bifacially worked scrapers, flake knives, and projectile points were collected. The most sensitive indication of the cultural affiliations of this pre-pottery material appears to be the projectile points. A wide range of forms was found, but the presence of stemmed, indented-base types at many of the sites provides some clue as to the affinities. This particular style of projectile point evidently has a wide distribution over the Southwest. It appears to be associated with the Desert Culture (Jennings, 1957) and is reported at a number of early sites. The general style of the point has been ascribed names by several authors reporting on widely separated locations: The Pinto Point (Campbell and Campbell, 1935, pl. 13), San Jose Point (Agogino and Hester, 1956, pp. 9-12), and Amargosa II Points (Haury, 1950, fig. 61). Those found at Tularosa Cave (Martin, et al., 1952, p. 497) are also examples. 
The pre-pottery material in this area of eastern Arizona has been described as the Concho Complex (Wendorf and Thomas, 1951), based on surface collections at a number of sites. Wendorf and Thomas ascribe the majority of the Concho materials to the California desert cultures with closest aflinities to the Amargosa II component at V'entana Cave and the Pinto Basin materials in California (op. cit., p. 107). Martin and Rinaldo, basing their suggestion on the assemblage of implements found at the Beach Sites, feel that the material has affiliations with those described for the San Jose, Concho, and Cochise industries (Martin and Rinaldo, 1960a, p. 115).

The material collected during the past two field seasons by the Southwest archaeological expedition of the Museum can add little to this description. The sites are relatively small in area, ranging from 100 square feet to several thousand square feet, and they occur sporadically thoughout the surveyed area. The implements suggest an economy based upon collecting wild plant foods and hunting. This generalized Desert Culture pattern seems to persist until some time after the beginning of the Christian era.

Several qualifications are mandatory before presenting a description of lithic materials from the pre-pottery sites.

First, almost all the pre-pottery sites in the sample have been visited by local collectors over a period of years. Numerous trips to these sites by collectors have resulted in rather large collections, and this fact may possibly explain the relatively small number of projectile points and other stone tools in our collections. For this reason, our sample of the surface materials from these sites is incomplete.

Second, an increasing understanding of the material aspects of the Desert Culture is a result of the excavation of dry caves in the Basin area. Danger Cave (Jennings, 1957) is a prime example. We are beginning to appreciate the role of stone tools in the assemblage. Unfortunately, the surface sample for the surveyed area is restricted to open sites from which we have only lithic material. The inadequacy of this sample (see above) indicates a serious sampling error which is compounded by the very nature of the minute fragment of the total material culture that our collections encompass. Jennings (op. cit., p. 279) pointedly describes the problem when he suggests that ". . . flint was cheap, expendable and unimportant, whereas cordage, basketry, buckskin, bone and horn tools, handles, arrows all represented greater skill, a greater expenditure of effort, and had actually a higher practical and investment value than did the stone. If flint were thus cheap one wonders how important it was. How valid are detailed reconstructions of culture history based on flint typology?", 
Thus, on at least two counts, our sample is handicapped. In the face of this problem, artifacts were classed into rather broad functional classes: manos, choppers, projectile points, scrapers, and so on. These were further divided on the basis of shape, size, and technique of manufactor. The results are summarized (figs. 69-74). For a more comprehensive view of the pre-pottery lithic materials of the Concho Complex, see Martin and Rinaldo (1960a, pp. 12-34), Wendorf and Thomas (1951), Thomas (1953), Breternitz (1957).

By A.D. 300 several important changes evidently had occurred in the area. Settlement was somewhat more permanent. One site, the Tumbleweed Canyon Site, was excavated by the Museum in 1960 (see Chap. I, p. 19). Three shallow pithouses and three associated storage pits were dug. The site was on an easily defended mesa with boulder walls augmenting the natural defensive character of the location. The paleopollen analysis (Chap. VIII) indicates that these people had corn, although it is felt that the corn served as an augmentation to the wild plant foods gathered in the area, rather than a staple. Slight changes in the stone artifacts are also noted. The manos and metates are, in general, similar to those found on earlier sites. The projectile points show the preference of these people for notched forms. The remainder of the stone material is somewhat similar to that reported from the earlier sites with one exception, the presence of a new tool, a pestle found in one of the houses (Chap. VI, p. 122).

Several pre-pottery sites located by the survey showed evidence of rock-outlined pithouses. The lack of any sensitive indication that these sites are as late as the Tumbleweed Canyon Site, compounded by the fact that when these houses do occur they are associated with early sites of the general Concho Complex, leaves us in a quandary as to the numbers and nature of these relatively late pre-pottery sites. No parallels to this situation could be found in the literature. There are some rough similarities between the Tumbleweed Canyon Site and the Flattop Site (Wendorf, 1953) such as the small size of the houses and the presence of basin metates, and some resemblance in the chipped stone artifacts found at the two sites, but the differences (presence of pottery at the Flattop Site as well as lateral entrances to the houses, rectangular manos and troughed metates) appear to outweigh the similarities. It is interesting to note that these two sites are located in the same drainage (the Little Colorado River) and are probably more or less contemporary.

About 300 years after the occupation of the Tumbleweed Canyon Site, the area was occupied by pottery-making people who lived in pithouse villages. At this time, two types of pottery were introduced as part of 


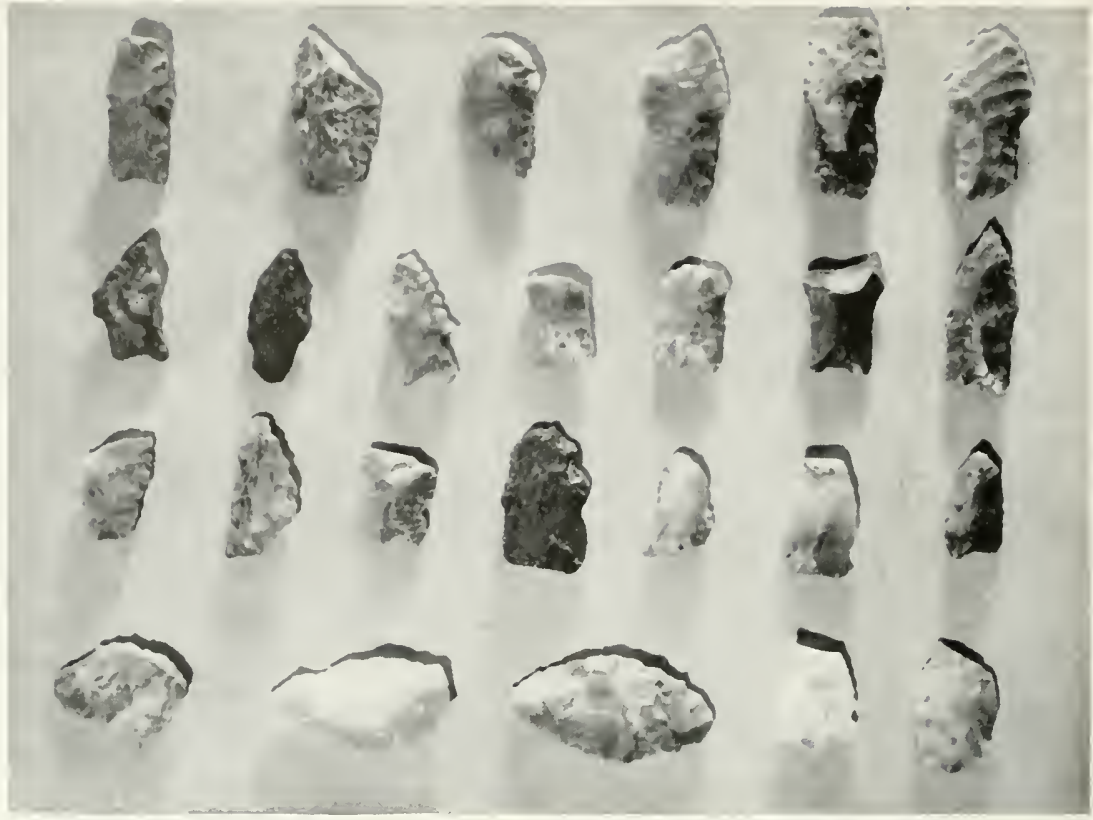

Fig. 69. Projectile points from pre-pottery sites. Point in upper left (broken) $2 \mathrm{~cm}$. long.

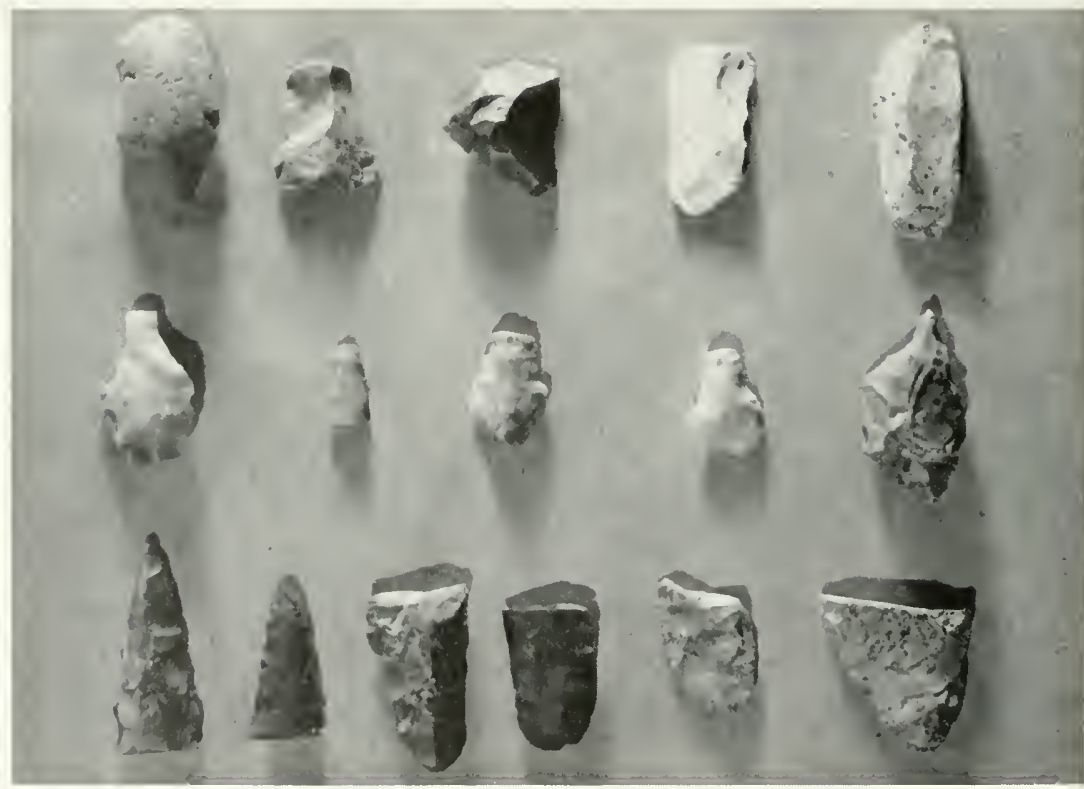

Fig. 70. Miscellaneous tools from pre-pottcry sites. Top row, left, scraper (concave-convex in cross section), $4.2 \mathrm{~cm}$. long. Remainder of top row, flake knives. Middle row, drills or perforators. Bottom row, blade fragments. 
two distinct cultures. One group of people, with cultural ties north of the area, was making an unslipped gray ware (Lino Gray), and another

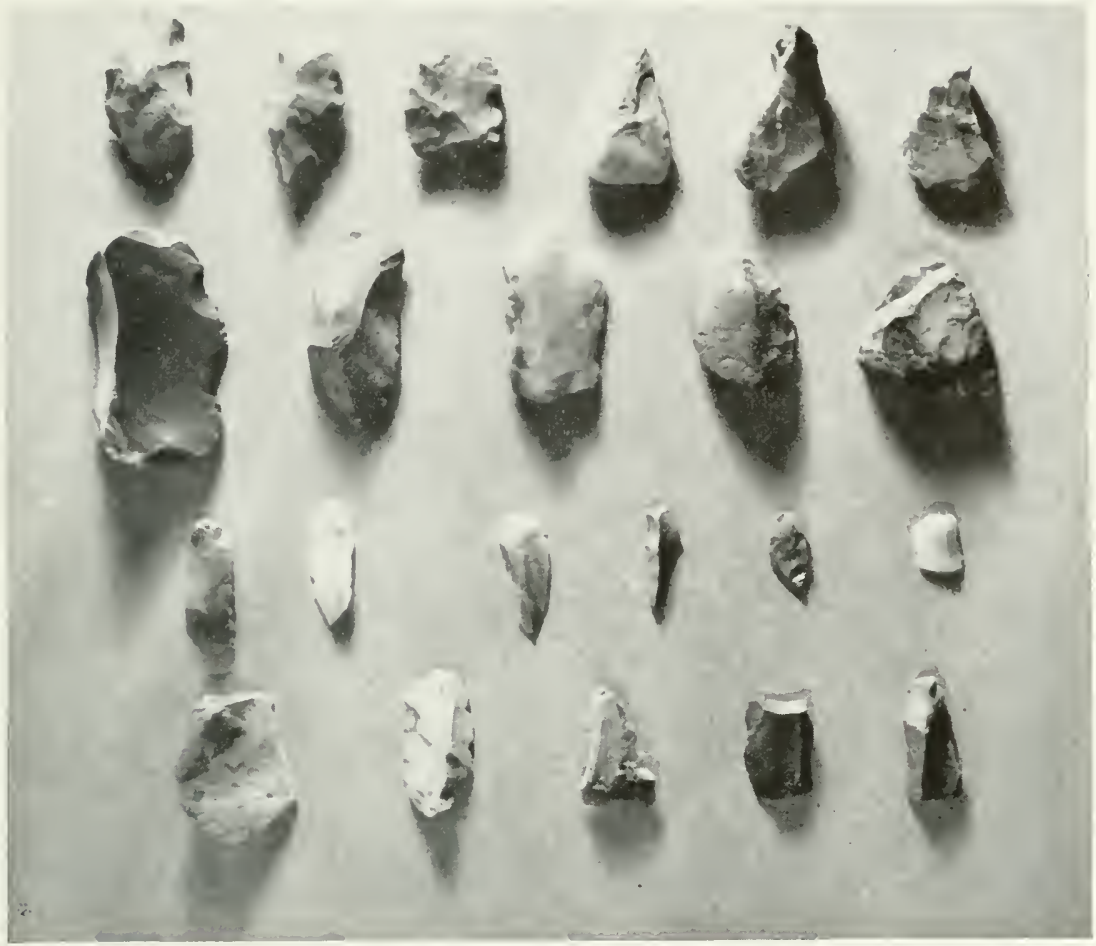

Fic. 71. Scrapers and utilized flakes from pre-pottery sites. Upper left, scraper $3.5 \mathrm{~cm}$. long. Top row, three on left, bifacially worked scraper knives; three on right, end scrapers. Second row, side scrapers. Bottom two rows, utilized flakes.

group was making an unslipped brown ware (Alma Plain). In many respects these two cultures shared a similar way of life. Both groups lived in small pithouse villages containing from three to ten or more houses each. They were agricultural peoples, probably by this time depending for most of their food on their own crops augmented by natural plant foods and the products of hunting.

The villages of the people utilizing the gray pottery were concentrated in the northern and eastern portions of the region. The sites yielding brown pottery were concentrated in the northern and southern reaches of the survey. On most of the sites with Lino Gray pottery, traces of Alma Plain appear as intrusive trade pottery, and the Brown Ware sites show traces of Lino Gray. 


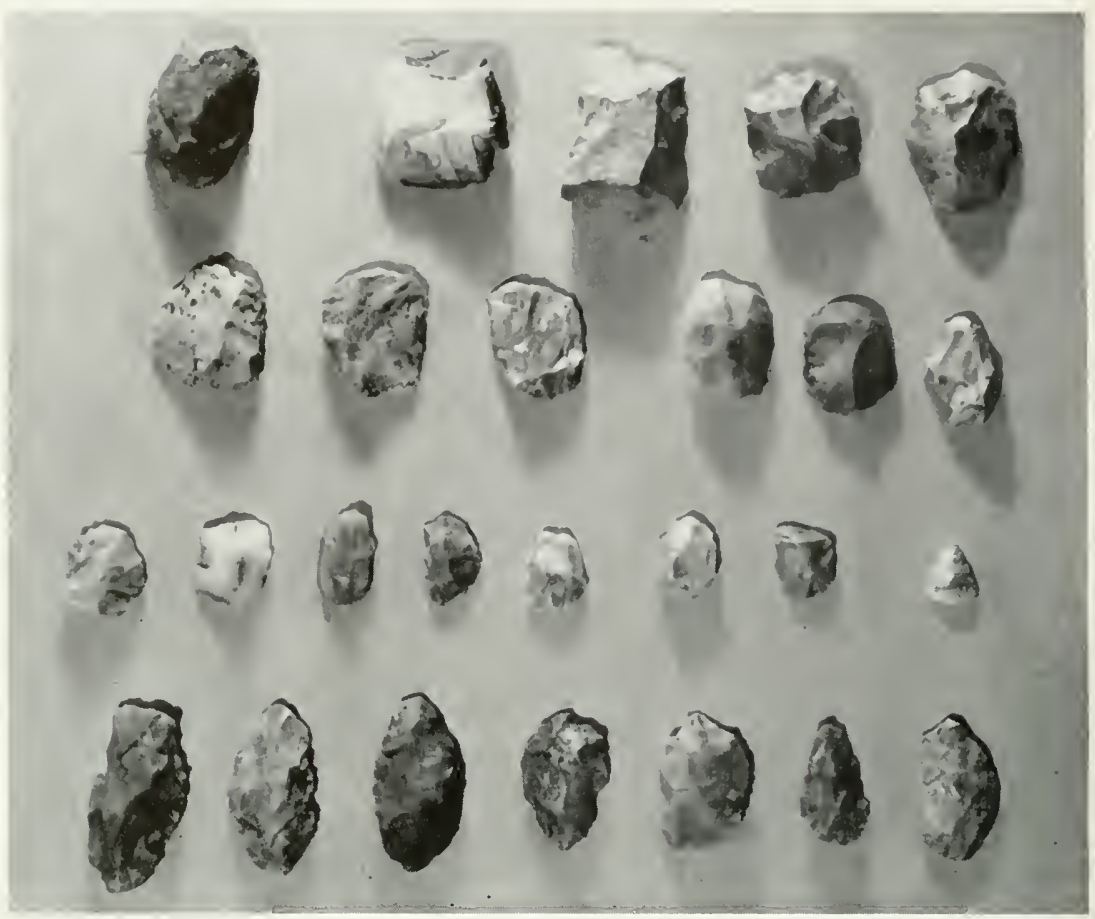

Fig. 72. Scrapers from pre-pottery sites. Specimen at upper left, $4 \mathrm{~cm}$. long. Top row, plano-convex scrapers or "pulper-planers." Remainder, bifacially worked scrapers showing variation in size and shape.

The following occupations of the area are not as easily understood as the above. It is felt that the people who made the brown pottery might represent either a direct migration from the Mogollon centers to the south and east, and consequently represent a hybrid culture, or more probably mirror strong Mogollon influence as the result of culture contact and acculturation on the part of indigenous populations. The people who made the gray pottery seem to be closely allied with the Anasazi to the north.

The period beginning at approximately A.D. 700 is characterized by an increase in both the number of sites found and the individual size of each site. It is felt that this may be an indication of a population increase which was relatively sudden, and judged from the nature of pottery and other artifacts from sites occupied at this time. was markedly Mogollon in character. From this time on, in fact, the nature of the prehistoric occupation of the area is dominantly Mogollon. Brown utility wares are found from this period through the latest sites we record in the region. 
It least in the eastern portion of the surveyed area, the painted pottery is elosely allicd with the painted series worked out for the Mogollon in Pine Lawn Valley by Martin and Rinaldo. The analysis of stone artifacts appears to substantiate the pottery evidence.

Through time, the sites became larger, the pottery and artifacts more complex and sophisticated, and the people more numerous. By A.D. 1200, a significant bifurcation of culture appeared within the limits of the surrey. In the east, especially in the valley of the Little Colorado River, the Mogollon tradition was culminating in very large pueblo villages. some with pan-rillage or inter-village rectangular Great Kivas. The pottery was colorful. some decorated with glaze paints. One can follow a Mogollon sequence in design style in an unbroken line from some of

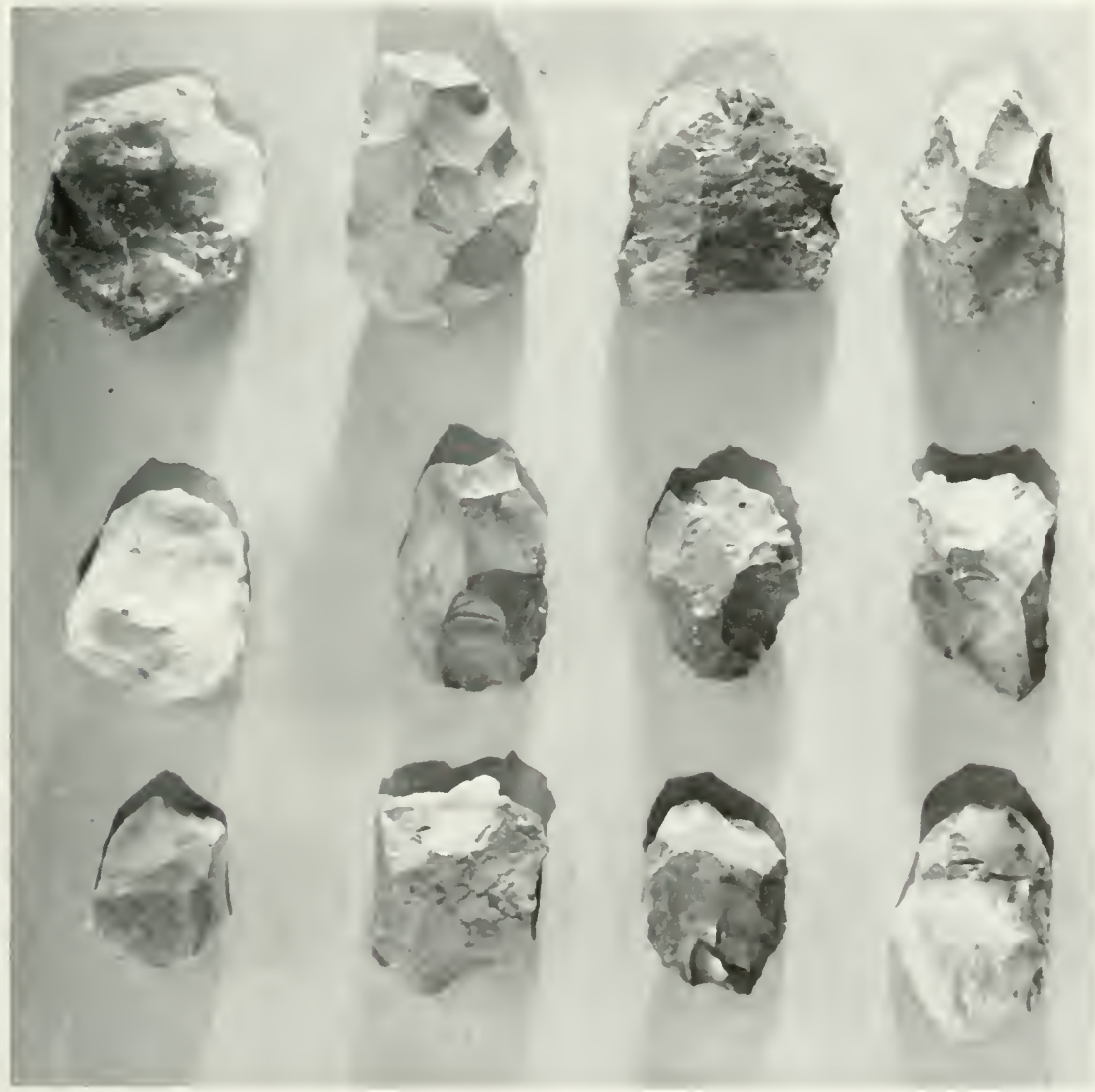

FIG. 73. Large bifacially percussion-flaked scrapers/choppers from pre-pottery sites. Specimen at upper left, $6.5 \mathrm{~cm}$. in length. 

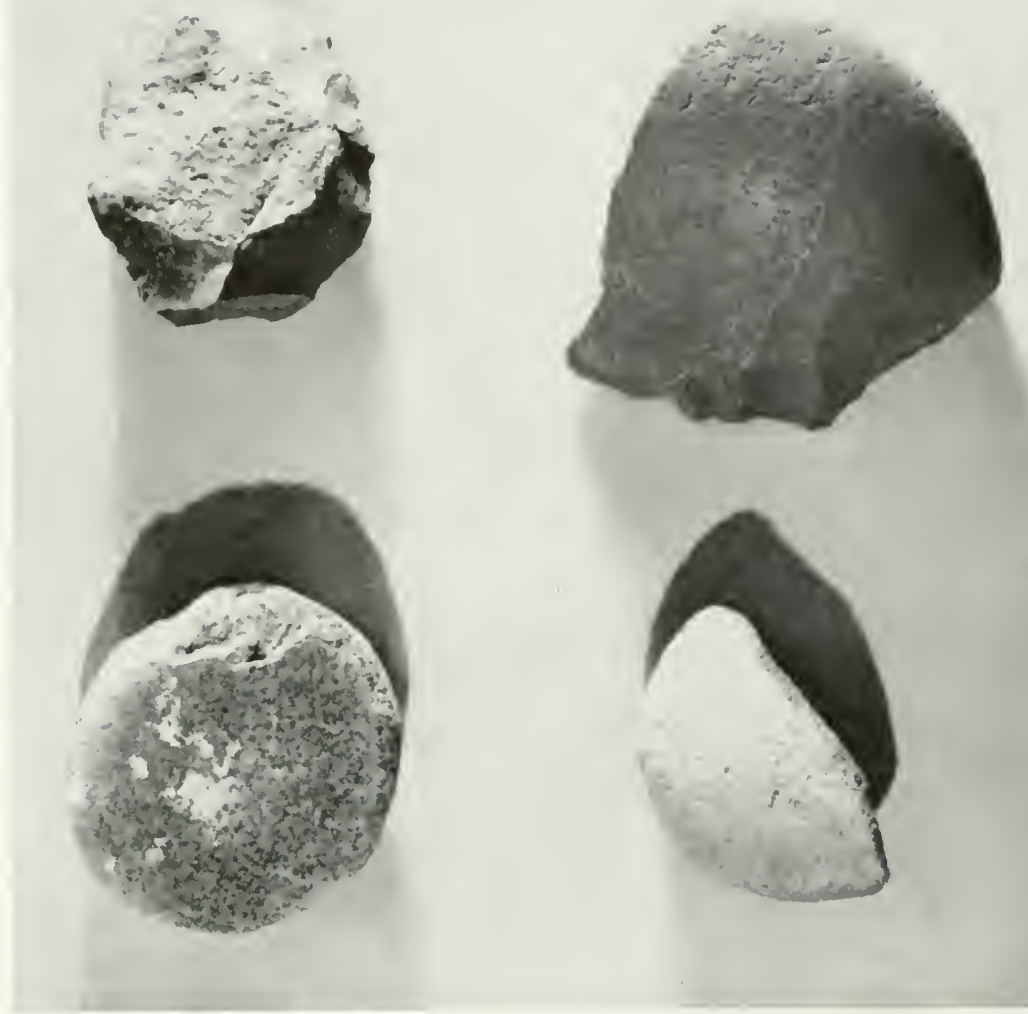

FIG. 74. Choppers and manos from pre-pottery sites. Top row, ehoppers. Bottom row, pebble-type "one-hand" mano and mano fragment. Specimen at upper left, $8.5 \mathrm{~cm}$. long.

the carliest black-on-white styles through the glazes. In the western portion of the area, however, a somewhat different pattern seems to emerge. Here, centered in the area north and west of Mesa Redonda, near the modern town of Snowflake, cultural remains reflect differences in the ceramics as well as major differences in ceremonial architecture compared to those found in the Little Colorado River Valley. Circular Great Kivas make their appearance in this locale-the only time and place in the entire surveyed area with one exception. A circular Great Kiva was excavated by the Museum at the Mineral Creek Site in 1959 (Martin, Rinaldo, and Longacre, 1961). The circular Great Kivas located near Snowflake are associated with large pueblo villages, just as 
their rectangular counterparts are in the east. The circular kiva seems to be an Anasazi trait. Thus one is faced with the problem of explaining the circular kiva among a people who seem in other respects to be strongly Mogollon in nature. The dominant decorated pottery type at this period, also, does not seem to fit into a Mogollon pattern. Here, design elements made up of balanced and opposed solids are most abundant in the ceramics. In the eastern portion of the surveyed region at roughly the same time, the Mogollon Reserve-Tularosa series with design elements made up of solids and hachured scrolls is the preference. Small quantities of intrusive Anasazi trade pottery (Tusayan White Ware), almost identical in design style with the locally made pots, were recovered on sites in the Snowflake region. This fact, along with the architectural evidence, leads one to suspect Anasazi influence in this small portion of the surveyed area. The presence of Mogollon brown utility wares in this area in conjunction with the seemingly Anasazi nature of much of the Snowflake sub-culture is one of the many intriguing complexities that must be explained.

\section{CONCLUSIONS}

My task now is to present an interpretative synthesis for the sequence of prehistoric events in this region.

The earliest occupants of this area appear to have been small groups of nomadic intensive collectors. Primary subsistence of these groups was probably based upon collecting wild plant foods augmented by hunting. The known material culture indicates a generalized Desert Culture adaptation to the enviromment. The distribution of sites may indicate a seasonal round of settlement, probably based upon the harvest cycle of various plant foods. I would suggest that the open unsheltered sites might have been occupied during the warmer months, probably utilized for short periods of time in the collecting activities of these groups.

Location of these sites probably depended upon availability of water and proximity of a food source. Examples of these locations would be the sites in the lower elevations of the area such as the Beach Sites. More sheltered locations such as those sites at the bases of cliffs or on "tiers" on the sides of mesas may represent sites occupied cluring colder periods. The figures on site locations (p. 154) indicate roughly three times as many sites in a rather umprotected spot as those which incorporate natural shelter in their location. This would be the expected pattern in the seasonal round. Regular movements to new collecting areas as harrests ripened, probably returning to favored camp areas, would result in a number of frequented sites occupied during the collecting season. Fewer 
naturally sheltered sites may simply reflect the same population in a smaller number of stable wintering camps. Evidence for the periodic occupation of a favored site can be seen in the several styles of projectile points present at each of the excavated Beach Sites (Martin and Rinaldo, 1960a, pp. 24-29). These types may represent the style preferences in projectile points in different periods of time among the people utilizing the beach locations as camping spots. One might expect such style changes, considering the fact that the periodic occupation of such favored sites probably took place over a period of several hundreds of years.

Apparently none of the sites of this early period was selected with an eye to defense. Clearly most of them are in unprotected locations which would be most difficult to defend. The sites which are high on the sides of mesas were set against the base of a cliff. In all cases, the the tops of these cliffs are easily accessible. Several large rocks dropped from above would underline the indefensive nature of such locations.

This pattern of settlement location is not shared with sites of this early time period in neighboring areas to the east and southeast. Danson (1957, p. 103) reports a defensive character for "pre-Pueblo I" sites in the area east of the Vernon region. Bluhm (1960, p. 541) reports a similar pattern in her summary of the settlement patterns of the Pine Lawn area.

The combined data of settlement pattern and material culture suggest some sort of band socio-political organization for the peoples of this period. If my speculation on the seasonal round of collecting for these groups is well-founded, I may then suggest a system of defined territoriality for each social group. Perhaps there was economic co-operation among bands in such things as large seed harvests or rabbit or antelope drives.

This Desert Culture pattern proceeded with great continuity for at least two thousand years. The earliest date for material in our area is ca. 1550 B.c. It is probable that this material has even greater antiquity. The pattern continues until some time after the the birth of Christ. The earliest date thus far on major modifications of this generalized Desert Culture Base is approximately A.D. 275. These major modifications are: the beginnings of agriculture in the area, the first appearance of houses, settlement definitely oriented toward defense in one part of the region, the development of preserving and storage techniques, and relatively permanent settlement. This period of great change is exemplified in the Tumbleweed Canyon Site.

We feel that these changes reflect the acceptance of foreign exploitive-adaptive methods for subsistence on the part of the indigenous peoples. These techniques probably were introduced from the Mogollon 
area to the south or southeast. The beginnings of agriculture (raising corn, probably along with beans and squash), combined with the technical knowledge of preserving and storing surplus foodstuffs, would have allowed relatively permanent settlement and an increase in population, and would have increased the time available to both sexes as their production techniques were refined. An agricultural base would also have provided the aged and young with a role in food production not present for them in a collecting-hunting economy. This would have appeared in their usefulness in tending the growing crops, in the harvest, and in preparation of the foodstuffs for storage.

In the Tumbleweed Canyon site we have probably captured these far-reaching changes in a relatively early portion of their introduction. The people who occupied the site probably still depended heavily upon natural plant foods for their subsistence. The assemblage at this site reflects close ties with the material culture of the Concho Complex. In these respects the data from the Tumbleweed Canyon site suggest great continuity with the past. In the additions to this Concho Complex Base can be seen the complex changes foreshadowing the later prehistory of this particular area of the Southwest.

The defensive nature of this site presents a problem of explanation. The defenses were much too elaborate to be explained by a fear on the part of these people of wild animals robbing their stored foodstuffs. It may be better explained by a fear of other people. This, of course. raises the problem of identifying these unknown peoples. There are two possibilities: These people are either local groups, similar to those who occupicd Tumbleweed Canyon, or they are people from somewhere outside the area.

The data I have at present suggest that the unknown people referred to above were not local and that the defensive nature of the Tumbleweed Canyon Site was in response to foreign peoples. If I assume that sites of this period are those with small, rock-outlined pithouses and lacking pottery, then there are very few sites and a small population. All other sites with pre-pottery pithouses that have been located thus far have not been defensive in nature. They occur on locations of earlier Concho Complex sites. If I postulate a group of liberal people accepting such radical innovations as corn agriculture and permanent settlement, surrounded by conservative groups maintaining a Desert Cultural pattern with local friction aroused to a point of conflict, it is difficult to explain the non-defensive aspects of these other sites.

The very presence of exploitive techniques foreign to this area indicates contact on some level with outside peoples. The suggestion that these 
mnknown peoples were from some other area raises the problem of the nature of this encroachment. Were they moving into the area to establish villages? No evidence was discovered that would indicate foreign peoples were settling in the area at this time.

Do we have evidence suggesting that foreign peoples were passing through the area? The evidence at hand does suggest that the defensive nature of the Tumbleweed Canyon Site was in response to a threat presented by exotic peoples passing through the area and utilizing the Little Colorado River Valley as a natural route of travel. This hypothesis is supported by the non-defensive nature of the other sites of this period.

All are located away from the valley itself, in the area designated as the "triangle" (Martin, Rinaldo, and Longacre, 1960, pp. 174, 176). These sites are far from the major route of travel that the valley was to become and probably was, to an extent, by this early period. The threat presented by outsiders passing through would not have been felt by the groups removed from the valley itself, but they were able to benefit from the innovations that such outside contact provided. The advantages of living on the edge of a well-watered and fertile valley such as the Little Colorado Valley probably was, evidently outweighed the negative aspects of such a location.

After this inital period of new exploitive techniques, the peoples of the area began to add new items to their culture and expand and refine older techniques. Deeper and larger pithouses were constructed, the art of ceramics was learned, and expanding agriculture allowed for an increased population.

For the first several hundred years after the Tumbleweed Canyon Site was occupied, both Anasazi and Mogollon ideas were filtering into this area. By A.D. 800, however, the pattern began to assume an everincreasing Mogollon character which it never lost.

By A.D. 800, fairly large pithouse villages were in the region, with a firm agricultural base. By A.D. 1000, population had greatly increased and some peoples were living in above-ground pueblo units. The next several hundred years saw the number of villages decrease, but the individual size of each greatly enlarge. This pattern continued, eventually culminating in the large pueblos in the region by A.D. 1350. Beginning about A.D. 1000 new settlements were made only on major waterways such as the little Colorado, and the rugged portions of the region such as the "triangle" were abandoned. By A.D. 1350, the only permanent settlements in the area were either along the Little Colorado itself, or Silver Creek. one of its tributaries. 
There are many questions that could be raised, but at this stage in our knowledge they are not answerable. Future archaeological work in this region should clarify such problems, many of which we do not even recognize. We shall have to refine the chronology and enlarge our comprehension of the cultures of the area in order that we may be able to seek answers to such basic questions as: What is the role of this region in the contacts between the Mogollon to the south and the Anasazi to the north? What significance does this area hold for an understanding of the late development of Zuni and Hopi cultures? Is there any information in this sequence which may aid our understanding of the Salado Culture to the south and southwest? 


\title{
VIII. The Pollen Analysis of Eighteen Archaeological Sites in Arizona and New Mexico
}

\author{
By James SCHOENWETter \\ Department of Anthropology, Southern Illinois Lniversity
}

ACKNOWLEDGMENTS

This project was initiated through funds given by the National Science Foundation (Grant No. G13039).

The Geochronology Laboratories of the University of Arizona allowed me use of the facilities of its palynological laboratory and gave generously of the time and experience of its staff in checking the results and interpretations presented in this report. To Professor T. L. Smiley and Dr. Paul S. Martin of this laboratory grateful thanks are tendered for the supervisory roles they have accepted.

The Department of Botany of Southern Illinois University, through the generosity of its chairman, Dr. Walter B. Welch, lent a research microscope to the project.

The Department of Anthropology of Southern Illinois University allotted office space and facilities which made the progress of the research a far smoother operation than might otherwise have been the ease. To the members of the staff of this department and to the members of the staff of the Museum at Southern Illinois Lniversity, especially Dr. Walter W. Taylor and Professor P. Armillas, I wish to express my appreciation for their patience in reading this report in manuscript and for their courteous and helpful suggestions.

\section{IN'TRODUC'TION}

The archacologists' interest in pollen analysis stems from their recent tendency to place greater emphasis on the relationships between culture and environment when analyzing prehistoric remains. This has particularly stimulated palynological research, which has been carried on in association with archaeological research in the arid Southwest over 


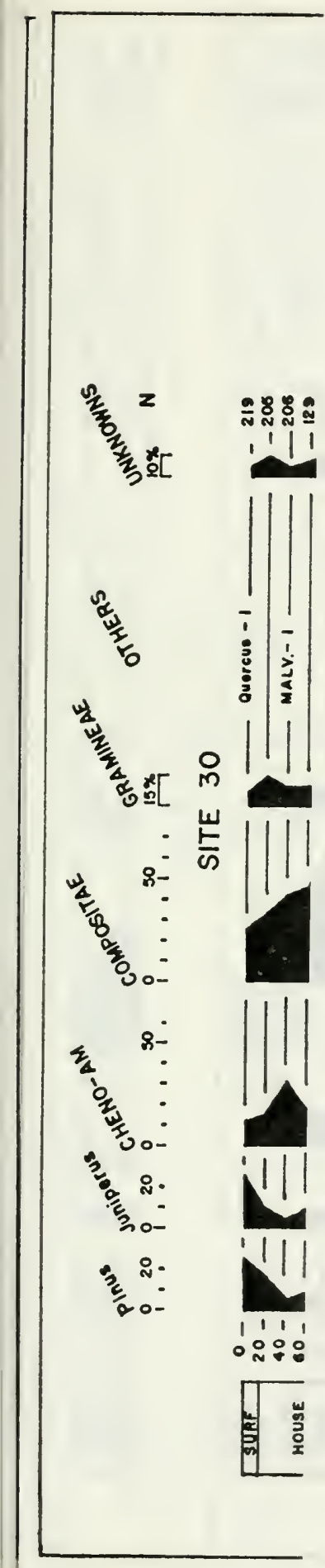


the past few years. The results of some such pollen studies, however. are as yet unpublished, since they can best be interpreted only after thorough analysis of the accompanying archaeological materials. When this project was being formulated it was realized that pollen studies undertaken upon archaeological sites which were already well described would yield information which could be more quickly disseminated and perhaps most relevant to the question of the nature of the relationships between culture and environment.

Over the past twenty years Chicago Natural History Museum has excavated and reported upon an impressive number of sites in eastern Arizona and western New Mexico. These sites encompass a respectable span of time and allow a rather substantial basis for discussing the single culture they represent: the Mogollon. It was natural, then, to attempt the pollen analysis of such well-known sites as the SU Site, Wet Leggett arroyo, the Promontory Site and Higgins Flat Pueblo in the Pine Lawn. New Mexico, area; and also to investigate Site 30. Table Rock Pueblo and others in the area around Vernon, Arizona.

The major objective of this research project was the development of a pollen chronology for the eastern Arizona-western New Mexico area north of the Mogollon Rim. We hoped that this would date and allow a comprehension of such environmental fluctuations as may have occurred. This objective has been essentially fulfilled, for the pollen analyses of many archaeological sites showed sufficient regularity to support the construction of such a pollen chronology. A close degree of fit can be shown between this chronology and other pollen and dendrological chronologies from the arid Southwest. When the pollen chronology is fully developed, a valuable stratigraphic tool will be available to the archaeologist interested in this region.

The final objective of the study was the investigation of whatever aspects of the relationship between culture and environment were made manifest in the course of the work. As an adequate reconstruction of the environment of the area investigated can be made for different time levels, a discussion of the relationships between the environmental changes evident in the pollen record and the cultural changes evident in the archaeological record will be presented. It is recognized that this is an interpretation of the evidence, but the result is considered as a testable hypothesis for future research.

Some of the interpretations in the following pages may be challenged, and further work may uncover errors, gaps and misorientations. I have attempted to demarcate clearly evidence, conclusions from the evidence, and interpretations based on these conclusions. However, I 
hope that the work will be regarded only as a step forward. Comments, suggestions and, especially, future development of palynological research along these lines are welcome.

\section{METHODS AND TECHNIQUES}

\section{SAMPLING}

As pollen analysis is a relative, rather than an absolute, dating technique, it was first imperative to collect sediment samples which could be dated with some degree of certainty. Most of the archaeological sites could be, or had already been, absolutely dated by radiocarbon, tree-rings, or ceramic typology. When test pits were dug into a site of known date, sediment samples could be collected which were assumed to be of the same age as the period of construction generally recognized for the site. It was necessary to collect the samples from previously unexcavated rooms or areas, however, to insure the collection of undisturbed sediments.

To increase the likelihood of gathering sediment samples which dated from the period of construction, samples were taken from the floors of rooms in the sites. A stratigraphic series of samples was then taken from the profile of the test pit, and such sub-floor features as pits were also sampled. This technique yielded one sample which could be regarded as the same age as the site (the floor sample), a series of samples which could be regarded as younger than the date of site construction by an unknown number of years (the fill samples), and one sample which might date either from the period of construction or some time earlier (the sub-floor sample).

After archacological sites of known date had been sampled, some sampling was also undertaken on newly surveyed, unexcavated, archaeological sites. Since these could be roughly dated by associated pottery types, they served to fill in certain gaps in the chronology. In this report the initials "LS" precede the survey number given to sites of this type. They are identical with those described by Longacre (Martin, Rinaldo, and Longacre, 1960; Martin, Rinaldo, Longacre and Freeman, 1961).

Sediment samples were also collected in stratigraphic sequence from the banks of arroyo cuts. In some instances these arroyo sites were selected for their provenience to archacological materials; in other instances it was hoped that though the samples were undated they could be tied into the dated series and would serve as a control that was uncontaminated by "cultural preference" for certain pollen types. 
A few samples were also taken from the muck or silt in cattle (stock) tanks. The open water surfaces of tanks act as natural pollen traps for the surrounding floral complex (Martin. Schoenwetter and Arms, 1961). Pollen recovered from such locales can be considered representative of the ecological conditions in the immediate area.

The total number of samples collected during the summer of 1960 was 263. Samples from five arroyos accounted for 100 of these-four arroyos in the Vernon. Arizona, area and one in the Pine Lawn, New Mexico, area. Four samples were collected from cattle (stock) tanks. Of the 159 samples taken from archaeological sites, 35 were from five sites in the Pine Lawn area and the rest were from 13 sites in the Vernon area.

Sampling technique was based on the principle that stratigraphic information was of vital and primary importance. The major limiting factor in palynological sampling is that pollen grains are microscopic and easily wind-blown. Efforts must therefore be made to avoid contamination of the samples by recently disseminated pollen or ancient pollen from other samples. Under ordinary field conditions it is nearly impossible to avoid every source of contamination, but if reasonable caution is exercised large amounts of contamination are not expected and small amounts will not alter main conclusions drawn from the data. The sampling technique used in the present study may help to guide other workers, and so is described in detail in Appendix A (p. 206).

\section{Extraction}

The procedure followed for extracting fossil pollen was that in general use at the Geochronology Laboratories of the University of Arizona for post-glacial arid land sediment samples. Because of time limitations, and because the types of sediment involved varied widely in texture, amount of organic material, etc., it was deemed unprofitable to experiment with a selected series of samples to determine the best extraction technique or techniques that could be utilized. In the interests of expediency and uniformity, all of the samples were processed by the same technique.

The extraction technique consists of three basic steps. In the initial step, the non-silicious fraction of the sample is separated from the silicious fraction (Arms, 1960). In the second step, the non-silicious fraction is subjected to a series of well-known procedures which dissolve much of the organic and non-organic materials from the pollen-bearing matrix (Erdtman, 1954; Facgri and Iverson, 1950; Wodehouse, 1935). In the final step, that fraction of the matrix which has a lighter specific 
gravity is captured by flotation (Frey, 1955) and is then prepared for viewing under the microscope. In Appendix B (p. 207) the extraction technique is described and discussed in greater detail.

\section{Analysis}

Though some of the samples resulting from the extraction process were given cursory inspection at the Geochronology Laboratories to ascertain that pollen had been recovered, the bulk of the analysis was undertaken at Southern Illinois University. This involved preparing and examining the microscope slides, recording their contents, and preparing the text and figures of this report.

Certain limitations affected this phase of the project. No laboratory facilities were available to process the samples. Therefore, samples which needed further chemical treatment before their pollen content could be adequately evaluated had to be ignored. Sometimes the amount of pollen that could be found on a slide was so small that many slides had to be examined before a statistically useful count was accumulated for a given sample. It seemed best to analyze first those samples which were least difficult to work with; then to go back, if necessary, to the more difficult ones to fill in such chronological or spatial gaps as were found. Since most of the samples from archaeological sites were productive of pollen, it was decided to concentrate upon them rather than upon the arroyo samples.

A group of ten sediment samples was analyzed by the Geochronology Laboratories as a check on my counting and identification. Their results were generally consistent with mine, though some differences did occur. It was found that counts made by the Geochronology Laboratories tended to show greater amounts of arboreal pollen, while my own tended to find greater frequencies of economic pollen, especially Cleome. In major pollen features, such as the dominance of cheno-am pollen, etc., however, the counts were in agreement.

Counting and identification of pollen was done at 440 magnifications. A count of 200 pollen grains from each level, exclusive of unknowns, was the goal but it could not always be obtained without a great deal of difficulty. The 200-grain count was selected because its statistical reliability has been investigated for arid land sediments (Martin, Schoenwetter and Arms, 1961), but counts between 150 and 200 grains were also considered usable.

The figures accompanying this report are of two types. Figure 75 is a composite pollen diagram showing the results of the pollen counts from a group of archaeological sites in the Vernon, Arizona, area, with the youngest at the top and the oldest at the bottom. Figure 76 shows the 


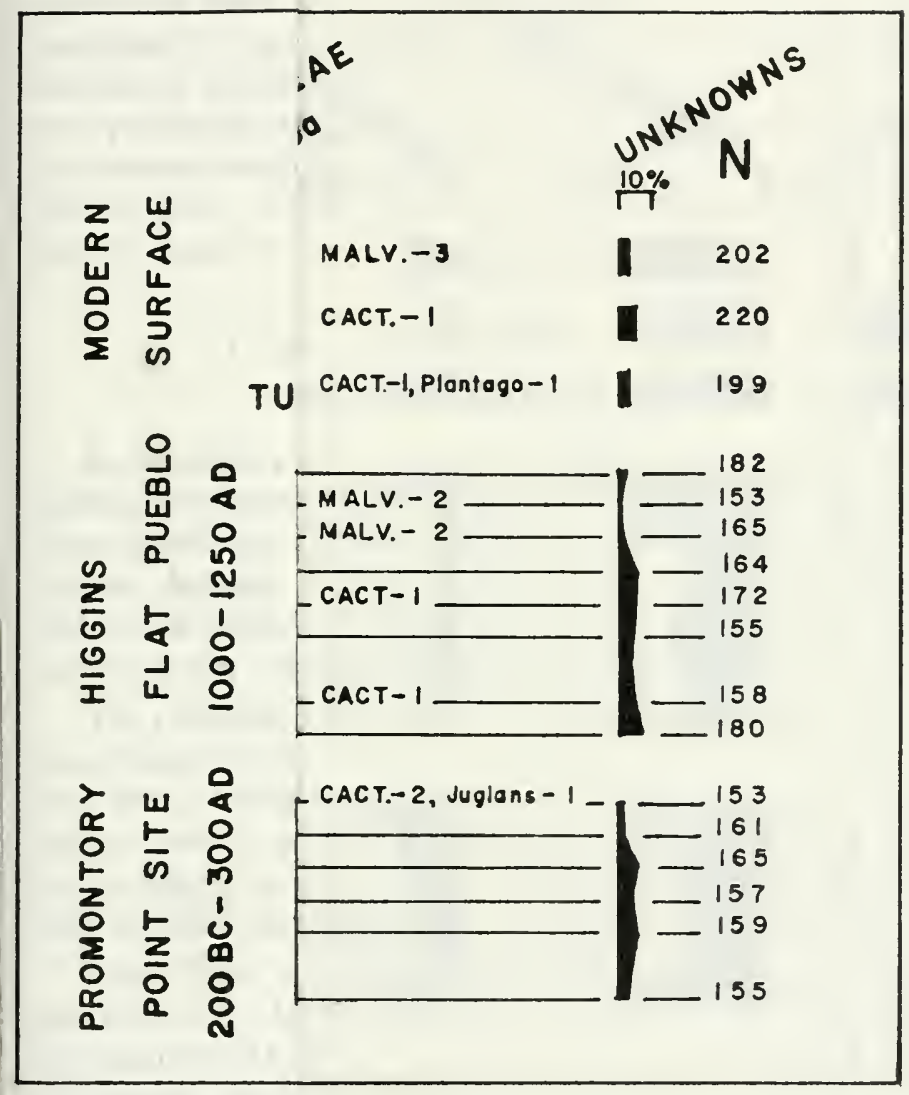

I, New Mexico, area. 

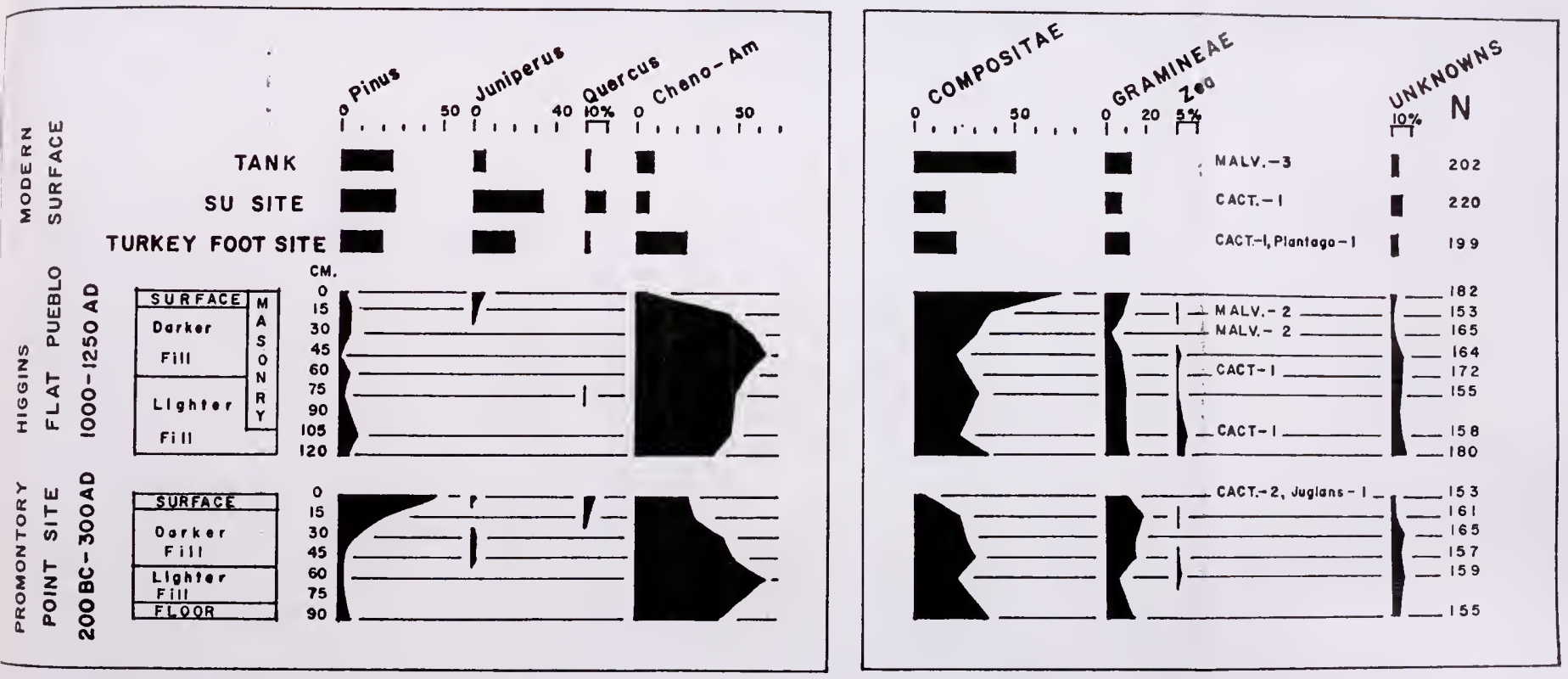

Fic. 76. Analyses of three samples of pollen from modern surface and pollen diagrams of two archaeological sites from the Pine Lawn, New Mexico, area. 
results of the pollen counts of three surface samples as well as the pollen diagrams from two archacological sites in the Pine Lawn, New Mexico, area. Figures 77 and 78 are graphic arrangements of selected information. Figure 77 shows selected data from the analyses of the best dated samples from archaeological localities in the Vernon, Arizona, area, arranged chronologically according to time estimates provided by the archaeologists. The samples are from the floors of dwelling units or from well-dated occupation levels and so are definitely from the period to which they are attributed. It must be emphasized that the dating in most cases is not absolute; it is only a reasonable estimate. Figure 78 shows certain significant palynological features from the analysis of sediment samples from the modern surface at archaeological and cattle tank localities in the Vernon area. These samples were thoroughly investigated, but only selected data are shown in the text figure.

\section{COMMON NAMES OF POLLEN TYPES AND ECOLOGICAL NOTES}

In this report three main structural categories are utilized when discussing conditions of plant ecology: grasslands, parklands, and forest. Short grasslands (Nichol, 1952, pp. 203-205) are today evident in the Vernon, Arizona, area above 1800 meters elevation. In this zone funiperus and Pinus edulis (pinyon) occur sporadically in favorable microenvironments, but arboreal vegetation is predominantly absent.

The parkland begins (ca. 2050 meters elevation) where Pinus and funiperus become common, but the trees are relatively low and the canopy is very open. At higher elevations (above 2120 meters) the forest zone exists where arboreal vegetation is dominant; $P$. edulis and Juniperus give way to $P$. ponderosa and the canopy becomes more closed. Deep shade is found only at higher elevations than the localities sampled in this study.

Three other categorizations of plants are also used: hygric, arboreal and economic. Hygric plants are those which only grow in a very moist environment (Dansereau, 1957, p. 206). In this report, only Typha and Cyperaceae are included in this group. Salix, Juglans and Alnus might also have been included, but since their water requirements are not so limited they are placed with the arboreal plants.

Arboreal plants are those commonly recognized as trees. In this report the category includes Pinus, Juniperus, Quercus, Salix, Juglans and Alnus.

Economic plants are those which are either cultigens or wild plants considered economically important to man. This group includes Zea, 
Cucurbita and Cleome. Cleome is included because pollen attributable to Cleome serrulata has been found in very high percentages in sediments taken from Pueblo III rooms at Wetherill Mesa (Schoenwetter, 1960b) and Chaco Canyon (Martin and Schoenwetter, Manuscript b) as well as alluvial sediments presumed to date from Pueblo III at Binne-Ettini Canyon (Martin and Schoenwetter, Manuscript a). It is assumed that high percentages of this zoogamous pollen type in cultural contexts are the result of its selection by man for some economic purpose. Today the plant is used by Indian groups in the Southwest as a pot herb, a subsistence food, and a source of pigment. It is usually gathered or allowed to grow as a tolerated weed in agricultural fields (Robbins, el al., 1916; Whiting, 1939).

Since many may be unfamiliar with the scientific names of the plants for which pollen types have been identified in this study, the following list of common names is included. Notes on the ecological contexts in which these plants are usually found are added to facilitate interpretation.

Alnus: Alder. A shrub or low tree common to the flood plains of permanent streams in the upper parkland and forest zones.

Cactaceae: All plants in the cactus family. Most of the pollen found is probably referable to the prickly pear group (Platyopuntia). In the study area these plants typify arid and semi-arid micro-environments.

Cheno-am: Pollen types referable to the Chenopodiaceae (goosefoot family) and the genus Amaranthus (pigweeds). This is an artificial pollen category necessary because the pollen of the two plant groups cannot be presently distinguished with greater accuracy. The designation "cheno-am" follows the precedent of Martin, Schoenwetter and Arms (1961). A common member of the Chenopodiaceae is Chenopodium album (lamb's quarters) and a common member of the genus Amaranthus is Amaranthus palmeri (pigweed). These plants are typical of disturbed sediment conditions such as occur today in arroyos, along roadsides, and along the dissected flood plains in the grassland zone.

Cleome: Probably Rocky Mountain bee weed, Cleome serrulata. Identification of this pollen type is not positive because the pollen grain is very small. The plant today is found in the parkland and grassland zones and is known to be gathered and semicultivated by Indian groups.

Compositae: All plants in the sunflower family. Smaller divisions of this group can be made on palynological grounds but with the equipment available these would have been uncertain. Plants of this family occur under an extremely wide range of environmental conditions.

Cucurbita: Squash. Though the cultivated and wild species of this genus are not separable on the basis of pollen type the context in which the pollen was found implies that this is the cultivated form.

Cyperaceae: All plants in the sedge family. Typically, sedges are found under conditions of hygric environment such as the margins of ponds, marshes, cienagas, ctc.

Ephedra: Mormon tea. Though this is not a very common plant in the area it is occasionally found in the parkland and grassland zones. 


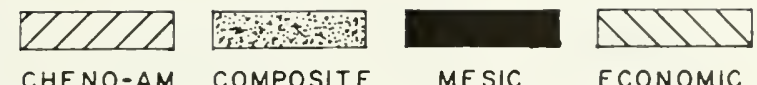

ARBOREAL CHENO-AM COMPOSITE MESIC ECONOMIC

AGE SITE

A.D.

1350 Table

Rock

Pueblo Rm.X

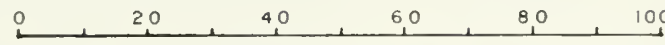

N

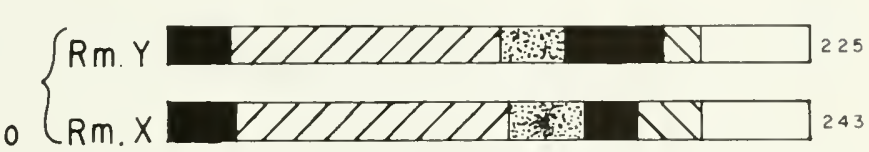

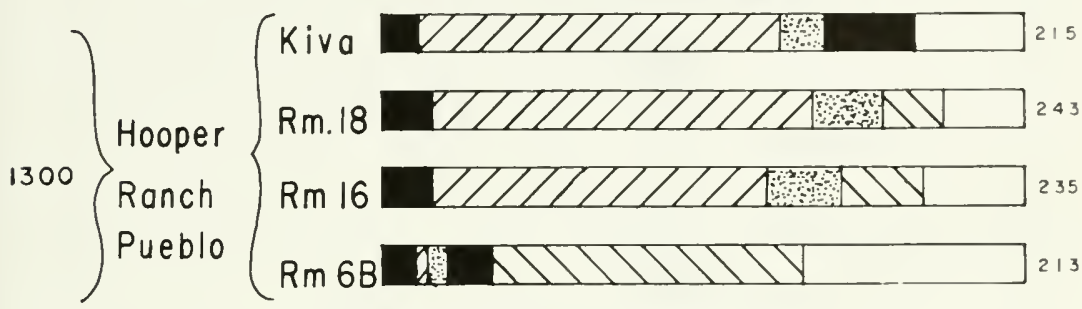

1100 Rim Valley Pueblo $\quad$ L/Z/Z

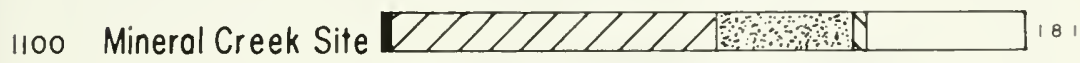

900

$L S-28$

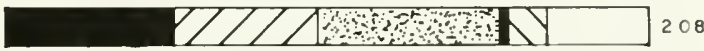

800 Site 30 D/2/7/4 158

$700 \quad$ LS -4

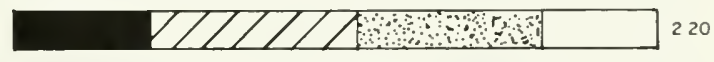

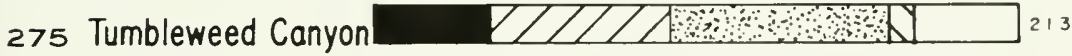
B.C.

1550 Laguna Salada

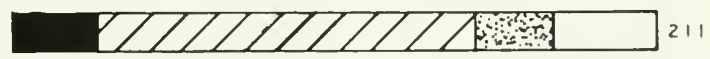

? Little Ortega

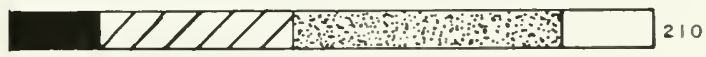

FIG. 77. Important palynological features of samples of pollen from occupation levels at archaeological sites in the Vernon, Arizona, area.

Erratum: For Mesic read Hygric. 
Elev.

in

Meters

1860 Cattle Tank No.

1860 Table Rock Pueblo

1885

LS - 4

1990 LS-34 Pithouse I

2006 LS-34 Pithouse 2

2100 Cattle Tonk No.3

2100

$L S-28$

2240 Rim Valley Pueblo

2250 Hooper Ranch Kiva

2250

Room 18

2250

Room 16

2255 Mineral Creek

2300 Site 30-31 Arroyo

2300

Site -30

QZ7ZA WHA

COMPOSITAE OTHER
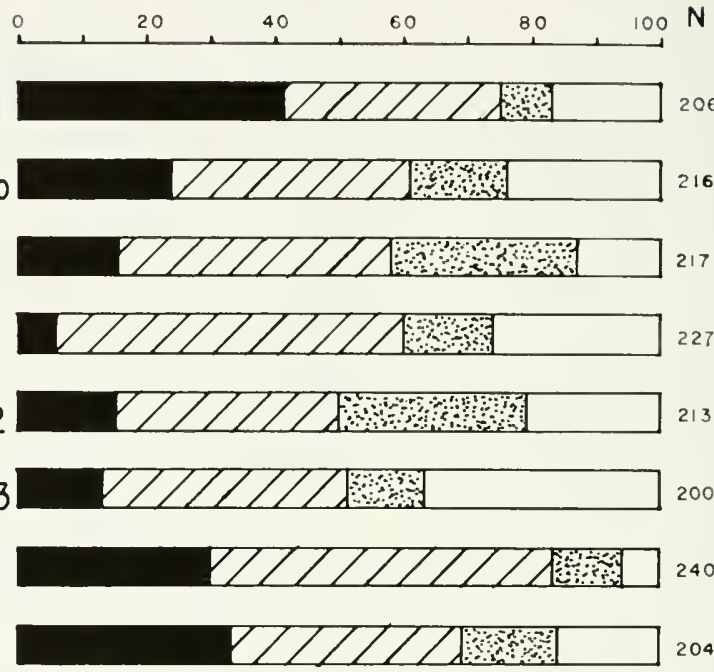

204

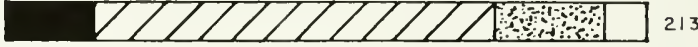

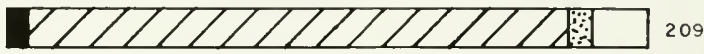
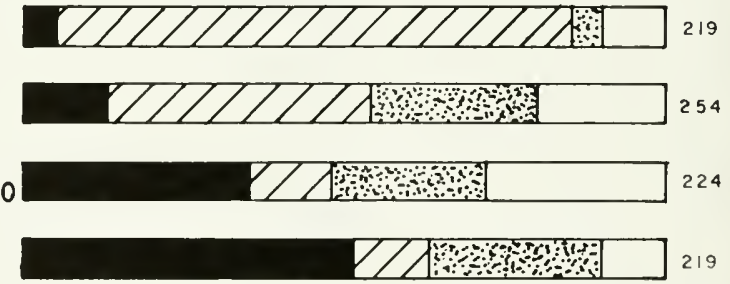
2300 Catfle Tonk No.4

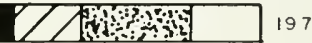

Fic. 78. Samples of pollen from modern surface at various elevations in the Vernon, Arizona, area. 
Gramineae: All plants in the grass family with the exception of Zea. Grasses occur under a wide range of environmental conditions.

Juglans: Walnut. Found today along the flood plains of permanent streams below the parkland border.

Juniperus: Juniper, locally called cedar. Typically, this is a plant of the parkland and lower forest zones, but it may extend onto the grasslands.

Malvaceae: All plants in the mallow family. Most of the pollen grains are probably referable to the genus Sphaeralcea (globe inallow). Pollen of the genus Gosspium (cotton), which belongs in this family, was not observed though its pollen is distinctive. Sphaeralcea grows under a variety of environmental conditions.

Pinus: Pine. Smaller groupings than those on the generic level can be made on the basis of pollen statistics (Martin, Schoenwetter and Arms, 1961) but the data and the equipment did not lend themselves to this type of analysis. Pines are primarily parkland and forest plants, but a few may invade the grassland zone.

Quercus: Oak; typically found under forest and upper parkland conditions in this arca.

Salix: Willow. Pollen identification is not positive in all instances. Typically, this plant is found along shallowly dissected flood plains in the lower parkland and grassland zones.

Typha: Cattail; a plant of hygric conditions, like Cyperaceae.

¿ea: Corn; exclusively a cultivated plant.

\section{RESULTS}

\section{Arroyo Sites}

Series of sediment samples were collected and processed from five arroyo localities. One series, that from Wet Leggett arroyo in the Pine Lawn area, is directly relevant to this paper since this area was the locale of a group of archaeological finds which are considered of Cochise cultural affinity (Martin. Rinaldo and Anters, 1949). The description of the allurial sediments by Anters allowed placement in time of certain strata, but because of the intervening years of active erosion at Wet Leggett arroyo Rinaldo and I had some difficulty in relocating the stratigraphic sections described by Anters.

It was thought that the samples collected from the strata which contained Cochise artifacts would be the most ancient in the prospective pollen chronology. Unfortunately, none of the 15 sediment samples collected produced enough pollen for analysis.

None of the other series of samples taken from arroyo sites were directly related to the archaeology of the Vernon area, where they were collected. Little work was done on them, since the archaeological samples were mostly productive and a pollen chronology could be recovered. All of the samples were processed, however, and some will be used in the future as part of another report on pollen studies in the Mogollon area. 


\section{Beach Sites}

Two ancient beaches on the shores of playas in the Vernon area have yielded artifacts and other evidence of human occupation (Martin and Rinaldo, 1960a). At the Laguna Salada Site charcoal was recovered which was radiocarbon dated at $1420 \pm 60$ B.C. (Gro 1614). The Little Ortega Site, on a nearby playa, could not be dated directly but the artifact assemblage contained more chipped and fewer ground stone implements than were observed at Laguna Salada, suggesting an older occupation if this culture developed in a manner similar to that of the culture found in Ventana Cave (Haury, 1950, pp. 543-544).

The occupational debris and the artifacts are embedded in the compacted lake silts. The sedimentary types are uniform, no stratigraphy is apparent in the walls of the trenches, and artifacts and charcoal beds occur from the surface to a depth of $75 \mathrm{~cm}$. It is highly unlikely that the cultural materials were washed into their present position or that they were lowered from some upper level by deflation. The sedimentary deposit, and the pollen it contains. appear to date in each case from the period of cultural activity.

The pollen spectra of the Little Ortega Site are distinct from those of the Laguna Salada Site (fig. 75). At Little Ortega, pollen from Compositae is the dominant microfossil throughout all samples, averaging 38 per cent, and Juniperus pollen frequencies are consistently higher than those of Pinus. At the Laguna Salada Site cheno-am pollen is the dominant microfossil throughout all samples, averaging 53 per cent, and Pinus pollen frequencies are consistently higher than those of funiperus. The difference in the pollen spectra cannot be attributed to differences in the local environment, since both sites are situated in approximately the same environment; both are on ancient beaches at approximately the same elevation.

It seems certain that the differences in the pollen spectra reflect environmental conditions at different periods of time: that at the Laguma Salada Site at approximately 1420 B.C. and that at the Little Ortega Site at some other time. The artifacts collected appear to indicate that the Little Ortega Site is earlier in time, and investigation of later sites in the Vernon area reveals no palynological evidence contradictory to this view.

\section{Pithouse Village Sites}

The oldest pithouse sampled was the Tumbleweed Canyon Site in the Vernon area. This site contained charcoal from which a radiocarbon date of A.D. $360 \pm 50$ has been obtained by the Groningen Laboratory. 
The pollen spectrum of the sediment sample taken from the floor of the pithouse is unlike those found at the earlier beach sites (figs. 75 and 77). There is about as much cheno-am pollen (27 per cent) as Compositae pollen ( 34 per cent) and about 18 per cent of arboreal pollen. Nine pollen grains of Zea were recovered.

Two samples from the pithouse fill show results dissimilar to those of the floor sample. The sample at $15 \mathrm{~cm}$. depth has a higher percentage of cheno-am pollen, but otherwise there is no essential change except that Zea pollen is absent. The sample at $5 \mathrm{~cm}$. depth is dominated by pollen of Pinus and, in fact, is similar to no other subsurface sample analyzed. A surface sample was, unfortunately, not collected from this site. At present, there are no pine trees in the immediate vicinity.

The Promontory Site (Martin, Rinaldo and Antevs, 1949) is located in the Pine Lawn area and is a pithouse village tentatively dated between 300 B.C. and A.D. 500 by archaeological estimate. It is located on a ridge in an area of pine forest with some juniper and oak at 6500 feet elevation. The sample from the floor (fig. 76) has 42 per cent cheno-am pollen and nearly as much (37 per cent) pollen from Compositae. The percentage of Pinus pollen is surprisingly low, considering the present flora of the site area. No Zea pollen was found in the analysis of the floor sample.

In the samples from the pithouse fill gradual changes in the pollen percentages can be observed through time. Cheno-am pollen reaches its maximum frequency at $60 \mathrm{~cm}$. depth $(30 \mathrm{~cm}$. above the floor) and then begins a continuous decline. Zea pollen is observed at the 60, 45 and $15 \mathrm{~cm}$. levels. At the $45 \mathrm{~cm}$. level Juniperus pollen is first seen; Quercus makes its appearance at the $15 \mathrm{~cm}$. level. From the $45 \mathrm{~cm}$. level to the modern surface, the percentage of Pinus pollen increases consistently until at the surface it dominates the pollen spectrum. These changes are evidence of a gradual but persistent change in local flora from the time of the construction of the pithouse. The present forest conditions observable and demonstrated in the pollen spectrum of the surface sample were apparently not to be seen at the time the pithouse village was occupied. At that time there must have been fewer trees, but cultural factors such as land clearance or biological factors such as blight have equal priority with environmental change in accounting for the phenomenon.

The SU Site (Martin and Rinaldo, 1940), which dates from the same period as the Promontory Site, was also tested. Only the surface sample produced sufficient pollen for analysis (fig. 76).

Site LS-34 in the Vernon area consists of two pithouse areas, both of which were sampled, and dates some time earlier than A.D. 700, since only plain brown ware was found. Only the sample from the surface 
could be analyzed from Pithouse 1. At Pithouse 2 (fig. 75) the sample from the floor did not yield to analysis; the fill and surface samples which could be analyzed are dominated by cheno-am pollen and may date from a period later than the floor sample by any number of years. Three pollen grains of zea were found in the surface sample from Pithouse 2.

At Site LS-4 in the Vernon area, no floor was observed in a pit going down to $90 \mathrm{~cm}$., but field notes indicate that it may have been missed. Since the analysis of samples from the 60 and $90 \mathrm{~cm}$. levels yielded similar results (fig. 75), it is assumed that they are representative of environmental conditions in existence at the time the pithouse was constructed. Pottery recovered indicates occupation about A.D. 700 or 800 .

The sample from the lowermost level of Site LS-4 shows a near equivalence of cheno-am and Compositae pollen percentages (32 and 29 per cent), and arboreal pollen frequencies of about 20 per cent (figs. 75 and 77). At the $60 \mathrm{~cm}$. level the percentage of Pinus pollen increases 5 per cent at the expense of Compositae pollen, but this is not statistically significant.

Site 30 (Martin and Rinaldo, 1960a) is a pithouse village located in pinyon-juniper parkland at 7000 feet elevation in the Vernon area. During the excavation of a trench in one house, a sub-floor pit was discovered (fig. 75). The sample from the pithouse floor $(110 \mathrm{~cm}$.) did not yield to analysis, but it is assumed that the sub-floor pit was constructed during the period of major occupancy and that the pollen in the sediment sample taken from the pit approximates that which would have been recovered from the floor sample. On the basis of radiocarbon dating and pottery typology the period of occupation is estimated at A.D. 600 to 800 , probably a little closer to A.D. 800 (Martin and Rinaldo, 1960a, p. 120).

As with the other pithouse sites, the cheno-am pollen frequency (29 per cent) approximates that of the Compositae pollen frequency (39 per cent) in the lowermost sample (figs. 75, 77). The arboreal pollen frequency again is nearly 20 per cent. In the upper levels of the fill, Compositae pollen dominates the pollen diagram until the surface sample is reached, when arboreal pollen shows greater frequency. The gradual rise in Pinus and Funiperus pollen percentages from the $40 \mathrm{~cm}$. level to the surface is similar to that observed at the Promontory Site.

Samples were also collected at Turkey Foot Ridge in the Pine Lawn area (Martin and Rinaldo, 1950a), but they proved unproductive except for the surface sample (fig. 76).

One sample was collected and processed from LS-50, a pithouse site in the Vernon area tentatively dated A.D. 800 on the basis of pottery. 
Since no room fill or floor was discovered in the test pit the single sample was not analyzed.

\section{Pueblo Sites}

From the pottery found at the surface of Site LS-28 in the Vernon area, this pueblo appears to have considerable occupational depth. Sherds from the fill of a test pit dug into one room for purposes of sediment sample collection did not aid in dating the room more precisely than the pueblo itself; an estimated date between A.D. 900 and 1250 may be given to the floor sample analyzed.

The floor sample from LS-28 gives palynological results similar to those of the pithouse sites: cheno-am and Compositae pollen percentages are sub-cquivalent and arboreal pollen is about 20 per cent. Pollen of economic plants occurs as well as some pollen of hygric plants (figs. 75 , 77). In the upper levels, cheno-am pollen dominates the pollen diagram (fig. 75).

The South Leggett Site in the Pine Lawn area (Martin and Rinaldo, 1950b), a pueblo dating A.D. $900-1050$ by archaeological estimate, was sampled but only the surface sample produced significant results (fig. 76).

Higgins Flat Pueblo is located near the San Francisco River in the Pine Lawn area. The ruin is dated between A.D. 1000 and 1250 and is recognized as having been constructed in three stages (Martin, et al., 1956). In excavating a portion of one room for purposes of collecting samples it was found that the fill extended beneath the masonry walls (fig. 76). As the room excavated was not one of those included in the first stage of construction at this site it appears that the masonry was laidd down upon a sediment surface which was part of the general dwelling area before walls were put up to enclose it. Thus the sample collected at $120 \mathrm{~cm}$. (fig. 76) represents an early period in the history of occupation of this site, and this sample more probably dates about A.D. 1000 than about A.D. 1250.

The pollen diagram from Higgins Flat Pueblo (fig. 76) shows a basic similarity to that from Site LS-28 in the Vernon area (fig. 75). In both instances the lowermost sample yields sub-equivalent percentages of chenoam and Compositae pollen and some Zea. The amount of arboreal pollen in the oldest sample from Higgins Flat Pucblo (4 per cent) secms uncharacteristic, but it will be noted that the lower levels at the Promontory Site also contained unusually low arboreal pollen percentages, and these two sites are in the same arca. With the exception of the two uppermost levels, the samples taken above the datable level are dominated by chenoam pollen, as is the case at LS- 28 . 
The Mineral Creek Site in the Vernon area is dated by archaeological estimate on the basis of pottery types and constructional features at about A.D. 1000-1200 (Martin, Rinaldo and Longacre, 1961, p. 165). It is unfortunate that a tree-ring date cannot be given for this ruin so as to fix its placement in time with more precision, since pollen frequencies in the floor sample from one of the rooms are definitely dissimilar from those obtained from floor samples in the earlier pithouse and beach sites. The probable range of error on the date is less than 200 years. At a maximum, the change in the pollen record could have occurred no earlier than A.D. 900 and no later than A.D. 1300. As the change is also in evidence in the $105 \mathrm{~cm}$. sample from Higgins Flat Pueblo, it may have taken place after A.D. 1000.

In the floor sample (figs. 75 and 77) cheno-am pollen forms the major portion of that observed (51 per cent), and the dominance of cheno-am pollen frequencies continues in the subsequent samples. This contrasts strongly with the floor samples from all earlier dwelling units, where cheno-am and Compositae pollen frequencies were sub-equivalent. Arboreal pollen accounts for only 1 per cent of the floor sample, pollen of economic plants for another 2 per cent. Pollen of hygric plants also occurs.

Rim Valley Pueblo is located at 6800 feet elevation in the Vernon area and dates A.D. 1000-1200 by estimates based on the pottery types recovered. The floor sample and the upper samples are dominated by cheno-am pollen (fig. 75). The floor sample yields significantly higher percentages of arboreal pollen (22 per cent) than the floor samples from other pueblo sites (figs. 75 and 77), but the elevation may be a complicating factor here. Pollen of economic plants was observed in only one of the levels of room fill, and it was Cleome pollen rather than Zea or Cucurbita.

Hooper Ranch Pueblo in the Vernon area (Martin, Rinaldo and Longacre, 1961) was the archaeological site most intensively sampled. The site is located on a small rise near the Little Colorado River, a few yards from a large grove of walnut trees. One of the reasons for sampling this site intensively is the fact that clearly defined stages are seen in the constructional development of the pueblo. In the first stage a small pueblo was built and occupied for an unknown length of time. Later, the pueblo was filled with trash and a second pueblo was built on top of it. At some time a Great Kiva was constructed adjoining the pueblo. It was hoped that significantly distinctive palynological features could be demonstrated for the two stages of construction and that environmental changes could be recognized which would account for the building activity and/or the abandonment of the site. On the basis of pottery types 
it is estimated that the period of occupation was between A.D. 1200 and 1375 (Martin, Rinaldo and Longacre, 1961, p. 167).

Room 6B (fig. 75) dates from the early period of construction. Floor II, at a depth of 2.3 meters below the present surface, was sampled as representative of the oldest sediment attributable to the site. The fill from 2.05 to 2.25 meters was finer-grained and of a darker color than that above it and is considered occupation fill, while that from 0.9 to 2.05 meters was lighter and coarser-grained and is considered as trash fill. At $90 \mathrm{~cm}$., Floor I was laid down during the later stage of construction.

The samples from Floor II and the occupation fill in Room 6B yielded higher percentages of Zea pollen than any others collected in the course of this project and are the only ones in which Cucurbita pollen was observed. Typha pollen occurs in relative abundanec, and the percentage of arboreal pollen is low. Because of the high proportion of pollen of cultigens, the pollen types which reflect the regional environment (chenoam, Compositae, arboreal) are necessarily in low proportion and the sample from Floor II appears anomalous in figure 77.

As the samples progress upward through the occupation and trash fill in Room 6B, it is seen that the proportion of pollen of cultigens steadily decreases and that of cheno-am pollen steadily increases; thus, the sample from Floor I shows a dominance of cheno-am pollen and almost no pollen of cultigens, no pollen of Typha or Cyperaceae, and a larger proportion of arboreal pollen than Floor II.

Rooms 16 and 18 belong to the later period of construction. The former was sampled stratigraphically; the latter, except for the floor and surface samples, was sampled in a random manner. Room 16 overlies Room 6B; Room 18 lies near the edge of the ruin closest to the grove of walnut.

In both rooms, Floor I contains small percentages of pollen of cultigens, a dominance of cheno-am pollen, no pollen of hygric plants and about 5 per cent of arboreal pollen (figs. 75, 77). In short, analysis of samples from Floor $\mathrm{I}$ in all three rooms is essentially equivalent. Samples from the upper levels in Rooms 16 and 18 (fig. 75) show no significant increase in Zea pollen, though significant fluctuations can be observed in the amount of Cleome pollen. Typha and Cyperaceae pollen grains occur sporadically and the proportion of arboreal pollen fluctuates from level to level and room to room; it also undergoes internal fluctuation from dominance by one genus to another at various levels.

Samples from the Great Kiva were collected stratigraphically. The floor sample from the kiva shows a far greater resemblance to the sam- 
ples from Floor I in Room 16 and Room 18 than the sample from Floor II in Room 6B, and the samples from the kiva fill are more similar to those taken from fill in the upper rooms than to those taken from the occupation fill in Room 6B. Apparently, the kiva was built during the second constructional period. Seriation studies on the pottery contained in the kiva may substantiate this conclusion.

Are the construction changes that have been observed at Hooper Ranch Pueblo correlative with environmental changes? This basic question is not completely answered by the data obtained by palynological analysis. Certainly, there are differences in the pollen spectra recoverable from the earlier and later construction periods, but since the basic change is in the percentage and types of pollen of cultigens it must be recognized that the difference could be accounted for on cultural grounds. The Zea pollen may be present in larger quantities because the cornfields were closer to the pueblo at that time; or perhaps people were bringing ripe corn tassels into their dwelling; or possibly more corn was being grown.

A certain coincidence of palynological features argues for the occurrence of an environmental change. The percentage of Cleome pollen increases in the later period of construction; pollen of hygric plants occurs more consistently in the earlier period and more sporadically in the later period; pollen of Juglans occurs consistently in the later period and rarely in the earlier period: and arboreal pollen is more important in the later period. If the change in the pollen record were due to the cultural changes noted above, it might be expected that the percentage of all other pollen types would increase as the percentage of zea pollen decreased. The percentage of Typha pollen, however, decreases in the later period.

Whether the cause is largely cultural or largely environmental, some shift in economic emphasis seems apparent at Hooper Ranch Pueblo. If the dating of the Great Kiva to the later construction period is correct, a shift in religious emphasis may also be indicated. It is tempting to speculate that as the corn harvest became less dependable the inhabitants of the site turned to religion in an attempt to restore the previous favorable conditions.

Table Rock Pueblo, in the Vernon area, is also situated near the Little Colorado, but it is located on a rocky hill overlooking a broad stretch of the river (Martin and Rinaldo, 1960b). It is the youngest site sampled, dated by radiocarbon (A.D. $1345 \pm 50$ ) and dendrochronology (outermost ring A.D. 1331) at about A.D. 1350. Soon after the site was built the upper Little Colorado drainage basin area was abandoned by 
pueblo-building peoples. It was hoped that pollen analysis at this site would give some clues to the reasons for abandonment.

Two rooms were sampled stratigraphically. As these rooms had not been previously excavated or numbered, they were designated Room X and Room Y. In reference to the site map shown in Martin and Rinaldo (1960b, p. 164), Room X lies east of Room 25 and Room Y' lies east of Room 1.

Pollen spectra from the floors and fills of both rooms are nearly identical (fig. 75). The samples are dominated by cheno-am pollen; they are unusually rich in pollen of Ephedra; they have a relatively high proportion of pollen of Typha and Cyperaceae; they have a low proportion of pollen of economic plants; and they have arboreal pollen percentages significantly higher than those of Floor II at the Hooper Ranch Pueblo (Room 6B) but not significantly different from those of Floor I (Rooms 16 and 18). Except for the Ephedra and the indicators of hygric environment, the pollen diagram is quite similar to that of the later construction period at Hooper Ranch Pueblo.

Site LS-24, a pueblo in the Vernon area dating from the same period as Table Rock Pueblo, was sampled but not analyzed, as no definite floor was established in the test pit.

\section{Surface Samples}

Surface samples were collected at the archaeological and arroyo sites as controls on underlying samples. A few surface samples were also collected from cattle tanks, where open water surfaces make conveniently sampled pollen traps (Martin, Schoenwetter and Arms, 1961).

It is assumed, unless evidence has been found to the contrary, that the surface samples are representative of the pollen that is being disseminated today in the area of collection. On this basis, the surface samples from Pithouse 2 at LS-34 and Table Rock Pueblo (fig. 75) have been discarded from consideration. In these two instances, pollen of economic plants which was found at the surface may represent contamination from lower occupation levels.

The analysis of surface sediment samples in the Vernon area is shown (fig. 78) in terms of three major palynological features (arboreal, cheno-am and Compositae pollen) plotted against elevation. The surface samples from the Pine Lawn area (upper part of fig. 76) were collected at 2050 meters (cattle tank), 1975 meters (Higgins Flat Pueblo), 2100 meters (SU Site and Turkey Foot Ridge), and 2135 meters (Promontory Site).

Contrasts may be recognized in the results of surface samples taken in the two areas. In the Pine Lawn area, surface samples taken at eleva- 
tions greater than 2100 meters are dominated by arboreal pollen; in the Vernon area, arboreal pollen is dominant only at 2300 meters. In the Pine Lawn area, those pollen spectra which are not dominated by arboreal pollen are dominated by Compositae pollen; in the Vernon area, those spectra which are not dominated by arboreal pollen are dominated by cheno-am pollen or have sub-equivalent frequencies of cheno-an and Compositae pollen.

Generally, the surface samples fall into four categories:

1. Those in which arboreal pollen is the dominant feature; namely, Site 30, Cattle Tank No. 4 and the Promontory Site. These localities are mostly within the forest border. Cattle Tank No. 1 is an exception; it has arboreal pollen as the dominant feature but is located in the grasslands where no trees are visible for some miles.

2. Those in which cheno-am pollen is the dominant feature; namely, those from Hooper Ranch Pueblo and the Site 30-31 arroyo. Surface samples in which cheno-am pollen is dominant are mostly near streams or arroyos.

3. Those in which the cheno-am pollen frequency and Compositae pollen frequency are sub-equivalent; namely, the Mineral Creek site.

4. Those in which Compositae pollen is dominant; namely, the cattle tank from the Pine Lawn area and Higgins Flat Pueblo. In the former instance, the locality is at present filled with water during the entire year and is large enough to be used as a mill pond. This cattle tank is located in the upper parkland zone.

Higgins Flat Pueblo is located near the San Francisco River, where the growth of cheno-am pollen producers might be expected to be great. When the site was visited, the annual crops of Chenopodiaceae and Amaranthus had not yet developed to the point where they could be identified; thus, no data are available at present on whether these plants grow at this locality. An explanation may be found to account for the high proportion of Compositae pollen in the surface sample from this site. The site is located on level ground near the river. The buried masonry of the walls impounds moisture and hinders run-off. Thus, ground moisture reserves at this locality are unusually high for shallow-rooted, non-arboreal plants.

Finally, in comparing the sub-surface samples from archacological sites (fig. 77) with those from the modern surface (figs. 76 and 78), a number of striking similarities may be noted. The sample from Little Ortega is more like that from the tank in the Pine Lawn area than any other archaeological sample. The sample from Laguna Salada is similar 
to that from the Great Kiva at Hooper Ranch. The sample from LS-4 is nearly identical with that from the surface at the Mineral Creek Site. The sample from Floor I in Room 18 at Hooper Ranch Pueblo is very like that from the surface at Pithouse 1 at LS-34.

\section{CONCLUSIONS}

In constructing a pollen chronology for the eastern Arizona-western New Mexico area from the information gathered in this study, two basic problems presented themselves. First, it was necessary to determine the date of the deposition of the pollen recovered and analyzed. Second, it was necessary to determine that the pollen spectra attributed to a given time horizon were actually characteristic of that horizon and not merely characteristic of that particular locality.

The most common method of dealing with these problems is the selection of two or more series of superimposed sediment samples. By evaluation of the stratigraphy of the sampled localities it is possible to determine which of the samples are older and thus to obtain a relative chronology. By comparing one series of samples with another, it is possible to determine roughly how local factors of physiography, sedimentation or vegetation may have affected the pollen record. Then some of the samples, or some of the stratigraphic strata which have been sampled, may be dated by radiocarbon, tree-rings or other dating techniques.

This method, however, has certain limitations if the results are to be applied to archaeology. It is not usual to find many cultural horizons in superposition or material remains deposited throughout sedimentary columns sampled for pollen analysis. Also, the dating of such pollen changes as occur in stratigraphic columns often has a greater range of error than the archaeologist would prefer. This is especially true in the arid Southwest. To date a change in the pollen record in this area to between A.D. 1000 and A.D. 1400, for example, would be insufficient for major archaeological interpretation, because many cultural changes can be recognized within this period of time and the pollen change might be related to all, some, or none of the cultural changes.

In this study, precision in dating many of the sediment samples was possible because samples could be taken from the floors of habitation units. If the dwellings could be dated by ceramic content, dendrochronology or radiocarbon, the date of the sediment samples was particularly well established. Samples collected above the floors of the dwellings could be considered younger than the floor sample, but of unknown absolute age, while those taken from the modern soil surface 
could be considered representative of the present period. Building the chronology thus became a matter of fitting the recovered pollen spectra into their known age relationships.

Since the samples of known date formed the basis of the chronology, and since these samples were found superimposed upon one another only in the few instances where constructional stages were in evidence in the dwelling unit, there remained the problem of determining that the pollen spectrum recovered for a given time horizon was characteristic of that time horizon. The resolution of this problem has been approached by two lines of reasoning.

First, there was one large group of samples which were all representative of the same time horizon: the group of 20 ground surface samples which represent the modern pollen rain. Comparison of these samples, since the plant habitats from which they were collected are known, allows some measure of the effect of local factors on pollen deposition. It was found that surface samples taken from forest or upper parkland habitats usually contain high percentages of arboreal pollen (such as at Site 30. the SU Site, or the Promontory Site). Samples collected from locales where water tables are permanently high (such as the cattle tank in the Pine Lawn area) or where there is a relative abundance of ground moisture (such as Higgins Flat Pueblo), contain high percentages of Compositae pollen. Samples collected from locales near streams (such as Hooper Ranch Pueblo) contain high percentages of cheno-am pollen. A few of the samples do not contain high percentages of any particular pollen type; these are usually from locales in the parkland or grassland zones where streams are not close by and local conditions do not indicate a high ground moisture content.

From these data the conclusion is drawn that local conditions of both vegetation and ground moisture affect the pollen rain at any particular site. It is recognized, however, that the two factors are inter-related, since the vegetation is sensitive to the moisture conditions. If the site sampled is in the upper parkland or forest zones arboreal pollen dominates the pollen spectrum, but this zone is the one in which precipitation values are higher and evaporation values decreased because of increased elevation.

If the site sampled is within the grassland or parkland zones, where there are no trees or the tree canopy is quite open, pollen of non-arboreal plants dominates. The dominant type will be Compositae pollen if there is a relative abundance of water locally available; it will be cheno-am pollen if a stream (permanent or intermittent) flows nearby; it may be neither if local conditions are otherwise. 
If, on the present time horizon, the samples taken from a particular type of locality are consistently similar, it might be expected that the same generalization would hold for former time horizons. On the other hand, for two sites of a given time horizon pollen spectra would be expected to be dissimilar if the same type of locality were not present at both. For example, if local conditions of forest and ground moisture were similar at the Tumbleweed Canyon Site and the Promontory Point Site, the pollen spectra of the floor samples at both sites would be expected to be similar, since both are dated between 200 B.C. and A.D. 500 . If local conditions were dissimilar at the two sites, the pollen spectra would be expected to reflect the difference.

It should be possible, if no climatic change has occurred in the area to disrupt ecological relationships, to determine also the particular type of locality from which the sediment sample has been collected through its pollen spectrum. If the sample contains high frequencies of arboreal pollen, it should come from an area where forest or upper parkland conditions are, or were, locally evident. Similarly, it should be possible to determine if the conditions were those now found below the parkland border near streams or in areas of abundant local ground moisture.

The second line of reasoning evolves from inspection of the pollen diagrams from the various archaeological sites. It is seen that in all of the sites which date later than A.D. $1000 \pm 100$, except for Room $6 \mathrm{~B}$ at Hooper Ranch Pueblo, the entire pollen diagram is dominated by chenoam pollen. In sites which date earlier than A.D. $1000 \pm 100$, excluding the beach sites, the pollen diagrams are dominated by cheno-am pollen only above the floor sample. This shift in the pollen record is noted for sites in both the Vernon, Arizona, area and the Pine Lawn, New Mexico, area. From these data it is concluded that a shift in environmental conditions occurred about A.D. 1000 and that it affected the pollen record at almost all of the localities sampled. Thus, there is some basis for considering that shifts in the pollen records at given sites in the east-central Arizonawest-central New Mexico area may reflect regional changes in environment rather than merely changes in local conditions. If the pollen shift can be observed in pollen diagrams from two or more sites the likelihood is increased that the phenomenon involved is a regional change in environmental conditions.

If the reader will grant that the means of dating the pollen spectra are adequate and that the shifts which occur in the pollen record are regional rather than local fluctuations, the following pollen chronology is evidenced by the results of this study: 
Four major periods of clifferent environmental conditions can be recognized in the palynological data recovered from the archaeological sites. These periods are characterized by differences in the frequencies of pollen types, rather than by differences in the types of pollen found.

The most recent environmental period is characterized by clominance of the pollen spectra by high percentages of cheno-an pollen. It is reeognizable at the Mineral Creek Site, Rim Valley Pueblo, Hooper Ranch Pueblo and Table Rock Pueblo in the floor samples; in about 50 per cent of the samples taken from the modern surface; and in certain levels taken above the floor at the Tumbleweed Canyon Site, the Promontory Site, Site LS-34, Site LS-28, and Higgins Flat Pueblo. Since the oldest floor sample to which high cheno-am pollen percentages may be attributed is that from the Mineral Creek Site, it appears that this period began about A.D. $1000 \pm 100$ and has continued to the present day.

The dating of the change that occurs in the pollen record is crucial to proper interpretation of the effect an environmental change may have had upon eulture. The change had not oceurred when the floor sediment at Site LS-28 was deposited, some time between A.D. 900 and 1250. It had also not taken place when the sub-floor sediment was laid down at Higgins Flat Pueblo, which probably was about A.D. 1000. By the time the Mineral Creek Site and Rim Valley Pueblo were constructed, however, the change had taken place. Rim Valley Pueblo and the Mineral Creek Site date between A.D. 1000 and 1200. The change must be dated earlier than 1200, since it was in effect when Hooper Ranch Pueblo was constructed, ca. 1200.

The bulk of evidence seems to indicate that the change took place about A.D. 1000. For the sake of greater precision, I have added a plus or minus figure of 100 years, placing the shift at $1000 \pm 100$.

Within this first period there is sufficient variation in the frequencies of other pollen types than cheno-am to justify the establishment of substages. In the earlier part of the period (1000 $\pm 100-1200)$ such floral elements as Typha, Cyperaceae, Zea and arboreal plants are not of particular importance in the pollen spectra. In the middle part of the period (1200-1350) pollen of Žea, Typha and Cyperaceae becomes an important feature of the pollen spectra. Figure 77 shows the increase in hygric and economic pollen percentages at this horizon. At Hooper Ranch Pueblo, fluctuations in the frequencies of pollen of hygric and economic plants secm to be correlative with cultural changes.

The youngest part of this environmental period (ca. 1350-1960) is rather poorly sampled but can be recognized in samples from upper levels of stratigraphic sections. A gradual and persistent increase in the 
frequency of arboreal pollen may be observed. This is especially marked at Site 30 and at the Promontory Site, which are today located in the upper parkland and forest zones.

The second period is characterized by pollen spectra in which subequivalent percentages of cheno-am and Compositae pollen are observed. The percentage of arboreal pollen in spectra from this period is greater in the Vernon area (18 to 26 per cent) than in the Pine Lawn area (4 to 5 per cent). The period is in evidence in the floor samples from Higgins Flat Pueblo, Site LS-28, Site 30, Site LS-4, the Promontory Site and the Tumbleweed Canyon Site. A radiocarbon date of A.D. $360 \pm 50$ on the Tumbleweed Canyon Site allows the conclusion that this period extends from A.D. $1000 \pm 100$ at least as far back as the fourth century.

It is interesting to note that Zea pollen was recovered from the floor sample of the Tumbleweed Canyon Site. This datum helps to fill in the chronology of agriculture in the Mogollon area, and bolsters inferences based on the conclusion that agriculture was an established feature of early Mogollon culture.

A gap in the chronology exists between the fourth century A.D. and the fifteenth century B.c. The third environmental period is characterized by the dominance of cheno-am pollen and arboreal pollen frequencies of 15 to 30 per cent. The only samples collected for this period are taken from the Laguna Salada Site, which is dated at $1420 \pm 60$ B.C. by radiocarbon. The terminal date and the beginning date for this environmental period are as yet undetermined.

The fourth environmental period is as yet undated. This period is exemplified by the pollen diagram from the Little Ortega Site, and is characterized by a dominance of Compositae pollen and arboreal pollen frequencies of 15 to 30 per cent. The only means of dating the Little Ortega Site, and thereby dating this environmental period, is through comparison of the artifacts found at Little Ortega with those found at Laguna Salada. At Little Ortega more tools of chipped stone have been found and at Laguna Salada more tools of ground stone were recovered. It is evident from the pollen analyses that the Laguna Salada Site and the Little Ortega Site belong in two different environmental periods, but there is little conclusive evidence to prove which is actually the older. Temporarily, at least, the Little Ortega Site may be considered older than 1400 B.C. on the evidence available through artifact typology.

\section{INTERPRETATIONS AND INFERENCES Clmatic Chavge}

Have there been major fluctuations in climate over the three and one-half millennia encompassed by the pollen record from the eastern- 
Arizona-western-New Mexico area? The question is crucial to interpretation of the palynological data, since a basic objective of this study is the investigation of the relationship of ancient cultures to environment in that area.

It has been shown that at different time horizons different frequencies of certain pollen types were being deposited in the sedimentary column, and reasons have been given to justify the assumption that these changes were responses of the natural vegetation to existing environmental conditions. This is an insufficient basis for claiming that changes in climate have occurred, however, for a change in climate involves a transition from one climatic type to another. Through an investigation of the present climate of the area, and through comparison of pollen spectra from the modern surface and the ancient horizons, it can be demonstrated that no change in climate is evident in the pollen record.

Climates are defined on the basis of long-range patterns of meteorological phenomena. The length of time necessary for the establishment of a climate is disputable, and the measurement of the meteorological phenomena is often far from complete, but classifications have been proposed which describe characteristic climatic patterns. While it is impossible to measure the meteorological conditions prevailing at any time in the past, it is recognized that parallels may be found between biological phenomena existing under present, known, climatic conditions and biological phenomena reconstructed for former time periods. It may be inferred that in situations where the biological phenomena of the present approximate those of the past, the climatic conditions also approximate those of the past.

At present, the area discussed in this report lies in a climatic zone classified as steppe (Trewartha, 1954). The meteorological characteristics of this climate are described as those of the transition belt betwcen the desert climate and the more humid climates. In the steppe climatic zone, the potential evaporation from the soil surface and the vegetation cover exceeds the annual average precipitation, as in the desert, but not by so great an amount. Temperatures are rather severe for the latitude, with relatively extreme seasonal temperatures and consequently large annual temperature ranges. Marked daily ranges in temperature are common. Rainfall is meager, variable, and undependable. Average annual precipitation is $10-20$ inches, variability from normal averages 25-30 per cent, and precipitation is seasonal. Steppe climates are situated on the margins of dry settling tropical air masses associated with subtropical high pressure cells; thus, they are encroached upon by rainbearing winds and their associated storms only for short periods. 
The eastern-Arizona-western-New Mexico area is situated on the western margin of a settling air mass. In the summer, when the air mass is farthest north, torrential convectional showers are formed when heated air rises from the ground surface and meets cooler air settling from above. In the winter, when the air mass is farthest south, rain or snow falls from fronts associated with cyclonic storms.

Summer rains contribute the majority of precipitation allowances (Smith, H. V., 1956) but the pelting rain does not soak the ground to any great depth before running off the surface, collecting in flash floods in the arroyos, and rushing downstream. where it cuts and erodes the flood plains of major and minor drainages. Winter rains are slower. soak the ground, and raise the water table, but they account for a much smaller amount of the annual rainfall. Winter rainfall does not constitute a great water resource for plants, since winter rains occur during the scason when most plants are limited in growth by reduced sunlight and air temperatures. The two dry months preceding the growing season cause much of the soil moisture reserve to evaporate. The classification of the eastern-Arizona-western-New Mexico area as steppe climate is only indirectly a matter of long-term averages of temperature and precipitation values. Basically, it involves recognition of the atmospheric physical system which results in the temperature and precipitation values. If the area undergoes climatic change, then, some change in the physical system must occur and not merely a change in average values of temperature and precipitation. In addition, this change must be in effect for a relatively long period of time. For example, the long-term precipitation pattern in this area could change from biseasonal rainfall to rainfall in all seasons. This would be a climatic change regardless of the amount of rainfall received, for it could only take place if the area did not lie on the western edge of a dry, settling air mass associated with a high pressure cell. On the other hand, one can imagine a shift in precipitation pattern from predominantly summer rains with few winter rains to predominantly winter rains with few summer rains. This situation might occur under the present physical system if the winters were a little cooler (thus bringing more frontal activity), if the summers were a little hotter (since moisture condenses more readily from cooler air), or if winters were cooler and summers were hotter.

Such meteorological changes might be in effect for only one year, in which case they would have little effect on the biota of the area. If they were in effect for some decades or centuries they might have a great effect on the biota; yet, since the physical system which determines 
the climate of the region had not been basically disturbed, a climatic change could not be said to have taken place. The temperature changes initiated in this hypothetical model need not constitute a climatic change, since in the steppe climatic zone extreme seasonal temperatures and large annual ranges of temperature are normal.

The position taken in this paper is that long-term fluctuations in meteorological conditions are of at least two types. If the fluctuation is demonstrably due to a change in the basic physical system which is expressed as a climatic type, it constitutes a climatic change. If the fluctuation is due to changes in the relationship of some meteorological phenomena to others, yet the basic physical system involved is unchanged, it constitutes an enrironmental change or environmental shift. Both climatic changes and environmental changes may affect the biota and/or the cultures of an area. In terms of vegetation and the pollen record, it is expected that climatic changes would be much more marked than environmental changes.

When we return to the palynological data with these distinctions in mind, it should be noted that the present climate of the area studied is expressed differentially in the various samples from the modern surface. At certain locales, arboreal pollen is dominant; at others, cheno-am or Compositae pollen is dominant. There is no question that all of the surface samples reflect the same climate, since all come from an area controlled by the same physical system, which is classified as a single climatic type. The differences between them, then, must be recognized as expressions of local conditions, as they operate within the broad framework of the steppe climatic type.

Similarly, it is clear that the palynological characteristics of the ancient sediment samples can be essentially duplicated in certain of the samples from the modern surface. Over the entire time period investigated, the range of variation in the pollen frequencies in the ancient samples does not lie outside of the range of variation seen within samples of the present climate. Thus, no change in climate can be said to be evident in the period of time represented by the ancient samples.

\section{The Nature of Pre-Existing Environmental Conditions}

The demonstration that the climate of the eastern-Arizona-westernNew Mexico area has not undergone change over the past 3500 years proves that the changes in the pollen record are to be relegated to the position of environmental changes or environmental shifts. There remains the problem of determining the nature of these shifts in terms of the 
ecological changes that occurred and the meteorological changes that precipitated them.

Through analysis of the pollen spectra of the modern surface samples and comparison of the plant habitats from which the samples were obtained, it was concluded that local conditions of both vegetation and ground moisture affect the pollen rain at any particular site.

It has been mentioned that high frequencies of arboreal pollen reflect conditions within the upper parkland and forest borders. In terms of meteorological phenomena, this may be due to the lower air temperatures of the higher elevations, to the reduction in evapo-transpiration values that such lower temperatures encourage, or to higher rainfall allowances due to adiabatic cooling of air masses in the highlands.

Surface samples which contain high frequencies of cheno-am pollen have usually been collected near streams. It is known that cheno-am pollen producers are adapted to growth in disturbed sediments and that they germinate and develop only during the summer rainy season. The summer storms which cut and erode the flood plains of streams cause disturbed sediment conditions and create excellent habitats for the producers of cheno-am pollen.

Surface samples in which high frequencies of Compositae pollen occur have been shown to be related to localities where a relative abundance of ground moisture is found. Perhaps more important, these are localities where sediments are not disturbed by erosion and where water tables are relatively high. Along the streams water tables are relatively low, for dissection of the flood plains brings the water table down.

There are no surface samples in which pollen of the hygric plants, Typha and Cyperaceae, occurs. From the habitat preferences of these plants, however, it is evident that where pollen of hygric plants is found conditions of standing water may be inferred. Standing water is a result of poor local drainage. At present, since most streams are actively undergoing erosion in the Southwest, conditions of poor local drainage are rare. There are a few locales where cienagas and ponds are found, however, and these sometimes are fringed by sedges and cattails.

If these characterizations are applied to the environmental periods recognized in the pollen record, the following interpretation of ecological and meteorological conditions may be developed:

Period I-a: A.D. 1350 to the present. The high percentage of cheno-am pollen reflects sediment disturbance occasioned by the present summer rain-flash flood pattern. Some increase in arboreal pollen may indicate a cooling of air temperatures or an increase in effective moisture allowances. 
Period I-b: ca. A.D. 1200-1350. High percentages of cheno-am pollen reflect the sort of summer rainfall and sediment disturbance conditions observable at present. Sporadic and fluctuating percentages of Typha and Cyperaceac pollen, however, reflect local fluctuations in surface water and drainage conditions. During this period standing water must have been more common than it is today. Major fluctuations in percentages of zea pollen appear to be correlative with the fluctuations in surface water and drainage conditions.

Period I-c: ca. A.D. 1000-1200. High percentages of cheno-am pollen reflect meteorological conditions similar to those of the present. The low frequencies of arboreal pollen found at many of the archaeological localities indicate that the sites were located below the parkland border.

Period II: probably before A.D. 350 to ca. A.D. 1000. The subequivalent percentages of cheno-am and Compositae pollen indicate conditions in which sediment disturbance is less pronounced than at present and ground moisture reserves are essentially greater. Higher arboreal percentages are noted in the floors of dwellings from the Vernon area and lower arboreal percentages are noted in the Pine Lawn area. Apparently the sites are located within or just below the parkland border in the former area, and below the parkland border in the latter area. An agricultural economy is evidenced at four out of six sites.

Period II : ca. 1420 B.C. High cheno-am percentages and somewhat high arboreal pollen percentages indicate conditions of sediment disturbance and, possibly, summer flash floods at a locale near the parklandgrassland border.

Period IV: undated. High Compositae pollen percentages and somewhat high arboreal pollen percentages indicate mesic conditions (probably a large pool of water) near the parkland-grassland border.

In the interpretation given above, the problems of ground moisture reserves and conditions which promote sediment disturbance are of crucial importance. Directly involved is the question of arroyo-cutting, for erosion and down-cutting of streams is a major source of sediment disturbance in the Southwest, as well as a means by which water tables may be lowered. As far as such shallow-rooted herbs as the cheno-am and Compositae pollen producers are concerned, lowering of water tables reduces ground moisture reserves.

A widely held hypothesis on the cause of arroyo-cutting is that which has been recently stated by Anters (1955). This hypothesis maintains that the Southwest is a region of intense cyclical drought. During droughts the vegetation cover is reduced, so that when rains do occur there are 
few plants to absorb the water. Rain water, therefore, rushes off the surface, cutting and denuding the flood plains.

Martin, Schoenwetter and Arms (1961) and myself (1960a) have proposed an alternative hypothesis. We feel that only the summer rainstorms contain sufficient energy to cause down-cutting of drainage ways under the present climatic conditions, since winter rains are usually not from high energy storms. We propose that if climatic conditions have remained stable, time horizons in which arroyo-cutting is widespread may be considered periods in which there were more numerous summer storms.

Our hypothesis is primarily based upon palynological information gathered from samples of flood-plain alluvium in southern Arizona. In the stratigraphic sections of alluvium sampled, it was found that high percentages of cheno-am pollen often accompany strata which reflect conditions of arroyo-cutting. Since it is known that the disturbed sediments occasioned by the present summer rain-flash flood pattern are ideal habitats for cheno-am pollen producers, the inference is made that arroyo-cutting and disturbed sediments are associated with heavy summer rainfall. The down-cutting effect would be the same if the storms were more numerous or more torrential. Since summer storms are usually convectional downpours, any storm probably contains more than sufficient energy to initiate and continue erosional activity; thus, an increase in the number of storms seems probable during periods of arroyo-cutting.

In the pollen record from southern Arizona, high percentages of Compositae pollen are found at certain time horizons. These are not explained as due to an increase in the number of winter storms, since it is not known whether the Compositae are as much affected by winter precipitation as the cheno-am pollen producers are by the summer precipitation. High frequencies of Compositae pollen, however, are often found in spectra correlated with soil zones, cienaga soils, and other indications of quiet, steady drainage of the flood plains. This suggests the possibility of higher water tables during periods when Compositae pollen is deposited in high frequencics. I (1960a) have maintained that higher water tables would not have formed without a substantial increase in the amount of winter rainfall to make up the difference in annual precipitation caused by the reduction of summer rainfall.

Table 21 shows the similarity between the results of this study and the results proffered by Martin, Schoenwetter and Arms from southern Arizona. A few points of difference exist, the major one being that Period II is characterized in the study area by a near equivalence of cheno-am 
and Compositae pollen percentages, while the same time period is characterized by a dominance of Compositae pollen on the southern Arizona flood plains. Two explanations might be given to account for this discrepancy: first, the sites in the north are archacological localities rather than flood-plain alluvium and so are not subject to the same immediate ground-water conditions; second, definite distinctions now exist between plant communities in the two areas, so that it is not improbable that such distinctions existed in the past.

Another point of difference involves Period $[-b$, which is missing in the sequence from southern Arizona. As it is recognized that Period I-b involves local fluctuations in drainage, this is not an important difference. Finally, there is the difference in the dating of the shift from Period II to Period I-c. Martin's information allowed an estimate of A.D. 1200 for this transition but is not as sensitive as that from the study area, which calls for definitely earlicr placement.

Of far more importance is the similarity between the pollen spectra from the two areas, especially as concerns fluctuations in the record of cheno-am pollen. If cheno-am pollen is recognized as an indicator of summer rainfall conditions and sediment disturbance, the periods of heavy and hight summer rainfall in the study area and southern Arizona are seen to coincide.

Adopting the hypothesis that cheno-am pollen percentages reflect rainfall conditions, I interpret Periods I and III as periods when a pattern of numerous summer rainstorms contributed the majority of available water to plants in the eastern-Arizona-western-New Mexico area and initiated conditions of sediment disturbance and arroyo-cutting. During periods II and IV summer rainstorms were not so numerous, water tables were higher, dissection of flood plains was not widespread and, possibly, winter rainstorms were more numerous than they are now.

If the similarity between the pollen chronologies is accepted at face value, it appears that Period IV correlates with an extremely ancient environmental period. While this may be true. the possibility exists that the argument of antiquity for Period $\mathrm{IV}^{\prime}$ based on artifact typology is erroneous and that the Little Ortega Site is actually not older than the Iaguna Salada Site. It would be possible, in that case, for the pollen spectrum of the Little Ortega Site to be indicative of environmental conditions in effect during the transition from Period III to Period II.

\section{Relationship of Preitistoric Environients to Preinstory}

Certain major cultural developments are well known in the easternArizona-western-New Mexico area. The first inhabitants were hunting- 


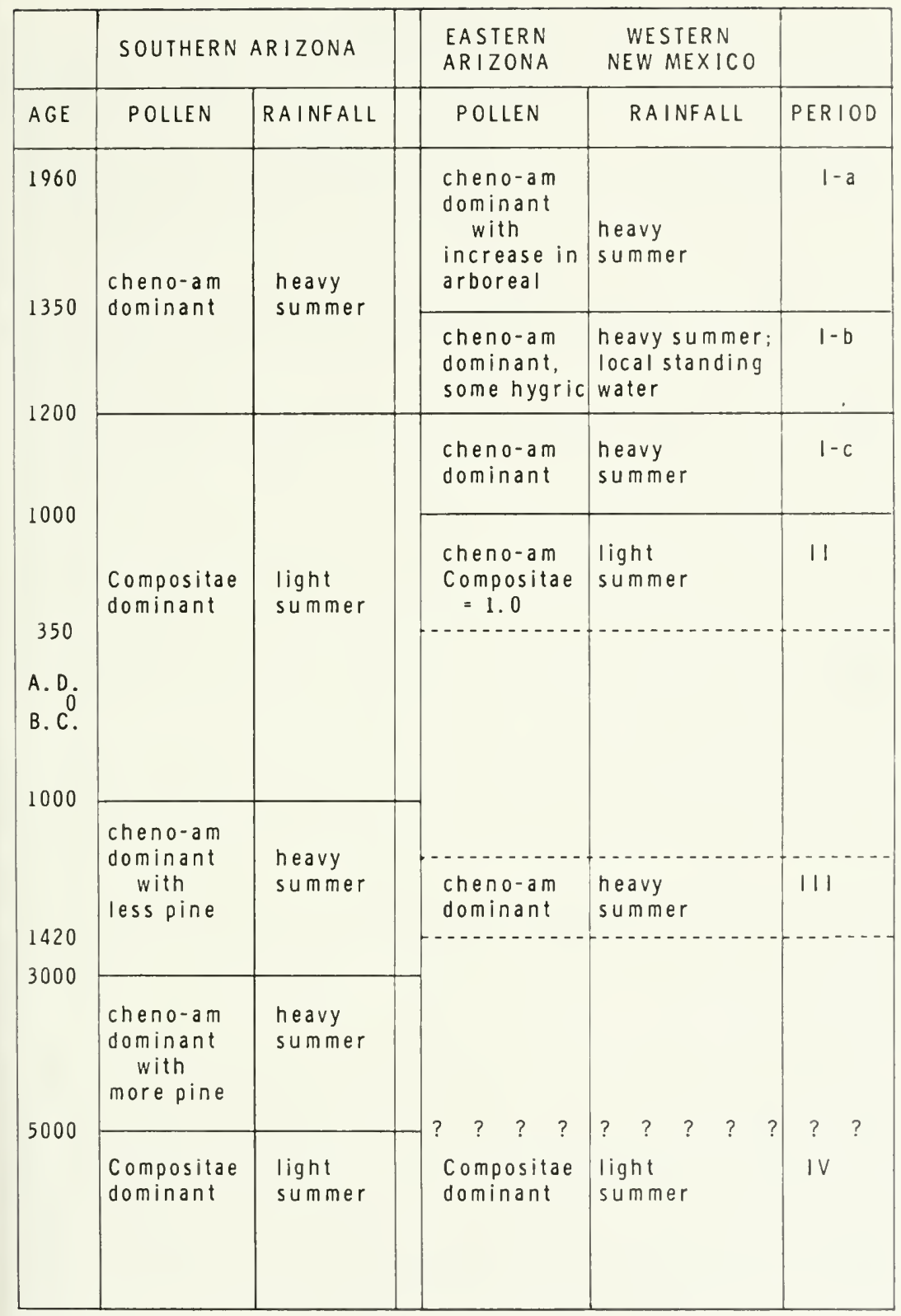

TABLE 21.-COMPARISON OF POLLEN CHRONOLOGIES 
gathering peoples who built no permanent dwelling units, but who may have had some agriculture. At a later time a group of sedentary pithouse village dwellers occupied the region, presumably practicing more agriculture than the early inhabitants. Still later, people in the area began building pueblo dwelling units and were possessed of a more highly developed agricultural culture. It is believed (Martin, Rinaldo and Anters, 1949) that the sequence of development involves a single culture.

In the eastern Arizona section of the area, a recent site survey (Martin, Rinaldo and Longacre, 1961, pp. 147-164) has given much information on settlement patterns through time. The survey indicates that occupation was continuous from about 2000 B.C. to A.D. $1400 \pm 50$. but population size was not always consistent. A steady increase in population is recognized for the period between A.D. 900 through 1300; by 1500 , however, the area had been abandoned by pottery-making, pueblo-building peoples.

In addition, changes are evident through time in the location of sites. Before A.D. 500, sites were located on hills and ridges in the Valley of the Little Colorado River, or on flat areas on the sides of mesas. Between A.D. 500 and 1100, location of sites seems equally divided between the bluffs overlooking the valley, hills on the valley floor, and the river valley floor. Sites which date between 1100 and the time of abandonment, however, are generally located on hills on the valley floor.

The change in environment that occurred between Periods IV' and III seems to have had little effect on the unspecialized cultures of that day. The shift in rainfall pattern and the consequent lowering of water tables and increased dissection of the flood plains could not have had much effect on the water requirements of a nomadic hunting and gathering people. The increased availability of amaranths may have affected their dietary preferences and perhaps stimulated cultural interest in cultigens known in other areas. However, there is no evidence of a change in social habits.

A long gap in chronology separates the cultures of Period III from those of Period II. Whether or not a genetic relationship existed between them, it is evident that the people of Period II had an essentially different relationship to their environment. These people were sedentary and they grew corn. Many of their pithouse villages were located away from the large permanent streams that today drain the area through dissected flood plains. From the pollen record it appears that they lived close to or below the parkland border, though today the sites are often found in the open forest zone. During this period water tables were higher and even minor streams near their villages were probably permanent sources of supply. 
As they were agriculturalists they had taken a major step toward cultural improvement, but as they became more dependent on crop plants for the continuance of their way of life they became more directly limited by the water requirements of their crops. The earlier huntinggathering economy could operate with reasonable efficiency regardless of water sources and resources. The sedentary agriculturalists were far more committed to their environmental resources than the nomads and far more vulnerable to environmental change.

In view of the environmental conditions under which they lived, it seems likely that the pithouse agriculturalists were rain farmers. The major factors affecting the growth of corn are length of growing season, available water for germination of the crop, and available moisture for maintenance of the crop once it has started to grow. The effective crop season in four years out of five in this area is between 120 and 150 days (Baker, 1936) - about the same as that in the corn belt of the Midwest - and is not much of a detrimental factor. Under the present conditions of heavy summer rainfall and low water tables, dry farming is a precarious livelihood in the steppe climatic zone because in many years moisture reserves are insufficient by the end of the frost season to germinate the crop. The summer rains may be sufficient to mature the crop, but the immature plants are often washed out or beaten down.

Under the environmental conditions postulated for Period II, however, winter ground moisture reserves would have been greater at the beginning of the growing season, either because of the greater number of winter rains or the higher water tables or both, and the fewer summer rains would have been less destructive.

Near the end of Period II, the construction of pueblo dwelling units began in the Mogollon area. It is fairly evident that the pueblo dwelling unit was borrowed from the Anasazi, for it has a longer development in the north (Roberts, 1939), and the amount of Anasazi trade pottery and religious ideas very obviously increased in Mogollon culture after A.D. 700 (Jennings and Reed, 1956). No change in environmental conditions can be demonstrated as having occurred simultaneously with the shift to pueblo construction, but it can be recognized that as the change in house form occurred there was a shift in the placement of the sites. In effect, the culture is seen to move from one environmental niche to another. After A.D. 900 more and more sites were located near the major drainage ways, and fewer are found along the bluffs and ridges above the valley floor.

What could have been the advantage of such a move? One advantage would have been that irrigation could be practiced on the valley floor 
much more easily than in the highlands. The practice of irrigation could have been diffused from the Anasazi with the pueblo and other cultural items. Alluvial flood plains along the permanent streams would have been good locales for flood-water irrigation or diversion ditch irrigation during this period, since the streams were not decply dissected.

With irrigation, a rise in population may have occurred consequent upon an increase in the food supply. The communal dwelling unit would have been a practical and effective means of dealing with a larger number of people.

The advent of the conditions of Pcriod I-c, with a heavy summer rainfall pattern similar to that of the present, must have made dry farming an almost impossible practice. Significantly, most sites are located near permanent drainage ways or permanent springs. As there is no existing evidence of large scale irrigation works, the crops may have been irrigated by damming the permanent stream so that its waters flooded the cropland periodically or by short diversion ditches. In Period I-b there is ample evidence of local areas of standing water near the sites. This could be explained very well by the presence of irrigation ditches or dams.

The use of irrigation in the valleys near permanent drainages might have been a cultural advancement, but it could not have lasted long under the environmental conditions postulated for Period I. Sooner or later dissection of the flood plain by high energy summer storms would have caused the water table to fall to the point where irrigation could not be practiced. Since dry farming could not be practiced either, the area would have had to be abandoned if the culture remained committed to cultigens.

If the interpretation of environmental changes set forth above has validity, it should be possible to demonstrate that changes occurred over the entire area of the steppe climatic zone in the Southwest. As yet other pollen studies from this area are unpublished, and though environmental shifts may be evident in the pollen records adequate dating on these shifts is lacking.

One source of confirmation for the interpretation of the environmental shifts presented is the cultural record. Not a few cultures are known to have inhabited the steppe climatic zone of the Southwest during the past 3500 years, and certain cultural changes have been determined. If these cultural changes can be shown to date from the same periods as those in the eastern-Arizona-western-New Mexico area, and if they can be interpreted as due to similar shifts in rainfall pattern, some degree of support for the hypotheses presented above may be gained.

In that part of the area where Anasazi culture was dominant, there are some interesting cultural phenomena which cluster about the dates 
we have determined for the environmental shifts (Jennings and Reed, 1956). A.D. 1000, the approximate date of the transition from Period II to Period I, marks the beginning of an intensive period of areal expansion for Anasazi culture in PII. This period ends about 1200-1250. A.D. 1200 1350, the time span of Period I-b, marks the apogee of Anasazi culture (PIII) but also marks a period of contraction, with Anasazi culture moving back into its nuclear areas and abandoning its new frontiers.

It is known that the Anasazi had irrigation systems at Mesa Verde, but these are not precisely dated (Stewart and Donnelly, 1943). If we assume that the Anasazi had methods of water control during PII, the cultural changes described can be explained on the basis of the environmental shifts postulated.

With the advent of conditions of predominantly summer rainfall about A.D. 1000 more of the steppe zone would have been open to irrigation along permanent drainage ways, since less water would have been retained in the upper reaches of the highland watersheds and the permanent streams would have carried the excess. The Anasazi may have pioneered such newly available territory.

As in the area investigated in this study, erosive action would not have been long in reaching the permanent streams, which would then have become useless. Abandonment would have resulted. Sites in the nuclear areas may also have experienced an increase in productivity of agricultural lands as conditions became congenial for irrigation. This might account for the expansion of population and the high development of culture at that time.

Cohonina population morements into the Grand Canyon and its side canyons are similarly grouped about the dates determined for the environmental shifts. Schwartz (1956) states that up to A.D. 900 there was no major use of the canyons as habitation areas, but that after that date the Cohonina movement into the canyon began. About 1100 nonCohonina peoples from the west and south "who up to this time had lived more or less permanently on the Plateau" moved in also. By A.D. 1200, the Cohonina had abandoned the Plateau entirely.

If the Cohonina and other peoples living in that area of the Plateau were primarily rain farmers it is understandable why movement into the Grand Canyon area would not have been advantageous earlier. The date of A.D. 900 for movement into the canyon is about 100 years earlier than we would suspect on the basis of the palynological information from the study area but not so much earlier as to discount the argument. The A.D. 1000 date is a probable one, as determined in this study, and Schwartz' A.D. 900 date seems to be of the same type. It has been estab- 
lished in this study that the environmental shift had been completed by the year 1200, however, and this would explain the abandonment of the Plateau by that date. The dating problem is complicated by the availability of rich farm lands in the Flagstaff arca resulting from the eruption of Sunset Crater in the middle of the eleventh century A.D. By 1160 , however, this resource had been made unavailable because of erosion (Colton, 1936. 1949) - a result which might be expected under the environmental conditions postulated.

Schwartz (1957) also considers that population movements of the Cohonina were due to changes in meteorological conditions. His arguments are developed upon different bases, but he concludes that peoples living on the Plateau practiced dry farming until the tenth century. Movement into the Grand Canyon and its side canyons is thought to have been neccssitated by a population increase coincident with a shift to greater aridity and to have been accompanied by a period of experimentation in architecture and agriculture. A period of erosional activity is recognized between A.D. 1200 and 1350 , perhaps triggered by a stormy period following a drought around A.D. 1100. The basic difference between the position of Schwartz and that advanced in this paper is that the meteorological changes are thought to have been due to different causes. Schwartz considers that the changes were due to a climatic trend toward greater aridity which had been going on since ca. A.D. 1. In this paper, changes in the summer rainfall pattern are thought to have created erosional activity and lowered water tables. Thus conditions effectively more arid were in effect at certain time horizons, though climatic change was not involved.

Woodbury's recent study (1961) of agricultural practices in the Point of Pines area notes that terracing systems were initiated about A.D. 1000. Strictly speaking, this area does not lie north of the Mogollon Rim, but its elevation places it within the steppe climatic zone. Since the Point of Pines area was inhabited long before A.D. 1000 by agriculturalists (Martin and Schocnwetter, 1960), why would terracing systems have been inaugurated only after that date? It secms reasonable to suppose that it was only after A.D. 1000 that some sort of erosion controls were necessary. According to the postulated envirommental conditions it was only after that date that erosion controls would have been neccssary.

For the Sinagua (Colton, 1946; Schroeder, 1960) a pattern similar to that recognized for the Cohonina is seen. After the lands produced by the eruption of Sunset Crater were no longer arable, a retreat to the valleys where permanent water was available is noted, in this case the 
Verde Valley. Irrigation was definitely practiced in the Verde Valley after A.D. 1250 (Schroeder, 1948).

From the preceding discussion it is clear that the postulated model of environmental changes may be used rather effectively in explaining certain cultural changes which have occurred in the steppe climatic zone. Of course this does not constitute proof of the model, but it does seem to argue for acceptance of the model as a starting 'point for future investigation.

One more major question remains. It is known that a great deal of territory within the steppe climatic zone was abandoned by agriculturalists after about A.D. 1400. While this may be explained as due to erosional activity which caused a reduction in crop potential during Period I-b, historical records (Hastings, 1960) and geological stratigraphy (Bryan, 1925) clearly show that the erosional cycle had ceased long before the nineteenth century. The pollen record of Period I-a gives indication that the pattern of summer rainfall was not disrupted, but if the river valleys were not dissected why was irrigation agriculture not once more highly developed in the steppe climatic zone?

It has been indicated by lexico-statistics (Hoijer, 1956) and archaeology (Riley, 1954) that Athapascan-speakers were probably established in the Southwest before A.D. 1300. Possibly the culture of the Athapascanspeakers on their arrival was adapted to a boreal forest environment, like that of the original homeland in the far north. Even today the culture of the Western Apache (Kaut, pers. comm.) is essentially adapted to boreal conditions. These people do plant a small crop of corn in favorable micro-environments in the spring, but the bulk of their economic supply comes from hunting and gathering in the pine forests of higher elevations during the summer. As they return to winter encampments in the warmer lowlands they stop off to harvest the crop which was planted earlier. The Chiricahua Apache (Opler, 1941, p. 374) plant near their summer encampments, leaving most of the cultivation to the women while the men are hunting.

If people following similar economic patterns were established in the area after its abandonment by the agriculturalists they might have served as a barrier to re-occupation when conditions in the river valleys changed. If aggressiveness and raiding were already established cultural patterns among the Athapascan-speakers, they might have been formidable opponents to sedentary puebloans.

Some small amount of data on environmental conditions existing after A.D. 1350 tends to support this tentative hypothesis. Pollen profiles show 
an increase in arboreal pollen in Period $\mathrm{I}-\mathrm{a}$. This might indicate that after its abandonment by agriculturalists the eastern-Arizona-westernNew Mexico area underwent an expansion in the amount of forested land. Since it is possible that the Athapascan-speakers were culturally adapted to boreal forest conditions at the time of their entry, the effect of forest expansion would have been to offer an expansion of territory for forest-dwelling pcoples.

It is recognized that an increase in the frequency of arboreal pollen may not be due to an increase in the number of trees. It is equally possible that it reflects a decrease in the output of some other pollen producer, such as cheno-ams, due to changes in environmental conditions which do not affect the trees at all. Noting the apparent invasion of trees at the Promontory Site and Site 30, however, and Woodbury's contention that forest movement took place at Point of Pines (Woodbury, 1961, p. 2), I consider it very likely that arboreal expansion has taken place.

Even an increase in forest need not be explained as a purely natural phenomenon. It is quite possible that the growth of forest was a re-grozeth reflecting the cessation of land clearance measures by the agriculturalists. It is recognized, though, that dendroclimatic records for the time period 1350-1800 (Schulman, 1956) indicate an environment favorable for tree growth.

\section{Appendix A: SAMPLE GOLLECTION TEGHNIQUE}

The profile or arroyo bank selected for sampling was that which showed the most stratigraphic detail. The section to be sampled was measured and mapped on graph paper when ordinary field notes would not be sufficient for a description. When possible, the section to be sampled was so located that wind-blown pollen and debris blew into the face of the collector and not into the samples he was collecting.

The section was measured vertically by means of a steel tape. Sampling intervals of 5,10 or $15 \mathrm{~cm}$. were selected relative to the depth and stratigraphic detail of the section. The intervals were marked off on the section by means of marks made with a trowel. The trowel was then wiped off so that no dirt was visible, and the surface of the face was scraped away to a depth of one-half or three-fourths of an inch at the lowest mark. Using the trowel as a scoop, a quarter to a half pound of sediment was removed from the level indicated by the mark and placed in a clean vinyl plastic bag. After the bag had been sealed and labeled, the trowel was wiped clean again and the procedure repeated for the next 
lowest mark, then the next, and so on. Sampling the section from the bottom upward reduces the possibility of contamination from upper levels.

\section{Appendix B: POllen ExtRaCTION TECHNIQUE}

\section{STEP 1}

A $50 \mathrm{ml}$. nalgene, round-bottom centrifuge tube is one-half to twothirds filled with sediment from a sample and pollen-free water added (distilled or filtered) to the three-quarter mark; $1 \mathrm{ml}$. of a solution of quebracho, $2 \mathrm{ml}$. of pine oil and $3 \mathrm{ml}$. of a solution of laboratory detergent are added and the whole is thoroughly stirred. The tube is placed into a large beaker and an air or gas source is inserted into the tube. When the gas is added slowly, oily bubbles are formed which carry nonsilicious material out of the tube and into the beaker. Water ${ }^{1}$ is added to the tube as it is depleted and the mixture stirred often during the process. After a half hour the detritus in the tube may be discarded and the tube cleaned and the material in the beaker put back into the tube and centrifuged. The supernatant is discarded. The mixture is then given a water rinse and centrifuged and the supernatant discarded. The quebracho acts to depress silica in aqueous solutions so the material bubbled into the beaker is largely silica-free (Arms, 1960).

\section{STEP 2}

A : Conc. $\mathrm{HCl}$ is added to the tube slowly with cautious stirring until evolution of gas ceases. This dissolves carbonates in the mixture, primarily, and makes the mixture acid to facilitate the next procedure. The mixture is allowed to sit overnight ${ }^{2}$ and is stirred again before centrifugation and discard of the supernatant.

$\mathrm{B}$ : Conc. HF is added to the tube slowly with cautious stirring until evolution of gas ceases. This dissolves almost all of the remaining silicates. The mixture is allowed to sit overnight and stirred again before centrifugation and discard of the supernatant.

$\mathrm{C}$ : Another rinse is given with conc. $\mathrm{HCl}$ to remove fluorides and to lessen the acidity of the mixture. The tube is centrifuged and the supernatant discarded.

D: The mixture is rinsed with glacial acetic acid to dehydrate the organic fraction. The tube is centrifuged and the supernatant discarded.

${ }_{1}$ All water must be pollen free to avoid contamination. Equipment must be rinsed with such water before use.

2 Whenever the mixture is left the open surface of the tube must be covered to avoid contamination by modern pollen. 
E: $10 \mathrm{ml}$. of acetolysis mixture (one part of sulphuric acid to nine parts of acetic anhydride) are added to the mixture slowly and with cautious stirring. When the erolution of gas has mostly ceased, the tube is transferrecl to a hot water bath for five minutes. This procedure destroys a considerable portion of the organic fraction of the mixture but does not act on the pollen if carefully done. The tube is centrifuged and the supernatant discarded.

F: The mixture is rinsed with glacial acetic acid to stop the preceding reaction and to make the mixture less acid. The tube is centrifuged and the supernatant is discarded.

G: The mixture is given two rinses with acetone to remove any materials that might be soluble in this solvent and to prepare the mixture for the next step. The supernatants are discarded.

\section{STEP 3}

A: $15 \mathrm{ml}$. of flotation mixture (two parts of tetrabromocthane to one part of acetone) are added to the tube, a stopper is put into the tube, and the whole is shaken thoroughly; then the tube is centrifuged at halfspeed for 15 minutes. The flotation mixture has a specific gravity of about 2.0 and so will separate the lighter fraction containing the pollen from the heavier fraction of the mixture, which will sink to the bottom of the tube. The supernatant is decanted into a clean centrifuge tube; the heavier material is discarded.

B: Acetone is added to the supernatant and the whole thoroughly stirred. This reduces the specific gravity so that the pollen-bearing debris can be centrifuged to the bottom of the tube, after which the supernatant is discarded.

$\mathrm{C}$ : The mixture is given another rinse with acetone to insure that all flotation mixture is remored. The tube is centrifuged and the supernatant discarded.

D: 10 per cent $\mathrm{KOH}$ is added to the mixture and the tube transferred to a boiling water bath for ten minutes. Frequent stirring aids the reaction, in which certain of the remaining organic materials are destroyed and the polten is expanded for better microscopy. The tube is centrifuged and the supernatant discarded.

$\mathrm{E}$ : The mixture is transferred to $10 \mathrm{ml}$. shell vials with a jet of alcohol and centrifuged. The supernatant is discarded.

$\mathrm{G}$ : Enough glycerin is added to the mixture to cover and keep it moist. A water solution of basic fuchsin stain is added before viewing the 
material. An alternative is to add pre-stained glycerin jelly to the vial rather than glycerin and stain. 


\section{Summary}

During the field season of 1960 several projects were undertaken:

1. Archaeological excavations of six sites.

2. Continuation of the archaeological survey of the Upper Little Colorado River Drainage.

3. A paleoecological inquiry by means of pollen analysis.

The costs of the palynological research, of the archaeological survey, and of the excavation of the Tumbleweed Canyon Site were paid for by means of a grant from the National Science Foundation. We are grateful for their assistance, without which the scope of our archacological activities would have been sharply limited.

The sites, briefly summarized, are given in chronological order, with the earliest first. Then follows a section concerned with Settlement Patterns and various conjectures derived from these; and finally a brief resume of the results of the other three major projects: the study of pottery designs; the archaeological reconnaissance; and the paleoecological study.

\section{TUMBLEWEED CANYON SITE}

The earliest excavated site, Tumbleweed Canyon Site, consists of three pithouses and three circular pits that may have been used for storage. This site was located on a high mesa-top overlooking Lyman Dam and the Little Colorado River Valley. Crude, double walls made of lava boulders span each end of the long, narrow mesa and also span any possible means of access. It is possible that these "walls" served as primitive defense systems to exclude unwanted visitors (see Chap. VII, "Conclusions," for a notable discussion of this).

The types of stone tools that occurred at this pithouse village include the following: one-hand manos, basin metates, rubbing stones, cylindrical pestles, choppers, notched or shouldered projectile points, flake knives, and scrapers. It should be noted that these types are also found at later villages. We would be hard pressed then to single out one of these types of stone artifacts as the type characteristic of an early phase (that is, before A.D. 500). We can say, however, that the types listed above occur more abundantly in early sites and tend to decrease in frequency or pop- 
ularity in sites of later dates. In other words, we do not regard types of stone tools as sensitive chronological indicators. It is usually conceded that they are less useful than pottery, for instance, in detecting more exact chronological divisions.

The study and analysis of stone artifacts, especially from a pre-ceramic site like Tumbleweed, are important, however, in any functional applications of archaeological research, the essential purpose of which is to provide information about man's past. At best, the archaeologist is hampered in his attempt to learn of past cultures and societies because only a portion of the whole culture survives. We must utilize all available data if we hope to learn anything about the past.

Tools of stone are among the few classes of imperishable objects to survive in unchanged form. Stone artifacts are diversified and enter into nearly every aspect of the life of the people. From them we can often make guesses concerning agriculture, hunting activities, preparation of food, ornamentation, house building, and even ceremonial activities. From these data we may be able to create hypotheses concerning the growth and development of culture, culture change, trade relations, the specific tradition to which a given site belongs, and perhaps even social organization. It is the study of minutiae of the material culture of a past society which makes it possible to interpret archaeological data-specifically, artifacts of stone, of bone, of pottery and of architecture-in terms of social history.

The stone artifacts from Tumbleweed Canyon Site, then, are not complex when compared with those from sites that were occupied in A.D. 1200, for example, but they are exceedingly useful, nevertheless.

Diligent search, however, produced not a single sherd of pottery. We assume, therefore, that the people who built and lived in the crude shelters that we investigated did not make pottery. This is a curious anomaly that is not readily explainable, since contemporary peoples some hundred miles to the south did manufacture pottery. Did the dwellers of Tumbleweed Canyon Site know of pottery-making and refuse to accept this art, or were they so isolated that knowledge of this art had not yet reached them?

Charcoal from the roof(?) beams was dated at the Laboratory of the University of Groningen, The Netherlands, by Dr. H. de Waard at 1685 years before the present, \pm 50 years (GRN 2801). "It is possible that the value found by C-14 method may be in error up to 200 years. The maximum error, however, is rather improbable." (Letter from Dr. de Waard, August 13, 1960.)

We date the occupation of this site at about A.D. 275. 


\section{GOESLING SITE}

Two pithouses were dug out of a possible ten or more. Both had burned and one had subsequently been used as a trash dump. Nearly 5000 sherds were recovered from this site. Of these, over 25 per cent were classified as Red Mesa Black-on-White sherds. Drs. E. B. Danson, Emil W. Haury, Alan P. Olson, Raymond H. Thompson, William W'. Wasley, and Fred Wendorf, all of whom are familiar with this type of pottery, would probably classify these sherds as bearing designs in a Red Mesa Black-on-White style.

The significance of the site lies in the fact that the abundance of large black-on-white sherds presented us with an excellent opportunity to institute a comparative study of design elements on the Red-Mesa-style sherds and on those that we are calling Snowflake Black-on-White (see Chap. III).

No tree-ring or carbon-14 dates have been obtained from this site. An estimated date of A.D. 900-950 is placed on it.

\section{CHILCOTT SITES}

The three Chilcott sites include several surface rooms with masonry walls, one pithouse, and several brush shelters. The pithouse and its antechamber were subsequently remodeled into two rooms. Firepits occurred in several rooms. From these sites, we obtained a total of 2600 sherds. The most popular painted type was Snowflake Black-on-White (19 per cent of all sherds) with Reserve, Tularosa and Kiatuthlanna Black-on-White trailing behind.

The date of these sites is placed at about A.D. 1200 (Groningen GRN 2414) (760 \pm 80 before present).

\section{THODE SITE}

Eleven rooms out of a possible twelve to fifteen were excavated. The village plan or arrangement of rooms and the primitive masonry were of special interest to us because they probably represent a step in the development of pueblo architecture and arrangement of rooms. The rooms were of irregular shape, with walls of crude masonry composed of unshaped cobbles and boulders of varying sizes. Floors were semi-subterranean, from 10 to $30 \mathrm{~cm}$. below the old ground surface. Interior equipment was limited to a firepit in one room, possible hearth areas in two other rooms, and a few milling stones. Under the wall of one of the units two burials, with mortuary pottery, were located. No kiva was found. 
Sherds were small in size and few in number. Only 765 were recovered, of which over 60 per cent were decorated types. The most popular type was Snowflake Black-on- White (23 per cent of the total). Following it in decreasing order of popularity were: Tularosa Black-on-White (4 per cent), Reserve Black-on-White (2.61 per cent). and Kiatuthlanna Blackon-White (2.10 per cent). There were also three sherds of Wingate Black-on-Red.

The estimated date for the Thode Site is put at A.D. $1200 \pm 50$ years.

\section{RIM VALLEY PUEBLO}

The plan for this village approaches that found in later villages. The rooms are grouped in two units on opposite sides of a plaza. Eleven rooms out of a probable twenty-five were cleared. The rooms are rectangular, with walls of vertical-slab masonry or random rubble composed of cobbles. The doorways that were found were in partition walls. No doorways leading outside were discovered. Interior equipment consisted of firepits, wall ventilators opposite the fireplaces, and flour receptacles. No subterranean kiva vent was found, but certain features of Room C suggest that it may have been used for ceremonial as well as secular functions.

Pottery types arranged in a descending order of occurrence are: Reserve Black-on-White (7.59 per cent). Snowflake Black-on-White (6.03 per cent), and Tularosa Black-on-White (4.62 per cent). A sprinkling of the 2188 sherds found have been classified as Wingate Black-on-Red. Houck Polychrome. Tularosa White-on-Red and St. Johns Polychrome.

A tentative date of about A.D. $1225 \pm 50$ years has been assigned to this site.

\section{GREAT KIVA, HOOPER RANGH PUEBLO}

The pueblo to which this Great Kiva belongs has been described in detail (Martin, Rinaldo, and Longacre, 1961; see also p. 220 of this report).

The Great Kiva is one of the largest rectangular kivas to have been dug. It measures 15.5 by 14.5 meters (interior dimensions). Entrance is by means of an easterly-oriented ramp that slopes gently downward into a vestibule. A large sandstone slab set on edge between the firepit and the ramp-entrance acted as a deflector - an unusual feature in a Great Kiva. The interior of the kiva was provided with a bench on all 
sides, and two vaults flanked the central area and the firepit. The vault on the south side of the building was lined with masonry, whereas the north one was simpler.

In cleaning the floor west of the south vault, we removed a small slab) of sandstone that may have been "sealed" in place by a calking of adobe and that had been placed over the annular opening of a ring slab. A glance through the opening indicated that little dust had collected in the box or crypt and that it contained two objects - a human effigy of sandstone, carved and painted, and a miniature jar. In the jar and on the floor were beads of several colors. The figurine, lacking the right arm, was face down. The front of the sandstone figure had been carved and painted to represent, perhaps, a supernatural being. For a detailed description and a full discussion of its possible significance, see Chapter II. The crypt appears to duplicate in miniature the Great Kiva itself and the annular slab may represent the opening to the kiva. Thus the crypt may be symbolic of a kiva and the entrance to the underworld.

At present, we regard this Great Kiva as a product of converging traditions, derived in part from Mogollon ceremonial structures and in part from Anasazi great kivas. The convergence of Tularosa Mogollon and Chacoan Anasazi traditions is likewise manifested in the ceramics found at Hooper Ranch Pueblo.

Among the decorated types of pottery the most popular ones, in descending order, were Tularosa Black-on-White (11.00 per cent), Wingate Black-on-Red (6.77 per cent), St. Johns Polychrome (3.13 per cent), Heshota-uthla Polychrome (2.08 per cent), Four Mile Polychrome (2.02 per cent), and Kivakina Polychrome (1.88 per cent). There was also a sprinkling of the Pinnawa series.

When in the lifetime of the Hooper Ranch Pueblo was the Great Kiva built? Architectural data alone could not answer this important question.

We turned, therefore, to other available evidence.

Schoenwetter (Chap. VIII) suggests, on palynological grounds, that the Great Kiva was built during the latter days of the pueblo. Freeman (Chap. IV), in his statistical analysis of the painted pottery from some of the rooms and from the Great Kiva at Hooper Ranch, likewise places the Great Kiva as "late" in his scriation. He states, however, that the Hooper Ranch Pueblo afforded the most dubious of any analysis that he constructed in this report.

My colleague, Dr. Rinaldo, and I agree with the supplementary findings of our collaborators, and in the absence of absolute dates or other evidence to the contrary, we accept the hypothesis that the Great Kiva 
was built, or at least used most frequently, in the last days of the occupation of the village.

What conjectural date do we place on the Great Kiva?

In our report on the pueblo itself (Martin, Rinaldo, and Longacre, 1961) we estimated the dates of the pueblo as running from about A.D. 1200 to 1375 .

Charcoal from the floor of the Great Kiva has been dated at Groningen at $730 \pm 60$ years before the present or A.D. 1230 (GRN 3006).

\section{SETTLEMENT PATTERNS}

Although six sites were excavated, they are somewhat widely separated in space and time. Not too many firm conclusions can be based on this kind of checkered information. A few brief inferences may perhaps be permitted, especially if they incorporate our other evidence from the area.

\section{Tumbleweed Canyon Site}

The pre-pottery pithouse site at Tumbleweed Canyon is presently mique since it is the only excavated one in our area. In the course of his survey Longacre found a few pre-pottery sites with one or two depressions (pithouses?) outlined by boulders (LS sites 84 and 86; Martin, Rinaldo, and Longacre, 1960). These may be sites similar to and coeval with Tumbleweed Canyon Site.

One can hardly speak of a village "plan" or "organization" where only three houses are involved! We can stress their isolation, located as they are atop a mesa several hundred feet above the river. Water may have been available in now dry springs; if not, water had to be carried up from the Colorado River. Corn was grown or imported, for corn pollen was found (Chap. VIII). If corn was grown locally, the fields may have been down on the flats near the river, for it would not have flourished, in all probability, on the mesa top unless the rainfall pattern was different. The economy of the group was apparently a combination of hunting, plant-collecting and some agriculture (corn). The presence of storage pits must be emphasized. It is probable that the population was sedentary. Baking ovens were not encountered, but since firepits were found, it is assumed that cooking, parching or roasting may have taken place within the house.

It is really impossible to make any guess as to density of population for this time period of about A.D. 275. Based on available evidence, I should assume that the regional population was very small. 
Nuclear families may have inhabited the pithouses, perhaps three persons to a house. This random placement of several houses certainly suggests nuclear families rather than an extended family unless an extended family occupied all three houses. With this kind of village arrangement I should assume that each village represented a politically independent and endogamous band.

Favored locations for pre-pottery pithouses include several topographic categories: on mesa tops; on a bench on the side of a mesa; and on a ridge in a valley floor.

\section{Goesling Site}

The two pithouses excavated at this site (out of perhaps 10 to 15) belong to the Anasazi tradition. The village to which they belong was apparently composed of independent dwelling units arranged in no particular order. Storage-cists and refuse-areas were prohably present but we did not look for them. One might refer to this site as a multiple-unit site consisting of several pithouses and additional features typical of a Pueblo I or Pueblo II village. The village was located on a low terrace overlooking the valley of the Little Colorado River. Water may have been drawn from springs, if they existed, or from the not too distant river. Corn, and perhaps beans and squash, were probably cultivated in the flood plain - a very short and convenient distance from the village. The economy of the people of this village was largely based on the cultivation of crops.

I should guess that nuclear families constituted the social organization of villages of this type and time period. From the survey, we know that in our area of study there are probably 60 sites that belong to this period (about A.D. 900). If one assumes that there are, on the average, five dwelling units per site and that three people lived in each dwelling, one finds that there may have been approximately 900 persons living in this area at this time. While such an estimate of population is perhaps rash, I should explain that I do not take this or any of these estimates literally. They merely rank the populations of the villages of differing time periods in a relative order and give one a sketchy impression of population change.

\section{Chilcot't Sites}

Although we named three sites "Chilcott," we feel that only Site 1 was the home village. It is possible that Sites 2 and 3 served as farmhouses or store rooms, since they appeared to have been brush shelters rather than permanent dwellings. My remarks, then, will pertain to Site 1. 
Two rectangular rooms in this unit were contiguous, but the other three were close by but separate structures. The walls of the rooms were composed of masonry of a crude rubble type and may represent some of the earliest masonry in our area. It should be clear, however, that the three rooms were not contiguous. Each room was a separate unit and the units were clustered near to one another. This village probably represents an early stage in the development of pueblos, but one can hardly state that the germ of the later village plans lies in a hamlet of this period. In fact, some of the rooms possess floors that were excavated somewhat below the old ground surface and, in this characteristic, retain a pithouse flavor. But the walls, composed of odd boulders, were footed on the surface of the ground and continued upward. In other words, the lower segment of wall was of native earth with no veneer of masonry.

The source of water for this village is not known. We suspect that crops were grown and that the economy was agricultural with little dependence on food-gathering. Some animal bones were recovered, and this fact permits us to assume that hunting and trapping were of moderate importance. Since pottery was present, some cooking was probably done in pots; but some plant and game may have been roasted.

The social organization may have consisted of one or two extended families per village, matrilocal residence, matrilineal descent and inheritance, and possible exogamous clans.

\section{Thode Site}

Most of the foregoing remarks on Chilcott Site 1 would apply here. The eleven rooms were neither rectangular nor round. The floors were semi-subterranean, perhaps 20 to $40 \mathrm{~cm}$. below the old ground level; the base of the masonry walls was on the old ground surface; and the masonry was composed of unshaped cobbles and boulders set in mud mortar. (How such walls carried the weight of a roof is a mystery to me!) The rooms were clustered near one another but were not contiguous. The arrangement of the rooms makes one think of the informal, unplanned order of a pithouse village, where each unit is independent of the others. In this instance, the rooms are closer together, with perhaps only $50 \mathrm{~cm}$. separating them. The people seem to have drawn eloser together. Here one can sense the beginnings of pueblo architecture in which the rooms at a later date are arranged in cellular fashion. There was no "front" or "back" to the Thode Site. No ceremonial room was found.

Water for domestic purposes may have been taken from Mineral Creek. Agriculture may have been practiced although we have no direct evidence of it. The flood plain would have provided ideal soil and a 
convenient area for fields. Game probably supplemented the vegetable diet. Food may have been cooked in pottery jars or roasted and broiled over the fires. Two rooms contained hearth areas and one contained a firepit.

Sites belonging to this time period (Chilcott and Thode) are fairly abundant and may represent about one-third (85) of all the sites found on the survey. A guess as to the population of this period (with a minimum figure of three people per room and ten dwellings per site) would produce an estimate of about 2600 persons for the period of A.D. $1200 \pm 50$ living in approximately 1500 square miles (Springerville-St. Johns, Snowflake-Show Low). This estimate is arbitrary, perhaps a bit low.

The survey indicates that the people living in this period (during which time separate, above-ground rooms with masonry walls were being placed closer together but not yet in contiguous fashion) preferred (by about 58 per cent) to build their villages on a knoll or the floor of a valley adjacent to a stream. Others preferred a point of land jutting into a valley, a bench on the side of a mesa, or the edge of a mesa overlooking a stream.

The social organization for the Thode Site may have been similar to the Chilcott Site: several extended families occupying the village; matrilocal residence; matrilineal descent; and possibly exogamous clans.

\section{Rim Valley Pueblo}

The rooms of this village are rectangular or square, are contiguous, and are grouped in two units facing each other on opposite sides of a plaza. This site and another, Mineral Creek Site (Martin, Rinaldo, and Longacre, 1961), are among the earliest examples we know in this area of a "pueblo" in the sense that the rooms are contiguous and the floors are at ground level.

At Rim Valley, the pueblo has no "front"; the rooms are linear agglomerations. The rooms face inward toward the plaza. The arrangement of rooms seems typical of Mogollon architectural development; that is, a rather unsystematic cluster of square or rectangular rooms. To put it another way, if one were a person of Paul Bunyan's stature and if one were to square up the amoeba-shaped rooms of the Thode Site (see fig. 21) and were to push them together so that the roons were contiguous, one would have a graphic representation of Mogollon villages. In fact, even some of the later sites, such as Jewett Gap Pueblo (personal visit), Foote Canyon Pueblo (Rinaldo, 1959) and Kinishba (Cummings, 1940), as well as some of the pre-Spanish Zuni towns, are really nothing 
but exaggerated counterparts of the earlier Mogollon villages-groups of rooms pulled together and irregularly disposed. They are simply extensive house blocks or groups of contiguous rooms, in some cases of more than one story, but now distributed around a plaza and/or kiva.

Thus, Rim Valley Pueblo is an excellent example of one of the earliest types of Mogollon villages and exhibits characteristics that hint of later developments, such as one finds at Hooper Ranch Pueblo (see below). Although I have conjectured that a span of perhaps 20 to 50 years separates the Thode Site (A.D. $1200 \pm 50$ ) from Rim Valley Pueblo (A.D. $1225 \pm 50$ ), this is nothing more than a guess and perhaps is misleading. The seriation of Rim Valley Pueblo sherds was not satisfactory, but Freeman suggests (Chap. IV) that the site may be slightly later than the Thode Site.

On the basis of architecture and village plan, I tend to agree with this position. But the Thode Site and Rim Valley Pueblo may be nearly of an age. My reason for advancing this paradoxical suggestion is twofold: (1) Rim Valley Pueblo is on the Little Colorado River and therefore may have been in closer contact with more sophisticated developments that were going on elsewhere. The Little Colorado River Valley was undoubtedly a route of travel and trade, and peoples living there would have been, generally speaking, subject to tendencies of urbanization and more exposed to the latest fashions. (2) At the same time, the folk of the area in and around the Thode Site lived in a remote, isolated area, cut off from innovations. For this reason, the architecture of the two sites differs, Rim Valley being more "modern" while the Thode Site is more antiquated or vestigial; but the pottery types are essentially similar.

This interpretation reverses our usual attitude towards pottery, which generally is regarded as the sensitive diagnostic criterion for defining and dating minor chronological divisions of prehistory.

I can venture no other explanation for the apparent differences in two sites that probably were contemporaneous or nearly so; unless, of course, my chronology is erroneous. The fact remains, however, that the pottery types that are generally regarded as good time markers-Reserve, Snowflake and Tularosa Black-on-Whites and Wingate Black-on-Redare present at both sites, though in slightly different proportions.

Our investigations at Rim Valley Pueblo reveal no kiva-building that was detached from the main blocks of rooms. Since the pucblo was built on bedrock which was everywhere exposed or was, at best, covered with only a few inches of soil, it is unlikely that we missed it. Separate kivabuildings often seem to be absent from pueblos of this period. It is quite possible, however, that some of the dwelling rooms might have doubled 
as places for small group ceremonies. In Rim Valley Pueblo, for example, Room C might have been a place in which ceremonial and secular functions could have been carried on. This room is furnished with a firepit, a ventilator, a ladder-pit(?) flanked by stone slabs, and the kind of vault or foot-drum in which one often finds a sipapu. These furnishings, which are more elaborate than those usually found in elwelling rooms, have been found elsewhere (see Chap. I, "Summary of Secular Architecture") and persuaded us to wonder if this room might not have served also as a kiva.

Water for domestic usage for Rim Valley Pueblo could have been obtained from the Little Colorado River several hundred feet below. If nearby springs existed, no sign of them remains.

Although we discovered no evidence of cultivated foodstuffs (corn, beans, and squash), it is assumed that these crops were grown, probably in the flood plain of the river that flowed below the houses. Animal bones were found in some quantity, a fact that makes it probable that hunting supplemented farming. Firepits were probably used for boiling, stewing, parching, and roasting vegetables and meat, and for heat and light. These firepits are centrally located in the rooms, are sometimes associated with ash pits (the need for and meaning of which are not clear), and are cunningly placed near a ventilator opening set in the walls.

The favored locations for villages of this class and era and an estimate of the population for the area under study will be stated in the discussion of Hooper Ranch Pueblo.

\section{Great Kiva, Hooper Ranch Pueblo}

The village at Hooper Ranch was a "true pueblo," an agglomeration of contiguous rooms clustered about three sides of a plaza that contained the two lesser kivas (Kivas I and II in Martin, Rinaldo, and Longacre, 1961, p. 32). The village arrangement is similar to Kinishba (Cummings, 1940, map) and other plaza-type (Hawikuh-like?) towns.

There were two stories in the pueblo but we have described them as consisting of two horizontal levels of occupation, not contemporaneous, each containing perhaps 60 rooms. Our evidence indicates that the upper story was built and occupied only after the lower one was abandoned and filled with relatively clean dirt (containing few sherds or artifacts). This does not preclude the possibility that there were two stories during the period of first occupation; but we have little or no evidence to support such an idea.

It is probable that the rooms faced inward toward the plaza. Whether this orientation of the village reflects a different kind of social organization; 
a tighter kind of village control; a more conservative (inward-looking) village character; a desire to obstruct the view of "foreigners" or traders, who might otherwise profane the sanctified enclosure of kivas by their glances; or whether it was an attempt to make the village defensible or cozy, I cannot say. This ruin exemplifies in miniature the fruit of MogolIon architectural development that required about a thousand years to germinate.

The dating of the Hooper Ranch Pueblo is conjectural and not too satisfactory. We have guessed that the pueblo was constructed and occupied from A.D. 1200 to 1375. A carbon-14 date from the Great Kiva is A.D. $1230 \pm 60$ (GRN 3006). The earlier portion of the village may be contemporary with some of the dwelling units in Rim Valley Pueblo.

Water for the use of the village was certainly not a problem. The Little Colorado River flowed within 25-75 feet of the pueblo and was, in all likelihood, a perennial stream, as it is today.

The economy of this site is fairly well worked out from the pollen study conducted by Schoenwetter (Chap. VIII). Pollen from corn and squash was identified for the earlier or lower level of occupation. The pollen of cattail was recovered, and this indicates a nearby swamp or marshy area. In the later stages of the life of the pueblo, the percentage of corn pollen decreases. This shift plus others (discussed in Chap. VIII) may indicate a cultural change or a small fluctuation in rainfall pattern. An unusual quantity of animal bones in the refuse suggests that the diet was supplemented to a considerable extent by meats. The presence of firepits implies, as it did in the other sites, cooking of various kinds- broiling, boiling, parching, roasting - and a source of light and heat.

Sites of the Rim Valley and Hooper Ranch Pueblo class (38 found on survey) are less numerous than those in the Chilcott-Thode Sites class (85). On the whole, most of them (32) are found in the Little Colorado River Valley and the Snowflake-Mesa Redondo areas, probably because permanent streams were present and crops could be grown nearby. If it can be assumed that each of these villages averaged about 25 rooms (probably a low average) with probably three people per room, then I should guess that the population may have been 75 to 100 people per village: and if this figure were multiplied by the number of known villages of this era (38), it would indicate that approximately 3800 people were living about A.D. 1100-1300 near the Litule Colorado River and its tributaries near Snowflake and Mesa Redondo. I have not forgotten that not all "rooms" were dwelling rooms, that some were used for storage purposes; but I think this fact would he more or less offset by the fact that most villages of this period (A.D. 1100-1300) may have contained from 50 to 60 rooms 
each. My figure for density of population is probably inexact, but, if anything. it may be low. As stated earlier, I regard this sort of guessing concerning population as useful only in ranking the approximate number of people per century within our study area. It may be relatively correct, but is not intended to be a census guide.

The social organization of the Rim Valley and Hooper Ranch Pueblos may be conjectured to have been similar in many aspects to that of the towns of preceding centuries. In fact, as we approach the contemporary western pueblos in time, we may be on surer ground in putting forth our guesses. The "inward-looking" pueblos may not have been very differcent from what we can observe in Kuni and Hopi towns.

It is probable, then, that within the large puelslos we might find several matrilocal families, matrilineal descent and inheritance, and a more complex grouping.

The absence of an arrangement of structures that would indicate moiety divisions suggests that there existed a single organizational unit with perhaps several fraternities that controlled the kivas and the ceremonies held within them. The presence of the Great Kiva might signify some sort of supra-village organization made up of priests from satellite towns who were responsible for the major rites held within this eminent and august structure.

The remainder of my chapter should, perhaps, have preceded the summary on the dig and the section on settlement patterns. Or, more logically, the parts that follow should have been interwoven into my summary, since my statements concerning settlement patterns and the conjectures stem from the next sections.

In truth, I could not have created many of my statements nor have derived hypotheses (climate, crops, number of sites per era, density of population, and the like) without having liberally drawn on the supplementary data provided by my colleagues. I owe them much. But I feared that the significance of these superlative reports would have been buried or wasted. For better or worse, I chose to treat them otherwise.

The results of three other projects remain, then, to be summarized. Two of these the Archaeological Survey Program and the Pollen Analysis Program were part of our original plan for the 1960 season. Both of these were financed by a generous grant from the National Science Foundation. I shall deal with these last. 


\section{ANALYSIS OF ELEMENTS OF POTTERY DESIGN}

A third project was not conceived until the last weeks of the field season. The black-on-white sherds from the pithouse at the Goesling Site (Pueblo I or II) were abundant (final total of decorated sherds about 2000). As we slowly perceived a possible relationship between the designs on them (Kiatuthlanna and Red Mesa Black-on-Whites) and what we had been calling "Snowflake Black-on-White" during the scason, we decided to institute a comparative study and analysis of the designs on these three pottery types. It was hoped that if a genetic relationship existed, we might be able to confirm it.

Miss Constance Cronin, assisted by Mr. Walter Boyer, then artist in the Department of Anthropology at the Muscum, undertook this comprehensive undertaking. In the Preface (pp. 5-6) I have described the preliminary aspects of this study.

Several striking and unexpected consequences flowed from this study. The principal one is the suggestion that Snowflake Black-on-White pottery designs are genetically closer to those on Kiatuthlanna Black-onWhite than to those on Red Mesa Black-on-White. I had assumed that since the pottery called Red Mesa Black-on-White was or might be closer chronologically to Snowflake Black-on-White, a closer relationship would exist between these types.

Since this study was made, several cxperts have looked over our sorting of sherds that we classified as either Kiatuthlanna or Red Mesa Black-on-White. I got two impressions from their remarks: (1) that our sherds classified as these types were not truly good representatives of Kiatuthlanna or Red Mesa Black-on-White types; but that one could say some were in the Red Mesa style and others in the Kiatuthlanna style (thus, we did not have "pure" types with which to work); and (2) that many sherds that we called Kiatuthlanna types, they would have classed as Red Mesa.

These observations may modify the conclusions of Miss Cronin but do not destroy them. A relationship between designs on early Chacoan ceramics and Snowflake Black-on-White is a possible conjecture.

Since Miss Cronin made her study and wrote her report, I have seen more Snowflake Black-on-White pottery. In addition to the link between the designs on Snowflake Black-on-White pottery and those on the two early Chacoan types, I can see the same kind of tie-up between the former and the early Kayenta pottery types. This is not startling, because I think most Southwesterners recognize a basic similarity between the design elements and design layouts of early Kayenta and Chacoan wares. 
At present, then, the origin, afliliation, and lineage of Snowflake Blackon-White pottery are not precisely known, but it seems reasonable to conclude that this type derives from the Chacoan and/or Kayenta wares.

\section{THE ARCHAEOLOGICAL RECONNAISSANCE}

Many results may stem from an archaeological reconnaissance. One may hope for clues as to climate, drainages and terrains, choice of settings for sites, arable land, and geological and ccological features. The surveys conducted by Rinaldo (unpublished) and by Longacre (Martin, Rinaldo and Longacre, 1960) have given us invaluable data. Using these, we can, among other things, intelligently choose sites for extensive excavation and we can make guesses concerning population density per unit of time. Without such source information, we should be working blindly.

Our study encompasses a large geographical area more or less rectangular in shape. The unit embraces the region from Springerville to St. Johns, to Snowflake to Show Low, Arizona, plus an extensive tract in the White Mountains. Approximately 1500 square miles are contained in these areas and 243 occupational components were found. In time, these range from ca. 1300 B.C. to A.D. 1300.

From the data given, it is possible to make some inferences.

The favorite location of the prehistoric peoples, regardless of time, was on a knoll or a ridge on the floor of a valley.

The density of population for the area studied increased from a probable few hundreds per century in pre-pottery times to perhaps about 3800 to 4000 during the centuries from A.D. 1100 to 1300 , and then declined until about A.D. 1500 after which time the area was deserted (unless the Apaches had moved in by this time).

The sites of the pre-pottery era (before A.D. 300) were found in all gcographic positions except in the White Mountains. By A.D. 900, the central area was almost vacant, but an increase of sites is noted along the Little Colorado and Show Low-Silver Creek drainage and in the White Mountains. It may have been the time when a lot of people were moving into the Point of Pines region south of the White Mountains; or it may have been a desire on the part of the people to find a location where sufficient moisture would permit them to farm.

About A.D. 1100-1300, most of the people were living on the two drainages of the area, with only a few remaining in the White Mountains, and soon after A.D. 1300 there were only a few large villages left in the area (six sites in all); these are all on the Little Colorado River or the Silver Creck River and a few major tributaries. 
Schoenwetter's palynological inquiry (Chap. VIII) supplies possible explanations for this ebb and flow and abandonment.

During the period that lasted from about A.D. 300 to 1000, the whole area under study may have been blessed with light summer rainfalls and heavier winter precipitation. The reserves of ground moisture were probably greater at that time: and streams - not only the two large ones still flowing in the area but also many small ones-flowed the year around. These stream beds are now only deep-cut, dry arroyos. At about A.D. 1000 and since. the area has received heavy, destructive, torrential rains such as it has today, and probably little of the gentle winter precipitation (see Chap. VIII for further explanations).

In a rery rough way, a correlation can be seen between the density of population and location of sites that were worked out in Longacre's survey, and a shift in environment. After A.D. 1000 or 1100 much of our area was inhospitable, and farming was difficult, if not impossible. The results? The people mosed to be near the two remaining streams; and after A.D. 1400-1500, life became too difficult and the people moved out. Where they went to is another question and it is being investigated.

The survey, then, provided us with data without which we could neither function as excavators nor serve as interpreters of the evidence dug up.

\section{POLLEN ANALYSIS}

I have found the interdisciplinary co-operation - the palynological inquiry by Schoenwetter (Chap. VIII) - eminently worth while. exciting. and satisfying. We are continuing this approach and hope to expand it and to use other interdisciplinary studies. It is immensely profitable, because data thus provided by our colleagues supplement ours and are essential for providing us with means to interpret the material culture we dig up.

I have no intention of trying to summarize the pollen project, for this would create needless bulk and tiresome repetition. Schoenwetter"s "Conclusions" and "Interpretations and Inferences" are admirably stated. I have already drawn on his data for some of my conjectures.

I shall content myself with drawing attention to a few points that interested me.

The main one is that a shift in environmental conditions may have occurred at about A.D. 1000. The "shift" to which reference is made means a seasonal change in amount of precipitation without altering the total annual rainfall. For example, at present, the Southwest as a whole 
receives the major amount of its precipitation in the summer months. Summer rains are dynamic and destructive because they fall in great volume in a short time and with great intensity. Such heavy torrential rains ("gully-washers" as they are locally called) cause old streams (now dry arroyos) to run furiously for a few hours. Arroyos are deepened and widened by such action. In addition to carrying off top soil and dissecting the lands and mountain sides, most of the water rushes off downstream and is carried off to the Little Colorado and to Lake Mead so quickly that little soaks into the ground. Thus, wells are not replenished, the water table is lowered, springs dry up, and the trees and plants profit very little from these summer thunderstorms.

Conversely, precipitation that occurs during the fall and winter months tends to be entirely beneficial. The rains are gentle and often of several days' duration; the snows, of course, are better than rains, even though they may not remain on the ground more than a few hours or days. The melt therefrom soaks into the ground and is entirely constructive. It is believed that winter precipitation replenishes wells and mountain springs, tends to perpetuate streams, and keeps the water tab]e high. It is possible, of course, to have the annual precipitation evenly divided, more or less; luut even under this condition winter precipitation is more useful to plants, animals, and man. Thus a "shift" in the pattern of rainfall may mean a change from dominant winter precipitation to dominant summer precipitation: or vice versa; or from one of these maxima to rainfall that is more or less evenly divided up throughout the year.

It is postulated that the shift in the microclimate in the Vernon area (at least) that occurred about A.D. 1000 was not a favorable one. The evidence at hand suggests that the rainfall pattern of prevailing winter precipitation shifted to one of preponderant summer precipitation. This spelled doom to the farmers, many of whom were in a "marginal" position.

Here, then, one has a possible explanation of the movement of peoples and of the abandonment of certain areas previously occupied. A cycle of preponderant summer rainfall would create an unfavorable environment for growing crops. Perennial streams probably disappeared and springs dried up. The explanation of these conditions is clearly given by Schoenwetter (Chap. VIII, pp. 194-195).

Relinquishment of certain areas has been noted in our archaeological work in the Pine-Lawn-Reserve, New Mexico, areas and also in our researches in the Vernon area. It is assumed that the people moved to nearby but more favorable places. To me this is most satisfying because 
we now appear to have a possible explanation for such movements of people - an explanation that is based on observation and good deductions rather than on wild guesses.

From the evidence at hand, four major periods of differing environmental conditions can be recognized from the pollen data. These periods are characterized by differing frequencies of pollen types, not by absolute differences in them. The four periods of different environmental conditions are vividly and briefly shown (Table 21, p. 199), and ample defense for Schoenwetter's point of view is given.

In his final section, Schoenwtter bolsters his thesis of environmental changes by brief references to developments and movements of peoples in the areas inhabited by the Anasazi, the Cohonina, the Point of Pines people, and the Sinagua. In this section, he discusses the Athapascanspeaking peoples, their way of life, their aggressiveness, and the effect they might have had on the sedentary Pueblo farmers. He does not say that the Athapascans displaced the Pueblo farmers. He suggests that the Apaches and Navahos might have served as a cultural barrier to the Pueblo peoples if the latter had attempted to move back into their old haunts if and when environmental conditions had improved in their old home lands. This provides a reasonable answer to a question that has always bothered me; namely, why did not the Indians of the Hopi and Zuni refuge areas expand and move back to the Show Low, Snowflake, Springerville, Pine Lawn and Reserve areas? I like this hypothesis and shall use it until it is weakened by new and contradictory data. 


\section{Bibliography}

Agogino, George, and Hester, Jim

1956. Re-evaluation of the San Jose non-ceramic cultures. El Palacio, vol. 63, pp. 6-21.

ANtevs, ERnst

1955. Geologic-climatic dating in the West. American Antiquity, vol. 20, no. 4, pt. 1, pp. 317-335.

Arms, Bernard C.

1960. A silica depressant method for concentrating fossil pollen and spores. Micropaleontology; vol. 6, no. 3, pp. 327-328.

BAKER, O. E.

1936. Atlas of American agriculture. United States Department of Agriculture, Bureau of Agricultural Economics, Government Printing Office, Washington, D.C.

Baldwin, Gordon C.

1939a. Excavations at Kinishba Pueblo, Arizona. American Antiquity, vol. 4, no. 1, pp. 11-21.

1939b. The material culture of Kinishba. American Antiquity, vol. 4, no. 4, pp. 314-327.

Bartlett, Katharine

1933. Pueblo milling stones of the Flagstaff region and their relation to others in the Southwest. Museum of Northern Arizona, Bull. 3.

1934. The material culture of Pueblo II in the San Francisco Mountains, Arizona. Museum of Northern Arizona, Bull. 7.

Beals, Ralph L., Brainerd, George W., and Smith, Watson

1945. Archaeological studies in northeast Arizona. University of California Publications in American Archaeology and Ethnology, vol. 44, no. 1.

Bennett, Mendell C. (Editor)

1948. A reappraisal of Peruvian archacology. Society for American Archaeology, Memoir no. 4 (American Antiquity, vol. 13, no. 4, pt. 2).

Bluhim, Elaine A.

1957. The Sawmill Site. A Reserve Phase village, Pine Lawn Valley, western New Mexico. Fieldiana: Anthr., vol. 47, no. 1.

1960. Mogollon settlement patterns in Pine Lawn Valley, New Mexico. American Antiquity, vol. 25 , pp. 538-546.

Brainerd, George W.

1951. The place of chronological ordering in archaeological analysis. American Antiquity, vol. 16, no. 4, pp. 301-313.

Brand, D. D., Hawley', F. M., and Hibben, F. C.

1937. 'Tseh Tso, a small house ruin, Chaco Canyon, New Mexico. University of Mexico, Anthr. Ser., vol. 2, no. 2 (wholc number 308).

BRETERNITZ, DaVid A.

1957. Additional tool types from Concho. Plateau, vol. 29, no. 4, pp. 78-80. 
1959. Excavations at Nantack Village, Point of Pines, Arizona. University of Arizona, Anthropological Papers, no. 1.

BRYAN, KIRK

1925. Dates of channel trenching (arroyo-cutting) in the arid Southwest. Science, vol. 57 , pp. $339-344$.

Bunzel, Ruth L.

1929. The Pueblo potter: A study in creative imagination in primitive art. Columbia University, Contributions to Anthropology, vol. 8.

1932. Zuni ritual poetry. Bureau of American Ethnology, 47 th Ann. Rept., 192930, pp. 611-835.

Campbell, Elizabeth W. Crozier and William H.

1935. The Pinto Basin Site. An ancient aboriginal camping ground in the California desert. Southwest Museum Papers, no. 9, pp. 21-31.

Carwood, Louis R., and Spicer, Edward H.

1935. Tuzigoot, the excavation and repair of a ruin on the Verde River near Clarkdale, Arizona. National Park Service, Berkeley, California. (Mimeographed.)

Colton, Harold S.

1936. The rise and fall of the prehistoric population of northern Arizona. Science, vol. 84 , pp. 337-343.

1941. Winona and Ridge Ruin. Notes on the technology and taxonomy of the pottery. Museum of Northern Arizona, Bull. 19.

1946. The Sinagua. A summary of the archaeology of the region of Flagstaff, Arizona. Museum of Northern Arizona, Bull. 22.

1949. The prehistoric population of the Flagstaff area. Plateau, vol. 22, no. 2, pp. 21-25.

1959. Hopi kachina dolls with a key to their identification. University of New Mexico Press, Albuquerque.

Colton, H. S., and Hargrave, L. L.

1937. Handbook of northern Arizona pottery wares. Museum of Northern Arizona, Bull. 11.

Cosgrove, H. S. and C. B.

1932. The Swarts Ruin. Papers, Peabody Muscum of American Archaeology and Ethnology, vol. 15, no. 1.

Cummings, BYron

1940. Kinishba, a prehistoric pueblo of the Great Pueblo period. Tucson, Arizona.

Dansereau, Pierre M.

1957. Biogeography: an ecological perspective. Ronald Press, New York.

Danson, EDWARd B.

1957. An archaeological survey of west central New Mexico and cast central Arizona. Papers, Peabody Museum of American Archaeology and Ethnology, vol. 44 , no. 1 .

Danson, E. B., and Malde, H. E.

1950. Casa Malpais, a fortified pueblo site at Springerville, Arizona. Plateau, vol. 22, no. 4, pp. 61-67.

Di Peso, Citarles C.

1950. Painted stone slabs of Point of Pines, Arizona. Amcrican Antiquity, vol. 16, no. 1 , pp. 57-65.

1951. The Babocomari village site on the Babocomari River, southeastern Arizona. The Amerind Foundation, no. 5. 
1953. 'The Sobaipuri Indians of the upper San Pedro River Valley, southeastern Arizona. The Amerind Foundation, no. 6.

1956. The upper Pima of San Cayetano del Tumacacori, an archaeohistorical reconstruction of the Ootam of Pimeria Alta. The Amerind Foundation, no. 7.

1958. The Reeve Ruin of southeastern Arizona. A study of a prehistoric Western Pueblo migration into the middle San Pedro Valley. The Amerind Foundation, no. 8 .

Erdtman, Gunnar

1954. An introduction to pollen analysis. Chronica Botanica Company, Waltham, Massachusetts.

Faegri, Knut, and Iverson, Joliannes

1950. Textbook of modern pollen analysis. E. Munksgaard, Copenhagen.

Fewkes, J. Walter

1894. Dolls of the Tusayan Indians. Internationales Archiv für Ethnographie, Band 7, pp. 45-73. Leiden.

1903. Hopi katcinas drawn by native artists. Bureau of American Ethnology, 21st Ann. Rept., 1899-1900, pp. 3-126, pls. 2-63.

1904. Two summers' work in Pucblo ruins. Bureau of American Ethnology, 22nd Ann. Rept., 1900-1901, pp. 1-197.

1924. The use of idols in Hopi worship. Smithsonian Institution, Ann. Rept. for 1922, pp. 377-397.

Fewkes, J. Walter, and Stephen, Alexander M.

1892. The Na-ac-nai-ya: a Tusayan initiation ceremony. Journal of American Folklore, vol. 5, no. 18, pp. 189-217.

Frey, David C.

1935. A differential flotation technique for recovering microfossils from inorganic sediments. New Phytologist, vol. 54, no. 2, pp. 257-258.

Gladwin, Harold S.

1945. The Chaco Branch. Excavations at White Mound and in the Red Mesa Valley. Gila Pueblo, Medallion Papers, no. 33. Globe, Arizona.

Gladwin, H. S., Haury, E. WV., Say'les, E. B., and Gladwin, N.

1937. Excavations at Snaketown. Gila Pueblo, Medallion Papers, no. 25. Globe, Arizona.

GladWin, Winifred and Harold S.

1931. Some southwestern pottery types. Series II. Gila Pueblo, Medallion Papers, no. 10. Globe, Arizona.

1934. A method for the designation of cultures and their variations. Gila Pueblo, Medallion Papers, no. 15. Globe, Arizona.

Hastings, James R.

1960. Vegetation change and arroyo cutting in southeastern Arizona. Journal of Arizona Academy of Science, vol. 1, no. 2, pp. 60-67.

HAURY, EMIL IV.

1934. The Canyon Creek Ruin and the cliff dwellings of the Sierra Ancha. Gila Pueblo, Medallion Papers, no. 14. Globe, Arizona.

1936a. The Mogollon culture of southwestern New Mexico. Gila Pueblo, Medallion Papers, no. 20. Globe, Arizona.

1936b. Some southwestern pottery types. Series IV. Gila Pueblo, Medallion Papers, no. 19. Globe, Arizona.

1940. Lxcavations in the Forestdale Valley, east-central Arizona. University of Arizona Bull., vol. 11, no. 4 (Social Science Bull., no. 12). 
1945. The excavations of Los Muertos and neighboring ruins of the Salt River Valley, southern Arizona. Papers, Peabody Museum of American Archaeology and Ethnology, vol. 24, no. 1.

1950. The stratigraphy and arehacology of Ventana Cave, Arizona. University of Arizona Press, Tucson.

1957. An alluvial site on the San Carlos Indian Reservation, Arizona. American Antiquity, vol. 23 , no. 1 , pp. 2-27.

HAUR', EMIL W', and Hargrave, LYNdon L.

1931. Recently dated pueblo ruins in Arizona. Smithsonian Miscellaneous Collections, vol. 82, no. 11.

Hauri, Emir. W., and Say'les, E. B.

1947. An early pit house village of the Mogollon culture, Forestdale Valley, Arizona. University of Arizona Bull., vol. 18, no. 4 (Social Science Bull., no. 16).

Hodge, F. W.

1920. Hawikuh bonework. Museum of the American Indian, Heye Foundation, Indian Notes and Monographs, vol. 3, no. 3.

1922. Recent excavations at Hawikuh. El Palacio, vol. 12, no. 1, pp. 1-11.

1923. Circular kivas near Hawikuh, New Mexico. Contributions from the Museum of the American Indian, Heye Foundation, vol. 7, no. 1.

1939. A square kiva near Hawikuh. In "So live the works of men," Seventieth Anniversary Volume honoring Edgar L. Hewett. University of New Mexico Press.

HoIJER, HARry

1956. The chronology of the Athapascan languages. International Journal of American Linguistics, vol. 22, no. 4, pp. 219-232.

\section{Hovgh, Walter}

1907. Antiquities of the upper Gila and Salt River Valleys in Arizona and New Mexico. Bureau of American Ethnology, Bull. 35.

1914. Culture of the ancient pueblos of the upper Gila River region, New Mexico and Arizona. United States National Museum, Bull. 87.

1919. The Hopi Indian collection in the United States National Museum. Proceedings, United States National Museum, vol. 54, no. 2235, pp. 235-296.

JELINEK, ARTIIUR J.

1960. An archaeological survey of the Middle Pecos River Valley and the adjacent Llano Estacado. University of Michigan (microfilms).

Jexinings, Jesse D.

1957. Danger Cave. Memoirs, Socicty for American Archaeology, no. 14.

Jennings, Jesse D., and Reed, Erik

1956. The American Southwest, a problem in cultural isolation. Memoirs, Society for American Archaeology, no. 11 (American Antiquity, vol. 22, no. 2, pt. 2 , pp. 58-127).

JUdD, NeIL $M 1$.

1954. The material culture of Pueblo Bonito. Smithsonian Miscellaneous Collections, vol. 124.

Kext, Kate Peck

1957. The cultivation and weaving of cotton in the prehistoric southwestern United States. Transactions of the American Philosophical Society, new ser., vol. 47, pt. 3.

KIDDER, A. V.

1932. The artifacts of Pecos. Papers of the Southwestern Expedition, no. 6. Phillips Academy, Andover, $\mathrm{N}$ assachusetts. 
1958. Pecos, New Mexico: Archacological notes. Papers of the Robert S. Peabody Foundation for Archacology, vol. 5. Phillips Academy, Andover, Massachusetts.

Lamblet, Marjorie F.

1957. A rare stone humplacked figurine from Pecos Pucblo, New Mexico. El Palacio, vol. 64, nos. 3-4, pp. 93-107.

LEMMTR, DONALD J.

1951. Robinson's coeflicient of agreement - a critique. American Antiquity, vol. 17 , no. 2 , p. 151 .

Martin, Paul S.

1936. Lowry Ruin in soutlwestern Colorado. Ficld Mluscum of Natural History, Anthr. Ser., vol. 23, no. 1.

1939. Modified Basket Maker sites in the Ackmen-Lowry area, southwestern Colorado, 1938. Field Museum of Natural History, Anthr. Ser., vol. 23, no. 3.

1943. The SU Site. Excavations at a Mogollon village, western New Mcxico, 1941. Field Museum of Natural History, Anthr. Ser., vol. 32, no. 2.

Martin, Paul S., and Rinaldo, John B.

1940. The SU Site. Excavations at a Mogollon village, western New Mexico, 1939. Field Museum of Natural History, Anthr. Ser., vol. 32, no. 1.

1947. The SU Site. Excavations at a Mogollon village, western New Mexico, 1946. Field Museum of Natural History, Anthr. Ser., vol. 32, no. 3.

1950a. Turkey Foot Ridge Site. A Mogollon village, Pinc Lawn Valley, western New Mexico. Fieldiana: Anthr., vol. 38, no. 2.

1950b. Sites of the Reserve Phase, Pine Lawn Valley, western New Mexico. Fieldiana: Anthr., vol. 38, no. 3.

1960a. Excavations in the Upper Little Colorado drainage, eastern Arizona. Fieldiana: Anthr., vol. 51, no. 1.

1960b. Table Rock Pueblo, Arizona. Fieldiana: Anthr., vol. 51, no. 2.

Martin, P. S., Rinaldo, J. B., and Antevs, Ernst

1949. Cochise and Mogollon sites, Pine Lawn Valley, western New Mexico. Fieldiana: Inthr., vol. 38, no. 1.

Martin, P. S., Rinaldo. J. B., and Barter, Eloise R.

1957. Late Mogollon communities. Four sites of the Fularosa Phase, western New Mexico. Fieldiana: Anthr., vol. 49, no. 1.

Martin, P. S., Rinaldo, J. B., and Bluhim, Elaine

1954. Caves of the Reserve area. Fieldiana: Anthr., vol. 42.

Martin, P. S., Rinaldo, J. B., Bluhim, E., and Cutler, H. C.

1956. Higgins Flat Pueblo, western New Mexico. Fieldiana: Anthr., vol. 45.

Martin, P. S., Rinaldo, J. B., Bluhi, E., Cutler, H. C., and Grange, Roger, Jr. 1952. Mogollon cultural continuity and change. The stratigraphic analysis of Tularosa and Cordova caves. Ficldiana: Anthr., vol. 40.

Martin, P. S., Rinalido, J. B., and Longagre, W. A.

1960. Documentation for some late Mogollon sites in the upper Little Colorado drainage, castern Arizona. Archives of Archacology, no. 6 (3 microcards), Society for American Archaeology and the University of Wisconsin Press, Madison.

1961. Mineral Crcek Site and Hooper Ranch Pueblo, eastern Arizona. Fieldiana: Anthr, vol. 52.

Martin, P. S., Rinatido, J. B., Longacre, W. A., and Freeman, Leslie G., Jr.

1961. Documentation for prehistoric investigations in the upper Little Colorado drainage, eastern Arizona. Archives of Archacology, no. 13 (3 microcards), Society for American Archacology and the University of Wisconsin Press, Madison. 
Martin, P. S., and Wililis, E. S.

1940. Anasazi painted pottery in Field Museum of Natural History. Field Museum of Natural History, Anthr. Mem., vol. 5.

Martin, Paul S., ${ }^{1}$ and Schoenivetter, James

Manuscript a. Pollen analysis of alluvium from Binne-Ettini Canyon. University of Arizona.

Manuscript b. Pollen stratigraphy of a great kiva from Chaco Canyon. University of Arizona.

1960. Arizona's oldest cornfield. Science, vol. 132, no. 3418, pp. 33-35.

Martin, Paul S., 1 Schoenwetter, James, and Aris, B. C.

1961. Southwestern palynology and prehistory: the last 10,000 years. Contribution no. 50, Program in Geochronology, University of Arizona.

McGregor, Jolin C.

1941. Winona and Ridge ruin. Part 1. Architecture and material culture. Museum of Northern Arizona, Bull. 18.

MiNdelefF, Victor

1891. A study of Pueblo architecture, Tusayan and Cibola. Burcau of American Ethnology, 8th Ann. Rept., 1886-87, pp. 13-228.

Morris, Earl H.

1919. The Aztec Ruin. American Museum of Natural History, Anthropological Papers, vol. 26, pt. 1.

1921. The house of the Great Kiva at the Aztec Ruin. American Museum of Natural History, Anthropological Papers, vol. 26, pt. 2.

Morris, E. H., and Burgit, Robert F.

1954. Basket Maker II sites near Durango, Colorado. Carnegic Institution of Washington, Publ. 604.

Nesbitt, Paul H.

1931. The ancient Mimbrenos, based on investigations of the Mattocks Ruin, Mimbres Valley, New Mexico. Logan Museum Publications in Anthropology, Bull. no. 4, Beloit College, Beloit, Wisconsin.

1938. Starkweather Ruin. Logan Museum Publications in Anthropology, Bull. no. 6, Beloit College, Beloit, Wisconsin.

Nichol, A. A.

1952. The natural vegetation of Arizona. University of Arizona Agricultural Experimental Station, Bull. 127.

Olson, Alan P.

1960. The Dry Prong Site, east central Arizona. Contributions to Point of Pines Archaeology, no. 15 (American Antiquity, vol. 26, no. 2, pp. 185-204).

Opler, Morris E.

1941. An Apache life-way: the economic, social, and religious institutions of the Chiricahua Indians. University of Chicago Press.

Parsons, Elsie C. (Editor)

1936. Hopi journal of Alexander M. Stephen. 2 vols. Columbia University Contributions to Anthropology, no. 23.

Parsons, Elsie C.

1939. Pucblo Indian religion. 2 vols. University of Chicago Press.

${ }^{1}$ Dr. Paul S. Martin of Geochronology Laboratories, The University of Arizona, Tucson. 
Peckham, Stewart

1958. Hillside Pueblo: early masonry architecture in the Reserve area, New Mexico. El Palacio, vol. 65, no. 3, pp. 81-94.

Peppler, George: H.

1920. Pueblo Bonito. American Museum of Natural History, Anthropological Papers, vol. 27.

Rands, RoBert L.

1961. Elaboration and invention in ceramic traditions. American Antiquity, vol. 26, no. 3, pt. 1, pp. 331-340.

REED, ERIK K.

1955. Painted pottery and Zuni history. Southwestern Journal of Anthropology, vol. 11, no. 2, pp. 178-193.

Rilli:, Carroll L.

1954. A survey of Navaho archaeology. University of Colorado Studies, Series in Anthropology, no. 4.

Rinaldo, Join B.

1959. Foote Canyon Pueblo, eastern Arizona. Fieldiana: Anthr., vol, 49, no. 2.

Rinaldo, J. B., and Bluim, E. A.

1956. Late Mogollon pottery types of the Reserve area. Fieldiana: Anthr., vol. 36, no. 7 , pp. 149-187.

Robbins, Wilfred Willial, Harrington, John Peabody, and Freire-Marreco, BARBARA

1916. Ethnobotany of the Tewa Indians. Bureau of American Ethnology, Bull. 55.

Roberts, F. H. H., JR.

1931. Ruins at Kiatuthlanna, eastern Arizona. Bureau of American Ethnology, Bull. 100.

1932. The Village of the Great Kivas on the Zuni Reservation, New Mexico. Bureau of American Ethnology, Bull. 111.

1939. Archaeological remains in the Whitewater district, eastern Arizona. Part I : House types. Bureau of American Ethnology, Bull. 121.

1940. Archaeological remains in the Whitewater district, eastern Arizona. Part II : Artifacts and burials. Bureau of American Ethnology, Bull. 126.

Robinson, W. S.

1951. A method for chronologically ordering archaeological deposits. American Antiquity, vol. 16, no. 4, pp. 293-301.

Rowe, Chander

1947. The Wheatley Ridge Site. Unpublished thesis for degree of Master of Arts, University of Chicago.

SAPIR, EdWARD

1921. Language. Harcourt, Brace \& Co., New York.

SAyles, E. B.

1945. The San Simon Branch. Excavations at Cave Creek and in the San Simon Valley. I. Material culture. Gila Pueblo, Medallion Papers, no. 34. Globe, Arizona.

Saýles, E. B., and Antevs, Ernst

1941. The Cochise culture. Gila Pueblo, Medallion Papers, no. 29. Globe, Arizona. 
SChoenwetTer, James

1960a. Pollen analysis of sediments from Matty Wash. Thesis for degree of Master of Arts, Department of Botany, University of Arizona.

1960b. Pollen stratigraphy of the Wetherill Mesa region. MIS. Report to the National Park Service.

Schroeder, A. H.

1948. Montezuma well. Plateau, vol. 20, no. 3, pp. 37-40.

1960. The Hohokam, Sinagua and the Hakataya. Archives of Archaeology, no. 5

(4 microcards), Society for American Archaeology and the University of Wisconsin Press, Madison.

Schulmax, Edmond

1956. Dendroclimatic changes in semiarid America. University of Arizona Press, Tucson.

Schwartz, Douglas W.

1956. The Havasupai 600 A.D.-1955 A.D.: a short culture history. Plateau, vol. 28, no. 4 , pp. $77-85$.

1957. Climatic change and culture history in the Grand Canyon region. American Antiquity, vol. 22 , no. 4 , pp. 372-377.

Smiley, Terah L.

1952. Four late prehistoric kivas at Point of Pines, Arizona. University of Arizona Bull., vol. 23, no. 3 (Social Science Bull., no. 21).

Sмiтн, H. V.

1956. The climate of Arizona. University of Arizona Agricultural Experimental Station, Bull. 279.

Smith, Watson

1950. Preliminary report of the Peabody Museum Upper Gila Expedition, Pueblo Division. EI Palacio, vol. 57, no. 12, pp. 392-399.

1952a. Excavations in Big Hawk Valley, Wupatki National Monument, Arizona. Museum of Northern Arizona, Bull. 24.

1952b. Kiva mural decorations at Awatovi and Kawaika-a, with a survey of other wall paintings in the Pueblo Southwest. Papers, Peabody Museum of American Archaeology and Ethnology, vol. 37.

Spier, LesLie

1917. An outline for a chronology of Zuni ruins. American Museum of Natural History, Anthropological Papers, vol. 18, pt. 3.

1918. Notes on some Little Colorado ruins. American Museum of Natural History, Anthropological Papers, vol. 18, pt. 4.

Stephen, Alexander M.

1936. See Parsons, Elsie C. (Editor)

Stevenson, Matilda C.

1904. The Zuni Indians; their mythology, esoteric fraternities, and ceremonies.

Bureau of Amcrican Ethnology, 23rd Ann. Rept., 1901-02, pp. 13-604.

Stewart, G. R., and Donnelly, Maurice

1943. Soil and water economy in the Pueblo Southwest: I, Field studies at Mesa Verde and northern Arizona; II, Evaluation of primitive methods of conservation.

Scientific Monthly, vol. 56, nos. 1 and 2, pp. 31-44, 135-144.

Thomas, Tully H.

1953. The Concho complex: a popular report. Plateau, vol. 25, pp. 1-10. 
Titrev, Miscui

1944. Olel Oraibi, a study of the Hopi Indians of Thirel Mesa. Papers, Peabody Museum of American Archacology and Ethnology, vol. 22, no. 1.

Trewartiha, Grenn 'T.

1954. An introduction to climate. McGraw-Hill Book Co., New York.

Vivian, Gordon, and Reiter, Paul

1960. The Great Kivas of Chaco Canyon and their relationships. Monographs of the School of American Research and the Museum of New Mexico, no. 22. Santa Fe.

V'OTH, H. R.

1901. The Orailsi Powamu ceremony. Field Museum of Natural History, Anthr. Ser., vol. 3, no. 2, pp. 61-158.

1912. 'The Oraibi Marau ceremony. Field Museum of Natural History, Anthr. Ser., vol. 11, no. 1.

WENDORF, Fred

1950. A report on the excavation of a small ruin near Point of Pines, east central Arizona. University of Arizona Bull., vol. 21, no. 3 (Social Science Bull., no. 19).

1953. Archaeological studies in the Petrified Forest National Monument. Museum of Northern Arizona, Bull. 27.

Wendorf, Fred, and Thomas, Tully $\mathrm{H}$.

1951. Early Man sites near Concho, Arizona. American Antiquity, vol. 17, pp. 107-114.

WheAt, Joe B.

1954. Crooked Ridge Village (Arizona W:10:15). University of Arizona Bull., vol. 25 , no. 3 (Social Science Bull., no. 24).

WHITING, A. F.

1939. Ethnobotany of the Hopi. Museum of Northern Arizona, Bull. 15.

Willey, Gordon R.

1945. Horizon styles and pottery traditions in Peruvian archacology. American Antiquity, vol. 12, pp. 132-134.

1948. Functional analysis of "horizon styles" in Peruvian archaeology. In BENNETT, WENDELL C. (Editor), 1948.

WODEHOUSE, R. D.

1935. Pollen grains. McGraw-Hill Book Co., New York.

WOONBURY, RiCIIARD

1954. Prehistoric stone implements of northeastern Arizona. Papers, Peabody Museum of American Archacology and Ethnology, vol. 34 (Reports of the Awatovi Expedition, no. 6).

1961. Prehistoric agriculture at Point of Pines, Arizona. Memoirs, Society for American Archacology, no. 17 (American Antiquity, vol. 26, no. 3, pt. 2).

Woobbury, Riciiard B., and Nathalie, F. S.

1956. Zuni prehistory and El Morro National Monument. Southwestern Lore, vol. 21 , no. 4 , pp. 56-60.

Wyman, Leland C.

1952. The sand paintings of the Kayenta Navaho. University of New Mexico Publications in Anthropology, no. 7. 


\section{Index}

Abajo Red-on-Orange, 105

Abrading stones, 135; grooved, 132

Acoma kivas, 66

Adobe, 28; calking, 214; clay, 48, 54; plaster, 46, 62

Agogino, George, 155

Agriculturalists, 201, 204, 205, 206

Agriculture, 200, 215, 217; beginnings of, 164, 165: dry farming, 202; economy, 196; expanding, 166; Mogollon area, 191; see also Irrigation

Alder, 174

Alma Plain, 150, 159

Almus, 173,174

Alschuler, William, 4

Altars, Hopi (Marau, Wuwutcim, Talatumsi and Marau-mana ceremonies), 72 Amaranthus, 174, 186, 200

Amargosa II points, 155

Anasazi, 160, 166, 167, 227: Chacoan, 214: culture, 63, 144, 201, 202: Great Kivas, $60,66,67,68,214$ : irrigation system, 203; lesser kivas, 65, 66, 68: tradition, 64, 66; traits, 163; see also Pueblo III sites

"Antechamber," 36, 37

Antevs, Ernst, 122, 196

Anthropomorphic figure; see Image

Apaches, 224, 227; Western, 205

Arboreal plants, 173: pollen, 179, 181 $183,185,186,188,189,191,194,195$ 196,206

Archacological Reconnaissance, 224; research, 4; survey area 149; survey program, 222

Architectural, development, Mogollon, 221; traditions, 69

Arizona W : $10: 51,144,145$

Arizona $\mathrm{W}: 10: 52,60,124$

Armillas, Prof. P., 168

Arms, Bernard C., 171, 177, 185, 197

Arrow-shaft, smoother, 132; straightener, 132; tools, 132, 135, 147

Arroyo-cutting hypothesis, 196, 197, 198

Arroyo bank, 206

Arroyo sites, 177

Artifacts, 115-147; stone, 210, 211

Ash pits, 63, 66

Athapascan-speakers, 205, 206, 227

Awatovi, 66; murals, 73, 74
Awls, bone, 139, 141; bone splinters, 139; split long-bone type, 139; ulna type, 139,147

Awl-sharpening stone, 48

Axe-grinding slab, 129, 131

Axes, stone, $115,127,129,130 ; \mathrm{full}$ grooved, 115; three-quarter grooved, 129, 147; tabular. 129

Axis, secondary, of kiva, 66

Aztec Ruin, 145

Babocomari Village, 140

Baker, O. E., 201

Baldwin, Gordon C., 145

Banquettes, 66

Barreras, Wilfred, 4

Bartlett, Katherine, 146

Basalt, boulders, 20, 32, 45, and cobbles, 23,36

Basket Maker III sites, 60

Beach sites, 116, 122, 155, 156, 163, 164, 178

Beads, 58, 71.214; olivella, truncate, 144; stone and shell, 140

Beals, Ralph L., 105

Beams, 50, 59, 64, 65; main, 37

Beans, 68

Bench, 51, 54, 58, 60, 64. 66, 68, 213

Bin, 55, 63; corner, 47, 48

Binne-Ettini Canyon, 174

Black Mesa Black-on-White, 105, 106

Blades, 130, 132, 134, 138; fragments, 158

Blue River, 60, 64

Bluff Site, 61, 116

Bluhm, Elaine A., 64, 106, 164; see also Sawmill Site

Bodkins, 139, 140: tip, 141

Bone, awls, 139; effigy pendant, 140; fragment, incised, 141

Boreal economy, 205, 206

Boyer, Walter, 5, 106, 223

Bracelets, shell, 140, 144; fragments, 143

Brainerd, George W., 87, 105, 107

Brainerd-Robinson, methorl, 107; ratios, 114; see also Robinson-Brainerd technique

Brand, D. D., 126

Brawley, Elizabeth, 5

Breternitz, David A., 60, 62, 63, 64, 124, $125,132,138,139,157$ 
Brinkerhoff, Mr. and Mrs. Wayne, 5

Brown indented corrugated, 79

Bryan, Kirk, 205

Bunzel, Ruth L., 69, 71, 77

Buttress, 51

Cactaceac, 174

Cactus family, 174

Cahonc Canyon sites, 60

Canyon Creck Ruin, 129, 145

Carbon-14 dating, 211, 221; sce also Charcoal

Carter, Mr. and Mrs. J. R., 5

Casa Malpais, 60

Cattail, 177, 195; pollen of, 221

Caywood, Louis R., 140, 146

Ciedar, 177

Ceiling, 50; sec also Roof

Ceramics, 68; materials, 76; traditions, 69: sec also Chacoan ceramics

"Ceramic Group," 150; see also Preceramic site

Ceremonial, objects, 145; use, Room C, Rim Valley, 51, 53, 220; room, 217

Ceremonies, group, 220

Ceremony, contemporary, 68: hypothetical, 68

Chaco Canyon, 50, 174; Great Kivas, 66

Chaco district, 115 ; tradition of Anasazi culture, 63, 68

Chacoan Anasazi, 214

Chacoan ceramics, early, 223, 224

Charcoal, 178, 211, 215

Chase, Ellen, 4

Cheno-am, 178, 179, 180, 181, 183, 185, 186, 188-191, 194-198, 206; definition of, 174

Chenopodiaceae, 174, 186

Chilcott, D., 4

Chilcott Site 1, 30, 31, 32-34, 36-37, 62, $78,95,107,108,109,118,119,131$, $136,137,138,140,142,145,146,216$, 217

Chilcott Site 2, 35, 37, 95, 107, 109, 146 , 216

Chilcott Site 3, 35-38, 62, 95, 216

Chilcott Sites, 3, 29-38, 61, 116, 119, 124 , 218; intra-site seriation, 95 ; pottery of, 77, 80, 102; settlement pattern, 216; summary, 212; totals of sherds, 82; trends in painted pottery types, 97

Childbirth water house, Tihkuyiki, 67

Chinking, 44, 54

Chiricahua Apache, 205

Chiricahua Stage, Cochise culture, 122

Choppers, 129, 130, 131, 147, 161, 162; biface type, 130; included with axes, 130; uniface type, 130

Cienaga Sitc, 116

Cienaga soils, 197

Circle Prairie Phase, 64, 132
Cleome, 172, 174, 182, 183, 184; serrulata, 174

Climatic changes, 191-194

Climate, clues to, 224; shift in micro-, 226

Cochise, Wet Leggett, 61; culture, 116 , 122,177 ; industries, 156

Coefficients of similarity, 90, 107, 108

Cohonina, population movements, 203, 227

Collecting scason, 163

Colorado Plateau, 148

Colton, H. S., 71, 105, 150, 204

Compositac, 174, 178-181, 183, 185, 186, $188,191,194-198$

Concho, Arizona, 29, 138

Concho Complex, 156, 157, 165

Construction of house, tools for, 128

Contamination of soil samples, 171

Cooking, 215, 220, 221

Corn, 68, 165, 177, 215, 216; growing season, 201 ; pollen, 215,221

Cosgrove, H. S. and C. B., 72

Cotton, 177

Counting and identification of pollen, 172

Cox, Mr. and Mrs. Tom, 6

Crooked Ridge Village, 121

Crushing tools, 120

Crypt, 57-58, 59, 64, 67, 71, 73, 74, 214; wall, 66; double cover, 67; meaning of aperture, 73

Cucurbita, 174, 182, 183

Cult deity, 71, 74; Alosaka, 72; female character of, 73: Hopi figurines, 73; relation to underworld, 73, 74; Tuwapongtumsi, 73; Talatumsi, 73; concerned with childbirth, 73

Cultigens, 173, 183, 184, 200

Cultural influences (pottery), 103

Cultural lag, 115

Culture and environment, relationship between, 169

Cummings, Byron, 73, 218, 220

Curb (or lip), 20, 24

Cylinder stones, 145, 147

Cyperaceae, 173, 174, 183, 185, 190, 195. 196

D-shaped pithouses, 26

"Damper," 36: slab, 49

Danger Cave, 156

Danson, E. B., 60, 164, 212

Defense systems, 210

Deflector, $34,56,57,58,64,65,68,115$, 213

Deity, cult, 71 ; see also Cult deity

Descrt Culture, 155, 156, 163, 164, 165

Design elements, analysis of, 105-114, 223; pottery, 75, 105, 163, sorted by, 106, 107; at given sites, 112-113; names and code numbers of, 110-111; trends in, 109 
Dimensions of rooms, 32, 43

Di Peso, Charles C., 140, 144, 145

Directional colors, 69

Dockstader, Dr. Frederick J., 6

Dolls, 74; older flat type, 72

Donnelly, Maurice, 203

Doors (at Kinticl), 67

Doorways, 46

Drainages, clues to, 224

Drift, general, in pottery design changes, 77,108 ; linguistic, 108

Drills, 135, 138, 139, 147; in very early levels, 139

Dry farming, 202, 204

Dry Prong Site, Great Kiva, 65, 66, 68

Dwelling rooms, 53

Economic plants, 173, 181, 182, 185, 190

Economy, "Tumbleweed Canyon Site, 215; Chilcott Sites, 217: Thode Site, 217-218; Rim Valley Pueblo, 220; Hooper Ranch Pueblo, 221

Effigy pendant, bone, 140

Effigy; see Image

Eggan, Dr. Fred, 6, 74

Elden Pueblo, 145

Entrance, 20, 25, 28, 66; spirit's, 67; type of, 64,65

Entryways, lateral, 65, 67, 68; ramp, 57 , 58,213

Environmental, change, definition of, 194; conditions, pre-existing, 194; periods, 190, 191; shifts, 202, 203, 204, 225, 227

Environments, prehistoric, 198-206

Ephedra, 174. 185

Erdtman, Gunnar, 171

Erosion controls, 204

Exogamous clans, 217, 218

Extended families, 218

Extraction techniques (pollen analysis), 171

Facgri, Knut, 171

Female symbol, yellow, 22

Fennell, Agnes McNary, 6

Fewkes, J. Walter, 67, 71, 72, 73, 146

Field, President Stanley, 6

Figurine; sec Image, 214

Fireboxes, 62, 63

Firepits, 19, 20, 22, 24, 25, 28, 34, 37, 38, $40,46,48,49,51,55,57,62,66,68$, $126,220,221$; area, 65 ; circular, 60 , 62; small, 68

Fireplace, 60

Fire screen, 68

Flagstaff area, 140, 145, 146, 204

Flakes, utilized, 135

Flattop Site, 157

Floor plan, shape of, 64,68

Floors, 19, 22, 23, 28, 32, 33, 37, 40, 48, 54,61

Flour receptacles, 47, 48, 63, 122
Food preparation, tools, 115-126

Foot drums, 68; see also Vaults

Foote Canyon Pueblo, 126, 129, 218; plaza. 65

Forest, 174, 188, 189, 191, 195, 200, 205, 206; re-growth, 206

Forestdale Phase, 138

Forestdale Site, 116, 138

Forslev, Dr. Albert, 6

Four Mile Polychrome, 214; bowls with anthropomorphic figures, 73

Four Mile Ruin, 67

Frazier, V'ernon, 5

Furnishings, interior, 62

Geochronology Laboratories, 168, 171

Gila River, upper, 134

Gillespie, Mr. and Mrs. Charles, 6

Gillespie, Mr. and Mrs. Leon, 6

Gillespie, Mr. and Mrs. Milton, 6

Girders, 37, 65; see also Beams

Gladwin, Harold S., 65, 105, 140, 144, 145

Globe mallow, 177

Goesling, AI. H., 4

Goesling Site, 3, 26-29, 131, 137-143, 146, 223; intra-site seriation, 94; potterv of, 77, 78, 80,102, 107, 108, 109, $120,122,125,128$, types by levels, 92, totals of sherds, 81 ; settlement pattern, 216: summary, 212

Goodman, Mr. and Mrs. Donald, 6

Goodman, Joe, 4

Goosefoot family, 174

Gramineae, $177^{\circ}$

Grand Canyon area, 203, 204

Grass family, 177

Grasslands, 173, 188, 196

Great Kiva, Higgins Flat, early, 65 ; Hooper Ranch Pueblo, 3, 52, 53-60, $64-68,115$, pottery of, 77,80 , totals of sherds, 85-86, stone discs, 125, west wall, 71; see also Hooper Ranch Pueblo

Great Kivas, 161, 162; comparisons of, 60; Mogollon, 67: Village of the, 60

Gregg, Dr. Clifford C., 7

Grinding stones, small metate-like, 124

Groningen Laboratory, 178, 211, 212, 215,221

Gurley, C. E., 6

Haas, Dr. Fritz, 6

Hahn, Mr. and Mrs. Maxwell, 6

Hammer, grooved, 129; see also Maul

Hammerstones, 48, 126

Hargrave, I.. L., 145

Harvest cycle, 163

Harvey, Byron, III, 6

Hastings, James R., 205

Hatchways (at Kintiel), 67

Haury, Emil W., 61, 64, 65. 76, 116, 122, $129,130,138,140,145,155,178,212$ 
Hawikuh, 65, 145

Hawley, 1 . M1., 126

Hearth, 68; area, 65, 66; raised, 60

Herod, David, 4

Heshota-uthla Polychrome, 80, 214

IHester, E. D., 155

Hibben, F. C., 126

Higgins Flat Pucblo, 60, 65, 124, 181, 186; Great Kiva, 65, 68, 169, 185, 188, 190, 191

Hilitop Phase, 61, 122

Hodge, F. W., 65, 145

Hohokain, 65, 145

Hoijer, Harry, 205

Hooper Ranch PucbIo, 3, 52, 60, 63, 103, $119,120,125-131,134-144,146,182$, 184-190, 219, 221; intra-site seriation, 99; trends in painted pottery types, 101; Great Kiva, 65-68, 125, 132, 136, 138 . $140,145,182,183,187$; settlement pattern, 220-222; summary, 213-215

Hooper, Rob., 4

Hopi,culture, 167; Indians, 227; cult deity figurines, contemporary, 73; kivas, lesser, 66; similarities, image, 69, 71; towns, 222

Horizon markers (pottery), 88

"Horizon styles" (pottery), 75

Houck Polychrome, 80, 213

Hough, Walter, 60, 64, 130

Household utility tools, 135-139

Houses; see Construction of Houses, tools for

Human effigy; see Image

Hunting, and gathering, 198, 200, 201, 215; and warfare, tools, 130-139

Hvgric plants, 173, 181, 182, 183, 185 , 190,195

Image, stone, 57-58, 59, 67, 69-74, 145146, 214: description of, 69; female character, 72

Interdisciplinary co-operation, 225

Irrigation, 201, 202, 203, 205

Iverson, Johannes, 171

Jacal construction, 62

Jar, miniature; see Miniature jar

Jeddito area, 119, 146

Jelinek, Arthur J., 90, 106

Jennings, Jesse D., 155, 156, 201, 203

Jewett Gap Pueblo, 218

Judd, Neil M., 115, 132, 146

Fuglans, 173, 177, 184

Juniper, 177, 179

Juniperus, 173, 177, 178, 179, 180

Kachina-kihu, 66

"Kachina" niche, 51

Kachina, proto $\cdot 71,73,74$

Kachinas, mother of, 67; Citulilu, 72; Patun (Squash), 72; Rainbow, 72
Kana-a Black-on-White, 105, 106

Kayenta pottery types, early, 223, 224

Keney, Dr. Charles $\mathbf{1}$., 6

Kent, Kate Peck, 139

Kiatuthlanna Black-on-White, 102, 105 $109,150,212,213,223$; design elements, 109

Kidder, A. V., 66, 119, 146

Kinishba, 60, 73, 144, 218, 220

Kinticl, 67

Kittle, Mr. and Mrs. Jake, 5

Kiva, 51, 220; -building, 219; entrance, 73; lesser, Anasazi, 65; /uni, 66

Kivas, Anasazi type, 51, 60; circular, 162 , 163; Western Pucblo type, 53. 60, 64

Knives, 132; flake, 135, 137, 147, 158

Kwakina Polychrome, 85,214

Ladder pits(?), 48, 49, 51, 62, 63, 220

Laguna Salada, 61, 155, 178, 186, 191, 198

Lambert, Marjorie F., 6, 146

Lane, Gardner, 4

La Plata Black-on-Orange, 105

L.chmer, Donald J., 87

Leverton, Mr. and Mrs. John D., 5

Lexico-statistics, 205

Lino Gray, 150, 159

Lintel, 36, 46

Little Colorado River, drainage, 50, 51 , $105,145,147$; upper. $63,115,120,130$, 157, 184; Valley, 19, 26, 40, 53, 115 , $134,148,151,161,162,164,182,210$. $219,221,224$

Little Ortega Lake, 116, 155, 178, 186 , 191. 198

Los Muertos Site, 140, 145

Lyman Dam. 210

Lyman Reservoir, 19

Malde, H. E., 60

Mallow family, 177

Malpais rocks, 22

Malvaceae, 177

Manos, 20, 48, 116-119, 122, 126, 147; beveled, 147, 177; grinding surface, 119; flat-tabular, 117; loaf-shaped, 117; on earlier sites, 116; one-hand, 116 . $117,118,146 ;$ two-hand, 116, 117, 147

Marau ceremony, 72

Marau-mana ceremony, 72

Martin, Paul S. (Arizona), 4, 168, 174. $177,185,197,204$

Masauwu, 67

Mask, 71

Masonry, 32, 43, 50, 56, 67, 68, 210, 212; banded, 50, 115; composite construction, 33: jacal construction, 62; rubble, 61 , crude, 36,61 , random type, 32,37 , 44,54 , regularly coursed, 54 ; through stones, 33, 40, 43; Type I, 44, 45, 46; Type II, 44, 45; venecr, 59, 66, 68; vertical slab, 44, 54, 60 
Matrilineal descent, 217, 218, 222

Matrilocal families, 222; residence, 217 , 218

Maul, 127, 129, 147; full grooved, 129; tabular, 129; three quarters grooved, 129

McDonald Corrugated, 78. 79

McGregor, John C., 140, 145, 146

Medicine cylinders, 128

Mesa Redondo, 148, 162

Mesa Verde, 203

Metatc, 48; fragments, 20

Metates, 122-124, 126, 147: troughed, $116,118,122,123,147$; basin, 116,122 , 123,147 ; slab, 118,124 ; flat, 122

Microclimate, shift in, 226

Migrations, 224-227

Milling area, 20, 24, 25, 62

Milling stones, 62, 126

Mimbres Polychrome, bowl, female figures in yellow, 72

Mindeleff, Victor, 65, 66, 67

Mineer, Mrs. Leola, 5

Mineral Creek, 40, 217

Mineral Creek Pueblo, 60, 62, 63, 67, 162, $182,186,187,190,218$

Miniature jar, 58, 59, 71, 142, 214

Modified Basket Maker period, 124

Mogollon, agriculture, 191; architectural development, 221; area, 126, 130, 132; culture, $129,144,163,166,167,191$; Great Kivas, 65, 67, 68, 214; ideas, 166; migration, 160; pithouses, 65; pithouse kivas, 66; San Simon Branch, 120; techniques, 164; tradition, 64, 161; Tularosa, 214; villages, 218,219

Mogollon Rim, 169, 204

Mormon tea, 174

Morris, E. H., 60, 66, 144, 145

Mortars, 26, 33, 37, 40, 45, 124-125, 126

Movement of peoples, 227; see also $\mathrm{Mi}$ grations

Murivinmana, 67

Muriyinwu, 67

Naegle, Mr. and Mrs. Cecil, 6

Nantack Phase, 64

Nantack Village, Great Kiva, 60; Ruin B, $62,65,124$

National Science Foundation, 3, 5, 148 , 210,222

Navahos, 227

Navaho sand paintings, 72

Nesbitt, Paul H., 73, 132, 144

Niches, 46, 48, 54, 55, 64, 66, 67, 68; -cache, 67,73 ; "Kachina," 51 ; pliallic. 67: wall, 68

Novak, Lillian, 6

Nuarez, Genaro, 4

Nuclear families, 210

Oak, 177, 179

Olson, Alan P., 63, 64, 65, 66, 67, 212

Open house, 5
Opler, Morris E., 205

Orientation, eastward, 67, 68, 213; northsouth, 66, 67; primary, of Great Kiva, $64,65,68$; village, 220

Ornaments, 140

Padilla, Gilbert, 4

Paint grinding, 126; red, 51

Palynological laboratory, 168; inquiry, 225

Parklands, 173, 188, 189, 191, 195, 196, 200

Parsons, Elsie C., 67, 69, 71, 72

Passageway, 37

Peckham, Stewart, 62

Pecos, 146; Classification, 150

Penasco Phase, 122

Pendant, bone, 143, effigy, 140; bone and shell, 140; clam shell, 143; limestone, white, 143; turquoise, 143

Pendants, tinkler, conus, 147; unfinished, 140,143

Penrod, Mr. and Mrs. Floyd, 6

Penrod, Kenneth, 4, 6

Penrod, Mr. and Mrs. Leonare!, 6

Pepper, George H., 144

Percussion flaking, 126; chipping, 130, 136

Perry, Martha, 4

Pestles, 120-122, 126; cylindrical, 120 ; multiface, 120, 122; pear-shaped, 120, 122

Phipps, Mr. and MIrs. Claude, 6

Pictographs, 54, 69, 73

Pigments, grinding of, 126

Pigweeds, 174, 200

Pillars, 65

Pinedale, 145

Pinedale Black-on-White, 151

Pinedale Polychrome, 151

Pine Lawn, area, 164, 169, 180, 181, 185 , 186, 188, 191, 196, 227; Phase, 61, 122, 132; Valley, 161

Pinnawa series, 214

Pinto Basin, 156

Pinto Point, 155

Pinus, 173, 177-180; edulis, 173; ponderosa, 173

Pinyon, 173

Pithouses, 212; D-shaped, 26

Pithouse village sites, 178,200

Pits, $19,22,29,34,54,58$; resonator. 67 ; storage, 25, 26; sub-floor, 180

Plaiting, 140

Plants, common names, 174

Plaster, 26, 33, 45, 54, 62; adobe, 46, 62

Platcau, 203, 204

Platform, 51, 66

Platyopuntia, 174

Plaza, 43, 65, 218, 220

Point of Pines, 60, 61, 63, 64, 122, 124 , $125,132,139,204,206$; people, 227; region, 224

Polishing stones, 126 
Pollen analysis, 3, 168-208, 221, 225; program, 222; chronology, 169, 189, 190, 198; extraction technique of, 207-208; types, common names, 173, frequencies of, 227

Population, 200, 201.215, 216, 218, 221; clensity of, 224, 225; increase, 204; movements, Cohonina, 203, 227

Postholes, 20, 22, 25, 29, 33, 34, 37, 59, 67; donble, 59

Posts, 37, 65; recessed, 54, 59

Pot covers, 125, 127, 147

Pot rest stone, 48

Pot rests, 126, 147

Pottery, black-on-white, 115; hachured, 115; lack of, 116

Pottery-making. tools, 126

lottery, design elements, 75, changes in, 76, 77; "horizon styles," 75, 77; "intrusive," 76; methodological considerations (statistics), 88; miniature jar, 58, 59; painted, statistical analysis of, 75 ; petrographic analysis, 76; relationships between types, 107, 108; relative positions of samples, 90; seriation, 80, 87, 88, 93, 94; size of sample, 89; stylistic changes, 77 , drift in, 77; temporal sequence of sites, 88, 89; "trade," 76; trade, Anasazi, 201; trends in design elements, 109, in painted pottery types, 97: whole (or restorable), 78; see also Design Elements

Pottery types, Abajo Red-on-Orange, 105; Alma Plain, 150, 159; Black Mesa Black-on-White, 105, 106; brown indented colrugated, 79; Four Mile Polychrome, 73, 214; Heshota-uthla Polychrome, 80, 214; Houck Polychrome, 80, 213; Kana-a Black-on-White, 105, 106: Kiatuthlanna Black-on-White, 102, 105-109, 150, 212, 213, 223; Kwakina Polychrome, 85, 214; Ia Plata Black-on-Orange, 105; Lino Gray. 150 , 159; McDonald Corrugated, 78, 79; Mimbres Polychrome, 72; Pinedale Black-on-TWhite, 151; Pinedale Polychrome, 151; Red Mesa Black-onWhite, 78, 102, 105-110, 150, 212, 223, later, 110 ; Reserve Black-on-White, $102,105,107,150,212,213,219$; St. Johns Polychrome, 80, 151, 213, 214 ; Snowflake Black-on-White, 5, 75, 78, $102,105-110,150,212,213,219,223$, design elements, 110 , lineage of, 75, 224; Sosi Black-on-White, 105; Tularosa Black-on-White, 102, 103, 105, $107,151,212,213,214,219$; Tularosa White-on-Red, 213; Tusayan White Ware, 163; White Mound Black-onWhite, 102, 150; Wingate Black-onRed, 103, 150, 213, 214, 219; Woodruff Smudged, 78
Powamu, ancestral to, 68

Prayer sticks, 134

Pre-ceramic site, 211

Precipitation pattern, 193; winter, 225, 226; see also Rains

Procedure, field, 151; statistical pottery analysis, basic, 90

Projectile points, 130-132, 133, 147, 158; cliagonal notehed, 132; barbed, 147; triangular, small, 147

Promontory Site, 61, 169, 179, 180, 181, $185,186,188-191,206$

Proto-kachina, 71, 73, 74

Pueblo, dwelling units, 200, 201; early example of, 218; farmers, 227; sites, 181-185; true, 220

Pueblo I sites, 60; III sites, 60, 119, 174; IV $V^{\gamma}$ sites, 119

Pueblo Bonito, 68, 144, 146

Pueblo Indians, contemporary, 72

Punches, 135, 138

Quemado area, 68

Quercus, 173, 177, 179

Quiburi, 144

Rafters, 20; see also Roof supports

Rain farmers, 201, 203

Rains, summer, 193, 196, 197, 198, 201205, 226; winter, 193, 197, 198, 201; see also Precipitation

Rands, Robert L., 77

Red Mesa Black-on-White, 78, 102, 105109, 150, 212, 223; design elements, 109, 110; later, 110

Red paint, grinding, 51

Reed, Erik K., 201, 203

Reiter, Paul, 66, 67, 68

Relative chronological order of sites and rooms, 89

Reserve area, 51, 60, 61, 63, 64, 119, 120, $122,124,125,132,138,139,227$

Reserve Black-on-White, 102, 105, 107, $150,212,213,219$

Reserve Phase, 61; late, 50

Reserve-Tularosa series, pottery, 163

Resonators, 67

Rhoton site, intra-site seriation, 94; pottery of, 77,102

Rhoton, Verl, 5

Richey, Leigh, 5

Riley, Carroll L., 205

Rim Valley Pueblo, 3, 40-53, 62, 63, 117 $122,124-131,136,138-141,143,146$, $182,190,213,218,221$; intra-site seriation, 98; pottery of, 77, 78, 80, 102, 103, 106, 108, 109; Room C, 51, 220; settlement pattern, 218; summary, 213; totals of sherds, 84 ; trends in painted pottery types, 97

Rinaldo, Mrs. John B., 4, 106 
Ring, bone and shell, 144, 147; fragment, 143; material, 141; slab, 47, 57, 214

Rio Grande area, 119, 134

Ritual circuit, directional colors, 69

Robbins, IVilfred WVilliam, 174

Roberts, F. H. H., Jr., 60, 66, 67, 124, 129, 132, 201

Robinson, W. S., 87, 107

Robinson-Brainerd seriation technique, 87; criticism of, 87; choice of, 87 ; $\mathrm{co}$ efficient of similarity, 90 ; basic procedurc, 90

Romane, Pat, 4

Roof, 20, 22, 25, 29, 37, 40, 59; crib-like, 61: supports, 64, 65, 68; timber, 130

Room shape, 62

Rubbing stones, 119-120, 147

Rubble; see Masonry

Sackheim, Judd, 6

Sacred stone image; see Image

St. Johns, 19, 148, 224

St. Johns Polychrome, 80, 151, 213, 214

St. Johns-Salt Lake Highway, 26

Salix, 173, 177

San Cayetano, 144, 145

San Francisco levels, Tularosa Cave, 140

San Francisco Phase, 138

San Francisco River, 181, 186

San José, 156

San Josć Point, 155

San Simon Branch, 120; Village, 144

Sapir, Edward, 108

Saul, John, 4

Sawmill Site, 60, 65, 68

Saws, 135, 136-139; smooth, 138

Sayles, E. B., 61, 65, 116, 120, 122, 144

Schroeder, A. H., 204, 205

Schulman, Edmond, 206

Schwartz, Douglas IV., 203, 204

Scraper-planes, 136

Scrapers, 131, 132, 135, 136, 147, 158161; gourd, 126; large, 136; oval biface, 130; small, 136,137

Secular structures, 60; use, Room C, Rim Valley, 51, 220

Sedge family, 174, 195

Sediment samples, 170

Seriation, pottery, 80 ; inter-site, 93 ; intrasite, 94; tcchnique, 87

Settlement patterns, 69, 164, 200, 215-222

Shell, beads, 140; bracelets, 140 ; pendant (with bone), 140; ring, 140, 147

Shells, Pacific Coast, 115

Sheiter, light brush, 61

Shipaulovi, kivas, 65

Show Low, 29, 148, 224, 227; arca, 152

Show Low Black-on-Red, 150

Show Low-Silver Crcck clrainage, 224

Sichomovi, 67

Sills, 46
Silver Creek, 166; see also Show LowSilver Creek

Similarity; see Coefficients of Similarity

Sinagua, 204, 227

Sipapu, 51, 220

Site 481, 68; Site LS-4, 180, 187, 191; Site LS-24, 185; Site LS-28, 181, 190, 191: Site I.S-34, 185, 187, 190; Site LS-50, 180; Site 30, 62, 169, 180, 186, 188, 191, 206; Site 30-31 arroyo, 186; Site 31,61

Site locations, 152, 164, 200, 201, 216 , 218, 220; favorite, 224; pre-pottery, 224

Slabs, 54, 55, 59, 65, 122; image, 58; perforated, 67 ; ring, 47, 57, 67; sandstone, $45,53,213$; stone, $51,57,62,63,220$; worked, 67

Smiley, Terah L., 4, 60, 168

Smith, Watson, 66, 68, 71, 72, 73, 105 , 146

Snaketown, 140, 144, 145

Snowflake, 148, 162; region, 163, 227

Snowflake Black-on-IVhite, 5, 75, 78, 102, $105-110,150,212,213,219,223$; design elements, 110 ; lineage of, 75,224

Snowflake-Mesa Redondo, 152, 221

Social history, 211; organization, 216 , $217,218,220,222$

Soil zones, 197

Sosi Black-on-White, 105

Southern Illinois University, 168

South Leggett Site, 181

Spicer, Edward H., 140, 146

Spindles, 134

Spindle whorls, 139, 140, 142, 147; of Mexican derivation, 140

Springerville, 19, 40, 53, 60, 148, 224, 227

Squash, 174; kachina, 72; pollen of, 221

Statistics, Jexico-, 205

Stephen, Alexander M., 67

Stcppe zone, 192, 194, 202-205

Stevenson, Matilda C., 71

Stcwart, G. R., 203

Stone, bowls, 125; discs, 125 (see Pot covcrs); tools at pithouse village, 210,211

Storagc pits(?), 25, 26, 62, 215; techniques, 164

Stradling, Frank, 4

Strassburger, Roland, 4

Sudatories, 68

Summer rains, 193, 196-198, 201, 203, $204,205,226$

Sunflower family, 174

Sunset Crater, 204

Supra-village organization, 222

SU Site, 116, 121, 146, 169, 179, 185, 188

Table Rock Pueblo, 60, 63, 67, 124, 126, $145,169,184,185,190$

'Talatumsi ceremony, 72

'laylor, Dr. Walter W., 168 
Technique, of pollen extraction, 207-208; of sample collection, 206

'Te'cui, 146

'Temporal sequence of sites (pottery), 88

Terracing systems, 204

Thode, Earl, 4

Thode Site, 3, 38, 39, 40, 61, 62, 118, 120, $125,130,136,139,140,141,145,219$; pottery of, $77,78,80,102$; settlement pattern, 217; summary, 212-213; totals of sherels, 83

Thomas, 'Tully H., 156, 157

Thompson, Raymond H., 212

Three Circle Plase, 64

Three Pines Pueblo, 62, 124

lihkuyi, 67

lihkuyiki, Childbirth water house, 67

linkler, conical, 145, 147

liticv, Mischa, 71,73

Trade, evidence of, 115; pottery, Anasazi, 201

Trait unit intrusions, 115

'Trewartha, G. T., 192

"Triangle," 151, 166

Tseh Tso, 126

Tularosa Black-on-White, 102, 103, 105, $107,151,212,213,214,219$

Tularosa Cave, 140, 155

Tularosa Mogollon, 214

Tularosa Phase, 63, 132; early, 50; end of, 51; tradition of the Mogollon, 68

Tularosa White-on-Red, 213

Tumbleweed Canyon Site, 3, 19-26, 61, $116,117,118,121,122,131,132,134$, $136,137,157,164,165,166,178,188$, 190, 191; settlement pattern, 215-216; summary, 210-211

Turkey Foot Ridge, 65, 180, 185

Tusayan White Ware, 163

Tuwabontumsi, sand altar woman, 67

Tuwabontumsiki, phallic niche-cache, 67

Tuwapongtumsi (a cult deity), 73

'Tuzigoot Ruin, 140, 146

rwin Butte site, 124

Typha, 173, 177, 183, 184, 185, 190, 195, 196

Underworld; see Cult deity

Urbanization, 219

Vaults, 48, 51, 56, 57, 60, 64, 66, 67; footdruin type, 51, 220; masonry-lined, 115, 214 ; north (ycllow pigment), 71 ; south(rn, 67, 68; use of, 68 ; western, 67

Ventana Cave, 116, 156, 178

Ventilator, 36, 37, 46, 48, 49, 51, 59, 62, $63,66,220$
Verde Valley, 205

Vernon, 61; area, 5, 169, 178, 180, 186, 189, 191, 196; project, 6

V'estigial architecture, 219

Village control, 221

Villages, form of, 61,212

Vivian, Gordon, 66, 67, 68

Voth, H. R., 66, 69, 71

Waard, 1)r. H. de, 211

Walnut, 177, 182

Walls, 19, 20, 23, 26, 32, 37, 40, 43, 46, 53, 217; defense systems, 210; extensions of jacal construction, 62

Warfare and hunting, tools, 130-139

Wasley, William W., 212

Water, standing, 195, 196, 202; tables, $197,198,200,201,204,226$

Weaving tools, 139-140

Weed, tolerated, 174

Welch, Dr. Walter B., 168

Wells, John, 4

Wendorf, Fred, 63, 124, 125, 132, 139, $144,145,146,157,158,212$

Western Pucblo kivas; sce Kivas

Wetherill Mesa, 174

Wet I eggett arroyo, 169, 177

Wet Leggett Cochise, dwelling area, 61

Wet Leggett site, 116

Wheat, Joe Ben, 64, 121, 122, 132, 139

White Mfound Black-on-White, 102, 150

White Mountains, 148, 152, 224

Whiting, A. F., 174

Whiting, Mr. and Mrs. Eben, 6

Wikwalobi kiva, 67

Willey, Gordon, 77

IVillis, Mr. and Mrs. Ira, 5

Willis, Kelley, 5

Willow, 177

Wilson, Ozie, 5

Wilson, Mr. and Mrs. Richard, 5

Wiltbank, Pacer, 4

Wingate Black-on-Red, 103, 150, 213, 214,219

Wodehouse, R. D., 171

Woodbury, Richard, 119, 146, 204, 206

Woodland, Bertram J., 6

Woodruff Smudged, 78

Wuwutcim ceremony, 72

Wuya, clan, 71

Wyman, Leland C., 72

Zea, 173, 177, 179-184, 190, 191, 196

Zoogamous pollen type, 174

Zuni area, 145; culture, 167; Indians, 227; kiva, 66; similarities, image, 69, 71; towns, 22, pre-Spanish, 218 




Publication 954 


UNIVEASTTY OF ILLINOIS-UABANA 